

\title{
digital humanities austria 2018 empowering researchers
}

ISBN: 978-3-7001-8668-7

DOI: https://doi.org/10.1553/dha-proceedings2018

\author{
List of editors: \\ Marlene Ernst \\ Peter Hinkelmanns \\ Lina Maria Zangerl \\ Katharina Zeppezauer-Wachauer
}

With the collaboration of Verena Höller.

Peer reviewed.

November 29-December 01, 2018, University of Salzburg, Austria The texts of the abstracts are reproduced as submitted - including formatting of accentuations, titles etc. The opinions and views expressed are those of the authors and have not been verified by the meeting organisers, who accept no responsibility for the statements made or the accuracy of the data presented. 


\section{INHALT}

6 EMPOWERING RESEARCHERS

Einleitende Worte zu den Proceedings der digital humanities austria Conference 2018

Marlene Ernst, Peter Hinkelmanns, Lina Maria Zangerl \&

Katharina Zeppezauer-Wachauer

10 DIGITAL PRIMARY SOURCES - RAUM FÜR PRIMÄR DIGITALE ANGELEGENHEITEN

Von Definitionen der Digital Humanities über das digitale ÖKosystem als Primärquelle bis Zur ERHALTUNG Von Digitalen QuelLen

Gernot Hausar

16 MUSEUMSAPPS

Individualität Versus Plattformstrategie

Wolfgang Dorner \& Mariann Juha

21 DIGITISING PATTERNS OF POWER

An Interactive Map-Based Application to Represent Historical Landscapes

Markus Breier, Adam Mertel, Karel Kriz \& Alexander Pucher

29 THE SHAPE OF THE EARTH

A Round World as Common Worldview within the General Public in the Roman Imperial Period?

Sara Wanek

34 DIAL. DAS DIGITALE ILSE AICHINGER LITERATURVERZEICHNIS

Methoden, Quellen und eine erste Auswertung des Literaturverzeichnisses

Andreas Dittrich

40 DIE DIGITALE MOZART-EDITION (DME)

INTERdisZiplinäre ProjeKTE UNTER EINEM DACH

Mirijam Beier \& Till Reininghaus

46 SAVING DIGITAL HUMANITIES

„LANGzeitarchivierung by Design“

Richard Hörmann \& Daniel Schlager

54 FEATURE ANALYSIS OF PAINTINGS USING COLOR INFORMATION OF THE IMAGE

Ryuhei Yukimura, Hao Sun \& Mingzhe Jin 
62 TRAVELOGUES: FREMDWAHRNEHMUNGEN IN REISEBERICHTEN 1500-1876

EINE COMPUTERGESTÜtZTE ANALYSE

Doris Gruber, Martin Krickl, Lijun Lyu, Jan Rörden \& Arno Strohmeyer

67 BIBLIOTHEKSDATEN IN DH-FORSCHUNGSPROJEKTEN

EINDRÜCKE DES ROUND TABLES BEI DER DHA2018

Markus Bürscher \& Lina Maria Zangerl

70 THE ROLE OF ANALOG PUBLICATIONS IN A DIGITAL WORLD

The Archaeological Case Study CELSUS

Nicole M. High-Steskal

74 ÜBERLEGUNGEN ZUR DIGITALEN ARCHÄOLOGIE UND ZU IHREM VERHÄLTNIS ZU DEN DIGITALEN GEISTESWISSENSCHAFTEN

Dominik Hagmann

79 DEVELOPMENT OF A CROSS-PROJECT CITIZEN SCIENCE PLATFORM FOR THE HUMANITIES

Hendrikje Carius, Christiane Kuller, Patrick Rössler \& René Smolarski

83 DIE EDITION DER MINISTERRATSPROTOKOLLE 1848-1918 DIGITAL

Workflows, MöGLICHKEITEN, GRENZEN

Stephan Kurz, Wladimir Fischer-Nebmaier, Dario Kampkaspar, Richard Lein, Anatol Schmied-Kowarzik

91 COMMUNITY INVOLVEMENT FOR TRANSCRIBING HISTORICAL CORRESPONDENCES OF SOUTH TYROLEAN INTEREST

A DI-ÖSS USE CASE

Alexander König, Verena Lyding, Elisa Gorgaini, Georg Grote \& Monica Pretti

95 SOZIALE NETZWERKANALYSE UND ERZÄHLSCHEMATA

EINE EXPLoRATIVE Vorstudie

Friedrich Michael Dimpel

112 HISTORIOPLUS

Fünf Jahre Studieren und digitales Publizieren in Salzburg

Elias Knapp, Hester Margreiter \& Arno Strohmeyer

117 MARKETING TRIFFT FORSCHUNG

ZIELGRUPPENORIENTIERTE DigitalisIERUNG WISSENSCHAFticher INHALTE

Johannes Ebner, Peter Färberböck \& Stefan Schwaiger 
123 DER NACHLASS ALS NETZWERK

Zur Entwicklung einer Nachlass-Ontologie am Beispiel des Projekts

„Stefan ZWeig digital“

Christopher Pollin \& Lina Maria Zangerl

128 IDENTITÄT UND NORMDATEN

Joseph Wang \& Ulrich Lobis

134 ZWEI ÜBERLEGUNGEN ZUR KONZEPTION EINER LINKED-DATA-ONTOLOGIE FÜR DIE LITERATURWISSENSCHAFTEN

Bernhard Oberreither

140 FORSCHUNG ÖFFNEN

Möglichkeiten, Potentiale und Grenzen von Open Science am Beispiel der offenen Datenbank „HANDKe: In ZUnGen“

Vanessa Hannesschläger

145 SCHREIBEN, WIE GODARD FILME MACHT

Präliminarien eines intermedialen EditionsprojektS

Wolfgang Straub

150 ANNOTATIONEN UND IHRE INFRASTRUKTURELLEN PRÄMISSEN

Philipp Hegel, Germaine Götzelmann, Michael Krewet \& Danah Tonne

154 KULINARISCHE TRANSFERS AUS ANDEREN REGIONEN UND KULTURRÄUMEN: DER NIEDERSCHLAG IN WESTDEUTSCHEN UND ÖSTERREICHISCHEN KOCHBÜCHERN 1950-1980

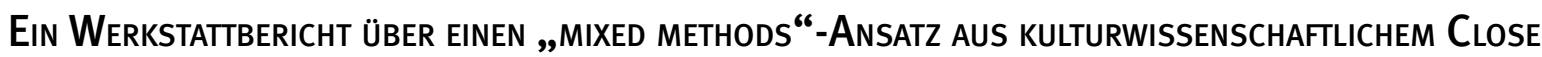
Reading Und digitalen Methoden

Christoph Bläsi \& Edith Blaschitz

160 KEYWORD INDEX 


\title{
Empowering Researchers
}

\author{
Einleitende Worte zu den Proceedings der \\ Digital humanities AUStRia Conference 2018
}

Marlene Ernst [1], Peter Hinkelmanns [2], Lina Maria Zangerl [3] \& Katharina Zeppezauer-
Wachauer [2]

[1] Universität Salzburg - Science \& Technology Hub, Wissens- und Technologietransfer \& Citizen Science

marlene.ernst@sbg.ac.at, https://www.uni-salzburg.at/forschung/citizen_science

[2] Universität Salzburg - Mittelhochdeutsche Begriffsdatenbank (MHDBDB)

peter.hinkelmanns@sbg.ac.at, https://orcid.org/0000-0001-8618-0185

https://uni-salzburg.elsevierpure.com/de/persons/peter-hinkelmanns-4

katharina.wachauer@sbg.ac.at, https://orcid.org/0000-0001-9310-9029

https://uni-salzburg.elsevierpure.com/de/persons/katharina-zeppezauer-wachauer-4

[3] Universität Salzburg - Literaturarchiv Salzburg

linamaria.zangerl@sbg.ac.at, https://orcid.org/0000-0001-9709-3669

https://uni-salzburg.at/literaturarchiv

Der Forschungsbereich Digital Humanities (DH) in Österreich durfte in den letzten Jahren beachtlichen Zuspruch erfahren. Seit 2014 veranstaltet das Netzwerk Digital Humanities Austria (dha) zusammen mit Partnern aus dem DH-Bereich eine jährliche Konferenz, bei der Forschende Erfahrungen austauschen, neue Methoden diskutieren und sich vernetzen können. dha ist ein Verbund mehrerer institutioneller Partner, deren Ziel es ist, die DH in Österreich zu fördern und den Einsatz digitaler Methoden in den Kultur- und Geisteswissenschaften zu unterstützen sowie integraler Bestandteil des österreichischen Engagements in den europäischen Forschungsinfrastrukturkonsortien CLARIN-ERIC und DARIAH-EU (CLARIAH-AT für Österreich). Die 5. Tagung digital humanities austria (dha2018) fand vom 29. November bis 1. Dezember 2018 an der Paris Lodron Universität Salzburg, einem Mitglied des CLARIAH-AT-Konsortiums, statt. Organisiert und unterstützt wurde die Konferenz von der Kontaktstelle Citizen Science, der Mittelhochdeutschen Begriffsdatenbank (MHDBDB), dem Literaturarchiv Salzburg sowie der DH-Plattform dhPLUS der Universität Salzburg.

Digital Humanities modellieren ein neues Setting für die Forschenden: Sie verändern die Wissensumwelt, schaffen zeitgemäße Zugänge, unterstützen Forschende wie Rezipierende, bieten neue Wege für etablierte Methoden und ermöglichen gesellschaftliche Teilhabe am Forschungsdiskurs. Expert*innenwissen wird dezentriert und demokratisiert. Gerade die digitalen Methoden in den Geisteswissenschaften sind dafür prädestiniert, einander paritätisch auf Augenhöhe zu begegnen, sowohl innerhalb akademischer Strukturen als auch außerhalb der insitutionalisierten Wissenschaftspraxis. In diesem Sinne stand die dha2018 unter dem Motto Empowering Researchers und umfasste Workshops, Podiumsdiskussionen und Präsentationen aus verschiedenen Forschungsbereichen, die sich der zentralen Frage widmeten, wie in den digitalen Geisteswissenschaften Gemeinsamkeiten, Interdisziplinarität 
sowie Kollaboration gestärkt werden können. Diese Aspekte schlugen sich auch in den teils unkonventionellen Formaten (etwa dem erfreulich gut besuchten Speed-Geeking) und den thematischen Schwerpunkten der Tagung nieder:

\section{Geisteswissenschaft stärken: Aufbruch in ein neues Zeitalter der Digitalisierung und Information}

Die DH erfinden die Geisteswissenschaften nicht neu, sondern erweitern die Möglichkeiten der etablierten Forschung. Durch den Einsatz von Datenverarbeitung und die Unterstützung automatisierter Verfahren ermöglichen sie neue Fragestellungen in den Geisteswissenschaften, die ohne diese Methoden nicht denkbar wären. DH-Zentren begleiten Projektprozesse der universitären Forschung von der Antragsphase bis zur Langzeitsicherung. Unter diesem Thema waren Beiträger*innen aufgefordert, sich mit Fragen nach der Beförderung des Wissenstransfers in den $\mathrm{DH}$ und den damit einhergehenden Herausforderungen zu beschäftigen.

\section{Gemeinsamkeiten stärken: Datennormierung als Chance}

Das Semantic Web, Linked Open Data und Ontologien haben großes Potential, Forschungsdaten auf internationaler Ebene zu vernetzen. Standards und De-facto-Standards der DH und des Bibliothekswesens wie TEI, CIDOC CRM oder MARC21 haben eine große Verbreitung, jedoch überschneiden sich oftmals die Anwendungsbereiche und es gibt rasche Veränderungen. Gleiches gilt für normierte Entitäten etwa von WikiData, GND oder VIAF. Aus dieser Vielfalt der Standards und Datenmodelle ergeben sich für die Interpretation und Nachnutzung in der Praxis neue strategische sowie methodologische Aufgaben. Im Rahmen der Konferenz wurde das Thema der Datennormierung aus unterschiedlichen theoretischen und projektspezifischen Perspektiven beleuchtet.

\section{Interdisziplinarität stärken: Vernetzte Forschung, vernetzte Forschende}

Trotz des Rufs nach Interdisziplinarität ist die Umsetzung von entsprechenden Projekten noch immer schwierig, wie auch Forschungsausschreibungen der letzten Jahre belegen, die gerade die Zusammenarbeit über Fachdisziplinen hinweg fördern wollen. Können vernetzte Forschungsdaten dazu führen, dass auch die Forschenden selbst besser interdisziplinär zusammenarbeiten? Die heterogenen Beiträge nahmen insbesondere Komplexität, Non-Linearität und Hybridität in den $\mathrm{DH}$ in den Fokus und zeigten, wie konzeptuelle Reformen einer durch Fachidentitäten tradierten Wissenschaftspraxis dazu beitragen können, die $\mathrm{DH}$ in ihrer vereinenden Tätigkeit zu unterstützen.

\section{Kollaboration stärken: Forschung öffnen}

Wissenschaftliche Forschung bleibt der Gesellschaft häufig hinter Bibliotheksmauern oder Paywalls verborgen. Durch die Visualisierung und Verfügbarmachung von Forschungsergebnissen haben die DH das Potential, eine Brücke zwischen Gesellschaft und Forschung zu schlagen. Dass sich die Wissenschaften öffnen, zeigt sich auch an anderen Initiativen, die oftmals parallel zu und in engem Kontakt mit DH entstanden sind: Citizen Science, Social Media, Co-Creation, Crowdsourcing, Open Innovation und Open Science sind die neuen Schlagworte der Forschungsvermittlung. Wie das Vorhaben der Öffnung gelingt, konnte auf der Konferenz an eindrucksvollen Beispielen gezeigt werden. 
Besonders hervorheben möchten wir zudem zwei Veranstaltungspunkte, die auch ohne Registrierung von der breiten Öffentlichkeit besucht werden konnten: die abendliche Keynote, gehalten von Dr. Sven Fund mit dem verheißungsvollen Titel Open Access in den Geistes- und Sozialwissenschaften: Was jetzt zu tun ist und die Lunchlecture im traditionsreichen Salzburger Mozartkino, auf der die Praxismethode Gamification im Rahmen eines Panels mit Expert*innen aus Forschung und Wirtschaft diskutiert wurde.

Sven Fund, seit über 15 Jahren im Medienbereich tätig und bekannt als ehemaliger Geschäftsführer der Wissenschaftsverlage De Gruyter und Peter Lang, derzeit an der Spitze des Berliner Kunstbuchverlags Hatje Cantz, widmet sich inzwischen verstärkt einer zeitgemäßen, offenen Forschungs- und Medienwelt: Nach Stationen in der zentralen Unternehmensberatung von Bertelsmann, verschiedenen Rollen innerhalb von Springer Science+Business Media, gründete er 2015 das Verlagsberatungsunternehmen fullstopp, ${ }^{1}$ Society for Digitality, das sich auf wissenschaftliche Publikationen sowie Institutionen im Bildungsbereich spezialisiert hat. Außerdem ist er Geschäftsführer der Plattform Knowledge Unlatched, ${ }^{2}$ die weltweit freien Zugang zu wissenschaftlichen Inhalten zum Ziel hat und eine zentrale Anlaufstelle darstellt, um Open Access-Modelle führender Verlage und neue OA-Initiativen zu unterstützen. Das Schlagwort Open Access ist seit Jahren in aller Munde und wird allerorts kontrovers diskutiert, wobei es jedoch oftmals beim philosophischen Theoretisieren bleibt. Fund begegnete diesem vertrauten Sermon mit erfrischend klaren Worten und widmete den Abend konkreten Fakten, indem er praxisnahe Wege und Taten aufzeigte, die sich heute für Forscherinnen und Forscher ergeben. Mit dieser Kernbotschaft gelang es ihm, einen theoretischen Bogen über die Themenblöcke der dha2018 zu spannen, der sich bis zum allerletzten Programmpunkt aufrechterhalten ließ: dem Common Sense, dass digitales Arbeiten nicht ausschließlich einer privilegierten Gruppe zur Verfügung stehen sollte, sondern unendlich viele Möglichkeiten eröffnet, unterschiedlichste Fachdisziplinen neu zu denken sowie Wissenschaft breitenwirksam zu kommunizieren.

Von Anfang an war uns die Vermittlung der DH außerhalb einer engen Community ein großes Anliegen. Aufgrund dieses in jedem Sinne offenen Charakters hinsichtlich von Methoden, Anwendungsmöglichkeiten und Rezipierenden, den auch zahlreiche Beiträge in diversen Sessions zu Citizen-ScienceThematiken unterstreichen, ${ }^{3}$ wurde bereits in einem frühen Planungsstadium eine öffentlich zugängliche Lunchlecture mitkonzipiert. In diesem Sinne wurde für die Mittagspause am zweiten Konferenztag (Freitag, 30. November 2018) das Mozartkino Salzburg als Schauplatz für eine Diskussionsrunde gewählt. In gemütlicher Atmosphäre in einem der ältesten Kinos der Welt, sprach man über das populäre Thema Gamification. ${ }^{4}$ Relevante Aspekte, Chancen sowie Risiken von Spielen von und für Forschung und Lehre wurden mit vier Expert*innen beleuchtet. Das Publikum von ca. 100 Personen, bestehend aus Tagungsteilnehmer*innen wie auch Interessierten außerhalb des Konferenzkontextes, konnte dabei seine Fragen an Univ.-Prof. Dr. Lisa Gotto (Universität Wien, Professorin für Theorie des Films), Peter

http://fullstopp.com/ (23.07.2019).

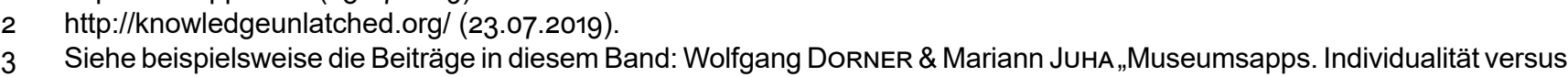
Plattformstrategie“; Hendrikje CARIUS, Christiane KULLER, Patrick RöSSLER \& René SMOLARSKI „Development of a Cross-Project Citizen Science Platform for the Humanities"; Alexander KöNIG, Verena LYDING, Elisa GoRGAINI, Georg GROTE \& Monica PRETTI „Community involvement for transcribing historical correspondences of South Tyrolean interest“; Vanessa HANNESSCHLÄGER „Forschung öffnen. Möglichkeiten, Potentiale und Grenzen von Open Science am Beispiel der offenen Datenbank ,Handke: in Zungen'“.

4 Unter Gamification versteht man den Einsatz von Game-Design-Elementen in Kontexten außerhalb klassischer Spiele, beispielsweise in Forschungskontexten. Zur detaillierten Definition siehe beispielsweise auch Sebastian DETERDING u. a., Gamification: Toward a definition, in: Proceedings of CHI 2011 Workshop Gamification: Using Game Design Elements 
Gietz (DAASI International), Dr. Harald Klinke, M.Sc. (LMU München, Digitale Kunstgeschichte) und Wilfried Reiter (Gamedesign/Webentwicklung, Mitbegründer der Anno-Reihe) richten.

Die dha2018 bot ein Forum für den Dialog der Fachdisziplinen und der über 160 Teilnehmer*innen aus beinahe zehn Nationen. Ein Großteil der Gäste stammte aus Österreich, jedoch durfte die Universität Salzburg auch Kolleg*innen aus Deutschland, Italien, Rumänien, Serbien, Spanien, Island und sogar aus Japan und den USA willkommen heißen. Der Austausch zwischen nationalen und internationalen Personen und Projekten trug wesentlich zum Erfolg der Konferenz bei. Dass es sich dabei nicht nur um Forschende aus dem engeren universitären Kontext handelte, sondern etwa auch zahlreiche Teilnehmer*innen aus dem tertiären institutionellen Forschungsumfeld vertreten waren, bereicherte die interdisziplinäre Veranstaltung ungemein: Beiträge kamen von Forscher*innen der Universität Wien, der Österreichischen Akademie der Wissenschaften, der Universität für Bodenkultur Wien, der Universität Graz, der Universität Innsbruck, der Katholischen Privat-Universität Linz, der Donau-Universität Krems, der Universität Salzburg, dem Mozarteum Salzburg, der Fachhochschule Salzburg, der GoetheUniversität Frankfurt am Main, der Johannes Gutenberg-Universität Mainz, der Friedrich-AlexanderUniversität Erlangen-Nürnberg, der Technischen Universität Darmstadt, dem Deutschen Literaturarchiv Marbach, der Universität Passau, der Universität Bamberg, der Technischen Hochschule Deggendorf, der Universität Göttingen, der Freien Universität Berlin, der Ludwig-Maximilians-Universität München, der Technischen Universität München, der Universität Regensburg, der Leibniz Universität Hannover, der Universität Erfurt, der Háskóli Íslands (Island), der Università degli Studi di Napoli (Italien), der Doshisha University (Japan), dem Bowdoin College (USA) sowie von Vertreter*innen nationaler und internationaler Gedächtnisinsitutionen wie etwa diversen Bibliotheken und Bibliotheksverbünde (Österreichischer Bibliothekenverbund, Vereinigung Österreichischer Bibliothekarinnen und Bibliothekare, Österreichische Nationalbibliothek, Universitätsbibliothek Mozarteum, Forschungsbibliothek Gotha der Universität Erfurt, Wienbibliothek im Rathaus, Diözesan- und Universitätsbibliothek an der Katholischen Privat-Universität Linz), von Museen (Stadtmuseum Graz, Residenzgalerie Salzburg), Archiven (Österreichische Nationalbibliothek, Literaturarchiv Salzburg, Österreichische Mediathek), außerdem von weiteren Forschungsinstitutionen (Research and Technology Organisation AIT - Austrian Institute of Technology, Internationale Stiftung Mozarteum, Eurac Research Bozen, Knowledge Unlatched), Vertreter*innen der österreichischen Politik (Land Salzburg, Bundesministerium für Bildung, Wissenschaft und Forschung) sowie Teilnehmer*innen aus der Wirtschaft (DAASI International - Identity \& Access Management, Content Marketing Agentur marke [ text, DevPoint Gamedesign, fullstopp - Society for Digitality).

Dem Herausgeber*innenteam war es ein großes Anliegen, die auf der Konferenz gebotene Vielfalt auch in den Proceedings widergespiegelt zu wissen. Auf den kommenden Seiten finden sich 26 thematisch bunt gemischte Beiträge von Open-Access-Strategien bis hin zu Linked-Open-Data-Überlegungen. (Kunst-)Historische Fragestellungen werden ebenso behandelt wie sprachwissenschaftliche, archäologische oder geografische Themen. Überzeugen Sie sich selbst von der Bandbreite der aktuellen Forschung im Bereich DH in Österreich und darüber hinaus.

Viel Freude beim Lesen wünschen Ihnen

Marlene Ernst, Peter Hinkelmanns, Lina Maria Zangerl und Katharina Zeppezauer-Wachauer

in Non-Game Contexts. 6-9, online unter: http://hci.usask.ca/uploads/219-02-Deterding,-Khaled,-Nacke,-Dixon.pdf (23.07.209). 


\title{
Digital Primary Sources - Raum für primär digitale Angelegenheiten
}

Von Definitionen der Digital Humanities über das digitale Ökosystem als Primärquelle bis zur Erhaltung von digitalen Quellen

\section{Gernot Hausar}

Universität Wien, Institut für Geschichte gernot.hausar@gmail.com / gernot.hausar@univie.ac.at https://orcid.org/0000-0001-5784-988X

Keywords: Internet, Digital Sources, Digital Archaeology, Digital Ecosystem, Digital as Primary Source

\begin{abstract}
In scientific interaction of digital humanities scholars with the digital ecosystem the focus is more on the use of digital tools and less on the digital ecosystem as a primary source. This is made visible both by the range of definitions and the focus of scientific endeavors. This paper argues that the consequences of this might be the loss of valuable digital only sources and offers some possible approaches and „hacks" to mitigate the damage and facilitate scientific cooperation.
\end{abstract}

\section{Primär digitale Angelegenheiten ${ }^{1}$}

Primär digitale Quellen verändern gleich einem ,trojanischen Pferd' alle Abläufe geisteswissenschaftlicher Forschung nachhaltig. Ein Anzeichen für die sich stellenden Herausforderungen bietet beispielsweise die Suche nach gemeinsamen Definitionen des ,Digitalen', die in Summe mehr als seine einzelnen Komponenten darstellt und sich ,verlebendigt '. ${ }^{2}$ Denn während sich die Digital Humanities die digitalen Werkzeuge, Metadaten und Kommunikationstools bereits weitgehend zu eigen gemacht haben, steht eine gemeinsame Arbeitsdefinition des digitalen Ökosystems und des Digitalen als Raum, die mit den sich rasch ändernden Rahmenbedingungen mitwachsen kann, noch aus.

1 Basiert teilweise auf: Gernot HAUSAR, Weitere Überlegungen zu digitalen Geschichts- und Geisteswissenschaften, in: Julia Menzel, Hg., Digitalität - Theorien und Praktiken des Digitalen in den Geisteswissenschaften 2016, online unter: https://web.archive.org/web/20190409173203/https://digigeist.hypotheses.org/308 (09.04.2019).

Wolfgang SchmaLE, Digitale Geschichtswissenschaft, Köln / Wien 2010, 15 f. 
Einen Überblick über die Vielfalt an Definitionen gibt beispielsweise das Projekt „What is Digital Humanities?“3, welches Definitionen von Teilnehmer*innen des Projektes „Day of DH“4 2009-2014 gesammelt und dargestellt hat.

Dies macht es nötig, auch hier auf die gewählten Begriffe kurz einzugehen:

- „Digitales Ökosystem“ umfasst sowohl die zugrundeliegende physische Infrastruktur (z.B. Kabel, Eingabe- und Speichergeräte, Computerchips) als auch die darüber ausgetauschten bzw. lokal gespeicherten und verarbeiteten Daten.

- „Digitaler Raum“ beschreibt die im digitalen Ökosystem existenten Räume, die im Sinne von Henri Lefebvres ${ }^{5}$ Ansatz durch die Nutzer*innen „produziert“ werden.

- „Digital Humanities“ wiederum umfasst die Untersuchung aller menschlichen Aktivitäten aus Sicht der „Humanities“ (e.g. Geistes- \& Kultur-, Kunst- und Sozialwissenschaften) durch Wiederherstellen, Erhaltung \& Archivierung sowie Untersuchung digitaler Vorgänge und Kultur mit technischen Hilfsmitteln.

Der Begriff des Digitalen selbst umfasst hier sowohl das Ökosystem als auch die durch die Nutzer* innen geschaffenen Räume. Diese Räume ermöglichen es uns, in rein digitalen Umgebungen Artefakte zu erschaffen, die in vollem Umfang nur in dieser Umgebung Bestand haben können.

Dabei gibt es im Moment widersprüchliche Signale in Bezug auf die Einordnung von rein digitalen Artefakten und digitalen Räumen. Vereinzelte Entscheidungen von Gerichten in Asien legen nahe, dass diese Räume als eine Art virtuelles Staatsgebiet zu sehen wären. Diese entschieden, dass virtuelle Ausrüstungsgegenstände in einem Computerspiel strafrechtlich relevant gestohlen werden konnten. ${ }^{6}$

Diese virtuellen Ausrüstungsgegenstände können in vollem Umfang ausschließlich in einem virtuellen Raum genützt werden. Auch mit Brückentechnologien wie dem 3D-Druck ist es nicht möglich, diese adäquat als physische Artefakte zu reproduzieren. Dies legt nahe, dass rein digitale Artefakte optimaler Weise in einer digitalen Umgebung analysiert und erhalten werden sollten. Digitale Artefakte sind als Primärquellen aufgrund ihrer Eigenschaften anspruchsvoll, denn sie sind fast untrennbar mit den Räumen verbunden, in denen sie existieren. Darüber hinaus sind sie relativ volatil, da sich die Räume und die Artefakte rasch ändern und das Artefakt leicht verloren gehen kann. Dies haben beispielsweise die Datenfehler bei MySpace im Jahr 2019 auf schmerzhafte Weise demonstriert: Durch den Verlust eines Backups ${ }^{7}$ gingen fast die gesamten dort gespeicherten Musikdateien und Inhalte der Seite unwiederbringlich verloren, denn auch das Internet Archive konnte nur 500.000 der über 50.000.000 Musikstücke wiederherstellen. ${ }^{8}$

3 Jason Heppler, What Is Digital Humanities?, online unter: https://whatisdigitalhumanities.com/ (og.04.2019).

4 LiNHD, Day of DH, online unter: https://web.archive.org/web/2018082823030g/http://dayofdh2016.linhd.es/ (og.04.2019).

5 Siehe dazu beispielsweise: Henri LefebVRE, Die Produktion des Raums. The Production of Space, Leipzig 2018.

6 Eric Jou, Two Gamers Are Going To Jail For Stealing \$6,405 In Virtual Items, online unter: https://web.archive. org/web/2019040916o834/https://kotaku.com/two-gamers-are-going-to-jail-for-stealing-6-405-in-vir-1571780985 (09.04.2019).

7 Siehe dazu beispielsweise: Cory Doctorow, Myspace lost all the music its users uploaded between 2003 and 2015 , online unter: https://web.archive.org/web/20190409162907/https://boingboing.net/2019/03/17/facebook-is-next.html (09.04.2019).

8 Internet Archive, The Myspace Dragon Hoard (2008-2010), online unter: https://archive.org/details/myspace_dragon_ hoard_2010 (09.04.2019). 


\section{Die unendliche Vielfalt der digitalen Quellen}

Der oben beschriebene Verlust digitaler Artefakte bei MySpace ist nur ein Beispiel für die Herausforderungen bei der Arbeit mit digitalen Quellen und deren Erhaltung, denn derer gibt es viele:

Der schiere Umfang der neu entstehenden Quellen ist kaum vorstellbar. Mit Oktober 2018 sind etwa 4,5 Milliarden Menschen im Internet aktiv - etwa 3,4 Milliarden davon sind Nutzer*innen sozialer Medien. So wurden laut einer Zusammenstellung von Domo ${ }^{9}$ im Jahr 2018 in jeder einzelnen Minute 3,8 Millionen Suchanfragen von Google abgewickelt und die User senden 15 Millionen SMS, 46.000 Bilder via Instagram und 159 Millionen Emails (wobei etwa die Hälfte davon Spam ist). In jeder Minute fließen 3 Millionen GB an Daten über das Netz, 63 Websites werden gehackt und 2.500 MW werden zur Aufrechterhaltung der Infrastruktur verbraucht.

Neben dem reinen Umfang steigt auch die Komplexität der Erhaltung. Denn zur Erhaltung eines Artefaktes muss oft auch der digitale Raum miterhalten werden. Dies beinhaltet die Erhaltung und Reproduktion der zugrundeliegenden Soft- und Hardware. Darüber hinaus gibt es oft physische Beigaben (z.B. Handbücher) und verwandte digitale Quellen, die Metainformationen über das eigentliche Artefakt enthalten (z.B. Diskussionsforeneinträge, Podcasts, Videos) und mit ihm erhalten und untersucht werden sollten (siehe Abb. 1).

\section{Elemente digitaler Artefakte}

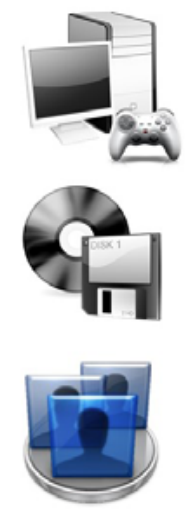

Zugrundeliegende Hardware \&

Connectivity

Software (Code, Kunst, Regeln, Beiwerke)

Erfahrungen, Erinnerungen, Austausch, Meta-Informationen

Abb. 1: Elemente digitaler Artefakte. Hausar 2019.

\section{Digital Humanities to the rescue?}

Die Digital Humanities bewegen sich in diesem Meer aus Code und Daten im Rahmen des digitalen Ökosystems. Sie leisten eine doppelte Arbeit indem sie den Brückenschlag zwischen den seit Jahrhunderten analog aufbewahrten Quellen und den originär digitalen Quellen versuchen, um eine Analyse primär analoger und digitaler Quellen in einheitlicher, digitaler Form zu gewährleisten. Dabei ist dieser Schritt auch eine Flucht nach vorne, da eine Analyse und Aufarbeitung ganz ohne digitale Werkzeuge fast nicht mehr möglich erscheint.

9 Domo, Data Never Sleeps 6.o, online unter: https://web.archive.org/web/20190409161923/ bzw. https://web-assets. domo.com/blog/wp-content/uploads/2018/o6/18-domo-data-never-sleeps-6.png (og.04.2019). 
Diese Werkzeuge sind auch bei der ,Übersetzung und weitergehenden Erschließung von (bereits aufgearbeiteten) analogen Quellen in digitale im Einsatz, die im Moment stark im Fokus der Forschung ist. Es ist daher auch nicht verwunderlich, dass digitale Elemente sich hauptsächlich über den Einsatz digitaler Werkzeuge in die Arbeit digitaler Humanisten ,einschreiben' und gerade in den Anfängen weniger Aufmerksamkeit auf den primär digitalen Quellen ruht.

Dies lässt sich auch gut über Begriffsvergleiche in Google Books ngram-viewer visualisieren. Während der Begriff „Humanities Computing“, der seit den frühen Siebzigerjahren in Gebrauch ist, stärker den Einsatz digitaler Rechenmaschinen referenziert, kommen in englischsprachigen Büchern seit den Neunzigerjahren vermehrt Begriffe auf, die weniger auf die Werkzeuge und mehr auf Eigenschaften bzw. Räume hinweisen. Der Gebrauch der Begriffe „Digital Archaeology“, „Digital History“ und seit dem Jahr 2000 auch vermehrt „Digital Humanities“ nimmt zu, während „Humanities Computing“ als Begriff weniger oft genutzt wird (siehe Abb. 2).

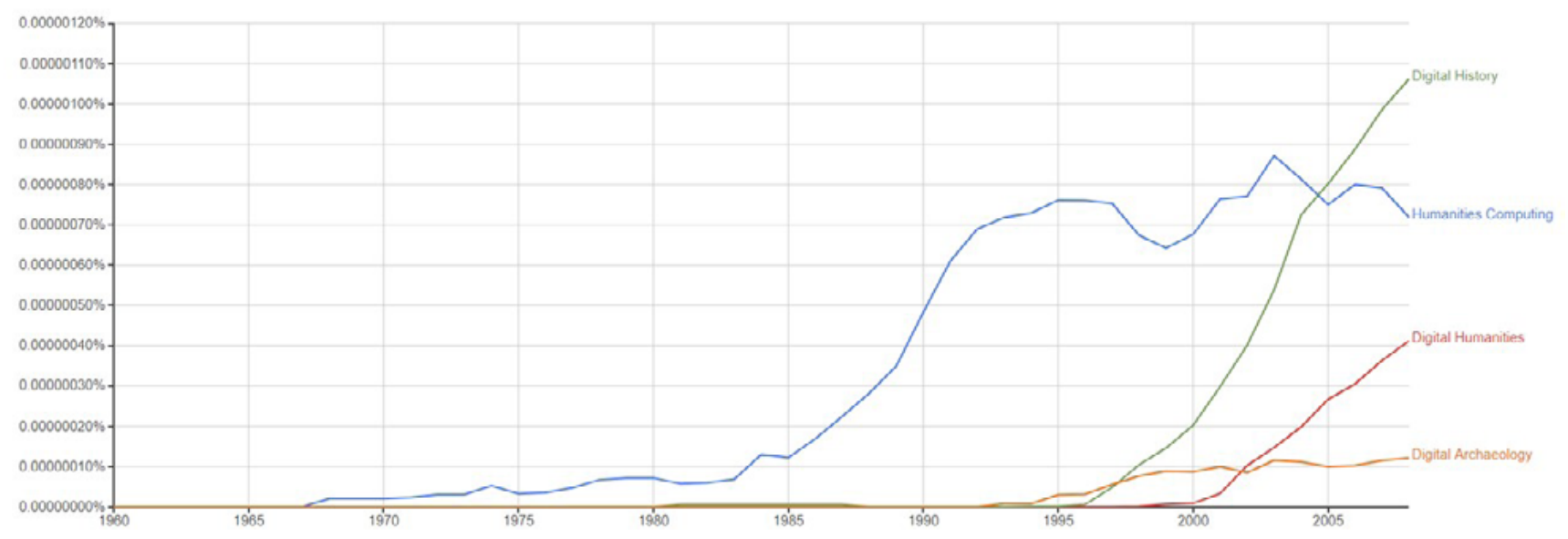

Abb. 2: Häufigkeit der Digital Humanities Begriffe im Vergleich. Google Books ngram Viewer (English Corpus, Smoothing 3). Hausar 2019.

\section{Die Kurzlebigkeit digitaler Quellen}

Der Erhalt primär digitaler Quellen ist manchmal sehr anspruchsvoll und gleicht dem Versuch, in warmem Klima langfristig Schneebälle in Kühlschränken aufzubewahren. Daher ist es auch durchaus verständlich, dass es kaum dezentrale und noch weniger zentrale umfassende Ansätze zur Archivierung der digitalen Primärquellen gibt, sodass wir von der Quellenlage her de facto seit den 1980er Jahren ein schwarzes Loch haben, welches durch "Citizen Scientists and Archivars“ (archive.org, Computermuseen, Hackerkollektive, Bootleg Archive etc.) sowie institutionelle oder regionale Initiativen (z.B. Phaedra an der Universität Wien bzw. ARCHE am Austrian Center für Digital Humanities der ÖAW) in geringem Umfang abgefedert wird.

Die Bemühungen von Citizen Scientists umfassen meist nur relativ statische Räume (z.B. Newsgroups, eMails, statische Websites, Single Player Games, Videos etc.), massive virtuelle Onlineumgebungen wie beispielsweise VR-Chatwelten (z.B. das immer noch existierende Second Life), Soziale Plattformen (Facebook, YouTube, Snapchat) oder Computerspiele wie MMRPGs (EVE Online, World of Warcraft etc.) sind hier noch gar nicht inkludiert. 
Schwierigkeiten bei der Sichtung bereiten dabei die sich ständig wandelnden Formen des digitalen Status quo bei dynamischen digitalen Quellen, die eine permanente Versionierung aller Artefakte erfordern würde und außerdem auch noch von der jeweiligen Hardware abhängt. Erst dann könnte man beispielsweise eine Begegnung mehrerer Avatare auf einer sozialen Plattform oder eine Partie Tetris mit mehreren Spieler*innen, die gegeneinander angetreten sind und sich dabei über Chatsysteme auch unterhalten haben, archivieren und über das Archiv auch langfristig ,erleben' und erforschen. Dieses Problem kann hier nicht weiter erläutert werden, allerdings ist es der wichtigste ungeklärte Punkt für langfristige Erforschung und Erhaltung dynamischer digitaler Artefakte, für die bis jetzt noch keine funktionierenden methodischen oder praktischen Konzepte formuliert - und noch viel weniger umgesetzt - werden konnten.

Hier wären die Nationalbibliotheken als jene Institution, die einen nationalen, gesetzlichen Archivierungsauftrag haben, gefordert, die ähnlich wie das privat ins Leben gerufene und von staatlichen Stellen in den USA unterstützte Archive.org diese Aufgabe mit neuer Finanzierung über digitale Medienarchive erfüllen müssten.

Besondere Herausforderungen für sowohl staatliche als auch private digitalen Archivare ergeben sich in den nachfolgenden vier großen Bereichen:

- Legal: Rechtslage teilweise ungeklärt und widersprüchlich, Lizenzen falsch gewählt

- Software: DRM \& Kopierschutz, Abandonware, Betriebssysteme, proprietäre Formate

- Hardware: Peripheriegeräte, Laufwerke, VR-Brillen, diverse Endgeräte, Lagerraum

- Kosten: Initialkosten sowie Kosten zur langfristigen Erhaltung und der Aufrechterhaltung in betriebsfähigen Zustand, Lagerräume

Insbesondere im Rechtsbereich besteht Handlungsbedarf, da dieser den Rahmen für alle Archivierungsbemühungen vorgibt. Denn das klassische Verständnis des Urheberrechts als Ausnahme des Rechts der Allgemeinheit auf Information, Kultur, Bildung und Forschung sowie eines gemeinsamen Bewahrens von Gemeingütern wird zugunsten von Individualrechten und einem absoluten Urheberrechtsansatz zurückgedrängt. Dies bringt Forscher*innen in unhaltbare Rollen.

Diese heute mehr und mehr vorherrschende Sicht gibt Verwerter*innen (Autor*innen bzw. jenen, denen sie die Rechte übertragen haben) eine fast absolute Kontrolle über Inhalte. Dieser Logik folgend hätten wohl Kafkas Werke seinem Wunsch nach verbrannt und nie verlegt werden dürfen - und das ist nur ein Beispiel für den Schaden, den diese von wirtschaftlichen Überlegungen dominierte Herangehensweise ohne ausgewogene Regulierung anrichten kann.

Für die Erforschung ist es unbedingt notwendig, aktiv und zeitnah gerade digitale Artefakte zu archivieren, damit sie überhaupt erforscht werden können. Dies wird durch rechtliche Konstrukte allerdings unmöglich gemacht. Dies wird beispielsweise auch dann deutlich, wenn der deutsche Gesetzgeber explizit ein Recht auf Knacken des Kopierschutzes für die Nationalbibliothek aussprechen muss, um zumindest einige wenige Artefakte rechtmäßig erhalten zu können. Aber selbst dieses Recht umfasst nur die Information, nicht auch die nötige Hardware, um die Information abrufen zu können. 


\section{Retten, was zu retten ist}

Es gäbe in Bezug auf die Erforschung und die damit unmittelbar verbundene Archivierung primär digitaler Quellen noch viel zu sagen, insbesondere auch zum Selbstverständnis und der Praxis von "digital natives“, dem Graubereich der Abandonware, illegalen Archiven, privaten Sammler*innen und der unfreiwilligen Archivfunktion die sogar digitalen Produktpirat*innen zukommen könnte. Dies würde allerdings den Rahmen sprengen und soll deshalb nur als Anregung für weitere Forschungen kurz erwähnt werden.

Trotzdem gibt es abschließend drei einfache Handlungsanweisungen bei digitalen Forschungsprojekten, die Analyse und Archivierung wesentlich erleichtern, wenn sie mitbedacht werden:

- Daten auch in privaten Archiven ablegen: Jedenfalls auch eine private Archivierung bei Projekten einplanen (e.g. archive.org, archiveteam.org)

- Koordination der Archivierung aktiv in Angriff nehmen: Informationen mit Archiven teilen und Archivierung koordinieren, Archivierung mitdenken

- Teilen von Informationen als zusätzliche Kulturtechnik der Langzeiterhaltung: Informationen öffentlich und an mehreren Stellen unter Open Access-Lizenzen online stellen (inkl. Anleitungen, Zusatzdaten etc.)

In diesem Sinne - und auch unter Berücksichtigung der Masse zu archivierender Daten - ist die Lage hoffnungslos, aber nicht ernst.

\section{Literaturverzeichnis}

Cory DoctoRow, Myspace lost all the music its users uploaded between 2003 and 2015, online unter: https://web. archive.org/web/20190409162907/https://boingboing.net/2019/03/17/facebook-is-next.html (09.04.2019).

Domo, Data Never Sleeps 6.0, online unter: https://web.archive.org/web/20190409161923/https://web-assets. domo.com/blog/wp-content/uploads/2018/ (09.04.2019).

Gernot HAUSAR, Weitere Überlegungen zu digitalen Geschichts- und Geisteswissenschaften, in: Julia Menzel, Hg., Digitalität - Theorien und Praktiken des Digitalen in den Geisteswissenschaften 2016, online unter: https://web.archive.org/web/20190409173203/https://digigeist.hypotheses.org/308 (09.04.2019).

Jason HEPPLER, What Is Digital Humanities?, online unter: https://whatisdigitalhumanities.com/ (09.04.2019).

Internet Archive, The Myspace Dragon Hoard (2008-2010), online unter: https://archive.org/details/myspace dragon_hoard_2010 (09.04.2019).

Eric Jou, Two Gamers Are Going To Jail For Stealing $\$ 6,405$ In Virtual Items, online unter: https://web.archive. org/web/20190409160834/ bzw. https://kotaku.com/two-gamers-are-going-to-jail-for-stealing-6-405-invir-1571780985 (09.04.2019).

Henri LefebVRe, Die Produktion des Raums. The Production of Space, Leipzig 2018.

LiNHD, Day of DH, online unter: https://web.archive.org/web/20180828230309/http://dayofdh2016.linhd.es/ (09.04.2019).

Wolfgang SCHMALE, Digitale Geschichtswissenschaft, Köln / Wien 2010. 


\title{
Museumsapps
}

\author{
Individualität versus Plattformstrategie
}

\section{Wolfgang Dorner \& Mariann Juha}

Technische Hochschule Deggendorf

wolfgang.dorner@thd.de / mariann.juha@thd.de

www.thd.de

Keywords: Cultural Tourism, Guide, App, Augmented Reality, Navigation, Platform

\begin{abstract}
This paper aims to analyse the possibilities of information and communication technologies (ICTs) by upgrading the digital experience in cultural tourism and museums through modern and multimedia tools. We challenge the current paradigm of individual apps and compare it to the platform paradigm.
\end{abstract}

In den vergangenen Jahren ist der Anteil besonders an individuellen Museums- und lokalen Kultur-Apps stark gestiegen. Laut Prognosen verbringen die User auch zukünftig immer mehr Zeit mit ihren mobilen Endgeräten. Gleichzeitig zeigen Nutzung bzw. Downloadzahlen sowie Reaktionen von Betreiber*innen ein ernüchterndes Bild: Die User sind immer weniger bereit, weitere Apps herunterzuladen. Wie sieht es im kulturellen Bereich aus? Kann man in dieser Nische methodenkritische Tendenzen erkennen und funktionierende Strategien aufstellen? Benötigen die Kultureinrichtungen individualisierte Apps oder könnten sie in der Zukunft mit Plattformen arbeiten? Nicht zuletzt: Was wünschen sich die Besucher*innen? Wollen sie Apps einzeln herunterladen oder mit der Verwendung von übergeordneten und zusammenfassenden Plattform-Lösungen deren Besuch personalisieren, speichern, über Social Media teilen und die so gespeicherten Informationen wiederverwenden können?

Die Digitalisierung in kulturellen Einrichtungen ist neben Apps auch in Form von Creative Labs, Social Media, Online-Sammlungen und digitalem Besucherbuch immer präsenter. Apps können entweder als reine Media-Guides, als Kreativangebote ${ }^{1}$ (Abb. 1) oder als „all in one“, als eine Art digitales Fenster, interpretiert werden.

1 Wie zum Beispiel die \#ConstructKlee-App (2018), Pinakothek der Moderne, München. Mehr Information zur Ausstellung/ App online unter: https://www.pinakothek.de/klee (22.05.2019). 

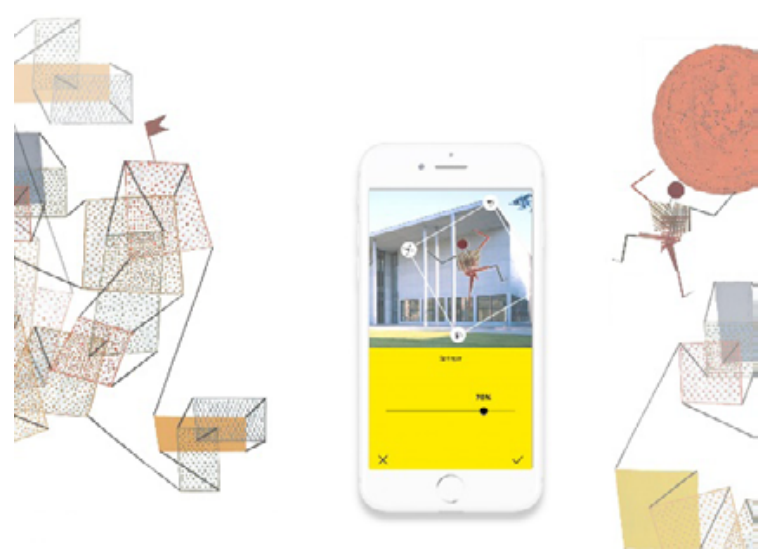

Abb. 1: Beispiel \#ConstructKlee ${ }^{2}$

Durch individuelle multimediale Touren können die Besucher*innen nach ihren persönlichen Interessen und Fähigkeiten die passenden Objekte in der Ausstellung oder, bei lokalen Kultur-Apps, die passenden Orte finden. Wie sollten diese Optionen angeboten werden? Welche Informationen bzw. Anwendungen brauchen die User? Wollen sie überhaupt ihre persönlichen Daten teilen und geführt werden?

Anhand des Beispiels ,Apps als Audio-Guide' soll die Frage gestellt werden, ob museumsindividuelle Lösungen oder Plattformen die geeigneten Strategien sind. Die Technische Hochschule Deggendorf betreut aktuell zwei Kooperationsprojekte, in welchen diese Fragen exemplarisch thematisiert werden. Das erste Projekt ist Peregrinus Silva Bohemica ${ }^{3}$, eine Implementierung eines multimedialen Reiseführers für mobile Geräte, und das zweite ist Museum Uploaded ${ }^{4}$, ein Forschungsprojekt, das die Möglichkeiten der Digitalisierung im musealen Bereich untersucht.

Ziel des Projektes Peregrinus ist es, die historische Kulturlandschaft entlang von grenzüberschreitenden, historischen Wegen im Bayerischen-Böhmischen Wald mit modernen und multimedialen Mitteln für Pilger, Wanderer und geschichtlich Interessierte aufzuwerten. Deshalb steht die Entwicklung eines digitalen Reiseführers als Plattform zur Navigationsunterstützung im Vordergrund (Abb. 2). Historische und kulturelle Themenwege werden über ein Online-Kartenportal verortet und für Web und Smartphone zur Verfügung gestellt. Vorhandene Informationen zu den Denkmälern werden digital neu aufbereitet und interpretiert (z.B. durch 3D Darstellung, AR).

2 DieProduktMacher, Wie man ein Museum schrittweise digitalisiert, online unter: https://www.dieproduktmacher.com/projects/wie-man-ein-museum-schrittweise-digitalisiert (22.05.2019).

3 Das Projekt Peregrinus Silva Bohemica wird durch die Europäische Union Ziel ETZ Freistaat Bayern - Tschechische Republik 2014 - 2020 (Interreg V) durch den Europäischen Fonds für regionale Entwicklung gefördert. Es hat eine Laufzeit von drei Jahren und ist ein gemeinsames Projekt der Westböhmischen Universität Pilsen (Leadpartner), der Beratungsfirma Uhlava und der Technischen Hochschule Deggendorf.

4 Das Projekt Museum Uploaded wird durch die Europäische Union Ziel ETZ Freistaat Bayern - Tschechische Republik 2014-2020 (Interreg V) durch den Europäischen Fonds für regionale Entwicklung gefördert. Es hat eine Laufzeit von drei Jahren und ist ein gemeinsames Projekt der Stadt Deggendorf - Stadtmuseum (Leadpartner), des Prachiner Museum in Pisek, der südböhmischen Universität in České Budějovice und der Technischen Hochschule Deggendorf. 

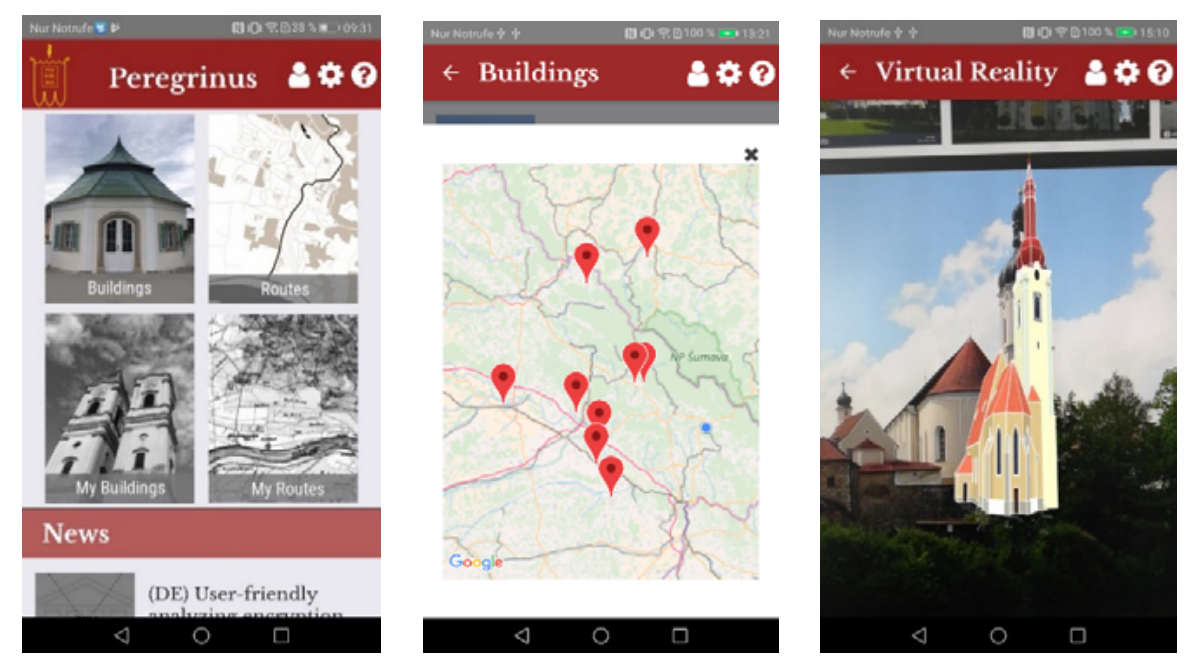

Abb. 2: Mockups zur digitalen Reiseführer-App Peregrinus Silva Bohemica

Unterstützt durch Audio- und Video-Funktionen werden den Interessierten vor Ort anschauliche und detailliertere Informationen bereitgestellt. Auch die Planung und Navigation entlang der historischen Wege wird den Tourist*innen so über den Kartendienst ermöglicht. An einem bestehenden bayerisch-böhmischen Weg aus der Epoche des Barock wird der multimediale Touristenführer exemplarisch aufbereitet und die entwickelten Medien daran getestet. Diese historische Epoche wird damit in der regionalen Tourismuswirtschaft aufgewertet. Die Videoaufnahmen, Audiodaten, Fotografien und Texte werden als Informationsmaterial zu einzelnen Bauwerken und Wegabschnitten angelegt. Mit Usability-Tests wird untersucht, inwieweit die Einbettung der unterschiedlichen Informationen in das Kartenportal für Anwender*innen sinnvoll gestaltet werden kann und einen Mehrwert im Sinne des Nutzer*innen-Erlebnisses bringt. ${ }^{5}$

Die Einbindung von digitalen Medien in der Besucher*innen-Interaktion spielt auch für die moderne Museumsgestaltung eine wichtige Rolle. Die Frage ist, wie die Museen mit den Besucher*innen durch digitale Medien erfolgreich kommunizieren können. Kleinere, lokale Kultureinrichtungen können sich oft keine individualisierten Apps leisten. Wäre eine Plattformlösung die Antwort? Was sollten diese Plattform anbieten? Beim deutsch-tschechischen Kooperationsprojekt Museum Uploaded werden zwei Ansätze verfolgt: Durch Feedbackfunktionen einer geplanten App sollen die Nutzer*innen nicht nur in die Lage versetzt werden, Rückmeldung zu den musealen Inhalten zu geben, sondern auch im Sinne von Zeitzeugenberichten Beiträge zur historischen Forschung liefern können. Kommentarfunktionen, Filter auf diese Kommentare und Social Media-Schnittstellen sollen den Besucher*innen eine breite Möglichkeit bieten, mit dem Museum zu interagieren. So entstehen, neben den von den Museumsmitarbeiter*innen eingegebenen digitalisierten Beständen, Texten, Metadaten und multimedialem Content, neue kollaborative bzw. kuratierte digitale Daten, auf die jeder Zugang hat (siehe Crowdsourcing, Citizen Science, Partizipationsprojekte). Die Museen werden dadurch zu einer ortsunabhängigen 24/7 Datenquelle und können eins gewinnen: Besucher*innen.

$5 \quad$ Zum Beispiel wurde beim Projekt PUP Sight Guide von der Eindhoven University of Technology die zum UNESCO-Weltkulturerbe gehörende Amsterdam als Case Study u.a. für die Untersuchung von 3D-Visualisierungen und AR Implementierungen vorgenommen. Der Fokus lag hier letztendlich nicht auf den visuellen Features, sondern auf dem Vermitteln (mit Hilfe einer personalisierten Führung, Crossovers) und auf der Interaktion mit den Usern (Feedback, GPS-Tracking, Pol); Natalia STASH u.a., The Protected Urban Planet App. „PUP Sight Guide“: Amsterdam as Case Study, in: Regina Franken-Wendelstorf / Elisabeth Lindinger / Jürgen Sieck, Hg., Kultur und Informatik: Visual Worlds \& Interactive Spaces, Glückstadt 2013, 67-80. 
Die Kulturakteure sind sich damit - trotz konservativer Einstellungen ${ }^{6}$ - gemäß einer Kulturtourismus-Studie aus $2018^{7}$ einig, dass ein gemeinsames Profil erarbeitet werden muss. Es wird zwar immer mehr im digitalen Bereich angeboten und die Ausstellungen an die Erwartungen der heutigen Besucher*innen (digital natives kontra digital immigrants) angepasst, auf der anderen Seite fehlt den Museumsmitarbeiter*innen oft das technische Knowhow, damit digitale Angebote bei den Besucher*innen richtig ankommen. Wie die Tabelle 1 zeigt, gibt es noch Möglichkeiten bei der Ausweitung des digitalen Vermittlungsangebotes von Kulturakteuren.

Die einfachste Lösung wäre eine Plattform zur Aufbereitung und Bereitstellung von Inhalten, die leicht bedienbar konzipiert ist und in die gängigen Sammlungsmanagement-Systeme (wie MuseumPlus) integriert werden kann. So würden sich aktuelle Themen aus dem Content Management System (CMS) jederzeit - ohne Zwischenschaltung externer Dienstleister wie Medientechniker - just in time übertragen lassen. Es spricht eigentlich alles für eine Plattform-Lösung, denn individuelle Entwicklungen (wie Museumsapps) sind kostspielige Aktionen - am meisten wegen schlecht einzuschätzender Produktionskosten und Marketingmaßnahmen - mit wenig Download-Zahlen. Sie würde nicht nur die Interaktion zwischen Nutzer*innen und Museen erleichtern.

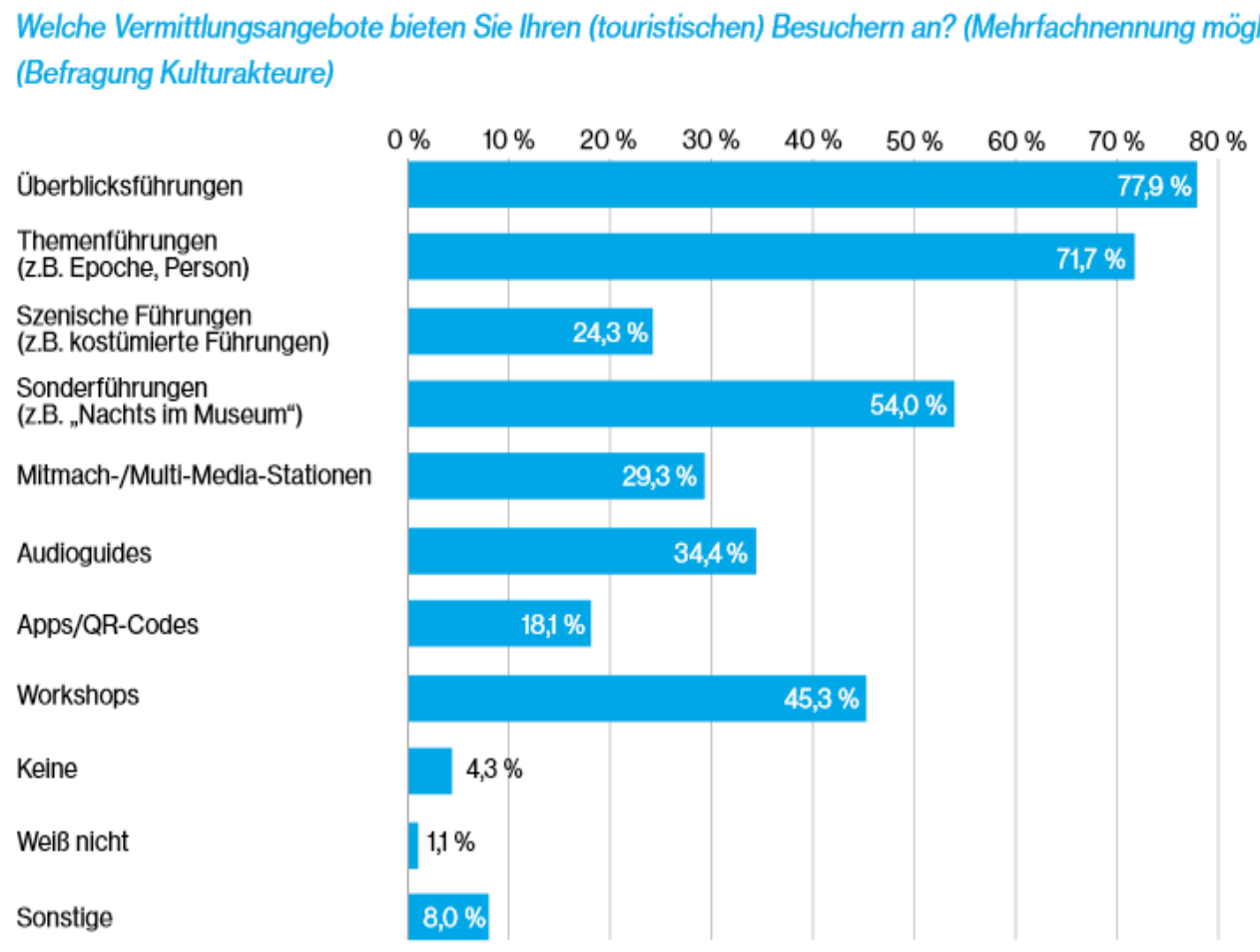

Tabelle 1: Vermittlungsangebote von Kulturakteuren ${ }^{8}$

6 Thomas JERGER, Neues aus den Museen in Oberösterreich, in: Landesstelle für die nichtstaatlichen Museen in Bayern, Hg., 20. Internationale Fachtagung bayerischer, böhmischer, oberösterreichischer und sächsischer Museumsfachleute, Museen bauen und gestalten. 6. bis 8. Oktober 2011, München 2012, 9-25, hier 19.

7 Matthias BurzInSKI / Lara Buschmann / Yvonne PröBstLE, Kulturtourismus Studie 2018. Empirische Einblicke in die Praxis von Kultur- und Tourismusakteuren, online unter: http://projekt2508.de/wp-content/uploads/2018/05/Kulturtourismusstudie-2018-Webversion.pdf (22.05.2019).

8 Ebd., 14 (22.05.2019). 
„45 Prozent der Touristen allgemein werden als aufgeschlossene Entdecker ausgewiesen. Die digitale Vermittlung per Apps oder mobiler Websites sowie Mobile-Marketing sind wichtige Instrumente um diese Zielgruppen zu erreichen. Blogger Relations und WhatsApp-Gruppen müssen genutzt werden. Wichtig ist die Vernetzung der Kultur-Anbieter einer Region zur Verständigung auf ein gemeinsames Ziel oder eine Aussage, um in der Flut der Angebote dem Touristen ein scharfes Bild und Orientierung zu bieten."9

Die User haben wenig Zeit, um die verschiedenen Nutzeroberfläche zu studieren. Die ebenso 2018 im Projekt durchgeführte Befragung zeigt, dass nur wenige der Interviewten die Apps im musealen Bereich kennen, dafür aber erheblich mehr Plattformen im Tourismusbereich (Online-Kartendienste, Hotel-Metasuch-Implementierungen und Touristik-Website). Die überwiegende Mehrheit der Befragten findet, dass eine Plattformlösung wegen Eigenschaften wie Einfachheit, Einheitlichkeit, Nützlichkeit, Übersichtlichkeit, Geschwindigkeit und nicht zuletzt aus zeitlichen Gründen die Lösung sein könnte.

\section{Literaturverzeichnis}

Matthias BURZINSKI / Lara Buschmann / Yvonne PröBstLE, Kulturtourismus Studie 2018. Empirische Einblicke in die Praxis von Kultur- und Tourismusakteuren, online unter: http://projekt2508.de/wp-content/ uploads/2018/05/Kulturtourismusstudie-2018-Webversion.pdf (22.05.2019).

DieProduktMacher, Wie man ein Museum schrittweise digitalisiert, online unter: https://www.dieproduktmacher. com/projects/wie-man-ein-museum-schrittweise-digitalisiert (22.05.2019).

Thomas JeRger, Neues aus den Museen in Oberösterreich, in: Landesstelle für die nichtstaatlichen Museen in Bayern, Hg., 20. Internationale Fachtagung bayerischer, böhmischer, oberösterreichischer und sächsischer Museumsfachleute, Museen bauen und gestalten. 6. bis 8. Oktober 2011, München 2012, 9-25.

N.N., Digitale Trends für Kultur und Tourismus. Stichproben vom Kulturlnvest Kongress Berlin 2016, 08.11.2016, online unter: https://www.icon-design.de/news/digitale-trends-fuer-kultur-und-tourismus (22.05.2019).

Natalia STASH u.a., The Protected Urban Planet App. „PUP Sight Guide“: Amsterdam as Case Study, in: Regina Franken-Wendelstorf / Elisabeth Lindinger / Jürgen Sieck, Hg., Kultur und Informatik: Visual Worlds \& Interactive Spaces, Glückstadt 2013, 67-80.

$9 \quad$ N.N., Digitale Trends für Kultur und Tourismus. Stichproben vom Kulturlnvest Kongress Berlin 2016, 08.11.2016, online unter: https://www.icon-design.de/news/digitale-trends-fuer-kultur-und-tourismus (22.05.2019). 


\title{
Digitising Patterns of Power
}

\author{
An Interactive Map-Based Application to Represent Historical Landscapes
}

\section{Markus Breier [1], Adam Mertel [2], Karel Kriz [1] \& Alexander Pucher [1]}

[1] University of Vienna, Department of Geography and Regional Research markus.breier@univie.ac.at, karel.kriz@univie.ac.at, alexander.pucher@univie.ac.at https://homepage.univie.ac.at/karel.kriz/

[2] Masaryk University, Faculty of Science, Department of Geography mertel.adam@mail.muni.cz

Keywords: Cartographic Communication, Cartographic Visualization, Spatial Uncertainty, HGIS, Historical Landscape

\begin{abstract}
The ÖAW project "Digitising Patterns of Power" (DPP) brings together archaeology, historical research and digital cartography and geocommunication. The communication of space, time and spatial interconnectivity is an essential aspect of the project. An interactive map-based application provides a visual frontend for various aspects of DPP.
\end{abstract}

\section{Introduction}

The perception, depiction and organization of spaces and places in the Middle Ages encompass an interdisciplinary research field which helps to understand historical processes within the medieval period. The representation of space in medieval texts, the appropriation of land and the subsequent installation of new power-structures are central research topics of the project "Digitizing Patterns of Power - Peripherical Mountains in the Medieval World" (DPP). ${ }^{1}$

Patterns of power, established in space and time, are the research focus of DPP. It is a multidisciplinary project, conducted by the Institute for Medieval Research (IMAFO) of the Austrian Academy of Sciences in cooperation with the University of Vienna, Department of Geography and Regional Research (IfGR). The research questions are the domain of historical scholarship, but the phenomena are to a large extent spatial phenomena. The representation and analyses of spatial phenomena are core competences of cartography and geographical information science. The aim of DPP is the development of a generalizable workflow from the digitization of a specific corpus of textual and archaeological evidence to the analysis and visualization of data with the help of digital tools. These requirements led to a map-based online application, which allows the user to visually explore the historical landscape.

Cartography and geocommunication are vital parts in the representation and visualization of the histor-

1 https://dpp.oeaw.ac.at/ (12.06.2019). 
ical landscape and the underlying data. ${ }^{2}$ The creation of project-specific base maps, the visualization of the uncertainty inherent in historical data and the development of methods of interactive geocommunication to create a sustainable online presentation of data and results of the research are central for DPP.

This paper focuses on the geocommunication and visualisation aspects of the project. Other aspects of the project are covered by an edited volume. ${ }^{3}$

\section{Case Studies and historical research questions}

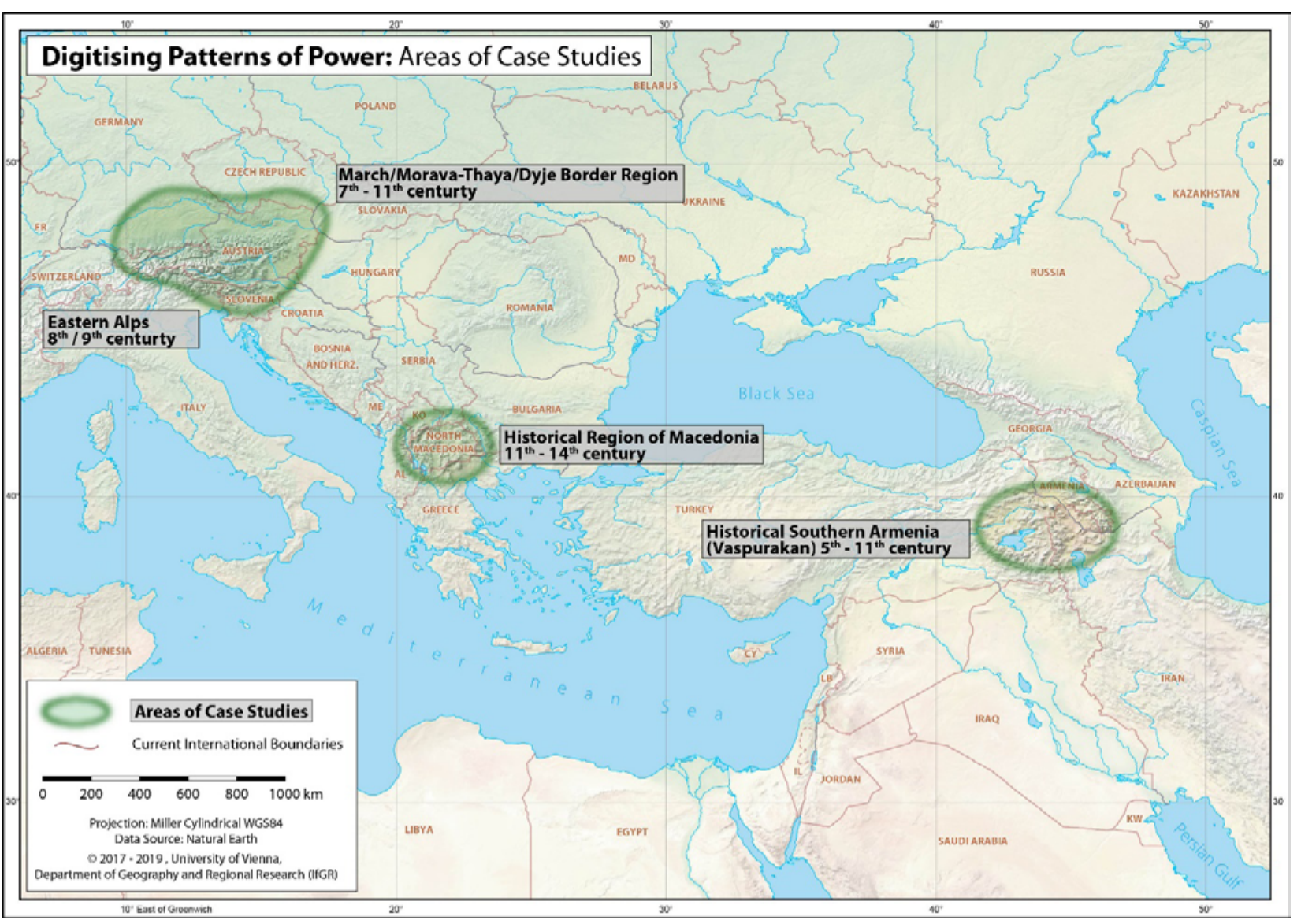

Fig. 1: The case studies of DPP

The project will focus on four case study regions. Historical research questions come from these.

- The Carolingian eastern Alps (8th/9th century)

- The March/Morava - Thaya/Dyje border region (7th-11th century)

- The historical region of Macedonia (12th-14th century)

- Historical southern Armenia: the rise and fall of Vaspurakan (5th-11th century)

2 David J. Bodenhamer / John CORRIGAN / Trevor M. HARRIS, Hg., The spatial humanities. GIS and the future of humanities scholarship, Bloomington 2010; Ian N. GREGORY / Paul S. ELL, Historical GIS. Technologies, methodologies and scholarship, 2. Auflage, Cambridge 2012.

3 Mihailo Popović u. a., Hg., Power in Landscape - Geographic and Digital Approaches in Historical Research, Leipzig 2019. 
The common research topic is the appropriation of space through creating "places of power" and possible underlying strategies.

In the eastern Alps and its surroundings, different structures were established in the late Roman Empire, the Carolingian expansion in the 8th and 9th century and the medieval internal colonization of the Eastern Alps region starting from the 10th century. Ecclesial institutions vied for influence to control the trade and pilgrimage routes to the south. ${ }^{4}$

The Morava/Thaya region is and has always been a border region not only today, but also during the medieval age. The political and social entities on both sides of the border have left certain patterns of power in the landscape. Due to the lack of historical sources in this case study the focus is on archaeological sources. ${ }^{5}$

At the territory of today's North Macedonia, research is conducted on the transformation of the region from a Byzantine province into an area of military and political expansion by the Serbian medieval empire. Impacts on settlement patterns, redistribution of landed property, interplay between resident population and nomads and the establishment of new infrastructure are of interest. ${ }^{6}$

In the historical territory of southern Armenia, research focuses on the region around Lake Van and on the emergence of the principality of the noble house of Arcruni in the period between the end of the ancient Armenian monarchy (428) and the Seljuk conquest of Armenia (1020-1070). A comprehensive analysis of the construction of an early medieval polity at the Van region at the crossroads between Byzantium and the Islamic World is the aim of this case study. ${ }^{7}$

DPP builds on information and data gathered by the project partners from the Academy of Sciences and comprises of archaeological and historical sources. Archaeological entities include artefacts, monuments, settlements, and burial sites; historical information is extracted from written sources like charters, chronicles and travel reports. This information is geotagged and entered in a common database.

\section{Base Maps}

To provide a background for the historical information, a specific base map was created, which suits the needs of the historians and archaeologists. Ideally, the map should represent the landscape at the timeframe appropriate for the research question. However, there were some difficulties to this undertaking. For one, it is very problematic to near impossible to get geodata of the medieval landscape. Not only man-made features like settlements and land use have changed, but also natural features like the course of rivers, coastlines and the extent of lakes have changed during history. Although there has been research on historical courses of some rivers, there is no comprehensive and consistent data

$4 \quad$ Katharina WINCKLER, Die Alpen im Frühmittelalter, Wien 2012.

5 Stefan EICHERT, Frühmittelalterliche Strukturen im Ostalpenraum. Studien zu Geschichte und Archäologie Karantaniens, Klagenfurt am Wörthersee 2012.

6 Mihailo Popović, Vlachen in der historischen Landschaft Mazedonien im Spätmittelalter und in der Frühen Neuzeit, in: Walter Pohl u. a., Hg., Walchen, Romani und Latini: Variationen einer nachrömischen Gruppenbezeichnung zwischen Britannien und dem Balkan, Wien 2017, 183-196.

7 Johannes PREISER-KAPELLER, erdumn, ucht, carayut'iwn. Armenian aristocrats as diplomatic partners of Eastern Roman Emperors, 387-884/885 AD, in: The Armenian review 52 (2010), 139-215; Johannes PREISER-KAPELLER, Networks of border zones: multiplex relations of power, religion and economy in South-eastern Europe, 1250-1453 CE, in: Proceedings of the 39th Annual Conference of Computer Applications and Quantitative Methods in Archaeology, Revive the Past (CAA) in Beijing, China, Amsterdam 2012, 381-393. 
available for all relevant regions. Furthermore, the project spans a timeframe of ten centuries, from the 5th to the 14th century. Overall, it was decided that the base map of DPP is based on current geodata, which will serve as a viable approximation.

To take various prerequisites by different partners and user-groups into consideration, two base maps are available. The default base map is without current borders and cities, focusing on relief, waterbodies and land use. An alternative version is available, which includes the international borders, important cities and the according labels. In higher zoom levels, the current road network is also included. Both base maps are created from free geodata and offer scale-dependent design. Furthermore, the maps of the "Tabula Imperii Byzantini" are integrated as additional map layers.

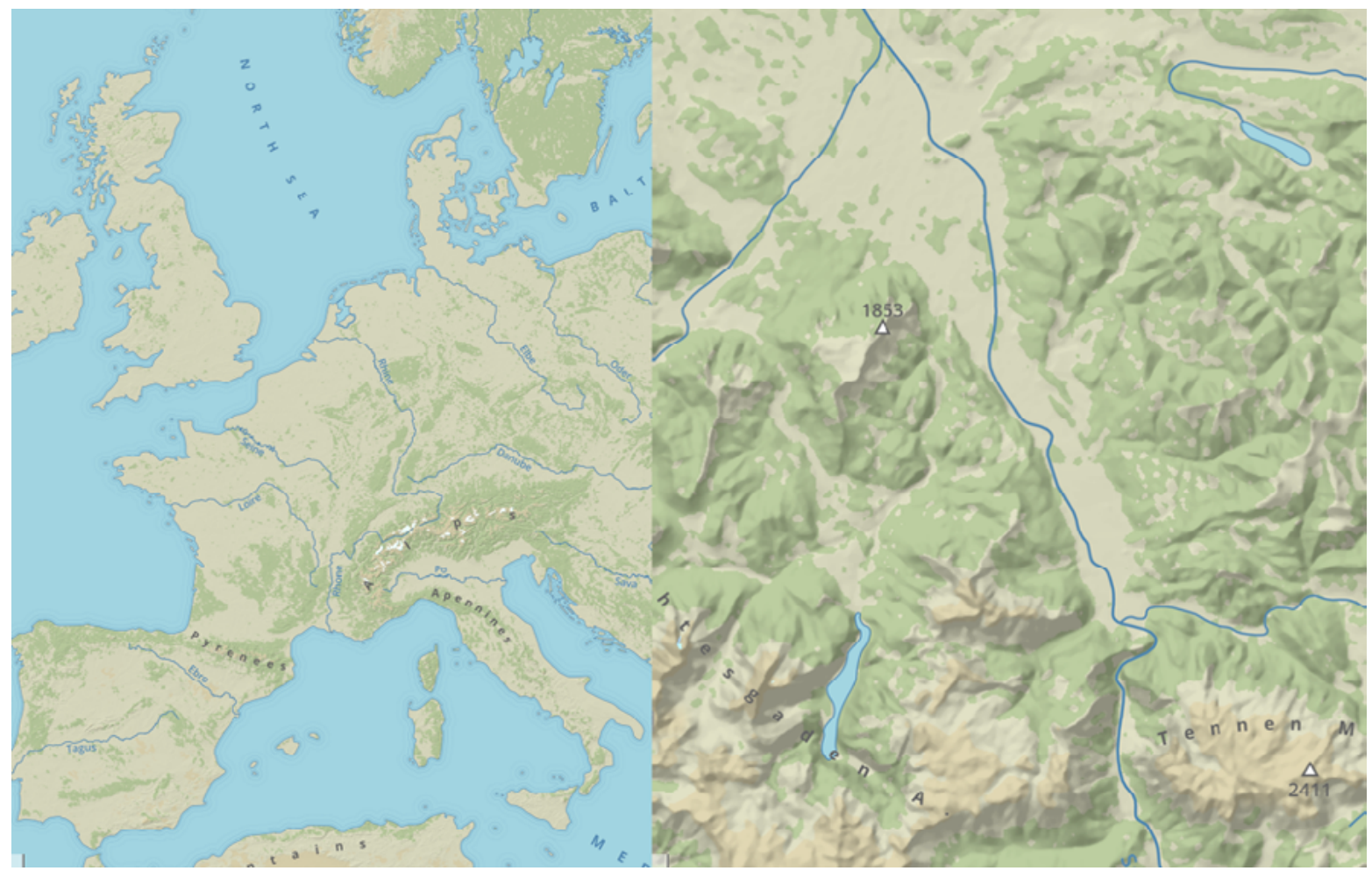

Fig. 2: Base map without modern features. Zoom level 5 (left) and zoom level 11 (right) 


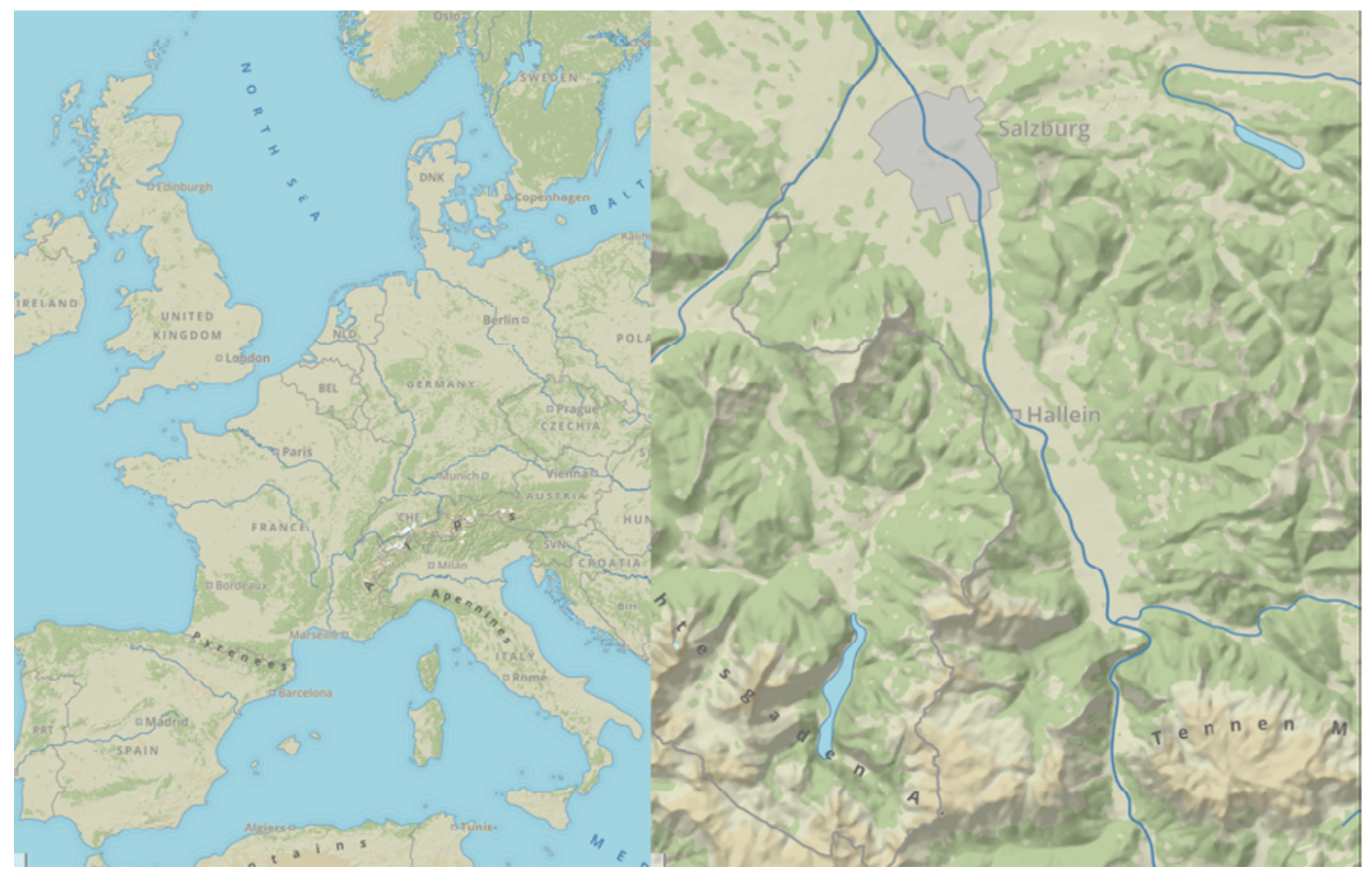

Fig. 3: Base map with modern features. Zoom level 5 (left) and zoom level 11 (right)

\section{Spatial Uncertainty of Historical data}

Due to the nature of the data sources, the spatial data quality varies greatly, especially accuracy and certainty. Data extracted from written sources is most of the times imprecise and uncertain. The references in the written sources are often very vague, e.g. a location was in the vicinity of a town or situated nearby a geographical feature. This is further complicated by the fact that the entity, which the location is referenced to, can be located only vaguely itself. The exact extent of historical administrative units or the area of influence is very hard to determine. Such historical entities were often not clearly defined even during the time they existed. These uncertainties make it difficult to give exact coordinates to the events and locations. Furthermore, the uncertainty has to be represented scale dependent in the map-based application. Approximation methods, like assigning the data to the centre of the current administrative unit or guessing where the location was most likely, are not desirable for this project. Therefore, approaches to make this uncertainty visible were investigated during the project. Although various approaches to uncertainty visualization exist, ${ }^{8}$ these approaches had to be adapted for the use in an interactive application with many different data entities.

In DPP, fuzzy polygons are used to represent uncertain locations. These polygons are an interpretation by the researcher entering the data and are created manually during data input.

8 Alan M. MACEACHREN u. a., Visual Semiotics \& Uncertainty Visualization: An Empirical Study, in: IEEE transactions on visualization and computer graphics 18/12 (2012), 2496-2505. Anne-Kathrin REUSCHEL / Lorenz HURNI, Mapping Literature. Visualisation of Spatial Uncertainty in Fiction, in: The Cartographic Journal 48/4 (2011), 293-308. 


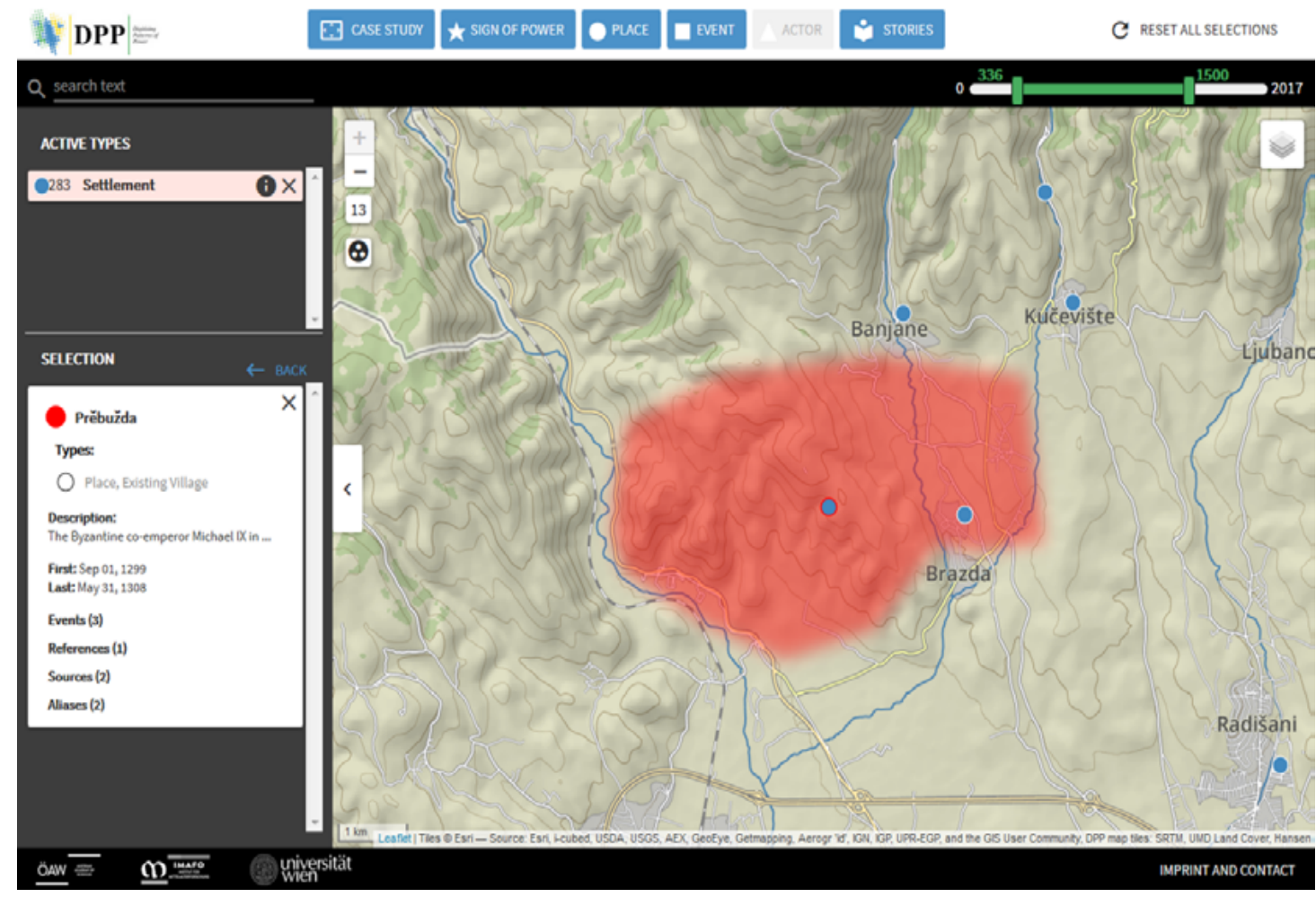

Fig. 5: Visualisation of uncertain locations in DPP

\section{Geocommunication}

The map-based application serves as a tool for research itself. By enabling the user to combine various datasets and results of database queries, spatial relations can be explored.

However, it was not the aim of DPP to create a web-based geographical information system (GIS). DPP focuses on optimal representation of the data and its uncertainty as well as on usability, especially for non-GIS experts and performance. ${ }^{9}$ The application guides the user through the data, allowing to query the database and show various data layers over a purpose made base map.

The application offers a basic query interface for database and navigation to provide a first look at the data available in the database. A time slider allows the user to gain insight to the temporal aspects. For more complex queries, a query builder is available, which allows the user to search and filter for more advanced criteria.

$9 \quad$ Karel KRIZ, Maps and Design-Influence of Depiction, Space and Aesthetics on Geocommunication, in: Karel Kriz u. a., Hg., Understanding Different Geographies, Berlin / Heidelberg 2013, 9-23. 


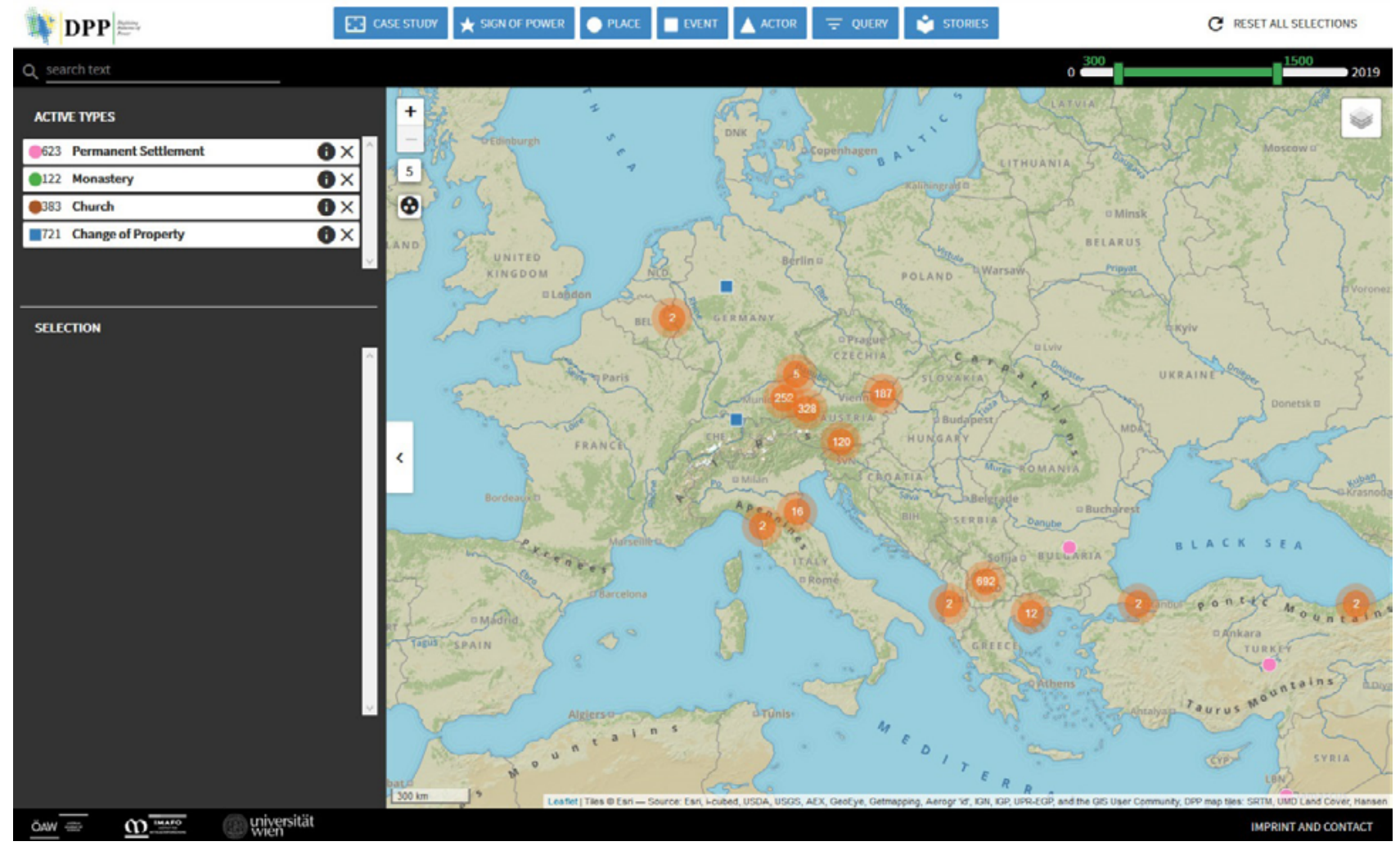

Fig. 6: The map-based application of DPP

Data entities are displayed as dots and are clustered, because there are areas with very high data density. The clustering can be turned off to show the actual distribution of entities. Once an object is selected, the uncertainty information is shown. The links between various data entities like places, events, actors and signs of power can be followed via hyperlinks, which allows to explore relations between entities. With these tools, the data can be explored in its spatial, temporal and thematic aspects to help in answering the historical research question.

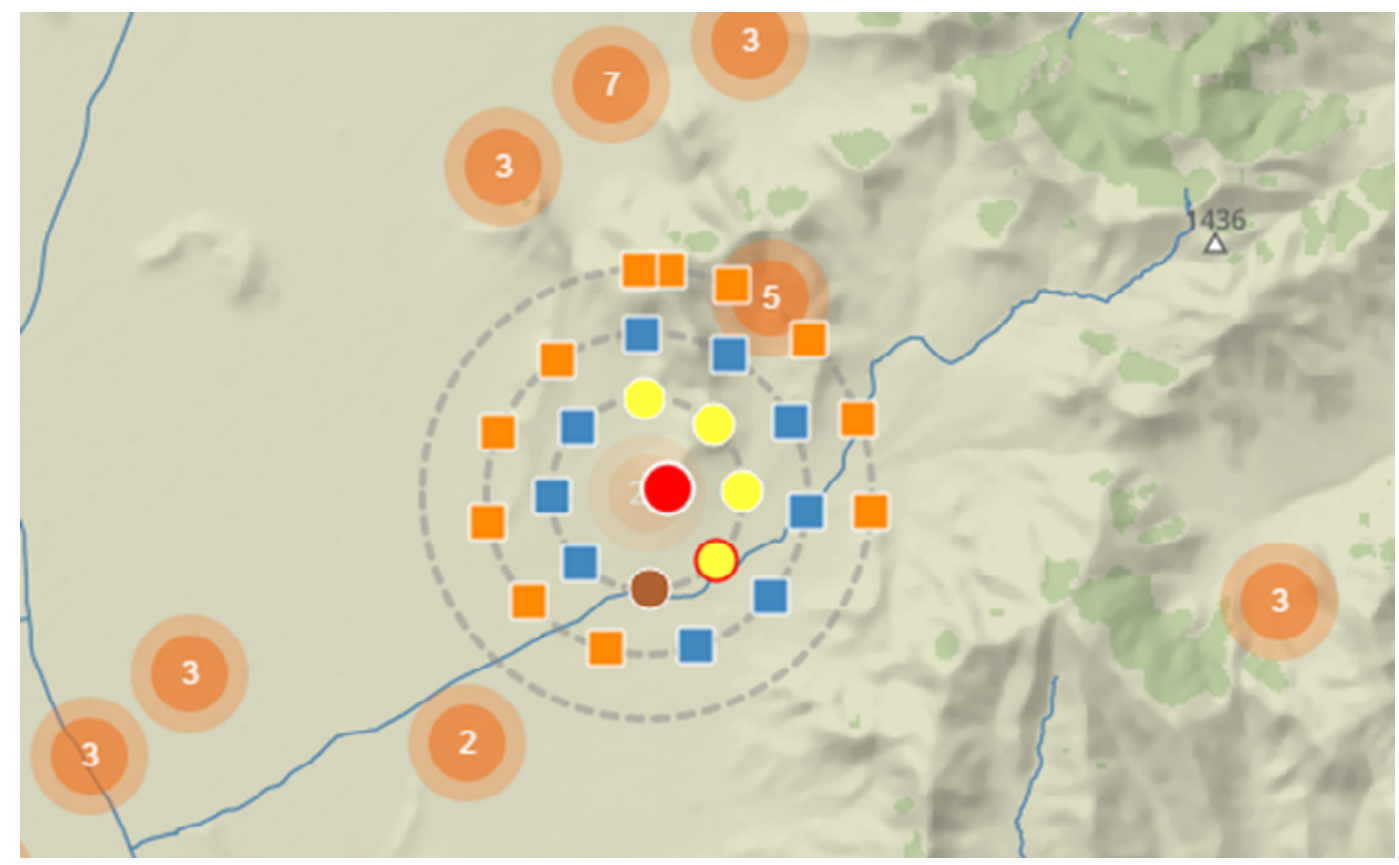

Fig. 7: Clustering in the DPP application 
To communicate key results of the project to the public, so called "Story Maps" are included in the application. These "Story Maps" are predefined views of the data which are complemented with a detailed description of the topic shown and information about its significance for the historiography.

\section{Conclusion}

DPP is a multidisciplinary research project which explores the benefits of state-of-the-art geocommunication technologies to historical research. The focus of the cartographic efforts of the project lies on inherent cartographic issues, which are until now hardly considered in similar projects.

DPP uses high quality base maps, which are created specifically for this project. Story maps and database query functions allow researchers as well as the interested public to browse the data and explore the spatial relations of the entities of the case studies as well as to see the results of the research. The uncertainty of the various entities is also modelled in the database and is represented in the map view.

When designing such a system, usability has to be considered. The aim was to create a flexible system, where the user can conduct her or his own queries and combination of layers. However, it is also easy to use for non-GIS experts. Due to the modular architecture, the framework can be extended with new functionality and used within various similar projects in the future.

\section{Literature}

David J. Bodenhamer / John CoRRIgan / Trevor M. HARRIS, Hg., The spatial humanities. GIS and the future of humanities scholarship, Bloomington 2010.

Stefan EICHERT, Frühmittelalterliche Strukturen im Ostalpenraum. Studien zu Geschichte und Archäologie Karantaniens, Klagenfurt am Wörthersee 2012.

Ian N. GREgory / Paul S. ELL, Historical GIS. Technologies, methodologies and scholarship, 2. Auflage, Cambridge 2012.

Karel KRIZ, Maps and Design-Influence of Depiction, Space and Aesthetics on Geocommunication, in: Karel Kriz u. a., Hg., Understanding Different Geographies, Berlin / Heidelberg 2013, 9-23.

Alan M. MACEACHREN u. a., Visual Semiotics \& Uncertainty Visualization: An Empirical Study, in: IEEE transactions on visualization and computer graphics 18/12 (2012), 2496-2505.

Mihailo Popović, Vlachen in der historischen Landschaft Mazedonien im Spätmittelalter und in der Frühen Neuzeit, in: Walter Pohl u. a., Hg., Walchen, Romani und Latini: Variationen einer nachrömischen Gruppenbezeichnung zwischen Britannien und dem Balkan, Wien 2017, 183-196.

Mihailo Popović u. a., Hg., Power in Landscape - Geographic and Digital Approaches in Historical Research, Leipzig 2019.

Johannes PREISER-KAPELLER, erdumn, ucht, carayut'iwn. Armenian aristocrats as diplomatic partners of Eastern Roman Emperors, 387-884/885 AD, in: The Armenian review 52 (2010), 139-215.

Johannes PREISER-KAPELLER, Networks of border zones: multiplex relations of power, religion and economy in South-eastern Europe, 1250-1453 CE, in: Proceedings of the $39^{\text {th }}$ Annual Conference of Computer Applications and Quantitative Methods in Archaeology, Revive the Past (CAA) in Beijing, China, Amsterdam 2012, 381-393.

Anne-Kathrin REUSCHEL / Lorenz HURNI, Mapping Literature. Visualisation of Spatial Uncertainty in Fiction, in: The Cartographic Journal 48/4 (2011), 293-308.

Katharina WINCKLER, Die Alpen im Frühmittelalter, Wien 2012. 


\title{
The Shape of the Earth
}

\author{
A Round World as Common Worldview within the General Public in the Roman Imperial \\ Period?
}

\section{Sara Wanek}

\author{
Universität Wien, Institut für Klassische Archäologie \\ a00967938@unet.univie.ac.at \\ https://orcid.org/0000-0003-4090-201X
}

Keywords: Round Earth, Astronomical Knowledge, Roman Imperial Period, Worldview

\begin{abstract}
In this paper the material culture and representative archaeological artefacts are used to address the question either if the spherical shape of the Earth was an exclusive knowledge or if it was known by the many in the Roman imperial time.
\end{abstract}

It can be presumed that within the scientific elite a round worldview ${ }^{1}$ was common since the $5^{\text {th }}$ century BCE. ${ }^{2}$ Ancient philosophers and astronomers were aware of this fact, whereat the first written source to mention clear explanations is connected to Aristotle. ${ }^{3}$ Observations of the night sky and of astronomical phenomena indicate that the Earth has to be spherical. ${ }^{4}$ Turning to the time of the Roman Empire, the written records by Claudius Ptolemy dating back in the $2^{\text {nd }}$ century CE provide a profound source for different scientific disciplines including the knowledge of a round shaped Earth. ${ }^{5}$ As usual for classical antiquity written sources mostly reflect the knowledge of a small part of society. ${ }^{6}$ When it comes to the general public in the Roman imperial time the question arises either if the shape of the Earth was an exclusive knowledge, reserved only to a few, or if it was known by the many??

As there are no writings about a general knowledge of the Roman civilization preserved, one has to address this question by consulting the material culture. ${ }^{8}$ Thinking of representative archaeological findings, which are somehow connected to the shape of the world, sundials quickly come to mind. Then again explicit images and representations of globes and stellar spheres have to be taken into consideration.

The term worldview is solely intended to describe the appearance of our planet.

See Olaf BREIDBACH, Geschichte der Naturwissenschaften. Band I: Die Antike, Heidelberg 2015, 270-276.

See Aristoteles, Über den Himmel. Übers. und erl. von Alberto Jori, in: Hellmut Flashar, Hg., Aristoteles Werke in deutscher Übersetzung, Bd. 12, 3, Berlin 2009, 275 f.

4 See BrEIDBACH, Geschichte, 270.

5 See Lennart J. BeRgGRen / Alexander Jones, Ptolemy's Geography. An annotated translation of the theoretical chapters, Princeton 2000, 20-22.

6 See Stephan Busch, Lautes und leises Lesen in der Antike, in: Rheinisches Museum für Philologie 145 (2002), 1-45, here 22-24.

7 The term general public is not used to describe every single person, but it is used to describe the majority of the population within the Roman Empire.

8 See Ernst KüNZL, Himmelsgloben und Sternkarten. Astronomie und Astrologie in Vorzeit und Altertum, Stuttgart $2005,80$. 
Sundials are instruments to record short-term time. Throughout the Roman Empire sundials were a common sight in public and private places, with a vast number appearing in the $1^{\text {st }}$ and $2^{\text {nd }}$ century CE. 9 There has been a widespread distribution of sundials in all regions of the Imperium Romanum, whereby the highest numbers appear in the capital Rome and its surrounding regions (Fig. 1). Time keepers which are based on the daily path of the sun only work correctly on a certain line of latitude. ${ }^{10}$ However, inscribing the latitude on fixed time keepers made of stone was not regular practice. In comparison to that geographical portable sundials show pairings of place names and latitude co-ordinates. Actually, these objects are the only ancient source for the concrete utilization of latitude values besides written sources like Ptolemy's catalogue in the Geographike Hyphegesis. ${ }^{11}$ The knowledge of different lines of latitude is strictly connected not only to a round worldview but also to correct sundials and portable sundials. It is a fact that suggests that the Earth was not thought to be flat but round at least by the producers of sundials and by the owners of the portable ones.

The second group of artefacts which shows a clear connection to the round Earth is made up of specific representations of celestial spheres. When it comes to stellar spheres there are two distinctive examples for the time of the Roman Empire. ${ }^{12} \mathrm{~A}$ detailed marble one is carried by the Atlas Farnese, today on display in the archaeological museum in Naples, Italy. ${ }^{13}$ The other celestial sphere is made of a copper-zinc alloy and it is stored in Mainz, Germany. For both examples the interpretation, dating and assignment of the shown constellations to a certain catalogue are still controversially discussed. ${ }^{14}$ Yet, celestial spheres seem to imply the knowledge of a round shaped Earth. Nevertheless, these two objects have to be related to the upper class and are not representative for the general public.

Furthermore, the globe, which can be depicted as a simple sphere or sphere with crossed lines, was an attribute of the Genius Populi Romani in the period of the Roman Republic (Fig. 2). The attribute also appears in connection with gods, most of all Jupiter. ${ }^{15}$ The first one to use this iconography as personal propaganda was Pompeius. Caesar however was the first one to additionally adopt a godlike position. It is mentioned by Cassius Dio that a bronze statue was placed at the capitol hill in Rome with an inscription naming Caesar as demi-god. This statue is of further interest as Cassius Dio describes moreover that the sphaera underneath his feet was a symbol of the known oikumene, or with other words it was a symbol of the inhabited world ${ }^{16}$. The statue is not preserved but the same iconographical type was also applied by his successor Octavian Augustus. ${ }^{17}$ The image of this statue-type was used as coinage already before 36 BCE. This indicates that images connecting Augustus to autocracy

9 See Eva WINTER, Zeitzeichen. Zur Entwicklung und Verwendung antiker Zeitmesser, Berlin 2013, 14-19; Karlheinz SCHALDACH, Römische Sonnenuhren. Eine Einführung in die antike Gnomonik, Frankfurt am Main 1997, 25-28.

10 See John E. HutH, The Lost Art of Finding Our Way, Harvard 2013, 221-224.

11 See Richard J. A. TALBERT, Roman Portable Sundials. The Empire in Your Hand, Oxford 2017, 111-116.

12 See KüNZL, Himmelsgloben, 6o-65; Elly DEKKER, Illustrating the Phaenomena. Celestial Cartography in Antiquity and the Middle Ages, Oxford 2013, 49.

13 See Javier ARCE / Luis J. BalmasedA, Atlas, in: Jean Ch. Balty u. a., Hg., Lexicon Iconographicum Mythologiae Classicae, Bd.3,1, München 1986, 9-16, here 15-16; Bradley E. ScHAEFER, The Epoch of the Constellations on the Farnese Atlas and Their Origin in Hipparchus Lost Catalogue, in: Journal for the History of Astronomy 36 (2005), 167-196; DEKKER, Phaenomena, 84-102.

14 See KUNZL, Himmelsgloben, 62-63; SCHAEFER, Epoch, 181; Dennis W. DuKE, Analysis of the Farnese Globe, in: Journal for the History of Astronomy 37 (2006), 87-100, here 97; DEKKER, Phaenomena, 69-80.

15 See Tonio HöLSCHER, Victoria Romana. Archäologische Untersuchungen zur Geschichte und Wesensart der römischen Siegesgöttin von den Anfängen bis zum Ende des 3. Jhs. n. Chr., Mainz 1967, 22-24.

16 Cass. Dio $(43,14,6)$ : “(...) And they decreed that a chariot of his (G. J. Caesar) should be placed on the Capitol facing the statue of Jupiter, that his statue in bronze should be mounted upon a likeness of the inhabited world, with an inscription to the effect that he was a demigod (...)".

See Paul ZANKER, Augustus und die Macht der Bilder, München 1990, 49. 
already appeared many years before the Battle of Actium. ${ }^{18}$ Thinking of coins, they can be named as an object class which have been seen and used by the majority of the Roman society. During the Roman Empire coins have to be considered mass media. That is why images on coins were intended for the whole empire and designed for all people. They worked as propaganda for the emperor and for this reason the iconography had to be understood by the vast public. Due to the small size of the images they had to be simple. Only images which could have been easily and universally recognized have been used for coins. ${ }^{19}$ Associated with the victory of Octavian Augustus in the Battle of Actium 31 BCE a new iconographical type using the globe appears on a series of programmatic coins. It is the type of Victoria stepping on a globe. ${ }^{20}$ Initially Victoria occurs mostly in connection with a victory, very often related to a military success. Victoria stepping on a globe is the continuation of this iconography. It was used by Augustus to show his power over the whole empire. ${ }^{21}$ Hölscher describes the usage of Victoria on a globe as follows: "Never before the image of Victoria stepping on a globe would have been more justified than now, as for the first time the finally unified and pacified empire included the whole known world." 22 The globe as attribute of the emperor as well as presentations of the goddess standing on a sphere can be used to demonstrate the large distribution of images of globes throughout the whole Roman Empire. ${ }^{23}$ Soon these new images were adopted by the public. To give an example, this can be noticed by the application of Victoria on a globe on oil lamps. ${ }^{24}$ On coins the iconography is mostly accompanied by the words VICTORIA AVGVSTI or an abbreviation of it. A rather infrequent iconographical type of globes with simplified stars on it starts to occur in the $4^{\text {th }}$ century CE (Fig. 2). ${ }^{25}$

Addressing the controversy either if the spheres are representing stellar spheres or planet Earth it has to be specified that only the globes showing simplified stars have certainly to be interpreted as stellar spheres. The other two types, however, are way more common and sometimes connected to the word ORBIS. Related to imperial representation ORBIS means the inhabitable and dominated world. ${ }^{26}$ Additionally, the passage of Cassius Dio (Cass. Dio 43, 14,6) explicitly names the spheara as oikumene. ${ }^{27}$ Given this, globes on coins are likely to represent first of all the terrestrial sphere. This interpretation seems to apply to Augustus as well as to the subsequent $2^{\text {nd }}$ century CE until probably to the end of the $3^{\text {rd }}$ century. At the beginning of the $4^{\text {th }}$ century there can be noted a shift in the meaning of the spheres towards celestial ones which can be illustrated by the application of simplified stars.

Considering the applied worldview in the Roman imperial period, it can be stated as a fact that the round shape of the Earth was part of the astronomical expertise. As writings mostly reflect the knowledge of a small part of the society it cannot be presumed right away that a round worldview was predominant within the whole Roman civilization. Yet, the round surface of the world causes significant differences on the night sky which can be easily observed by people on the move. Taking in mind the

18 See Zanker, Augustus, 50; M. Bernhart, Die Münzen der römischen Kaiserzeit, München 1944, Taf.1, Abb. 98.

19 See Robert GöBl, Antike Numismatik, Bd.1, München 1978, 25-28; John P. C. Kent / Bernhard OveRBECK / Armin U. StYLOW, Die Römische Münze, München 1973, 7-9.

20 Hereafter I am using Victoria standing and stepping on a globe as synonyms, even though representations of the goddess standing on a sphere with both feet are rather infrequent. See HöLSCHER, Victoria, 17-20; Ursula KampMANN, Die Münzen der römischen Kaiserzeit, Regenstauf 2004, 36-45.

21 See Rainer Vollkommer, Victoria, in: Jean Ch. Balty u. a., Hg., Lexicon Iconographicum Mythologiae Classicae, Bd.8,1, München 1997, 237-269, here 268-269.

22 HÖLSCHER, Victoria, 9.

23 See ibid., 157-172.

24 See ZANKER, Augustus, 265.

25 See HöLSCHER, Victoria, 17-22.

26 See ibid., 43.

27 See ZANKER, Augustus, 49 
expansion of the Roman Empire and the vast number of people travelling, like merchants, soldiers and sailors, it can be assumed that these phenomena have been witnessed by a large number of Roman citizens. Furthermore, it can be stated that in a rural society, as it is valid for the Roman imperial time, astronomical knowledge plays an important role, namely, to help to ensure successful harvest, for orientation on the sea and on the land, and for keeping time. Addressing the material culture there are different groups of artefacts which show a concrete connection to the knowledge of a round world. The vast distribution of sundials seems to imply the transfer of this knowledge. In addition, the appearance of globes, mostly shown as simple spheres or as spheres with crossed lines, in imperial representation and as propaganda for the emperor, significantly support the assumption that a round worldview was applied by a large part of the Roman society in the imperial period. The ongoing study will implement a network analysis using sundials and coins to show the distribution of the knowledge and to address the question of a possible shift of the worldview in the $4^{\text {th }}$ century.

\section{Literature}

Javier ARCE / Luis J. BALMASEDA, Atlas, in: Jean Ch. Balty u. a., Hg., Lexicon Iconographicum Mythologiae Classicae, Bd.3,1, München 1986, 9-16.

Lennart J. BERGgREN / Alexander JONES, Ptolemy's Geography. An annotated translation of the theoretical chapters, Princeton 2000.

M. BERNHART, Die Münzen der römischen Kaiserzeit, München 1944.

Olaf BREIDBACH, Geschichte der Naturwissenschaften. Band I: Die Antike, Heidelberg 2015.

Stephan Busch, Lautes und leises Lesen in der Antike, in: Rheinisches Museum für Philologie 145 (2002), 1-45.

Elly DeKKER, Illustrating the Phaenomena. Celestial Cartography in Antiquity and the Middle Ages, Oxford 2013.

Dennis W. Duke, ANALYSIS OF THE FARNESE GLobe, IN: Journal for the History of Astronomy 37 (2006), 87-100.

Robert GöBL, Antike Numismatik, Bd.1, München 1978.

Tonio HÖLSCHER, Victoria Romana. Archäologische Untersuchungen zur Geschichte und Wesensart der römischen Siegesgöttin von den Anfängen bis zum Ende des 3. Jhs. n. Chr., Mainz 1967.

John E. Huth, The Lost Art of Finding Our Way, Harvard 2013.

Aristoteles, Über den Himmel. Übers. und erl. von Alberto Jori, in: Hellmut Flashar, Hg., Aristoteles Werke in deutscher Übersetzung, Bd. 12, 3, Berlin 2009.

Ursula KampmanN, Die Münzen der römischen Kaiserzeit, Regenstauf 2004.

John P. C. Kent / Bernhard Overbeck / Armin U. StYLow, Die Römische Münze, München 1973.

Ernst KüNZL, Himmelsgloben und Sternkarten. Astronomie und Astrologie in Vorzeit und Altertum, Stuttgart 2005.

Bradley E. ScHAEFER, The Epoch of the Constellations on the Farnese Atlas and Their Origin in Hipparchus Lost Catalogue, in: Journal for the History of Astronomy 36 (2005), 167-196.

Karlheinz SCHALDACH, Römische Sonnenuhren. Eine Einführung in die antike Gnomonik, Frankfurt am Main 1997.

Richard J. A. TALBERT, Roman Portable Sundials. The Empire in Your Hand, Oxford 2017.

Rainer Vollkomer, Victoria, in: Jean Ch. Balty u. a., Hg., Lexicon Iconographicum Mythologiae Classicae, Bd.8,1, München 1997, 237-269.

Eva WINTER, Zeitzeichen. Zur Entwicklung und Verwendung antiker Zeitmesser, Berlin 2013.

Paul ZANKER, Augustus und die Macht der Bilder, München 1990. 


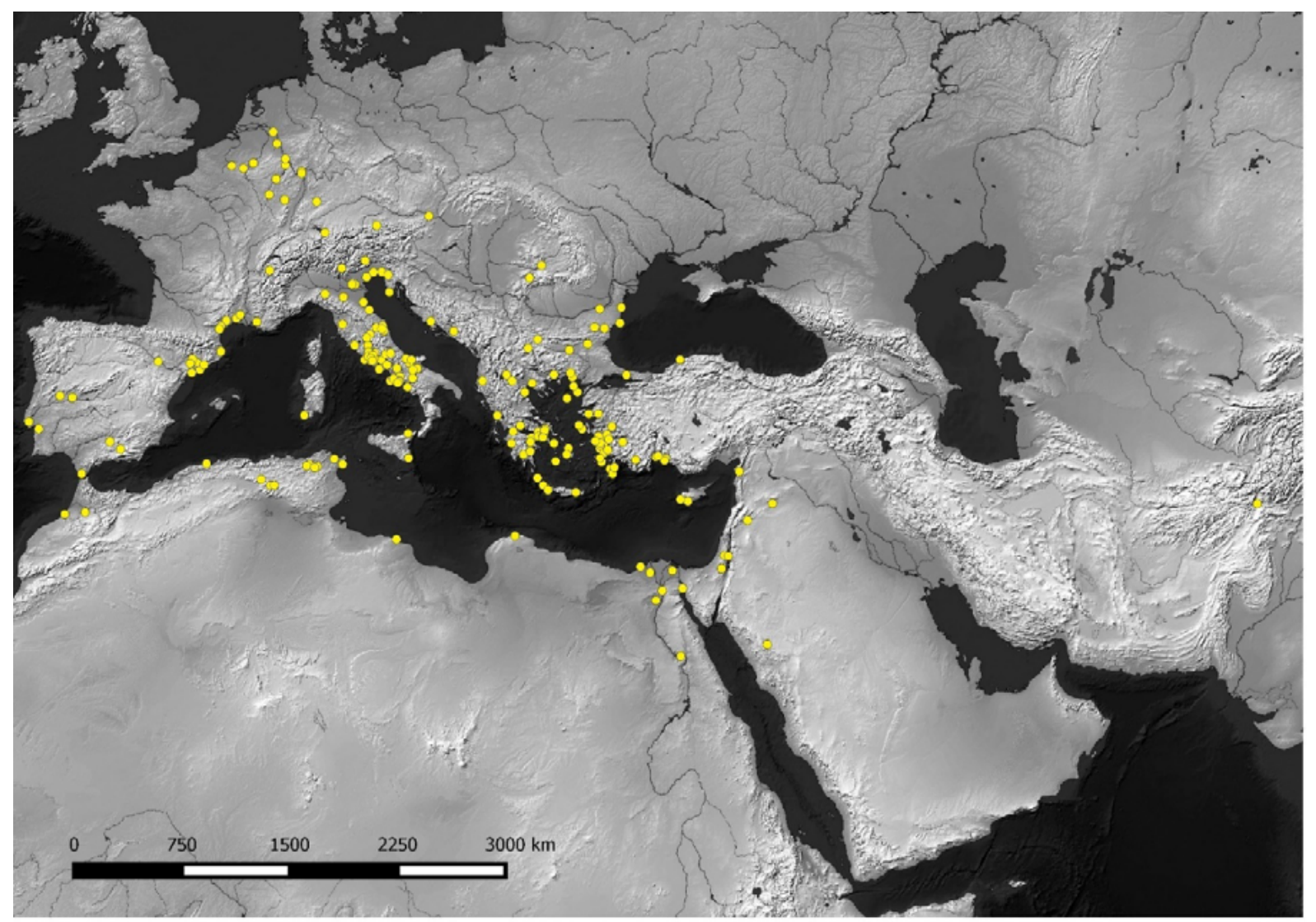

Fig. 1: Distribution of the sundials of E. Winter's catalogue. ( S. Wanek-J. Preiser-Kapeller
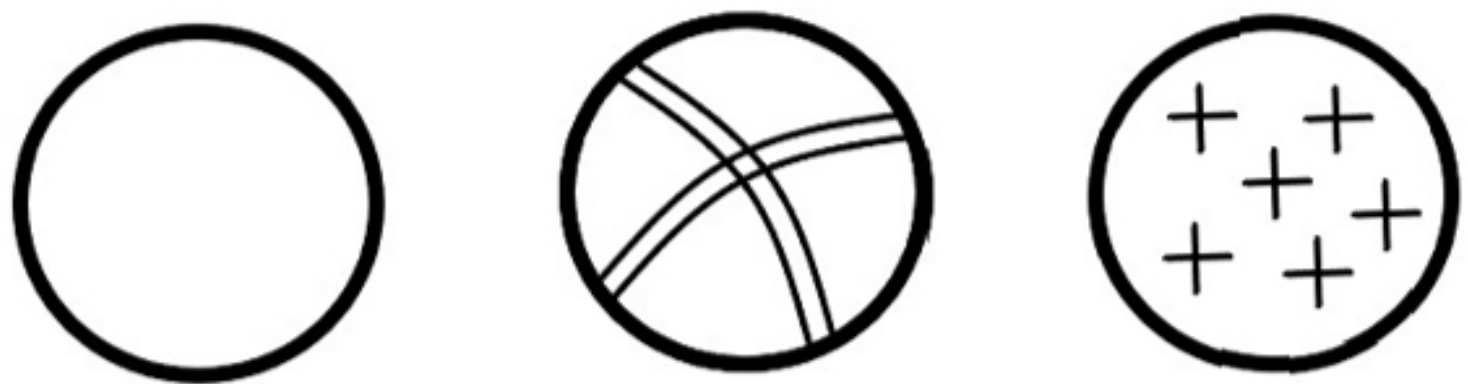

Fig. 2: Division of globes in three types following T. Hölscher. @ S. Wanek 
dial.

\title{
Das Digitale Ilse Aichinger Literaturverzeichnis
}

\author{
Methoden, Quellen und eine erste Auswertung des Literaturverzeichnisses
}

\section{Andreas Dittrich}

Fellow DLA Marbach / Univ. Passau / Bergische Univ. Wuppertal

dittricha@gmail.com

https://orcid.org/0000-0002-1984-7389

Keywords: Ilse Aichinger, Austrian Literature, Bibliography, Wikidata, Bibtex, Biblatex

\begin{abstract}
The Digital Ilse Aichinger List of Literature (dial) lists as many as possible of the published texts of the author Ilse Aichinger (1921-2016) and makes them available via Wikidata.
\end{abstract}

Rosie: Was willst du, Ann?

Ann: Daß wir sie sammeln.

Rosie: Sammeln?

Ann: Und in unseren Händen wärmen, ehe sie verstreut sind.

Rosie: Und wozu?

Ann: Vielleicht, daß dann der Berg hier, der so leuchtet, wie Tomaten nie leuchten würden, verschwindet und Jean wieder am Fenster lehnt. ${ }^{1}$

Das Werk der Schriftstellerin Ilse Aichinger (1921-2016) liegt großteils im S. Fischer Verlag (Frankfurt am Main) publiziert vor. In Wien veröffentlichte vor allem der Verlag Edition Korrespondenzen weitere Texte bzw. versammelte manche der bereits publizierten Texte neu. Damit scheinen die meisten der publizierten Schriften der Autorin leicht zugänglich zu sein. Neben diesen gut erschlossenen Texten gibt es aber noch weitere Texte, die bislang nicht systematisch erfasst wurden. Manche sind der Forschung mehr, andere weniger bekannt, d.h. werden nicht oder nur kaum zitiert. Einige dieser Texte sind mit herkömmlichen Recherchen nur schwer zugänglich, da sie weder katalogisiert, entsprechend beschlagwortet noch auf andere Weise systematisch erfasst worden sind.

Die systematische Erschließung des möglichst gesamten publizierten Text-Korpus ist eine wichtige Grundlage für die Auseinandersetzung mit dem literarischen Gesamtwerk einer Autorin bzw. eines Autors. Diese manifestiert sich herkömmlicherweise in Form der subjektiven Personal-

1 Ilse AichingeR, Knöpfe, in: Dies., Auckland. Hörspiele, Frankfurt am Main 1991, 11-73, hier 52. 
bibliografie ${ }^{2}$ und ist für das Werk Ilse Aichingers bislang nicht erschöpfend geleistet worden: Die bislang vorgelegten Bibliografien von Julia Karnahl mit $32,{ }^{3}$ von Ingrid Gomboz mit $85^{4}$ und von Richard Reichensperger mit 111 Einträgen ${ }^{5}$ sind unvollständig. Das Digitale Ilse Aichinger Literaturverzeichnis (dial) verzeichnet (von derzeit insgesamt 1.865 bibliografischen Einheiten) rund 662 Erstpublikationen (Stand Juli 2019). Die große quantitative Diskrepanz zwischen den bisherigen Bibliografien und dem dial entsteht zunächst durch den Umstand, dass die Bibliografien nicht Texte, sondern Bücher verzeichnen. Das dial hingegen erfasst einzelne Texte, ${ }^{6}$ löst sich in gewisser Weise von der Einheit des Buches und reflektiert dieses durch das abwesende biblos im Namen. Davon abgesehen befinden sich im dial derzeit aber auch 66 bislang nicht in den bisherigen Bibliografien verzeichnete Texte.

Der primäre Zweck des Projekts dial war es zunächst, ein möglichst umfangreiches Verzeichnis aller publizierten Texte der Autorin zu erstellen um damit auch jenen Texten eine Sichtbarkeit zu geben, die sich bislang nicht am Horizont der Forschung befanden. Das Prinzip der Vollständigkeit ist dabei aber nur tentativ zu verstehen, da stets neue Auflagen oder gar ,neue‘ Publikationen (beispielsweise aus dem Nachlass) erscheinen können. ${ }^{7}$ Dieser Umstand war mit ein Grund dafür, das Verzeichnis mit digitalen Hilfsmitteln zu erstellen und über das World Wide Web frei und offen zur Verfügung zu stellen, so dass es erstens jederzeit aktualisiert bzw. korrigiert werden und zweitens leicht und flexibel (,multidimensional“8) durchsucht werden kann. ${ }^{9}$

\section{Quellen}

In das dial wurden ausschließlich gedruckt publizierte Texte aufgenommen, die unter dem Namen von Ilse Aichinger publiziert wurden bzw. auf sie rückführbar sind und keine Übersetzungen darstellen. Das impliziert - neben den herkömmlich publizierten Texten in Büchern, Sammelbänden, Zeitschriften und Zeitungen - auch (nicht zur Publikation intendierte, aber dennoch publizierte) Briefe ${ }^{10}$, später aus dem Nachlass publizierte Texte oder auch Interviews, die mit ihr geführt und abgedruckt wurden. Nicht aufgenommen wurden Inszenierungen ihrer Texte (als Hörspiel oder Film), Radio- oder Fernseh-Interviews oder Filme mit ihr. Die Kataloge und Bestände folgender Bibliotheken und Archive wurden konsultiert:

- der Österreichische Verbundkatalog,

- der Katalog der Deutschen Nationalbibliothek,

2 Vgl. Dirk WISSEN / Konrad UMLAUF, Subjektive Personalbibliografie, in: Konrad Umlauf / Stefan Gradmann, Hg., Lexikon der Bibliotheks- und Informationswissenschaft, Stuttgart 2014, 863.

3 Julia KARNAHL, Auswahlbibliographie, in: Text + Kritik: Zeitschrift für Literatur 175 (2007), 112-115.

4 Ingrid Gomвоz, Bibliographie Ilse Aichinger, in: Kurt Bartsch / Gerhard Melzer, Hg., Ilse Aichinger, Graz 1993, $249-293$.

5 Richard Reichensperger, Bibliographie der Werke Ilse Aichingers, in: Samuel Moser, Hg., Ilse Aichinger. Leben und Werk: Informationen und Materialien zur Literatur. Aktualisierte und erweiterte Neuausgabe, Frankfurt am Main 1995, 345355 .

6 Die Komposition der von Aichinger publizierten Bücher wird im dial auch als Text und damit als eigenes Werk verstanden.

7 Vgl. Friedrich NestLeR / Georg SchneIDER, Einführung in die Bibliographie, Stuttgart 2005, 67: „Vollständigkeit ist stets ein angestrebtes Ziel innerhalb konzeptionell festgelegter Grenzen.“ Vgl. auch Friedrich DomAY, Formenlehre der bibliographischen Ermittlung. Eine Einführung in die Praxis der Literaturerschließung, Stuttgart 1968, 109.

8 NESTLER / SCHNEIDER, Einführung, 66.

9 Vgl. ebd., 27; Vgl. auch Laura Marie PohlmanN, Von Quellen und Gefäßen. Die neue Alfred Döblin-Bibliographie, in: Sabina Becker / Sabine Schneider, Hg., Internationales Alfred-Döblin-Kolloquium Zürich 2015, Frankfurt am Main 2017, 333-343.

10 So zum Beispiel die Briefe an Gottfried und Brigitte Bermann-Fischer in Reiner STACH / Karin ScHLAPP, Hg., Gottfried Bermann-Fischer / Brigitte Bermann-Fischer: Briefwechsel mit Autoren, Frankfurt am Main 1990, 531-544. 
- das kommentierte Verzeichnis der Literaturzeitschriften in Österreich 1945-1990 der ÖNB, ${ }^{11}$

- die Bibliothek der Arbeiterkammer in Wien,

- das Innsbrucker Zeitungsarchiv (sowohl online als auch vor Ort),

- das Otl Aicher Archiv der Hochschule für Gestaltung in UIm,

- die Zeitschriftendatenbank ZDB,

- der KVK - Karlsruher Virtueller Katalog,

- verschiedene Bibliografien und Bibliografien von Bibliografien, ${ }^{12}$

- vor allem das Deutsche Literaturarchiv Marbach (DLA Marbach), wo, neben dem Nachlass von Ilse Aichinger und dem ihres Mannes Günter Eich, ${ }^{13}$

- das S. Fischer Verlagsarchiv,

- eine reichhaltige Mediendokumentation,

- die Kontobücher der (einige Texte von Aichinger vermittelnden) Literaturagentur Ruhr-Story ${ }^{14}$ und

- der fein differenzierte und sehr hilfreiche Katalog Kallías zur Verfügung stehen.

Zusätzlich waren die durchsuchbaren digitalen Korpora und Datenbanken folgender Institutionen hilfreich:

- das AAC-Austrian Academy Corpus der Österreichischen Akademie der Wissenschaften, ${ }^{15}$

- ANNO - AustriaN Newspapers Online der Österreichischen Nationalbibliothek,

- die WISO Datenbank,

- die Webpräsenz der Tageszeitung Der Standard,

- das digitale Neue Rundschau Archiv und

- das digitalisierte Volkshochschul-Programmarchiv des Deutschen Instituts für Erwachsenenbildung / Leibniz-Zentrum für Lebenslanges Lernen e.V. ${ }^{16}$

11 https://www.onb.ac.at/oe-literaturzeitschriften/ (22.05.2019).

12 Unter den Bibliografien sollen hier folgende Publikationen hervorgehoben werden: Hörspielsendungen verzeichnet Gisela LINDEMANN, Ilse Aichinger, München 1988. Übersetzungen werden, neben der in der erwähnten GOMBOZ-Bibliografie, auch bei Irmela HoltMEIER, in: Ansprachen und Dokumente zur Verleihung des Kulturpreises der Stadt Dortmund, Nelly-Sachs-Preis, Dortmund 1971, 49-55 verzeichnet.

13 Aichingers und Eichs gemeinsame (und vermutlich nicht ganz vollständige) Autorenbibliothek befindet sich im DLA Marbach und besteht aus 274 Bänden (3 Achsen), die großteils Belegexemplare von Übersetzungen (mehrheitlich von Eichs Büchern) sind.

14 Die Kontobücher sind am DLA Marbach im Bestand A:Ruhr-Story/Kontobücher zu finden. Vgl. dazu den Beitrag von Ulrich von BüLow, Im Netzwerk. Das Deutsche Literaturarchiv in Marbach hütet von nun an den Nachlass von Siegfried Lenz, https://web.archive.org/web/20180829170822/https://www.kulturstiftung.de/im-netzwerk/ (29.08.2018).

15 http://www.aac.ac.at/ (22.05.2019).

16 https://www.die-bonn.de/ (22.05.2019). 


\section{Aufbau}

Die bibliografischen Angaben wurden zunächst im Format Biblatex erfasst, welches ein fein differenziertes Vokabular zur Beschreibung der bibliografischen Elemente bietet, ${ }^{17}$ und als menschen- sowie maschinenlesbare Textdatei gespeichert. Die Datei wird im Projekt ARCHE der ÖAW archiviert ${ }^{18}$ und im Internet zugänglich gemacht. ${ }^{19} \mathrm{Um}$ die jeweils als gleich angesprochenen Texte miteinander in Beziehung zu setzen - hier folgt und umgeht das dial in gewisser Weise die FRBR-Idee ${ }^{20}$-, wurden diese mit einer identischen Identifikationsnummer, der entsprechenden Wikidata-ID, versehen. ${ }^{21}$ Diese sind nun per SPARQL-Abfrage bzw. über die semi-visuelle Oberfläche des Wikidata Query Service abfrag- und visualisierbar. ${ }^{22}$

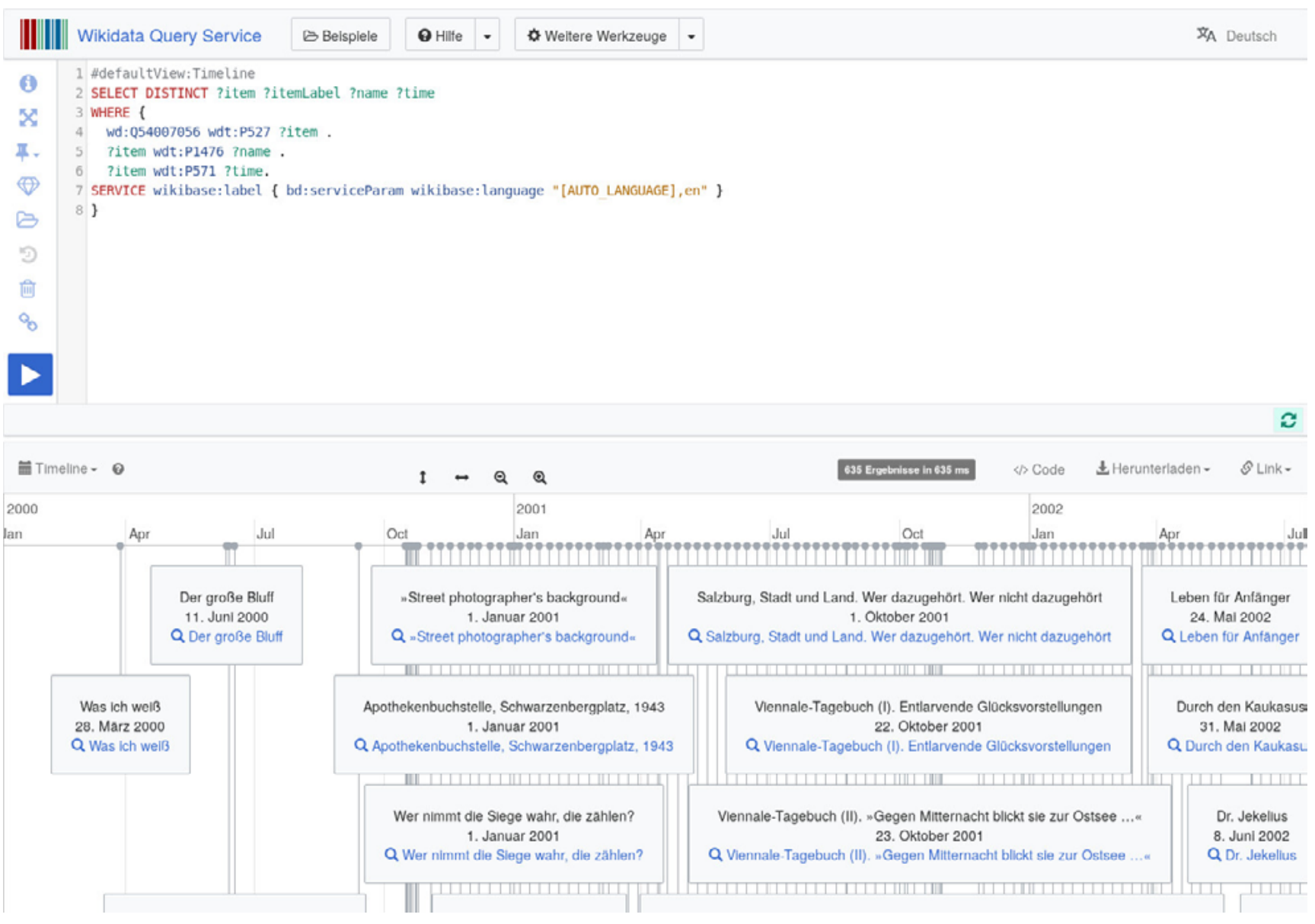

Abb. 1: Über die Seite query.wikidata.org kann das dial beispielsweise auch auf einer Timeline dargestellt werden, siehe: https://w.wiki/624.

17 https://github.com/plk/biblatex (22.05.2019).

$18 \mathrm{https} / / /$ id.acdh.oeaw.ac.at/dial (01.09.2019).

$19 \mathrm{http}: / /$ dial.aichingerhaus.at/ (22.05.2019).

20 Die Functional Requirements for Bibliographic Records (FRBR) unterscheiden zwischen work, expression, manifestation und item. Die Werk-Einheiten werden dabei (in einer metaphysischen Form) als abstrakt-immaterielle Objekte angesetzt. Das dial verbindet bewusst die jeweiligen, miteinander in Beziehung stehenden Texte, ohne ein solches abstrakt-immaterielles Objekt zu konstruieren: es bleibt bei den jeweiligen konkreten bibliografischen Einheiten.

21 Das ist in Wikidata unter https://www.wikidata.org/wiki/Q54007056 (22.05.2019) versammelt.

22 https://query.wikidata.org/ (22.05.2019). 


\section{Analysen}

Ein erster Gewinn der Arbeit am dial besteht darin, bislang kaum bekannte Texte entdeckt zu haben. So verwendet Aichinger in einer frühen Theater-Rezension bereits das Motiv des Vergessens ${ }^{23}$ oder reflektiert Zeitlichkeit in einem bislang nicht wieder aufgelegten kurzen Prosagedicht. ${ }^{24}$

Davon abgesehen, lässt sich auf Grundlage des dial aber auch feststellen, welche Texte Aichingers am häufigsten wieder abgedruckt wurden („Die größere Hoffnung“, 28 mal). Der Text „Vor der langen Zeit" wurde oft in Tageszeitungen und zu Weihnachten wieder abgedruckt (21 mal); „Die Silbermünze“ ist zwar relativ häufig publiziert worden (16 mal), wurde aber nicht in die Ausgabe „Werke in acht Bänden“ (1991) aufgenommen. Auffallend ist, dass sich mit "Gebirgsrand“ (14 mal), welches das Buch „verschenkter Rat“ eröffnet, auch ein Gedicht unter den zehn am häufigsten wieder abgedruckten Texten befindet. Jedes Feld des strukturierten Datensatzes lässt sich quantitativ auswerten. Nunmehr lässt sich empirisch fundiert sagen, dass der Großteil der Texte Aichingers in Deutschland (hier vor allem bei S.Fischer) verlegt wurde. Und das ist nur ein Beispiel.

Das strukturierte Format eines Literaturverzeichnisses bietet sich besonders für eine digitale Repräsentation an. Dennoch muss auch bedacht werden, dass, in Anbetracht der häufigen Ausrufung neuer Standards, für die Persistenz der Daten auch eine Drucklegung in Betracht gezogen werden sollte.

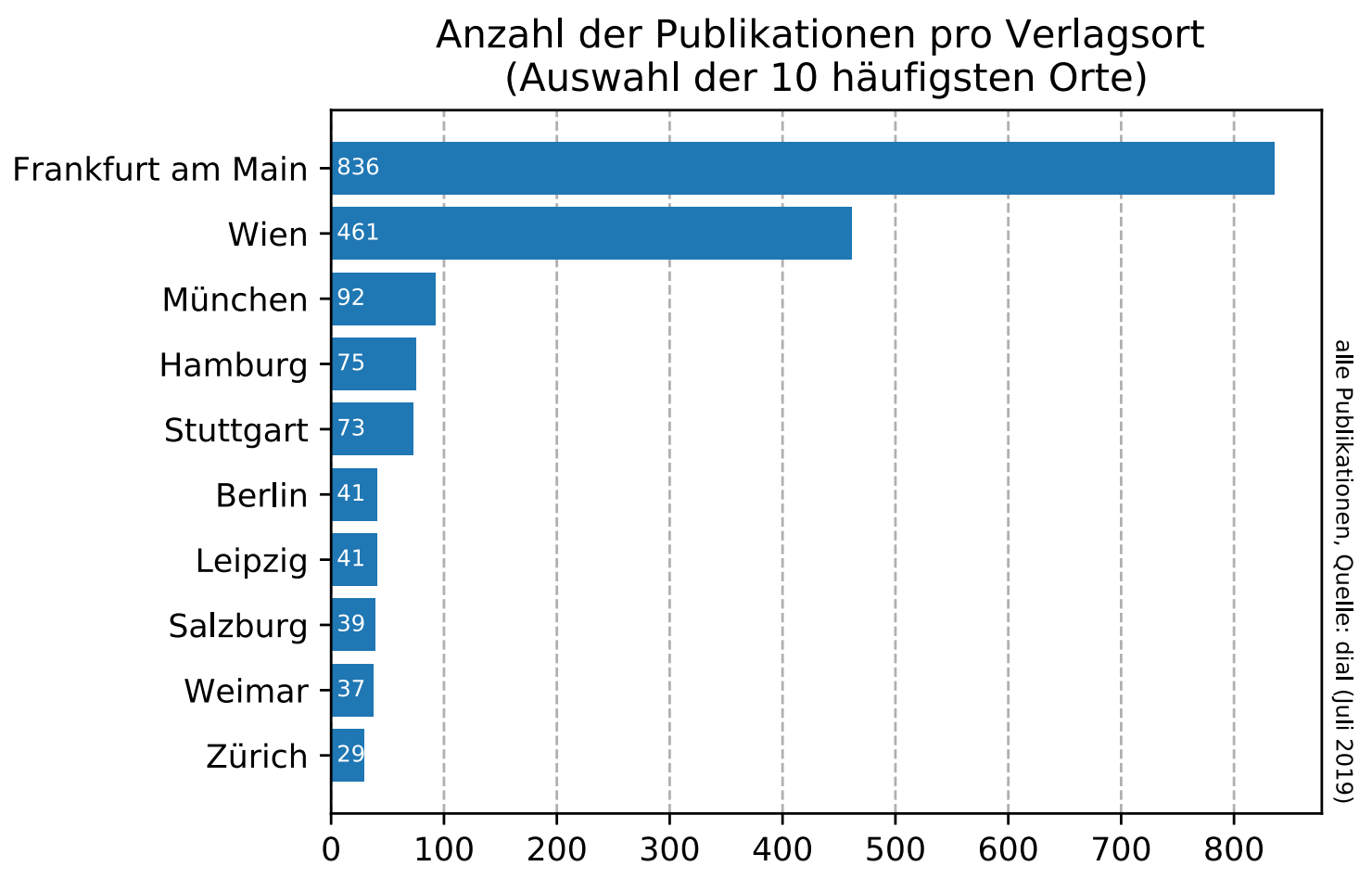

Abb. 2: Die zehn häufigsten Verlagsorte im dial (Stand Juli 2019).

23 Ilse AICHINGER, Der Londoner ,Verlorene Sohn'. Theater der Stephansspieler, in: Der Turm 2/3-4, Wien 01.03.1946, $161 f$.

24 Ilse AICHINGER, Der Schreibtisch des kleinen Michele B., in: Deutsche Zeitung, Köln 20./21.08.1960, 19. 


\section{Danksagung}

Das Projekt wurde im Rahmen eines Stipendiums vom Forschungsverbund Marbach Weimar Wolfenbüttel (MWW) finanziell ermöglicht. Am DLA Marbach bin ich für die Anregungen, die ich bei der Präsentation im Rahmen des Mittwochsseminars bekommen habe, und die Unterstützung, die ich von Janet Dilger, die die Daten derzeit peu à peu in die GND einarbeitet, dankbar. Martina Trognitz (ÖAW) möchte ich für die tatkräftige technische Unterstützung danken. Hanno Biber (ÖAW), der das Projekt nicht nur kreativ gefördert hat, und Christine Ivanovic (Univ. Wien), ohne deren Unterstützung es erst gar nicht zustande kommen hätte können, möchte ich meinen herzlichen Dank aussprechen.

\section{Literaturverzeichnis}

IIse AICHINGER, Der Londoner, Verlorene Sohn'. Theater der Stephansspieler, in: Der Turm 2/3-4, Wien 01.03.1946, $161 f$.

Ilse AICHINGER, Der Schreibtisch des kleinen Michele B., in: Deutsche Zeitung, Köln 20./21.08.1960, 19.

Ilse AICHINGER, Knöpfe, in: Dies., Auckland. Hörspiele, Frankfurt am Main 1991, 11-73.

Friedrich DomaY, Formenlehre der bibliographischen Ermittlung. Eine Einführung in die Praxis der Literaturerschließung, Stuttgart 1968.

Ingrid Goмвоz, Bibliographie Ilse Aichinger, in: Kurt Bartsch / Gerhard Melzer, Hg., Ilse Aichinger, Graz 1993, 249-293.

Julia KARNAHL, Auswahlbibliographie, in: Text + Kritik: Zeitschrift für Literatur 175 (2007), 112-115.

Friedrich NESTLER / Georg SCHNEIDER, Einführung in die Bibliographie, Stuttgart 2005.

Laura Marie PohlmanN, Von Quellen und Gefäßen. Die neue Alfred Döblin-Bibliographie, in: Sabina Becker / Sabine Schneider, Hg., Internationales Alfred-Döblin-Kolloquium Zürich 2015, Frankfurt am Main 2017, 333-343.

Richard ReICHENSPERgER, Bibliographie der Werke Ilse Aichingers, in: Samuel Moser, Hg., Ilse Aichinger. Leben und Werk: Informationen und Materialien zur Literatur. Aktualisierte und erweiterte Neuausgabe, Frankfurt am Main 1995, 345-355.

Reiner STACH / Karin SCHLAPP, Hg., Gottfried Bermann-Fischer / Brigitte Bermann-Fischer: Briefwechsel mit Autoren, Frankfurt am Main 1990.

Dirk WISSEN / Konrad UMLAUF, Subjektive Personalbibliografie, in: Konrad Umlauf / Stefan Gradmann, Hg., Lexikon der Bibliotheks- und Informationswissenschaft, Stuttgart 2014.

https://www.onb.ac.at/oe-literaturzeitschriften/ (22.05.2019).

https://www.die-bonn.de/ (22.05.2019).

https://github.com/plk/biblatex (22.05.2019).

http://dial.aichingerhaus.at/ (22.05.2019).

https://id.acdh.oeaw.ac.at/dial (01.09.2019). 


\title{
Die Digitale Mozart-Edition (DME)
}

\author{
Interdisziplinäre Projekte unter einem Dach
}

\author{
Mirijam Beier \& Till Reininghaus \\ Internationale Stiftung Mozarteum, Salzburg \\ beier@mozarteum.at, reininghaus@mozarteum.at \\ https://mozarteum.at/
}
Keywords: Music Encoding Initiative (MEI), Text Encoding Initiative (TEI), Digital Edition, Interdisciplinarity, Wolfgang Amadé Mozart

\begin{abstract}
The Digital Mozart Edition unites various, interdisciplinary and freely accessible projects under one roof. All subprojects, like DME::Letters \& Documents or DME::Music, aim at ensuring a versatile and long-term reuse and therefore are based on XML formats like TEI and MEI.
\end{abstract}

Die Digitale Mozart-Edition (DME) ist ein an der Internationalen Stiftung Mozarteum (ISM) in Salzburg angesiedeltes Langzeitprojekt, das dort gemeinsam mit dem in Los Altos, Kalifornien/USA, beheimateten Packard Humanities Institute $(\mathrm{PHI})$ durchgeführt wird. Unter dem Dach der DME sind verschiedene Teilprojekte versammelt, deren gemeinsames Ziel es ist, das gesamte Schaffen des Komponisten Wolfgang Amadé Mozart (1756-1791) in seinen vielfältigen Facetten digital aufzubereiten und im Internet für nicht-kommerzielle Zwecke unentgeltlich zur Verfügung zu stellen. ${ }^{1}$ Hierdurch sollen Ergebnisse der Forschung einem möglichst großen Kreis von Interessierten, z.B. Wissenschaftler*innen, Musiker*innen oder Lehrer*innen, aber auch Mozart-Liebhaber*innen, zugänglich gemacht werden. Um eine vielfältige und langfristige Nachnutzung zu ermöglichen, verwendet die DME XMLbasierte Formate wie TEI und MEI.

Im Projekt DME::Librettos \& Texts bietet die DME diverse Editionen von historischen Publikationen, die mit Wolfgang Amadé Mozart und seiner Familie in Verbindung stehen. Vollständig liegt bereits die Edition sämtlicher Mozart-Libretti vor. ${ }^{2}$ Als erste kritische Online-Edition der Texte der 25 musikdramatischen Werke Mozarts schließt sie eine wichtige Lücke in der Mozart-Philologie. Dabei wurde von jedem Werk der von Mozart vertonte Text und der des originalen, zur Uraufführung gedruckten Librettos, teilweise auch der literarischen Vorlagen ediert. Die Online-Edition der Mozart-Libretti nutzt die Möglichkeiten der digitalen Anzeige, beispielsweise indem neben dem edierten Text auch die Lesarten der Quellen parallel angezeigt werden können. Interdisziplinär ist dieses Projekt der DME aufgrund

1 Vgl. das interne Memorandum of Understanding zwischen PHI und der ISM.

2 Internationale Stiftung Mozarteum, Salzburg, Mozart-Libretti - Online-Edition, Salzburg 2007ff., online unter: https://dme. mozarteum.at/text-editions/librettos/ (11.03.2019); vgl. auch lacopo CIVIDINI, Von der literarischen Vorlage zum vertonten Text. Fünf Schritte zu einer digitalen textgenetischen Edition von Operntexten am Beispiel von Wolfgang Amadé Mozarts Die Entführung aus dem Serail KV 384, in: editio 31 (2017), 79-89. 
seiner Verbindung zwischen editorischen Interessen der Germanistik, Romanistik und Musikwissenschaft. Exemplarisch deutlich wird dies anhand der phonetisch-metrischen Analyse der von Mozart vertonten italienischen Libretti. Sie bietet ein dynamisches Instrumentarium, um die komplexe Beziehung zwischen metrischem Duktus der Textvorlage und dessen musikalischer Umsetzung näher erforschen zu können. Darüber hinaus befinden sich zwei weitere Projekte im Rahmen von DME::Librettos \& Texts in Vorbereitung: Das Projekt Nissen Online sieht eine kritische Edition der ersten umfangreichen Mozart-Biographie vor, die der dänische Diplomat und zweite Ehemann Constanze Mozarts, Georg Nikolaus Nissen, zusammengestellt hatte und die 1829 im Verlag Breitkopf \& Härtel erstmals erschienen war. ${ }^{3}$ Sie legt die bislang nur in Ansätzen bekannten, äußerst vielschichtigen Quellen offen, auf denen die Biographie beruht, kommentiert sie und verlinkt die diversen Primär- und Sekundärquellen mit digitalen Ressourcen. Ebenfalls wird derzeit das Projekt Leopold Mozart Violinschule - Digitale Edition vorbereitet. ${ }^{4}$ Vorgesehen ist anlässlich des 300. Geburtstags von Leopold Mozart im Jahr 2019 eine kritische digitale Edition von dessen Violinschule online vorzulegen. In einem ersten Schritt wird sie die ersten drei deutschen Auflagen des Lehrwerks umfassen; später sollen diese durch die historische niederländische und französische Übersetzung der Violinschule ergänzt werden.

Ebenfalls die Ansprüche einer volldigitalen Edition erfüllt das Projekt DME::Letters \& Documents mit einer Online-Publikation von Briefen der Mozart-Familie sowie von Dokumenten zur Familien- und Stiftungsgeschichte. ${ }^{5}$ So sind über dieses Projekt z.B. sämtliche Briefe Wolfgang Amadé Mozarts in einer diplomatischen Transkription verfügbar. Soweit dies rechtlich möglich ist, wird parallel auch ein Bild des Briefes angeboten, um Transparenz zwischen der Transkription und der Originalhandschrift herzustellen. Im Zentrum stehen die Bestände an Briefen und Dokumenten der ISM als weltweit größte Sammlung von Schriftstücken aus der Mozart-Familie, wobei die Briefe und Dokumente Wolfgang Amadé Mozarts und seines Vaters Leopold den überwiegenden Anteil ausmachen.

Als Kernprojekt der DME ist die Musikedition DME::Music zu bezeichnen. ${ }^{6}$ Dazu gehört zunächst das erste Projekt der DME: die bereits 2006 veröffentlichte NMA Online. Diese bietet Nutzer*innen die Möglichkeit, sämtliche 26.000 Seiten der gedruckten Bände der gesamten, über mehr als 50 Jahre erarbeiteten Neuen Mozart-Ausgabe (NMA) online als PDF-Scans abzurufen. Neben den Vorworten, Notenseiten und Anhängen der einzelnen Bände kann direkt auch auf die entsprechenden Kritischen Berichte zugegriffen werden. Außerdem wurden beispielsweise englischsprachige Vorworte ergänzt. Wenn in den Kritischen Berichten, die in vielen Fällen erst einige Zeit nach dem Notenband erschienen sind, für bestimmte Takte Berichtigungen oder Ergänzungen vermerkt wurden, lassen sich diese durch einen Link im Notentext unmittelbar aufrufen. Zusätzlich sind die einzelnen Werke mit den Aufnahmen der Mozart Ton- und Filmsammlung verknüpft, sodass Nutzer*innen sich einen Überblick über die vorhandenen Aufnahmen verschaffen können. Dabei stellt die NMA Online von jedem Mozart-Werk mindestens eine Einspielung zur Verfügung, die direkt über die Website abgerufen werden kann. So können Partiturverlauf und Einspielung parallel verfolgt werden. DME::Music bietet auf diese Weise eine weitere wichtige Zugangsebene für die Auseinandersetzung mit Mozarts Musik.

3 Internationale Stiftung Mozarteum, Salzburg, Nissen Online, Salzburg 2014ff., online unter: https://dme.mozarteum.at/ text-editions/nissen-online/ (11.03.2019).

4 Internationale Stiftung Mozarteum, Salzburg, Leopold Mozart Violinschule - Digitale Edition, Salzburg 2018ff., online unter: https://dme.mozarteum.at/text-editions/leopold-mozart-violinschule/ (11.03.2019).

5 Internationale Stiftung Mozarteum, Salzburg, Mozart Briefe und Dokumente - Online-Edition, Salzburg 2007ff., online unter: https://dme.mozarteum.at/briefe-dokumente/ (11.03.2019).

6 Internationale Stiftung Mozarteum, Salzburg, DME::Music, Salzburg 2014ff., online unter: https://dme.mozarteum.at/ musik/ (11.03.2019). 
Bei der NMA Online handelt es sich um eine bildbasierte Online-Präsentation, die also noch nicht, wie beschrieben, die Ansprüche einer genuin digitalen Edition erfüllt. Dieser erste digitale Schritt verfolgt primär das Ziel, den Zugriff auf die historisch-kritische Gesamtausgabe der Werke Wolfgang Amadé Mozarts zu vereinfachen und für Interessierte einen uneingeschränkten Zugang zu gewährleisten.

Als Nachfolgeprojekt der NMA Online versteht sich die Digital Interactive Mozart Edition (DIME) ${ }^{7}$, die auf den wissenschaftlichen Ergebnissen der NMA basiert, mit dieser aber nicht identisch ist und diese auch nicht ersetzt. DIME ist im Gegensatz zur NMA Online eine genuin digitale Edition, die mit dem XML-basierten Format der Music Encoding Initiative (MEI) arbeitet. Sie ermöglicht es, die Codierung mit weiteren Informationen anzureichern, die über den reinen Notentext hinausgehen, etwa zu editorischen Entscheidungen oder den Metadaten des entsprechenden Werkes. Hierdurch kann ein höherer Grad an Transparenz als bei gedruckten Editionen erreicht werden. Außerdem sind jederzeit Aktualisierungen möglich, die durch Vergabe von Versionsnummern nachvollzogen werden können. Dabei verfolgt DIME zwei editorische Stränge: Auf der einen Seite wird der gesamte Notentext der gedruckten NMA-Bände in sogenannten Referenztexten entsprechend in MEI codiert und angereichert. Die Basis hierfür bildet die Konvertierung des vollständigen Notentexts der NMA in ein MEl-konformes XML-File, das im Folgenden intensiv Korrektur gelesen, nach Richtlinien bearbeitet und um zusätzliche Informationen ergänzt wird, bevor die einzelnen Werke sukzessiv online geschaltet werden. Auf der anderen Seite werden ausgewählte Werke im Rahmen von sogenannten Alternativtexten neu ediert. Dabei handelt es sich um Quelleneditionen, die bereits vorhandene Referenztexte ergänzen.

Die entsprechend in MEl codierten Notentexte werden in einer eigens entwickelten Webanwendung, dem Digital Mozart score Viewer (MoVi), visualisiert, der wiederum auf dem durch RISM-Schweiz entwickelten Visualisierungstool Verovio fußt. ${ }^{8}$ Die diversen Features von MoVi werden ständig erweitert und fortentwickelt. In der folgenden Übersicht werden deshalb diejenigen Möglichkeiten der Benutzung und Werkzeuge vorgestellt, die beim First Release des MoVi am 14. Dezember 2018 implementiert waren (vgl. Abb. 1).

Unterhalb des Fensters, in dem der Notentext angezeigt wird, befinden sich verschiedene Schaltflächen, die der Navigation innerhalb des Notentextes dienen. So kann geblättert, durch Eingabe einer bestimmten Takt- oder Seitenzahl diese direkt angewählt und über eine Zoom-Leiste die Größe des angezeigten Notentextes verändert werden. Durch das Aktivieren der "Original-Zeilenumbrüche“ kann die dynamische Anzeige des Notentextes ausgeschaltet und der NMA angeglichen werden. Die letztgenannte Funktion ist insbesondere dann interessant, wenn ein Referenz- oder Alternativtext mit dem entspechenden Scan aus der NMA Online verglichen werden soll, was durch Klicken auf die Schaltfläche „Notentext in der NMA Online ansehen“ in der rechten Menüspalte unter „Übersicht“ im Splitscreen problemlos möglich ist. Ebenfalls unter dem Menüpunkt „Übersicht“ lassen sich diejenigen Werke, die bereits im Internet zur Verfügung stehen, über die „Werksuche“ nach KV-Nummern auswählen. Nach der Auswahl werden die einzelnen Sätze des Werks angezeigt. Außerdem kann hier falls vorhanden - zu dem entsprechenden Alternativtext, etwa der Quellenedition nach Autograph oder

7 Internationale Stiftung Mozarteum, Salzburg, Digital Interactive Mozart Edition, Salzburg 2018ff., online unter: https://dme. mozarteum.at/musik/edition/ (11.03.2019); vgl. auch Norbert DuBowY, Bereitstellung und Nutzung von Daten in der Digitalen Mozart Edition, in: Klaus Pietschmann, Hg., Symposiumsbericht „Wege des Faches - Wege der Forschung?“, Mainz 2018, 1-6, URN: nbn:de:101:1-2018122012143896934537.

8 RISM-Schweiz, Verovio. A music notation engraving library, [0. O. o. J.], online unter: https://www.verovio.org/index.xhtml (11.03.2019). 
Erstdruck, gewechselt werden. Mit dem Release wurden die sechs sogenannten Haydn-Quartette (KV 387, 421, 428, 458, 464 und 465), die Serenade in G Eine kleine Nachtmusik KV 525, das Ave verum corpus KV 618 und das Rondo der Vitellia "Non più di fiori“ aus La clemenza di Tito KV 621 als Referenztexte publiziert. Darüber hinaus wurden als Quelleneditionen das Streichquartett in B KV 458 vollständig nach dem Erstdruck und der erste Satz nach dem Autograph im Rahmen der Alternativtexte vorgelegt.

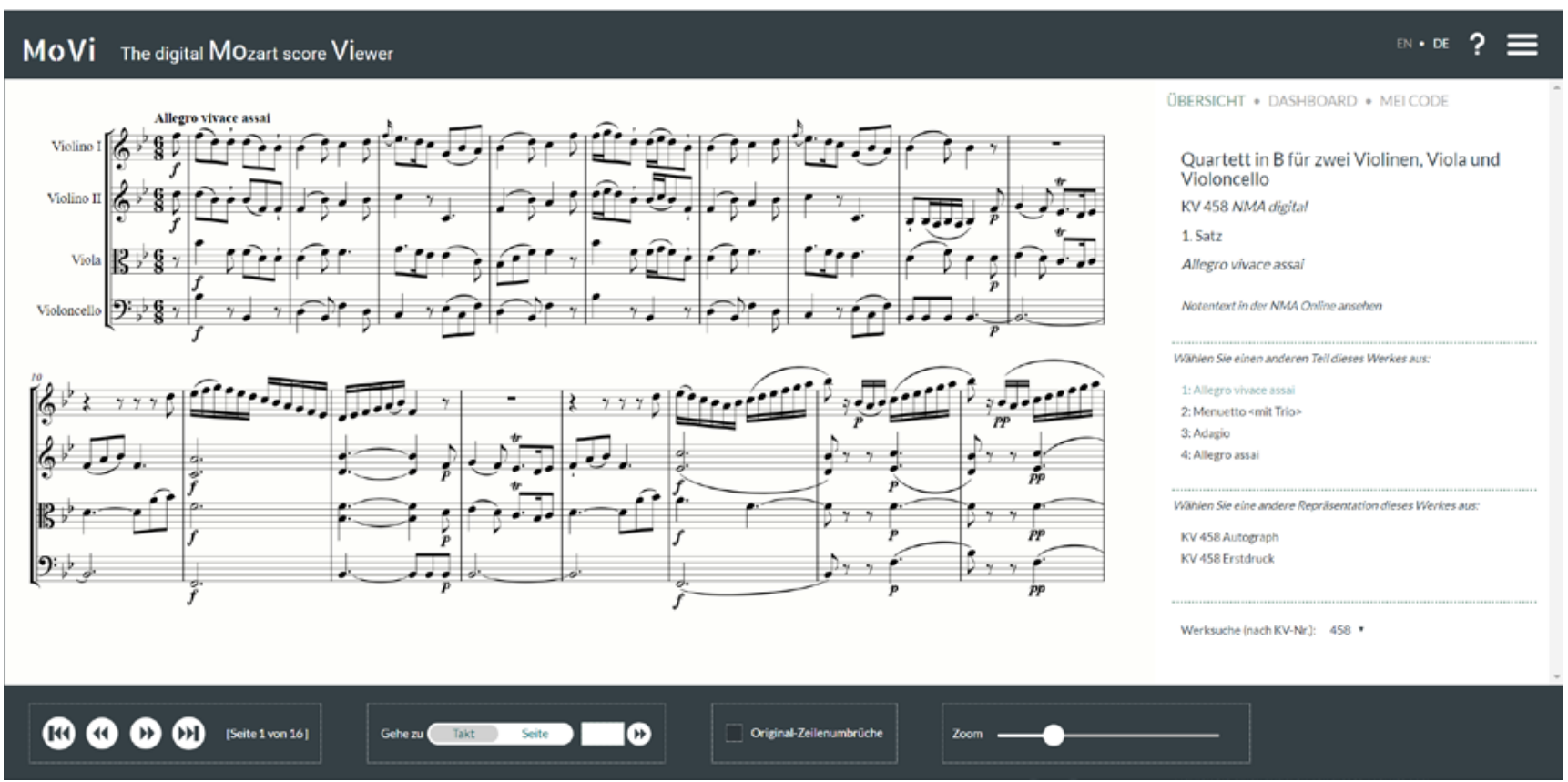

Abb. 1: MoVi, Übersicht zu KV 458, 1. Satz
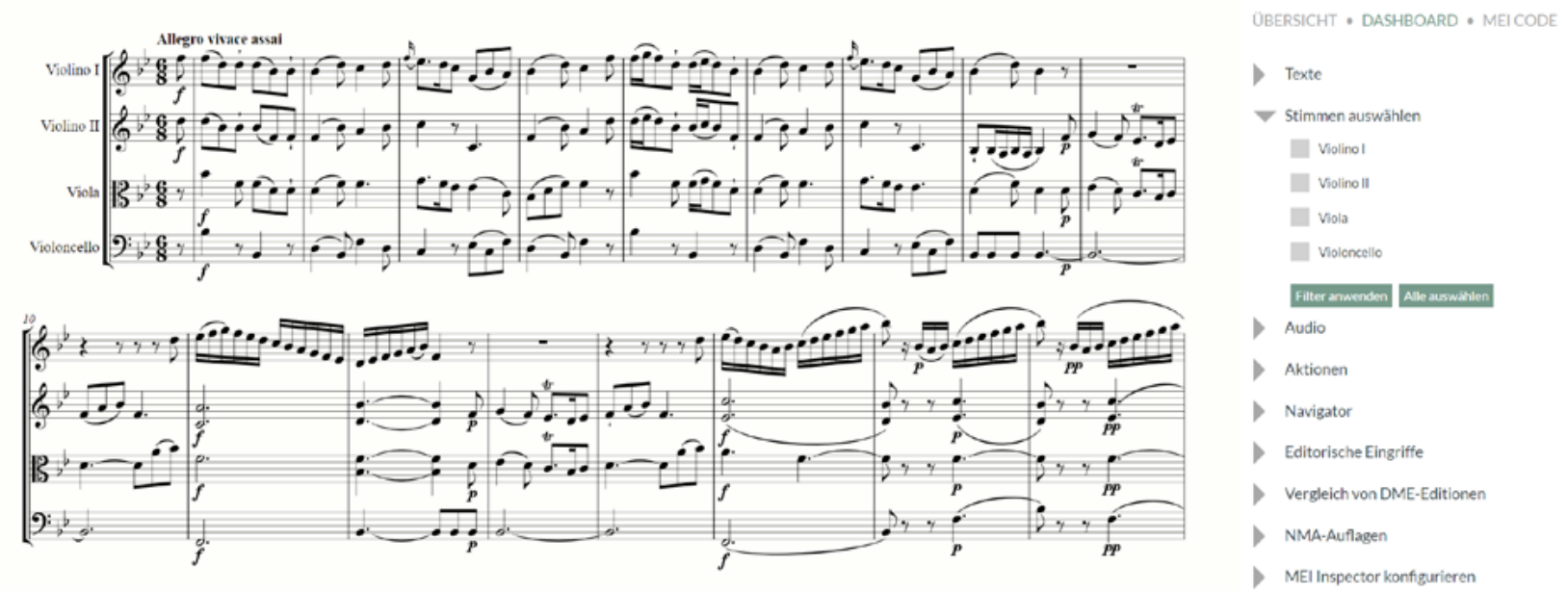

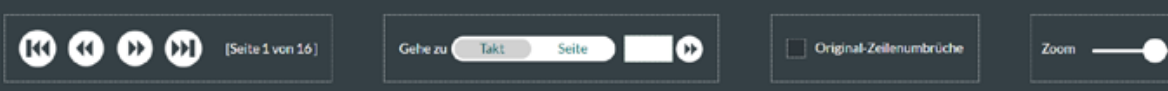

Abb. 2: MoVi, Dashboard

Wechselt man in der rechten Menüspalte von „Übersicht“ zu „Dashboard“, lassen sich verschiedene Werkzeuge auswählen. Hierunter finden sich praktische Tools, die etwa die Möglichkeit bieten, sich nur einzelne Stimmen anzeigen zu lassen, oder unter „Aktionen“ eine Funktion zum PDF-Druck des Noten- 
textes. Auch steht hier das gesamte File als Download sowie die Metadaten im Dublin Core-Format ${ }^{9}$ zur Verfügung. Der gesamte Webauftritt der DME steht unter der Lizenz CC BY-SA NC 4.010. Weitergabe und Bearbeitung der Files sind demnach unter bestimmten Bedingungen für nicht-kommerzielle Zwecke erlaubt. Der codierte Notentext kann im MIDI-Format abgespielt werden, für manche Werke steht auch eine Audio-Synchronisation mit mindestens einer Einspielung zur Verfügung. Unabhängig vom gewählten Abspielmodus wird der zu hörende Takt farblich hinterlegt, am Seitenumbruch blättert MoVi automatisch zur folgenden Seite um. Nutzer*innen können über den „MEl-Inspector“ auswählen, ob beim Klicken in den Notentext der MEI-Code eines einzelnen Elements, einer Stimme oder eines ganzen Taktes angezeigt werden soll. Der „Navigator“ dient der Zitation. Verwiesen werden kann mit inm auf ein gesamtes Werk, einen Satz daraus oder - noch detaillierter - auf einen bestimmten Takt bzw. eine Stimme in diesem Takt, indem eine URL erzeugt wird, mit der die ausgewählte Stelle präzise nachgewiesen werden kann. Philologisch untermauert wird DIME durch Texte zur Edition und Codierung, die einem Vorwort analoger Notenausgaben entsprechen. Bei Alternativtexten sind Quellenbeschreibungen und -bewertungen Teil des editorischen Angebots. Außerdem finden sich hier Schaltflächen, mit denen editorische Eingriffe im Notentext farblich hervorgehoben werden. Hier können gegebenenfalls auch Unterschiede zwischen NMA-Auflagen angezeigt oder die Differenzen zwischen den diversen Editionen von DIME (Referenztext vs. Alternativtext) visualisiert werden. Im Fall der Alternativtexte besteht außerdem die Möglichkeit, Annotationen der Editor*innen einzublenden. Augenfällig wird der Zusammenhang von Visualisierung und Codierung, wenn in der rechten Menü-Spalte „MEl Code“ ausgewählt wird: Dies hat zur Folge, dass der Code neben dem Notenbild sichtbar wird, wobei ein entweder im Code oder im Notentext angewähltes Element in den beiden Darstellungsformen jeweils hervorgehoben und somit eine Verbindung zwischen beiden geschaffen wird.

MoVi The digital Mozart score Viewer $? \equiv$
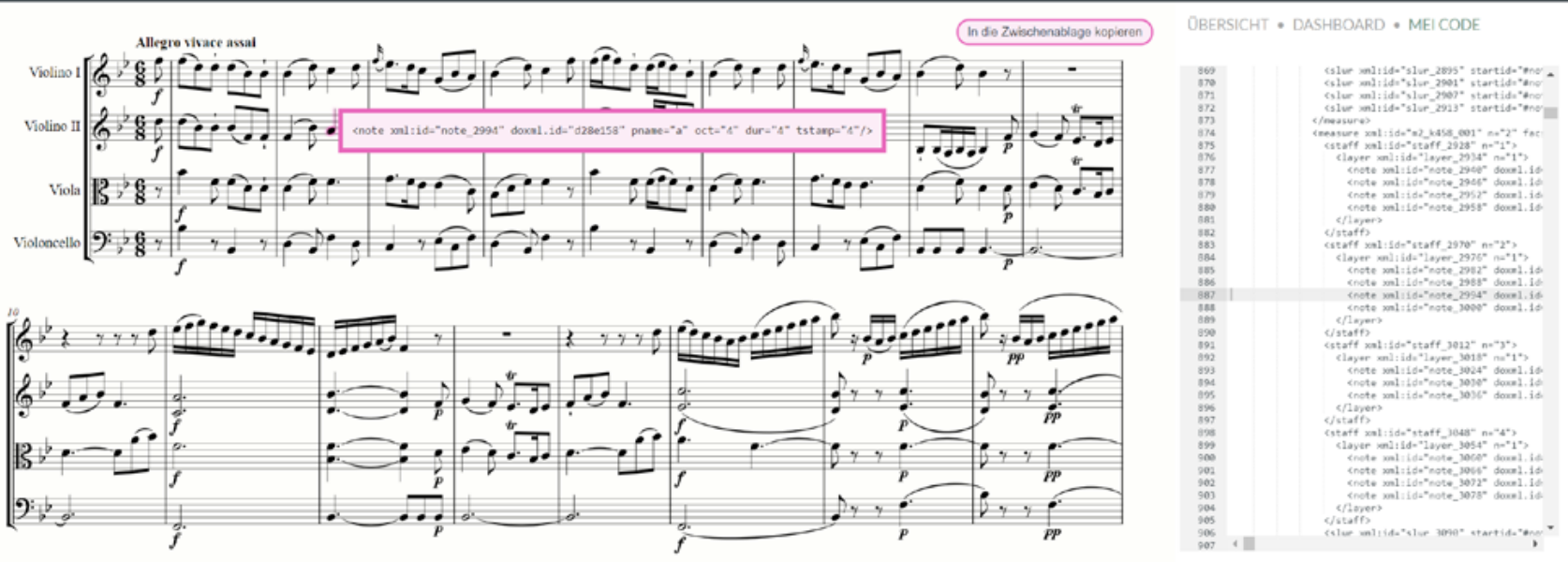

\section{$0000-1-0$}

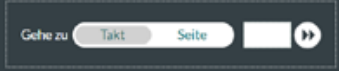

Orisinatzedenumbriche

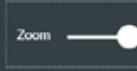

Abb. 3: MoVi, Verbindung Notentext - MEl Code

$9 \quad$ N. N., Dublin Core Metadata Initiative, [o. O.] 1995-2019, online unter: http://dublincore.org/ (11.03.2019).

10 Creative Commons, Namensnennung - Weitergabe unter gleichen Bedingungen 4.0 International (CC BY-SA 4.0), Mountain View, California (USA) [o. J.], online unter: https://creativecommons.org/licenses/by-sa/4.o/deed.de (11.03.2019). 
In Zukunft werden die Angebote der DIME regelmäßig erweitert und aktualisiert: Monatlich kommen im Bereich der Referenztexte neue Werke hinzu. Auch das Angebot der Alternativtexte soll stetig erweitert werden. Durchaus der Philosophie der DME entspricht es, dass Nutzer*innen die MEI-Codierung weiternutzen, indem sie die von DIME zur Verfügung gestellten Daten in eigene Projekte integrieren. Das daraus entstehende Potenzial scheint vielversprechend. Mit dem Umfang und Anspruch der diversen Projekte unter dem Dach der DME bricht die Mozart-Editorik in ein neues Zeitalter auf.

\section{Literaturverzeichnis}

lacopo CIVIDINI, Von der literarischen Vorlage zum vertonten Text. Fünf Schritte zu einer digitalen textgenetischen Edition von Operntexten am Beispiel von Wolfgang Amadé Mozarts Die Entführung aus dem Serail KV 384, in: editio 31 (2017), 79-89.

Creative Commons, Namensnennung - Weitergabe unter gleichen Bedingungen 4.0 International (CC BY-SA 4.0), Mountain View, California (USA) [0. J.], online unter: https://creativecommons.org/licenses/by-sa/4.0/ deed.de (11.03.2019).

Norbert DUBOWY, Bereitstellung und Nutzung von Daten in der Digitalen Mozart Edition, in: Klaus Pietschmann, Hg., Symposiumsbericht „Wege des Faches - Wege der Forschung?“, Mainz 2018, 1-6, URN: nbn:de:101:1-2018122012143896934537.

Internationale Stiftung Mozarteum, Salzburg, Digital Interactive Mozart Edition, Salzburg 2018ff., online unter: https://dme.mozarteum.at/musik/edition/ (11.03.2019).

Internationale Stiftung Mozarteum, Salzburg, Leopold Mozart Violinschule - Digitale Edition, Salzburg 2018ff., online unter: https://dme.mozarteum.at/text-editions/leopold-mozart-violinschule/ (11.03.2019).

Internationale Stiftung Mozarteum, Salzburg, Mozart Briefe und Dokumente - Online-Edition, Salzburg 2007ff., online unter: https://dme.mozarteum.at/briefe-dokumente/ (11.03.2019).

Internationale Stiftung Mozarteum, Salzburg, Mozart-Libretti - Online-Edition, Salzburg 2007ff., online unter: https://dme.mozarteum.at/text-editions/librettos/ (11.03.2019).

Internationale Stiftung Mozarteum, Salzburg, Nissen Online, Salzburg 2014ff., online unter: https://dme. mozarteum.at/text-editions/nissen-online/ (11.03.2019).

N. N., Dublin Core Metadata Initiative, [o. O.] 1995-2019, online unter: http://dublincore.org/ (11.03.2019).

RISM-Schweiz, Verovio. A music notation engraving library, [o. O., o. J.], online unter: https://www.verovio.org/ index.xhtml (11.03.2019). 


\title{
Saving Digital Humanities
}

\author{
„Langzeitarchivierung by Design“
}

Richard Hörmann \& Daniel Schlager

Universität Salzburg, IT Services

richard.hoermann@sbg.ac.at, daniel.schlager@sbg.ac.at

Keywords: Long-Term Archiving, IT-Infrastructure, Repository, Stefan Zweig digital, EbnerOnline, MHDBDB

\begin{abstract}
Archiving Digital Humanities projects without expiration dates is one of the biggest challenges which this young discipline faces. To meet this challenge, the University of Salzburg is building a platform on which digital containers ensure the safe storage of projects.
\end{abstract}

\section{Konzeption von dhPLUS (Richard Hörmann)}

Der Aufbau einer Plattform dhPLUS für die Langzeitarchivierung [LZA] von Digital Humanities [DH] Projekten an der Paris-Lodron-Universität-Salzburg [PLUS] geschieht im Rahmen eines 3-jährigen Projektes, das aus HRSM Strukturmitteln des österreichischen Wissenschaftsministeriums finanziert wird. Das Kompetenznetzwerk Digitale Edition [KONDE] genannte Projekt wird von der Universität Graz geleitet, die PLUS ist einer von mehreren österreichischen Partnern. Vom Rektorat der PLUS wurden die IT-Services der Universität Salzburg [ITS] beauftragt, das Projekt KONDE PLUS durchzuführen. Um die Plattform von Beginn an den Anforderungen der Zielgruppe entsprechend entwickeln zu können, wurden drei Pilotprojekte (Ferdinand Ebner: Gesammelte Werke. Online Edition [EbnerOnline] http://wfe.sbg.ac.at/, Mittelhochdeutsche Begriffsdatenbank [MHDBDB] http://mhdbdb. sbg.ac.at/, Stefan Zweig digital [SZD] http://www.stefanzweig.digital/) definiert, deren Migration auf die Plattform während der Projektlaufzeit vorgenommen wird. Dementsprechend setzt sich das Team aus Vertretern dieser Pilotprojekte und aus Mitarbeiter*innen der IT-Services zusammen, die auch den Leiter des gesamten Projektes stellen.

Das in der Planungsphase von dhPLUS entwickelte Konzept beinhaltet eine Reihe von SchlüsselParametern, die wie folgt formuliert wurden:

Schlüssel-Parameter der Plattform:

- Organisation: Die Plattform dhPLUS muss als ein Service der ITS der PLUS eingerichtet werden. Spätestens bei Start des Regelbetriebes müssen die ihr zugeordneten Stellen entfristet und aus dem Budget der ITS bzw. der PLUS finanziert werden (d.h. keine Drittmittel-Stellen). Die Plattform muss in die IT-Infrastruktur der ITS eingebunden werden, insbesondere an das vorhandene Datenbank- und Archivsystem. 
- Dokumentation: Die Plattform und ihre Projekte sind umfangreich zu dokumentieren. So wird gewährleistet, dass die trotz auftretenden und nicht prognostizierbaren Fluktuationen bezüglich Personal, Software etc. langfristig effizient bewältigt werden können.

- Daten-Sicherheit: Die Sicherheit der Daten ist in dreifacher Hinsicht zu garantieren: einmal in physikalisch-technischer mit „bitstream preservation“ Maßnahmen der lokalen IT-Infrastruktur. Zum zweiten bezüglich der Daten-Formate: Es sind standardisierte Formate zu verwenden, die die zukünftige Weiterverwendung, den Austausch und die Umstellung auf neue Formate ermöglichen. Und zum dritten bezüglich der Betriebssicherheit: Auch bei Ausfall von Teilen des Systems oder beim Austausch von Komponenten soll der Zugriff auf die Daten gewährleistet bleiben.

- Code-Sicherheit: Die in den DH-Projekten verwendete Software ist genauso zu sichern wie die Daten.

- Identifikation: Den digitalen Ressourcen der Plattform sind persistente Identifikatoren (PID) zuzuordnen, die eine eindeutige Identifikation der Ressourcen über ihre gesamte "Lebenszeit“ gewähren.

- Beschreibung: Die Daten der DH-Projekte auf der Plattform sind mit Metadaten zu ergänzen, die die Daten beschreiben und ihre Sicherung, Klassifikation, Weiterverarbeitung (Suche) und Wiederverwendbarkeit ermöglichen.

- Versionierung: Der Zugriff auf historische Versionen der Projekt-Ressourcen muss Teil der LZA-Strategie der Plattform sein.

- Bearbeitung: Die Plattform hat eine Archiv- und Bearbeitungsfunktion, d.h. sie hat nicht nur der Sicherung der Projektressourcen zu dienen, sondern genauso deren ständiger Bearbeitung, damit Ressourcen erweitert, korrigiert und optimiert werden können.

Bei der Erstellung von Modellen und Strategien zur Einhaltung und Umsetzung dieser Schlüsselparameter gab es zwei Leitideen: einerseits möglichst wenig dort zu erfinden, wo bereits Modelle oder Standards vorhanden sind und zum anderen dort innovativ zu sein, wo die bestehenden Lösungen unzureichend sind.

Ein Beispiel ist die Basis-Struktur der Plattform: obwohl von den Schlüssel-Parametern nicht vorgegeben, wurde auf das international anerkannte Open Archival Information System [OAIS]-Referenzmodell für offene Archivsysteme zurückgegriffen: 


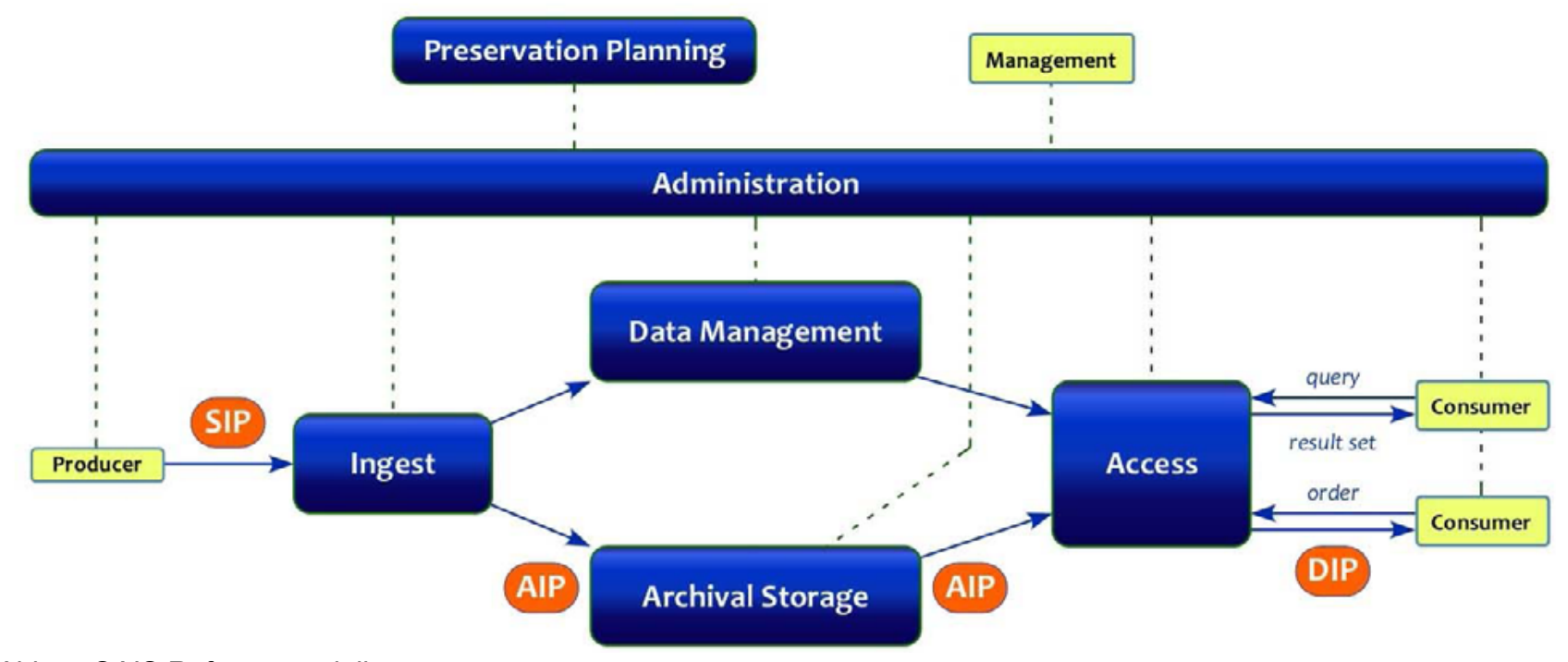

Abb. 1: OAIS Referenzmodell

Dem OAIS nach hängt eine erfolgreiche Archivierung digitaler Ressourcen davon ab, ob im System Informationspakete strukturiert vorliegen (Archival Information Package [AIP], Submission Information Package [SIP], Dissemination Information Package [DIP]), bestimmte Funktionen implementiert sind (Ingest, Archival Storage, Data Management, Access, Administration, Preservation Planning) und Rollen klar definiert sind (Producer, Management, Consumer).

Im OAIS Modell sind es keine Daten oder Ressourcen, die eingegeben, archiviert oder ausgegeben werden, sondern Informationen. Es gibt zwei Arten von Informationen, die in Informationspaketen [IP] abgelegt werden:

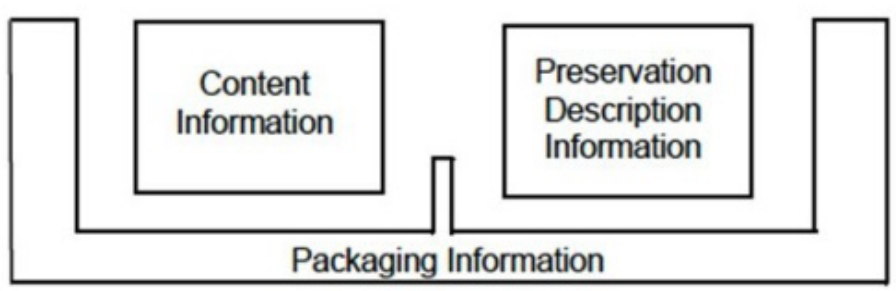

Abb. 2: OAIS Modell

1. Inhaltliche Informationen, in den digitalen Ressourcen repräsentiert als Projektdaten, die erhalten werden sollen: Content Information.

2. Informationen, die zur Erhaltung der inhaltlichen Informationen erforderlich sind: Preservation Description Information [PDI].

SIP, AIP und DIP sind Typen solcher IP, die sich bezüglich ihrer Position im Workflow des Systems unterscheiden:

- SIP sind Pakete, die vom Producer im Ingest an das System übergeben werden.

- AIP sind Pakete, die im System gespeichert und verwaltet werden.

- DIP sind Pakete, die vom Consumer aus dem System entnommen werden. 
OAIS ist ein Modell, in dessen Zentrum die Archivierung von Informationen steht. Da die Plattform dhPLUS aber für spezielle Projekte, nämlich Projekte der $\mathrm{DH}$, gebaut wird, ist es sinnvoll zu untersuchen, ob sich aus der Perspektive dieser Art von Projekten zusätzliche Anforderungen ergeben.

Obwohl DH-Projekte unter sich sehr heterogen sind und aufgrund der unterschiedlichen Projektziele, -methoden, technischen Realisierungen etc. auf allen Ebenen Singularitäten aufweisen, lassen sich doch aus den meisten Projekten grundlegende Gemeinsamkeiten abstrahieren.

Ein Modell, das diese Gemeinsamkeiten gut abbildet, ist das von der Berlin-Brandenburgischen Akademie der Wissenschaften [BBAW] vorgestellte Layer-Modell für Digitale Editionen:

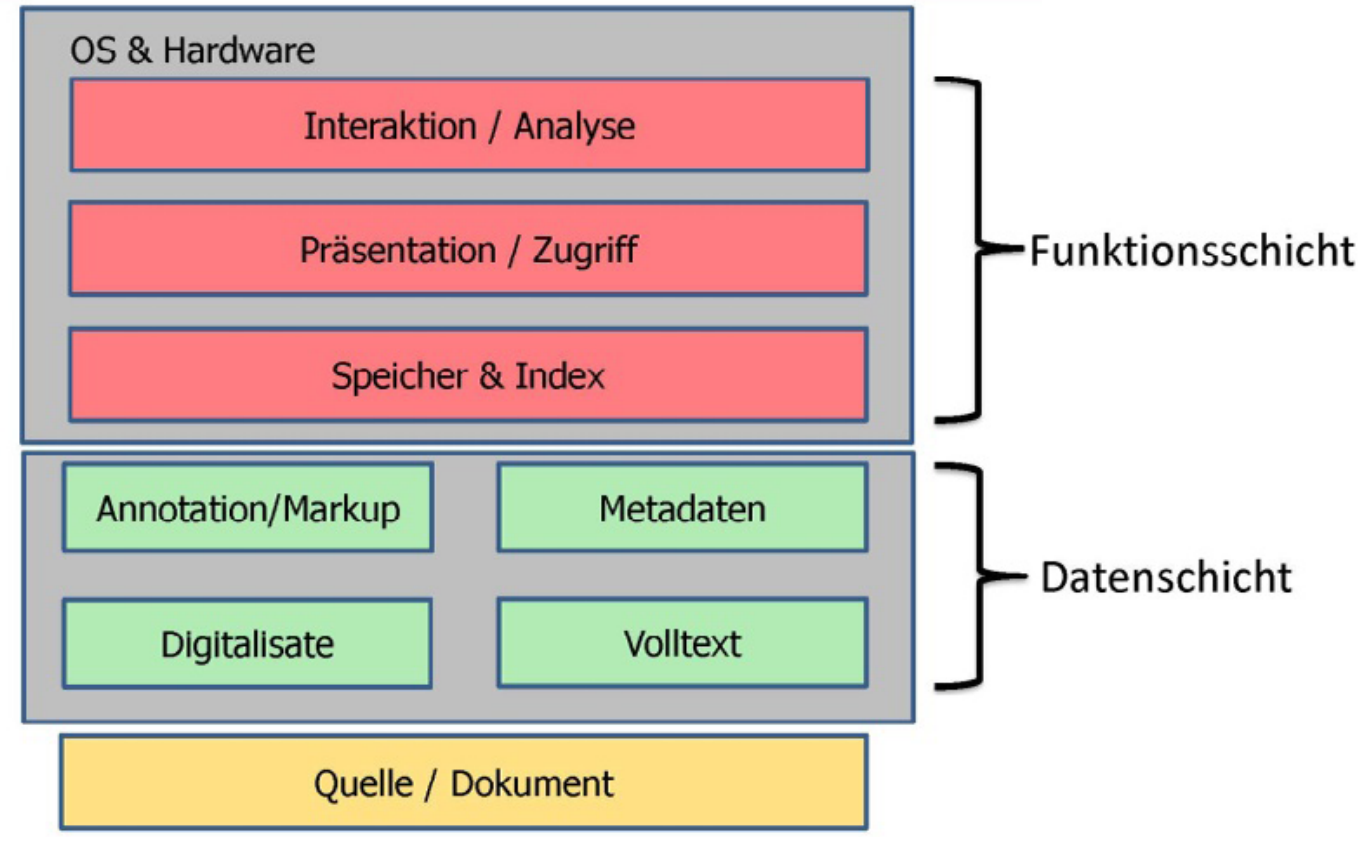

Abb. 3: Layer-Modell für Digitale Editionen

Diesem Modell zufolge besteht ein DH-Projekt aus zwei basalen Schichten: der Daten- und der Funktionsschicht. Sollen die Ergebnisse von DH-Projekten ohne Ablaufdatum gesichert werden, sind demnach nicht nur ihre Daten, sondern auch ihre Funktionen zu sichern.

Ein Schlüssel-Parameter für die LZA ist das Vorliegen einer auf dem aktuellen Stand gehaltenen, umfangreichen Dokumentation sowohl der Plattform als auch der einzelnen Projekte auf ihr. Da dieser Aspekt der LZA im Layer Modell der BBAW nicht berücksichtigt ist, ist das Layer Modell von dhPLUS um eine Dokumentations-Schicht zu ergänzen.

Aus dem erweiterten Layer-Modell folgt auch ein erweitertes OAIS-Modell für dhPLUS: zu den inhaltlichen Informationen der IP zählen jetzt neben den Informationen der Datenschicht auch die Informationen aus der Funktions- und Dokumentationsschicht.

OAIS- und Layer-Modell von dhPLUS sind abstrakte Modelle, die ein vermittelndes Konzept erfordern, das ihre SW-seitige Realisierung ermöglicht. Aus dem pragmatischen Ansatz, möglichst viel von bestehenden Lösungen zu übernehmen, wurde für dhPLUS ein ObjektModell ausgewählt, das im Geisteswissenschaftlichen Asset Management System [GAMS] der Universität Graz verwendet wird und seinerseits auf einem Fedora-Modell basiert. Das daraus für dhPLUS modellierte Digitale Objekt [DO] ist so aufgebaut: 


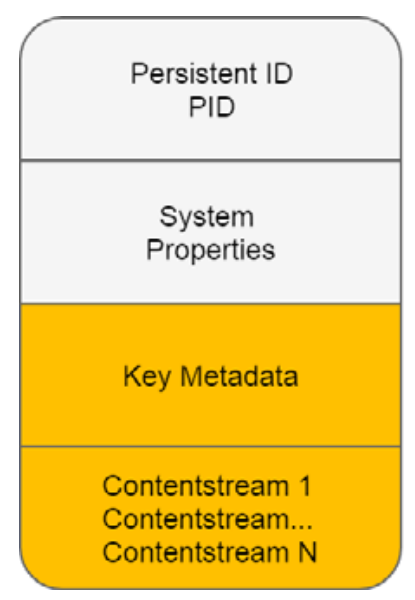

Abb. 4: Digitales dhPLUS-Objekt

Ein DO besteht aus vier Teilen: einem Teil mit dem eindeutigen Identifier des Objektes, einem zweiten mit den Systemeigenschaften des Digitalen Objektes, einem dritten mit den Schlüssel-Metadaten, und einem vierten mit den zu sichernden Inhalten des Objektes.

Systemeigenschaften und Schlüssel-Metadaten des DO unterscheiden sich insofern als erstere Metadaten sind, die Informationen über das DO in Bezug auf das Plattform-System beinhalten, während letztere Informationen über die Inhalte des DO (= Contentstreams) bereitstellen.

Dieses Modell eines DO erfüllt sowohl die Schlüsselparameter als auch die Anforderungen, die sich aus dem erweiterten OAIS und Layer-Modell von dhPLUS ergeben.

Die drei Schichten des Layer-Modells führen zu drei verschiedenen Typen von DO, die sich in Bezug auf die Contentstreams unterscheiden:

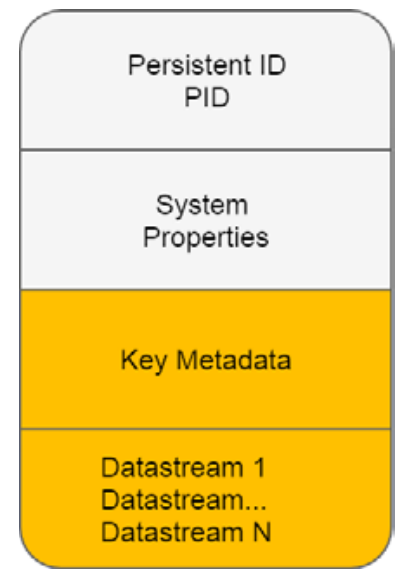

Abb. 5: Digitales Daten Objekt

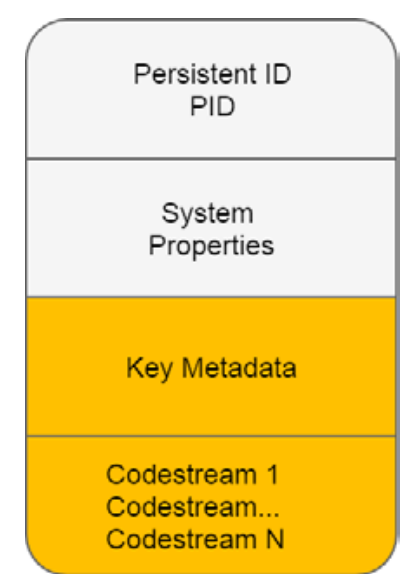

Abb. 6: Digitales Funktions Objekt

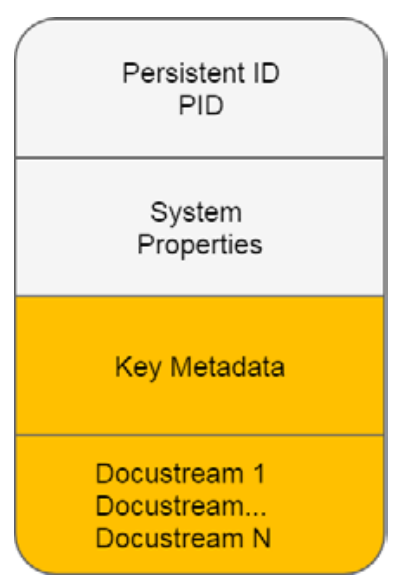

Abb. 7: Digitales Dokumentations Objekt

Ein Digitales Daten Objekt [DDO] enthält als Contentstreams Datastreams mit den Daten und Metadaten der Datenschicht, ein Digitales Funktions Objekt [DFO] enthält Codestreams mit den Funktionen und Services der Funktionsschicht und ein Digitales Dokumentations Objekt [DDCO] Docustreams, deren Contentstreams der Dokumentationsschicht entnommen sind.

Die in jedem IP des OAIS-Modells enthaltene PDI wird in den Key-Metadaten des DO modelliert. Die systemrelevanten Informationen für die Archivierung, das Datenmanagement und die Administration werden in den System Properties abgelegt. 
Mit der Persistent ID jedes Objektes ist der Schlüssel-Parameter der eindeutigen Identifikation jeder Projekt-Ressource gegeben.

\section{Technische Realisierung von dhPLUS (Daniel Schlager)}

Die persistente ID entspricht dem Universally Unique Identifier [UUID] Standard der Version 5. Bei dieser namensbasierten UUID wird aus einem DNS Namen eine UUID gebildet. Der Vorteil gegenüber der (Pseudo-)zufällig generierten UUID (Version 4) ist die garantierte Eindeutigkeit durch den einberechneten DNS Namen des Repositoriums und der Projekt-ID.

Die Projekt-ID entspricht einem Namenskürzel für ein spezifisches Projekt. Als Beispiel kann hier das Kürzel: MHDBDB angeführt werden.

Daraus errechnet sich folgende UUID: e6921bb7-212f-5922-9a62-f6e24f06669a

Aus dieser UUID wird eine URI erzeugt, die eine Ressource eindeutig identifiziert:

https://dhplus.sbg.ac.at/rest/MHDBDB/e6921bb7-212f-5922-9a62-f6e24f06669a

Ressourcen im dhPLUS Repository werden in RDF modelliert. Als Basisformat wird RDF/XML verwendet. Die XML Dokumente werden zur automatischen Verarbeitung in einer Oracle Datenbank gespeichert (und auch dort archiviert). Der Einsatz von Oracle als Datenbank und Archivierungssystem wurde aus strategischen Gründen gewählt. Grundsätzlich ist die dhPLUS Software modular und unabhängig von spezifischen Datenbanksystemen aufgebaut. Die Oracle Plattform, bestehend aus der Oracle Datenbank und dem Oracle Archivsystem, sind das Kernstück der IT Services der Universität Salzburg. Dies ermöglicht dhPLUS die einfache, kosteneffektive und sichere Implementierung und Umsetzung einer LZA-Strategie.

Durch den konsequenten Einsatz von RDF - nicht nur zur Modellierung der digitalen Objekte, sondern auch für die Projektkonfigurationen und andere Daten - sprechen wir bei dhPLUS von einem RDF bzw. Knowledge Graph basierten Repository.

Die RDF/XML Dokumente werden vom System in einem Triple Store, wofür aktuell Apache Jena eingesetzt wird, weiterverarbeitet und mittels einer REST-API und einem SPARQL-Endpoint zur Verfügung gestellt. Durch die Modularität und Aufteilung der API in Microservices ist ein Austausch des Triple Stores jederzeit möglich. 


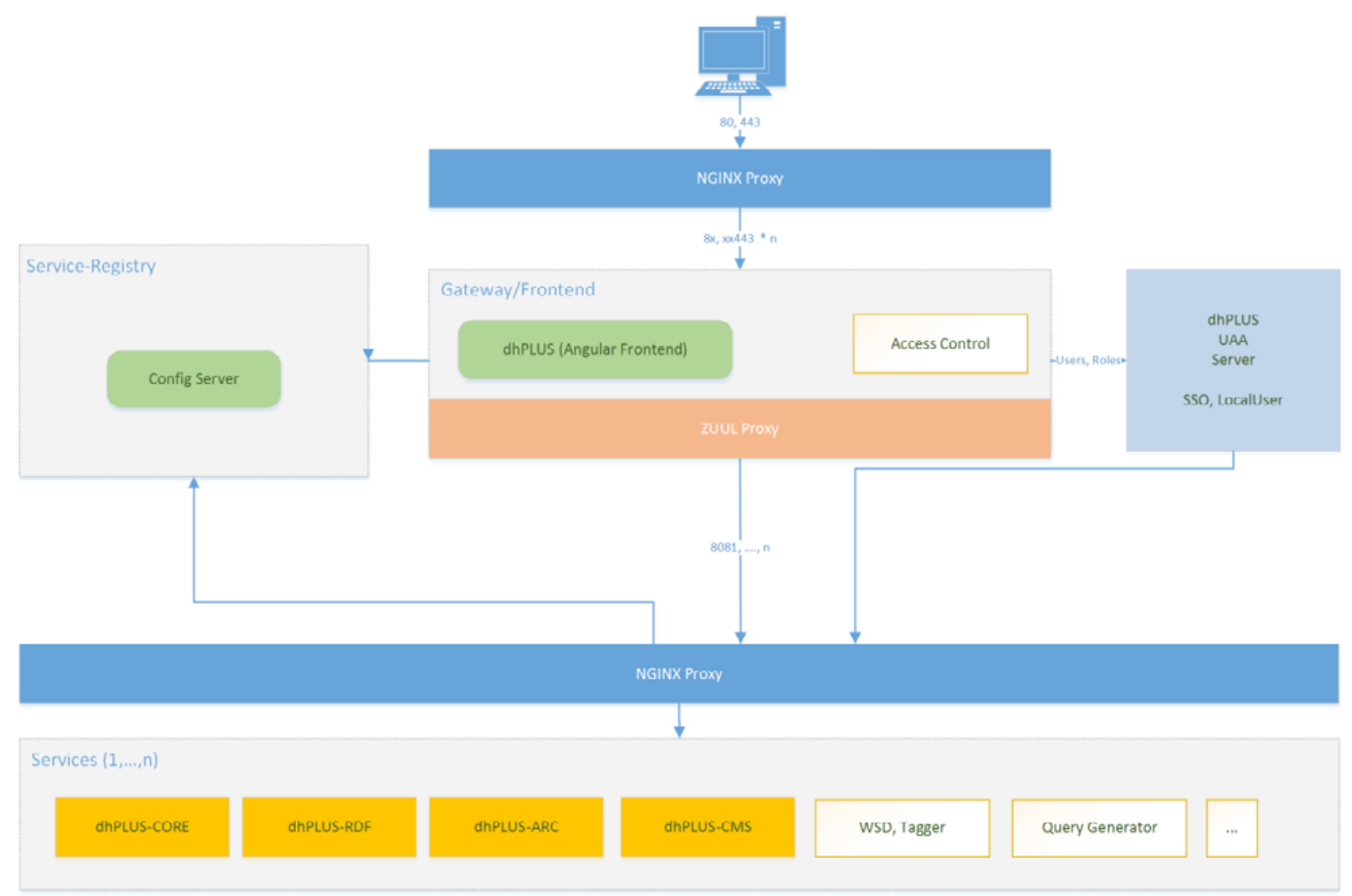

Abb. 8: Microservice Architektur dhPLUS

Ein weiterer Vorteil von Microservices gegenüber einem monolithischen Ansatz ist die leichtere Skalierbarkeit und dahingehend auch die hohe Verfügbarkeit.

In Abb. 8 ist zu sehen, dass jeglicher Zugriff auf einen Service und auf das vom Frontend zur Verfügung gestellte Gateway über einen Proxy gehandelt und verteilt wird. Zusätzlich zu diesem NGINX Proxy beinhaltet der Gateway Dienst einen eigenen Proxy (ZUUL), welcher die ankommenden API Requests an den zuständigen, in der Service-Registry registrierten Service weiterleitet.

Die API, welche die grundlegenden RDF Funktionalitäten für die Plattform bereitstellt, ist in einem eigenen Microservice (dhPLUS-RDF) verankert und nahezu 100\% kompatibel zu Fedora 4. Mit dieser Emulationsschicht kann ein Austausch mit anderen auf Fedora basierten Repositorien gewährleistet werden. 


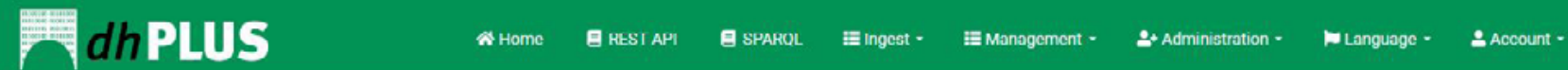

Digital Object

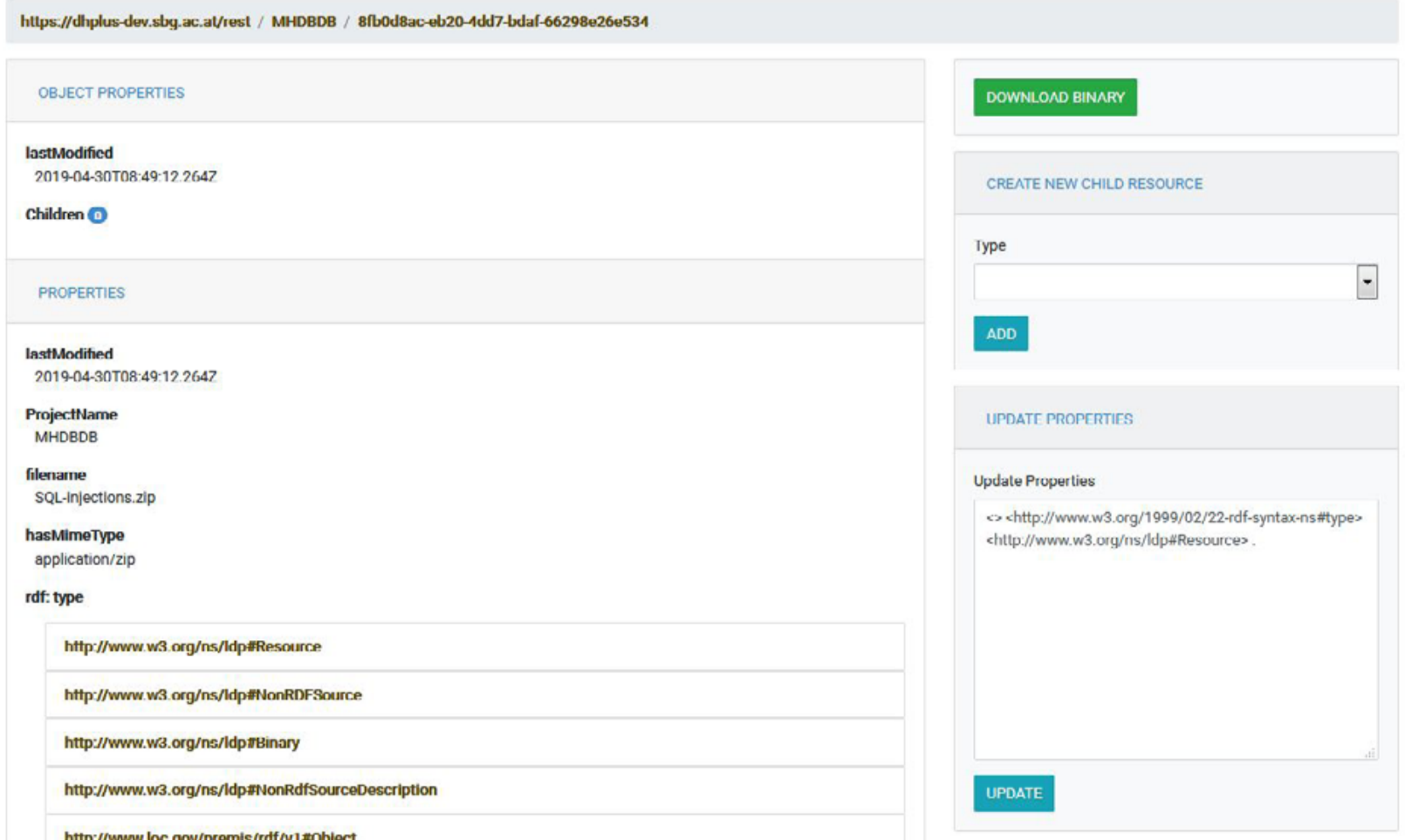

Abb. 9: REST-API dhPLUS

Abb. 9 zeigt das Beispiel eines Digitalen Daten Objektes [DDO] so wie es sich beim gegenwärtigen Stand von KONDE PLUS als Antwort auf einen REST-API Request im Browser darstellt. Zu sehen ist die PID des Beispiel-DDO (https://dhplus-dev.sbg.ac.at/rest/MHDBDB/8fb0d8ac-eb20-4dd7bdaf-66298e26e534), seine Object (= System) Properties und die Properties (= Key Metadata) der

Datastreams. Wie am Dateinamen ersichtlich handelt es sich bei den Datastreams um mehrere gezippte Dateien, die über den Button „Download Binary“ geöffnet werden können.

\section{Literaturverzeichnis}

Consultative Committee for Space Data Systems [CCSDS], Hg., Reference Model for an Open Archival Information System (OAIS), Washington D. C. 2012, 2-6, online unter: https://public.ccsds.org/pubs/650x0m2.pdf (13.05.2019).

Alexander CzmieL, Dokumentation, Werkzeugkasten, Pakete. Nachhaltigkeit von Daten und Funktionalität Digitaler Editionen, in: Konferenzabstracts. DHd2017 Bern. Digitale Nachhaltigkeit, Bern 2017, 138-141, online unter: http://www.dhd2017.ch/wp-content/uploads/2017/02/Abstractband_ergaenzt.pdf (13.05.2017).

OAIS-Based Processes, online unter: https://www.icpsr.umich.edu/icpsrweb/content/datamanagement/lifecycle/ oais.html (07.06.2019.)

Elisabeth Steiner / Johannes StIGLeR, GAMS and Cirilo Client. Policies, documentation and tutorial, Graz 2014 (Letzte Aktualisierung 10.04.2017), online unter: hdl.handle.net/11471/521.1.1 (13.05.2019). 


\title{
Feature analysis of paintings using color informa- tion of the image
}

\author{
Ryuhei Yukimura [1], Hao Sun [2] \& Mingzhe Jin [2] \\ [1] Ferry Sunflower Limited, Oita, Japan \\ [2] Culture and Information Science, Doshisha University, Kyoto, Japan \\ sapanyman10-job@yahoo.co.jp (Ryuhei Yukimura), sonnkou1985@gmail.com (Hao Sun), \\ mjin@mail.doshisha.ac.jp (Mingzhe Jin) \\ https://www1.doshisha.ac.jp/ mjin/
}

Keywords: Paintings, Color Information, Baroque Era, Feature Analysis, Image

\begin{abstract}
In this article, we quantitatively analyzed paintings drawn by three painters of the Baroque era. RGB and color code is used as features. Our results indicate that color code is superior to RGB in capturing the characteristics of paintings.
\end{abstract}

\section{Introduction}

Past research on paintings has been based on human knowledge and subjective perception. Research based on quantitative measures is underway due to advancements in digitization. RGB is the typical color information used to study images. For example, Takahashi, Sakamoto, and Kato captured color features of interior brands by analyzing the color information (converting RGB to $L^{*} u^{*} v^{*}$ ) of the corresponding images. Kashiwagi and Ooe examined a method to detect non-uniform color areas (color characteristic of objects) by analyzing the RGB data of images. However, RGB struggles to capture color features. It also represents colors of a given image by dividing it into three channels. This research focuses on the color code to capture color features in images. The color code is used to express colors on the Web or computers. A code is allocated to each color.

The purpose of this research is two-fold. The first is to capture the chromatic characteristics and styles of paintings by using the color information in images of them, and the second is to explore the effectiveness of color code data for the analysis of paintings. We quantitatively analyzed 130 paintings using the color code and RGB to capture their color features in images. We also compared the results to explore the effectiveness of the color code for the analysis of paintings. 


\section{Method}

The proposed method consists of three steps: First, paintings are scanned and converted into images. The paintings used in this research were from the same publisher. Second, we collect color code and RGB data from the images. Third, we analyze the data and draw conclusions from the results.

\subsection{RGB and color code data}

RGB data was collected from an RGB histogram which shows the degrees of brightness of the target color channel. To collect RGB data, gradations for each color of the RGB histogram were divided into 72 equal channels. The collected data was then converted into relative frequency. Data concerning the color code was collected by using Web-safe color images. We used full-color images that can represent a maximum of 16,777,216 colors, whereas Web-safe color images can be represented by a maximum of 216 colors. To make the images easier to handle we collected data concerning the color code as unigrams and bigrams. Bigrams consisted of the aggregation of both horizontal and vertical color code data.

\subsection{Target of analysis}

We analyzed three painters from the Baroque era: Michelangelo Merisi da Caravaggio (1571-1610), Rembrandt Harmenszoon van Rijn (1606-1669), and Johannes Vermeer (1632-1675). A total of 48 paintings by Caravaggio, 47 by Rembrandt, and 35 by Vermeer were analyzed. The Baroque era is a style of art, music, and architecture that flourished in Europe from the early 17th to the mid-18th century. During this era, the arts focused on dynamic expression intended for sensory effects, such as dramatic spatial expressions. Therefore, paintings by the three painters are characterized by thorough graphical depiction and strong contrast.

\subsection{Method of analysis}

The authors used multivariate analysis: specifically, principal component analysis (PCA) based on a matrix of correlation coefficients. When the dataset is configured using many variables, PCA explains the character of each sample using few variables. We can capture the color features of each painting through this analysis. We focus on the first and second principal components in PCA. 


\section{Results}

\subsection{Analysis using RGB data}

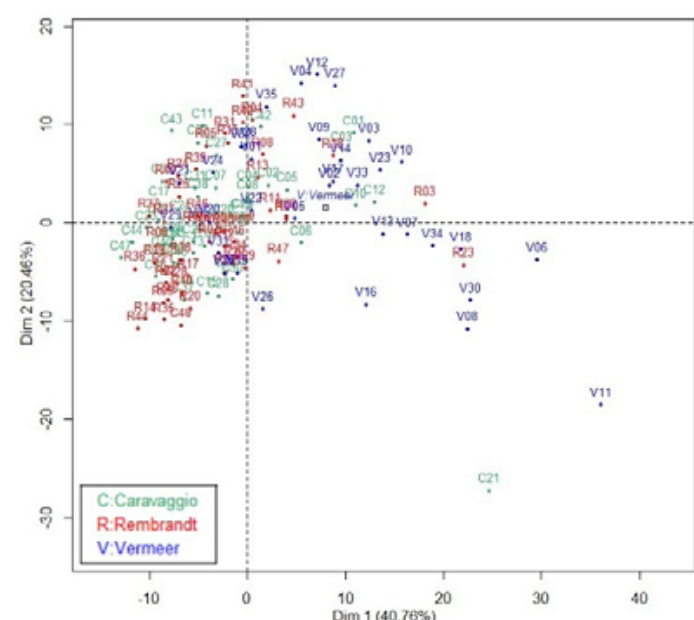

Fig. 1: Principal component loading (RGB)

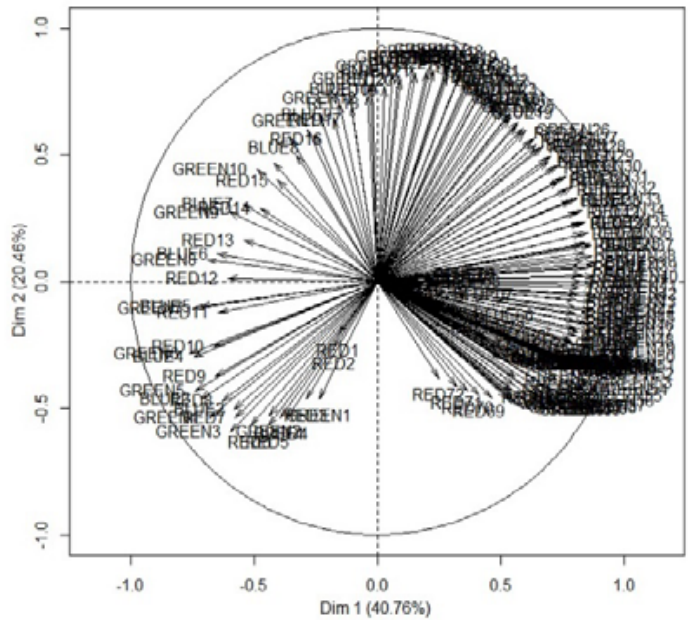

Fig. 2: Principal components pc1 and pc2 (RGB)

The first result obtained was that for PCA with three RGB channels. Figure 1 shows a plot of principal component loading, and Figure 2 shows the biplot of the first and second principal components.

Figure 1 shows bright colors when the number increases. Variables representing dark colors, such as RED1, RED2, and GREEN1, are in the lower-left part of Figure 1. Bright color variables increase along the clockwise direction. Paintings by Caravaggio and Rembrandt are located to the left in the figure, and Vermeer's paintings are located to the right in Figure 2. The result shows that dark colors are often used in Caravaggio's and Rembrandt's paintings, and bright colors are often used in Vermeer's paintings.

\subsection{Analysis using color code data}

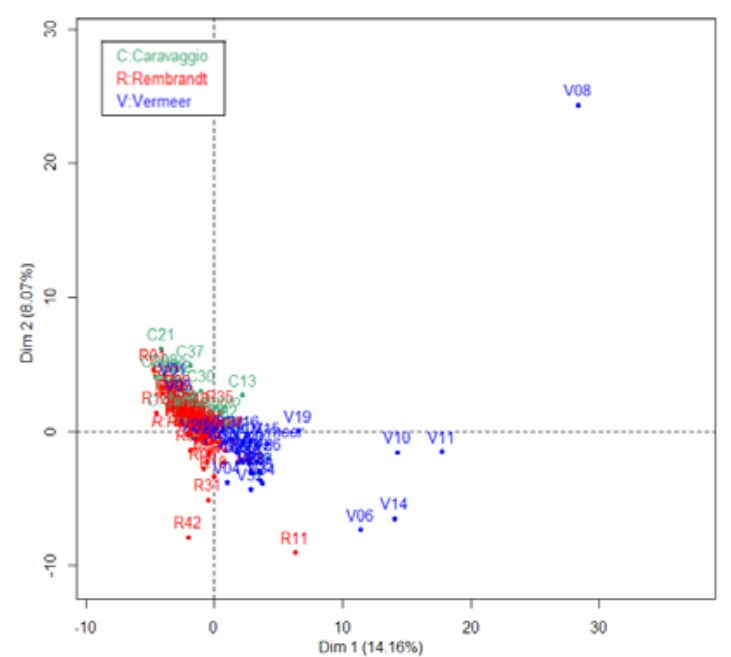

Fig. 3: Principal component loading (unigram, 130 works)

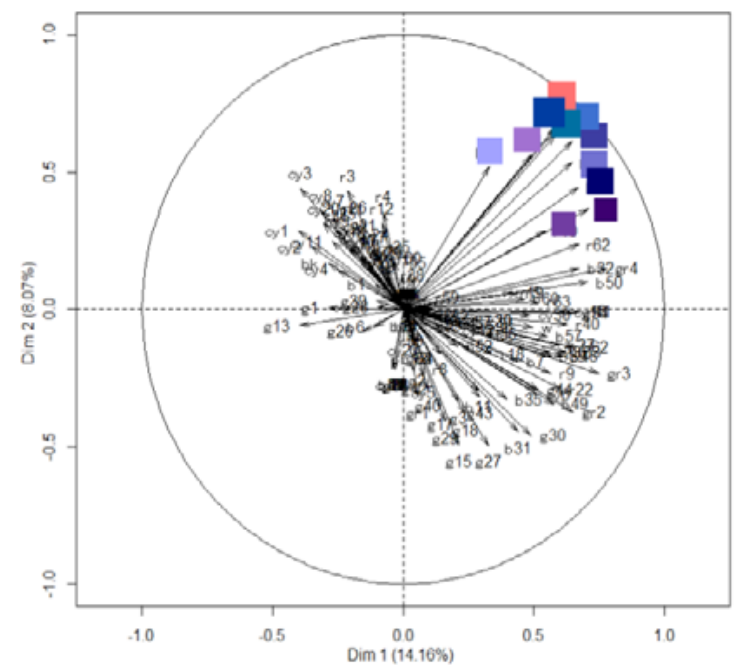

Fig. 4: Principal components pc1 and pc2 (unigram, 130 works) 
Figures 3 and 4 show the principal component analysis of 130 paintings using unigram data. Figure 3 shows a plot of principal component loading, and Figure 4 shows plots of the first and second principal components. Two paintings are out of position in Figure 4 and have color-specific characteristics. Dark red and dark blue are located in the upper-right corner of Figure 3. Moreover, the light green and light blue are in the bottom-right of Figure 3. However, Vermeer's painting no. 2 is in the upper-right of Figure 4. Therefore, it is unique in its use of dark red and dark blue. Rembrandt's painting no. 23 is in the bottom-right of Figure 4 and is thus unique in its use of light green and light blue.

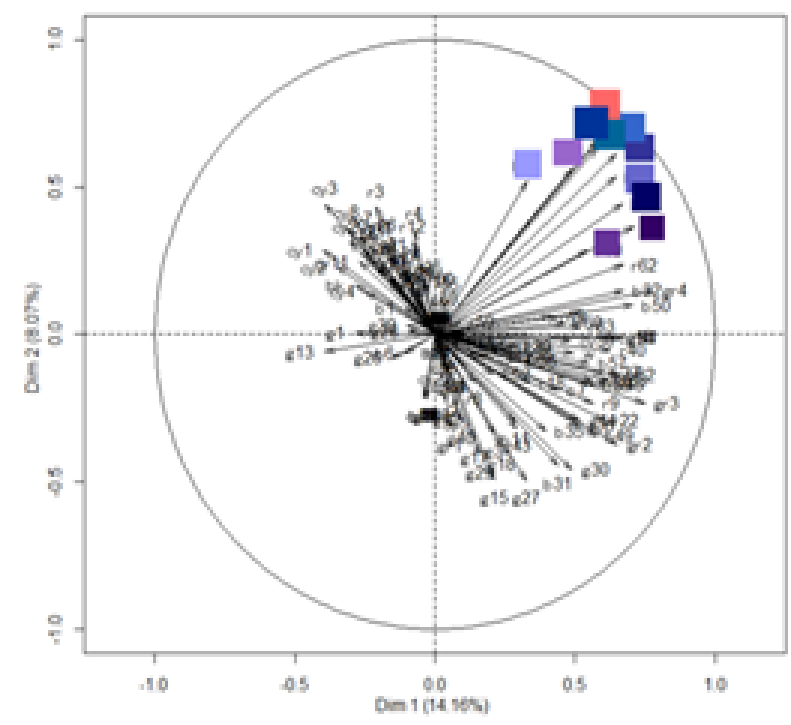

Fig. 5: Principal component loading (unigram, 128 works)

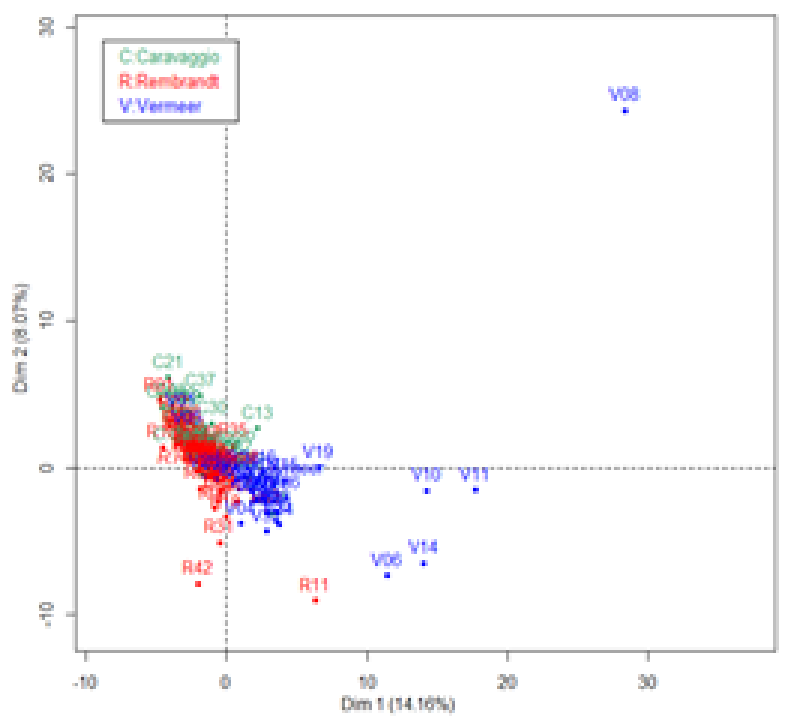

Fig. 6: Principal component loading (unigram, 128 works)

Figures 5 and 6 show results of the principal component analysis of 128 paintings using unigram data. We excluded two outliers, Vermeer's painting no. 2 and Rembrandt's painting no. 23. Figure 5 shows the plot of principal component loading and Figure 6 shows the biplot of the PCA. Vermeer's painting no. 6 has color-specific characteristics because in Figure 5 it is far from the other paintings. Moreover, because dark blue and vermilion are in the upper-right of Figure 6 Vermeer's painting no. 6 is unique in its use of dark blue and vermilion.

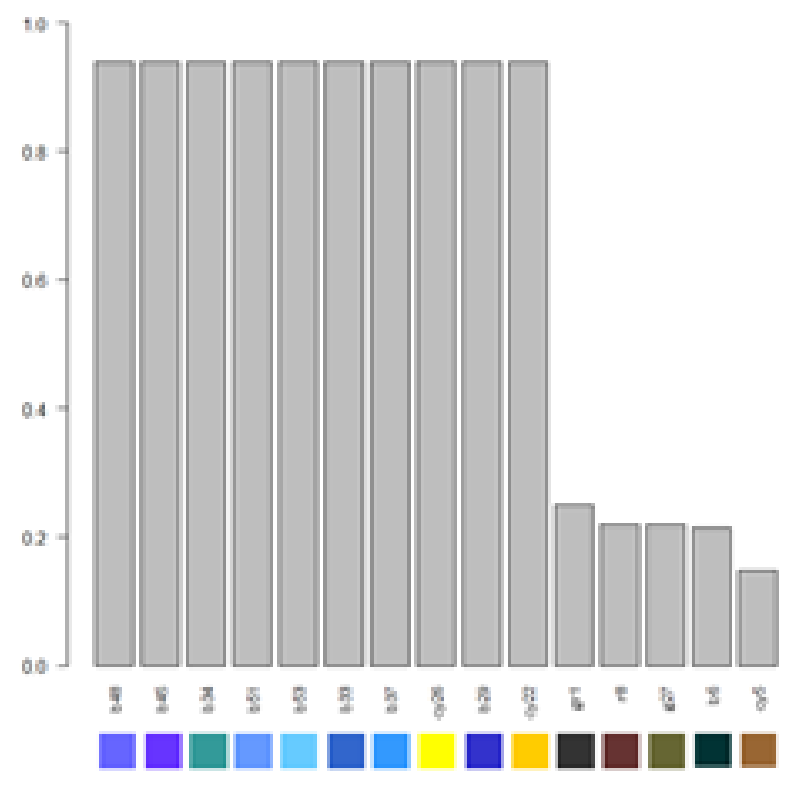

Fig. 7: Principal comp. loading of PC2 (unigram, 127 works, top10)

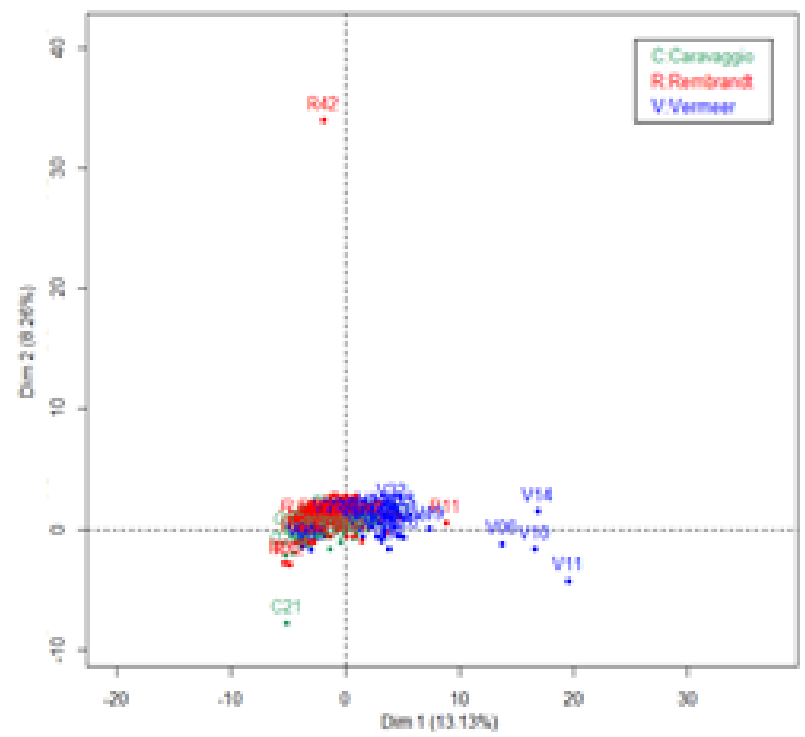

Fig. 8: Principal comp. loading (unigram, 127 works) 
Figures 7 and 8 show results of the principal component analysis of 127 paintings using unigram data. Vermeer's painting nos. 2 and 6, and painting no. 23 by Rembrandt were excluded for this analysis. Figure 7 shows a bar graph of the second principal component and Figure 8 shows the biplot of the principal component analysis. Vermeer's painting no. 6 has color-specific characteristics because its position is different from those of the other paintings. Blue and yellow are higher in rank in terms of the second principal component (Fig. 7). Therefore, Vermeer's painting no. 42 is unique in its use of blue and yellow.

Figures 9 and 10 show the principal component analysis of 126 paintings using unigram data. The four unique paintings described earlier were excluded. Figure 9 shows a plot of principal component loading and Figure 10 shows plots of the first and second principal components. Warm colors are to the left in Figure 9 and Caravaggio's and Rembrandt's paintings are to the left in Figure 10. Moreover, cold colors are on the right in Figure 9 and Vermeer's paintings are to the right in Figure 10. Therefore, Caravaggio's and Rembrandt's paintings often feature warm colors such as red and brown. Moreover, Vermeer's paintings often feature cold colors such as blue and green.

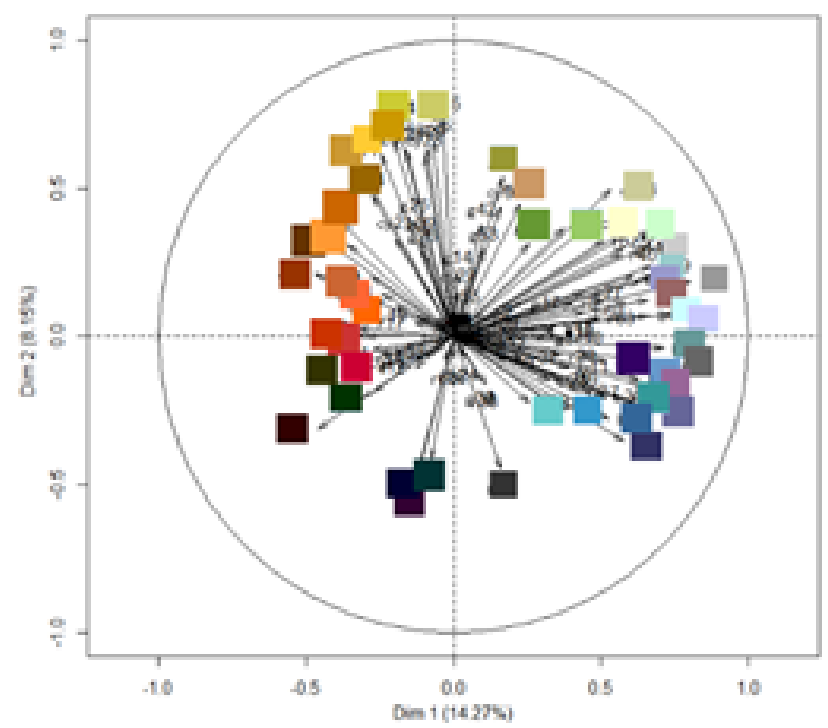

Fig. 9: Principal comp. loading (unigram, 126 works)

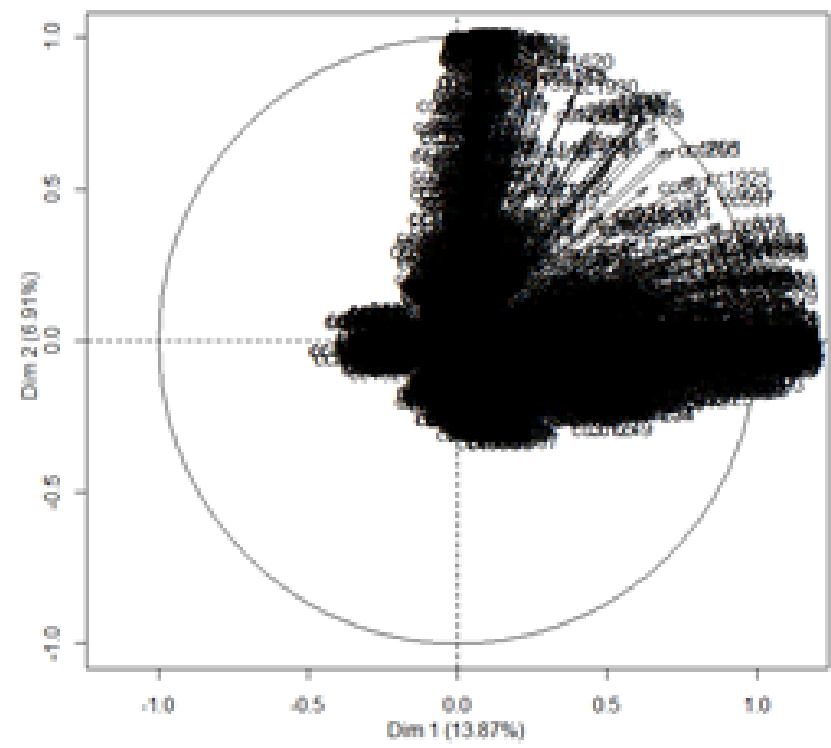

Fig. 11: Principal comp. loading (bigram, 130 works)

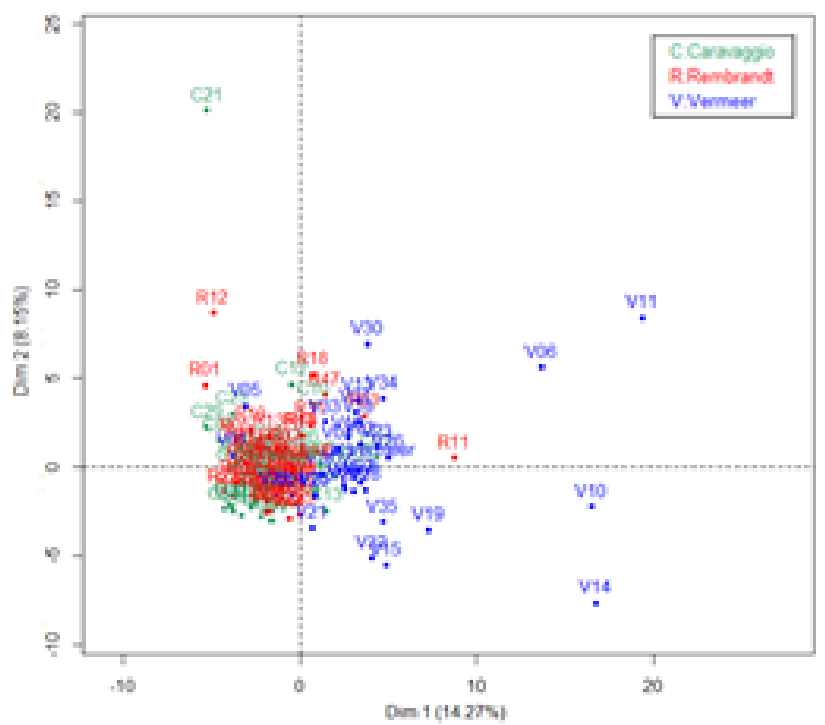

Fig. 10: Principal comp. of pc1 and pc2 (unigram, 126 works)

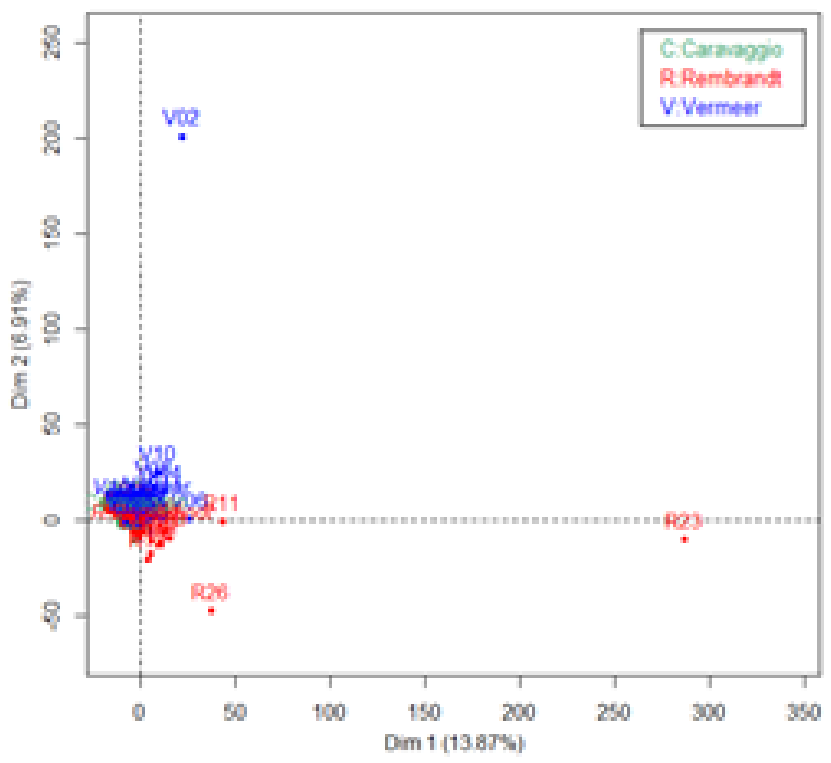

Fig. 12: Principal comp. of pc1 and pc2 (bigram, 130 works) 


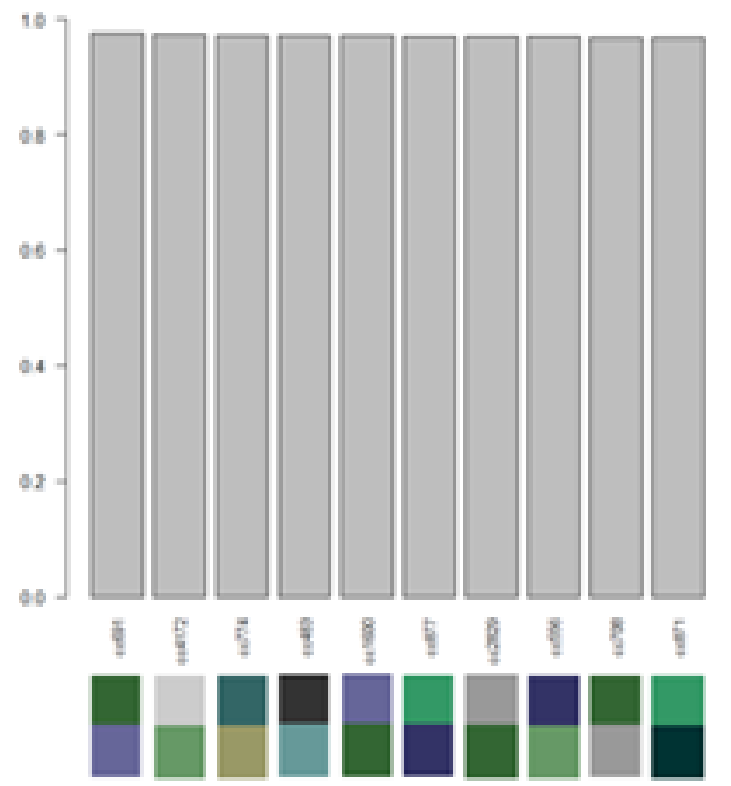

Fig. 13: Princ. comp. load. of $\mathrm{PC}_{1}$ (bigram, 130 works, top 10)

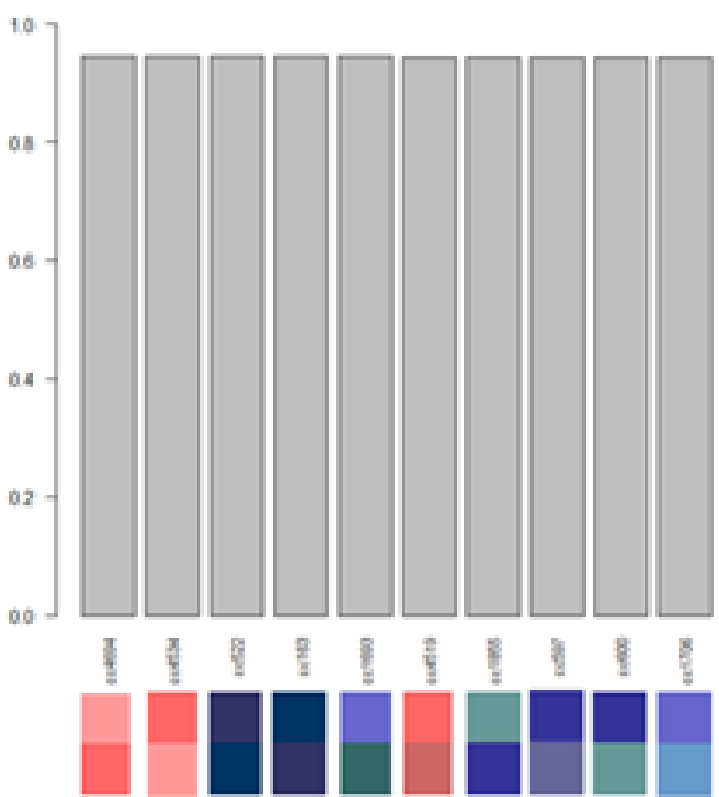

Fig. 14: Princ. comp. load. of PC2 (bigram, 130 works, top 10)

Figures 11, 12, 13, and 14 show PCA using 130 paintings and bigram data. Figure 11 shows a plot of principal component loading and Figure 12 that of the principal component. Figure 13 shows a bar graph of the first principal component and Figure 14 a bar graph of the second. Two paintings are out of position in Figure 11 because they have color-specific characteristics. The light blue and light green bigram are higher in rank in terms of the first principal component (Fig. 11 and 13). Rembrandt's painting no. 23 is at the top in terms of the first principal component (Fig. 12). Therefore, painting no. 23 features light blue and light green. On the contrary, red and dark blue bigrams are higher in rank in terms of the second principal component (Fig. 11 and 14). Vermeer's painting no. 2 is on top in terms of the second principal component (Fig. 12) and therefore features red and dark blue.

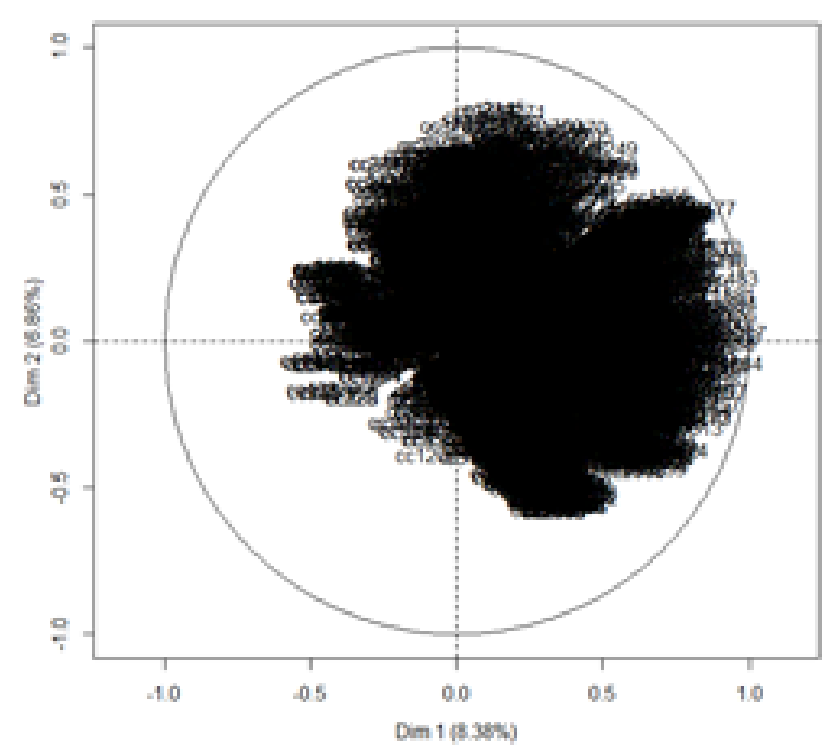

Fig. 15: Principal comp. loading (bigram, 128 works)

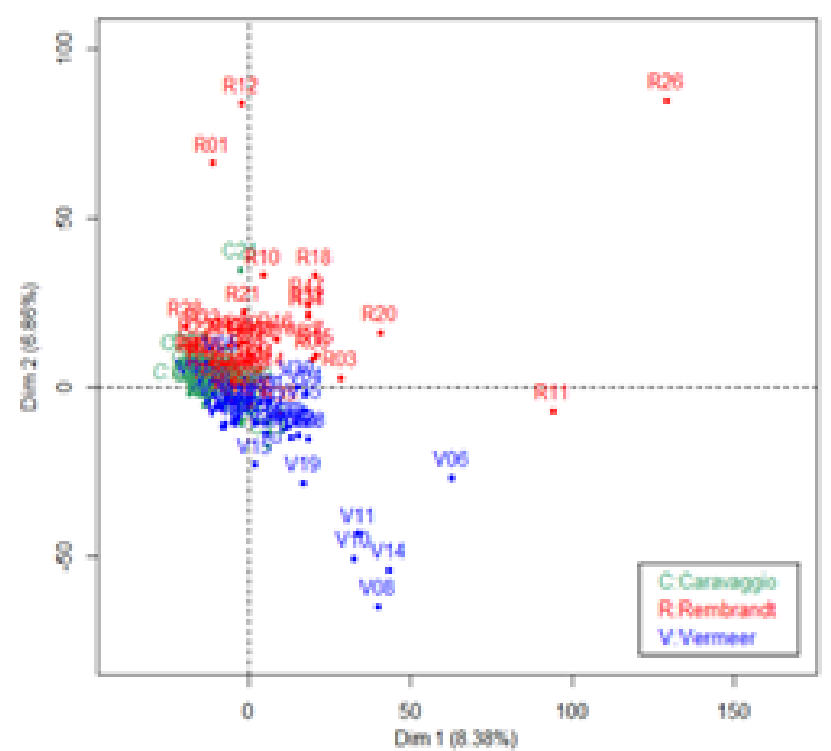

Fig. 16: Principal comp. of pc1 and pc2 (bigram, 128 works) 


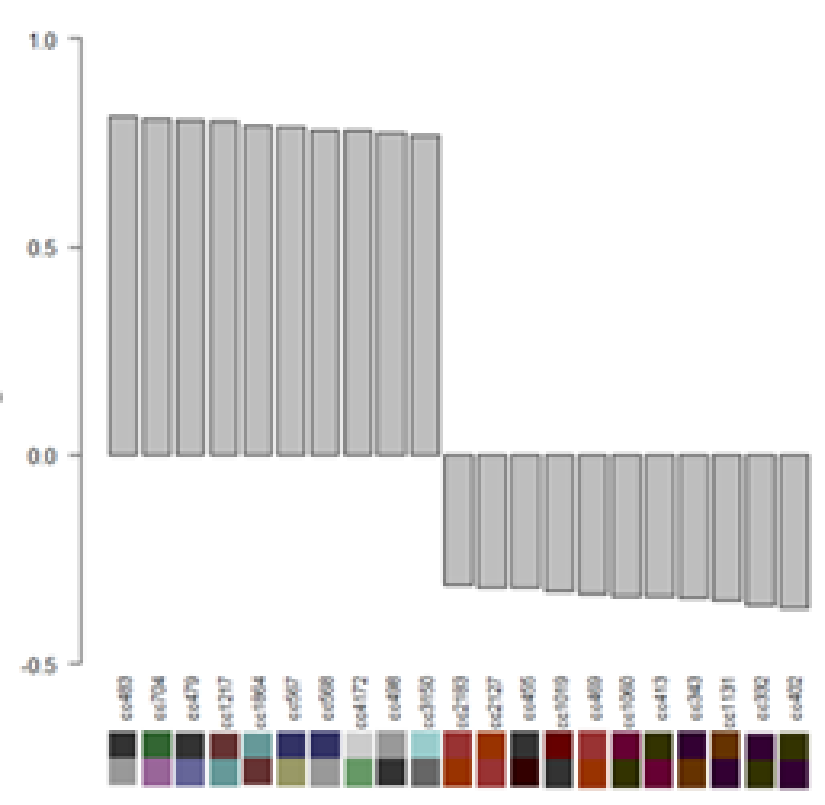

Fig. 17: Principal component loading of $\mathrm{PC}_{1}$ (bigram, 128 works, top and bottom 10)

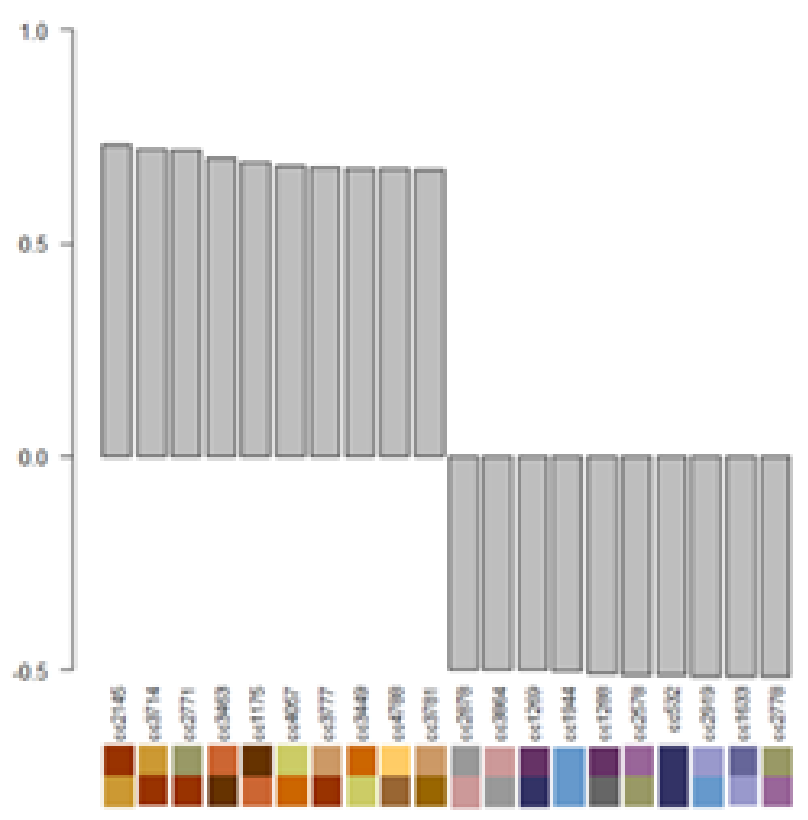

Fig. 18: Principal component loading of $\mathrm{PC} 2$ (bigram, 128 works, top and bottom 10)

Figures $15,16,17$, and 18 show the results of the PCA of 128 paintings on bigram data. Rembrandt's painting no. 23 and painting no. 2 by Vermeer were excluded. Figure 15 shows a plot of principal component loading and Figure 16 shows a plot of the principal component. Figure 17 shows a bar graph of the first principal component and Figure 18 that of the second principal component. In Figure 16 the paintings are divided into two groups based on the second principal component: one consisting of paintings by Caravaggio and Rembrandt and the other consisting of those by Vermeer. Warm colors in the bigram are higher in rank and cold colors lower in rank in terms of the second principal component (Fig. 17). Therefore, Caravaggio and Rembrandt's paintings often use warm colors while Vermeer's paintings often use cold colors. Moreover, bright colors in the bigram are higher in rank and dark colors are lower in terms of the first principal component (Fig. 16). Caravaggio's paintings are to the left in Figure 17 and Rembrandt's paintings are to the right. Therefore, Caravaggio used darker warm colors more often than Rembrandt.

\section{Conclusion}

In this research, we investigated the chromatic characteristics and styles of paintings by three famous artists of the Baroque era by using color information. The results indicate that Caravaggio's and Rembrandt's paintings often feature darker and warmer colors than Vermeer's paintings. Moreover, Vermeer often used bright and cold colors. Vermeer's painting no. 2 and painting no. 23 by Rembrandt have different color features. Vermeer's painting no. 2 features dark blue and dark red and painting no. 23 by Rembrandt features light blue and light green. This study also tested the effectiveness of color code for the analysis of paintings. The analysis using RGB captured differences in degrees of brightness of the paintings. However, the analysis using color code yielded differences in the features about paintings related to color. The color code was found to capture the characteristics of paintings and their styles better than RGB. The color code is thus effective for the feature analysis of paintings, and provides a new avenue in research on visual art. In future work, we plan to consider the motifs depicted in paintings using n-gram analysis. 


\section{Literature}

Martin BAILEY, Vermeer, Tokyo 2001.

Toshiyuki KASHIWAGI / Shunichiro OE, A Detection Method of Non-uniform Color Area Using Color Histogram, in: IEEJ Transactions on Electronics, Information and Systems 124/3 (2004), 666-671.

Michael KITSON, Rembrandt, Tokyo 2009.

Mingzhe JIN, Qualitative Data Analysis, Tokyo 2016. (金明哲『定性的データ分析』森北出版株式会社).

Naoki TAKAHASHI / Takashi SAKAMOTO / Toshikazu KATO, Color analysis of Image Photography of Interior Brand based on Extraction of Representative Colors and Clustered Color-features, in: Transactions of Japan Society of Kansei Engineering 15/1 (2016), 203-212.

Timothy WILson-SmItH, Caravaggio, Tokyo 2003. 


\title{
Travelogues: Fremdwahrnehmungen in Reisebe- richten 1500-1876
}

\author{
Eine computergestützte Analyse ${ }^{1}$
}

Doris Gruber [1], Martin Krickl [2], Lijun Lyu [3], Jan Rörden [4] \& Arno Strohmeyer [5]

[1] Institut für Neuzeit- und Zeitgeschichtsforschung (ÖAW)

doris.gruber@oeaw.ac.at, https://orcid.org/0000-0002-0512-100X

https://www.oeaw.ac.at/inz/personen/gruber-doris/

[2] Österreichische Nationalbibliothek

martin.krickl@onb.ac.at

https://www.onb.ac.at/

[3] Institut L3S, Leibniz Universität Hannover

lyu@|3s.de

https://www.I3s.de/ /yu/

[4] AIT Austrian Institute of Technology GmbH

jan.roerden@ait.ac.at, https://orcid.org/0000-0002-5824-8397

https://www.ait.ac.at

[5] Institut für Neuzeit- und Zeitgeschichtsforschung (ÖAW), Universität Salzburg

arno.strohmeyer@oeaw.ac.at, https://orcid.org/0000-0002-4077-8840

https://www.oeaw.ac.at/inz/personen/strohmeyer-arno/

Keywords: Travelogues, OCR-Post-Correction, Genre Classification, Metadata Enrichment, Early Modern Prints

\begin{abstract}
This international and interdisciplinary project creates algorithms for the semi-automatic search for and evaluation of printed travelogues in German, published in the time 1500-1876 and preserved in the holdings of the Austrian National Library.
\end{abstract}

\section{Einleitung}

Der Beitrag liefert erste Einblicke in ein im April 2018 gestartetes Projekt, das sich mit gedruckten Reiseberichten der Zeit 1500-1876 beschäftigt. Der Quellentypus bietet sich aufgrund des Inhalts, der großen Zahl an überlieferten Werken und deren Materialität an, mit Hilfe neuer Methoden der Digital

$1 \quad$ Das hier vorgestellte Projekt findet dank der Finanzierung durch den FWF (I 3795-G28) und die DFG (398697847) statt. Beteiligt sind: das Institut für Neuzeit- und Zeitgeschichtsforschung an der Österreichischen Akademie der Wissenschaften, das Austrian Institute of Technology, die Österreichische Nationalbibliothek und das Forschungszentrum L3S an der Universität Hannover. Weitere Details zum Projekt und künftige Publikationen werden über die Projekthomepage angekündigt: http://www.travelogues-project.info/ (21.03.2019). 
Humanities analysiert zu werden. Inhaltlich sind Reiseberichte besonders relevant, weil sie Wahrnehmungen von „Fremdheit”, wie geographische Regionen, Kulturen und Religionen, transportieren. Diese Fremdwahrnehmungen wurden stark von den an ihrer Produktion beteiligten Personen geprägt, was der Forschung zahlreiche Rückschlüsse erlaubt: Es kann etwa darauf geschlossen werden, wie diese Personen, respektive bestimmte Kulturkreise, mit „Fremdheit” umgingen und welche Faktoren dafür verantwortlich waren, dass Stereotype und Vorurteile geprägt wurden und sich in diachroner Perspektive wandelten. Materiell gesehen stehen die gedruckten Texte der Reiseberichte im Fokus, deren inhaltliche Ausprägungen und materielle Formen (Typen- und Textsatz) zwar äußerst heterogen sind, aber deutlich besser und leichter in maschinenlesbare Form übersetzt werden können als andere Medienformen wie die ebenfalls in den gedruckten Reiseberichten erhaltenen Bilder oder auch Handschriften.

Reiseberichte wurden von der Forschung zwar schon vielfach untersucht, bisherige Studien beschränkten sich aber meist auf ein relativ kleines Korpus von selten mehr als einem Dutzend Reiseberichten. ${ }^{2}$ Obwohl sich heuristische Ansätze als fruchtbar erwiesen, ${ }^{3}$ fehlt es an quantitativen Analysen. Diese können einerseits bisherige Forschungsthesen überprüfen und andererseits dominante Aspekte (wie führende Verlage, Produktionsspitzen, regionale und thematische Schwerpunkte) aufzeigen, die durch rein qualitative Zugänge nicht ersichtlich sind. ${ }^{4}$

Deshalb wird im Zuge dieses Forschungsprojekts der Frage nachgegangen, wie neue digitale Analyseverfahren genutzt werden können, um gedruckte Reiseberichte und insbesondere darin erhaltene Fremdwahrnehmungen quantitativ und qualitativ zu untersuchen. Das Projekt setzt hierfür auf mehreren Ebenen an, die mitunter parallel bearbeitet werden. Bislang beschäftigten wir uns vorwiegend mit der Korpusbildung, der Aufbereitung der Metadaten zu den Drucken und der Aufbesserung der mit Optical Character Recognition (OCR) ausgelesenen Texte.

\section{Korpusbildung und Metadatenaufwertung}

Die Korpusbildung ist bereits weit vorangeschritten und erfolgt durch etablierte Methoden der Geisteswissenschaften, die durch neue digitale Erhebungsverfahren ergänzt werden. Das Korpus beschränkt sich grundsätzlich auf deutschsprachige Reiseberichte im Bestand der Österreichischen Nationalbibliothek (ÖNB), die zwischen 1500 und 1876 gedruckt wurden. Obwohl durch die Begrenzung auf die Bestände einer Bibliothek nicht die Gesamtheit der Reiseliteratur untersucht werden kann, eignen sich jene der ÖNB ausgesprochen gut für exemplarische Analysen mit Methoden der Digital Humanities, da die Bestände im Rahmen des Projektes Austrian Books Online (ABO) zwischen 2011 und 2018 mehrheitlich digitalisiert (ca. 600.000 Objekte) und die Texte mittels OCR-Software ausgelesen wurden (verfügbar via $\mathrm{SACHA}^{5}$ ).

2 Beispielhaft für eine weitreichende, wenn auch unvollständige Bibliographie: Carlo SALZANI / Steven TÖTÖsY DE ZEPETNEK, Bibliography for Work in Travel Studies, in: Library Series, CLCWeb: Comparative Literature and Culture (Version 14.09.2016), online unter: https://docs.lib.purdue.edu/clcweblibrary/travelstudiesbibliography (21.03.2019).

3 Beispielhaft: Bekim Agal / Stephan ConermanN, Hg., „Wenn einer eine Reise tut, hat er was zu erzählen“: Präfiguration Konfiguration - Refiguration in muslimischen Reiseberichten, Berlin 2013; Almut HöFERT, Den Feind beschreiben. „Türkengefahr" und europäisches Wissen über das Osmanische Reich 1450-1600, Frankfurt am Main / New York 2003; Michiel VAN Groesen, The Representations of the Overseas World in the De Bry Collection of Voyages (1590-1634), Boston / Leiden 2008.

4 Franco MORETTI, Distant reading, London / New York 2013.

5 ÖNB, Hg., Simple Access to Cultural Heritage Assets (SACHA), Online-Datenbank, online unter: https://iiif.onb.ac.at/ (21.03.2019). 
Die Korpusbildung im klassischen Sinne fand im Katalogsystem über kombinierte Suchanfragen zu Titelinformationen und Schlagwörtern ${ }^{6}$ statt, deren Ergebnisse fachlich kuratiert und durch Erhebungen in Bibliographien zum Thema laufend ergänzt werden. ${ }^{7}$ Das Korpus selbst wird direkt im Bibliothekssystem ALMA erstellt, wodurch die Daten zum einen systemintern effizient weiter bearbeitet werden können und zum anderen - im Gegensatz zu Stand-Alone-Datenbanken - nachhaltig gesichert werden. Außerdem stehen dadurch sowohl im User-Interface (Primo) als auch über die LOD (Linked Open Data)-Schnittstellen stets aktuelle Datensätze zur Verfügung.

Das Ergebnis dieser Erhebungen ist ein Basiskorpus von circa 3.200 Bänden an Reiseberichten (16. Jh.: 77, 17. Jh.: 180, 18. Jh.: 780, 19. Jh.: 2.200). Allerdings sind die Titel- und Schlagwortsuchen nur begrenzt aussagekräftig, da sich die Qualität der historisch gewachsenen Metadaten (wie Angaben zu Titeln, Autorlnnen oder Druckorten) im Bibliothekssystem als äußerst heterogen erwies und Fehler ans Tageslicht traten. Bei der Migration vom handschriftlichen Zettelkatalog zum digitalen Bibliothekssystem durchliefen diese diverse Bearbeitungsprozesse, die zu unzähligen Dubletten, verkürzten Titeln, mangelnden oder oberflächlichen Beschlagwortungen und fehlenden Genre-Klassifizierungen führten. Die in ihrem Aufwand nicht zu unterschätzende Datenbereinigung (Metadata-Cleaning) und die darauf aufbauende systematische Datenaufwertung (Metadata-Enrichment) kann im Zuge des TraveloguesProjekts zwar nicht für den Gesamtbestand der ÖNB erfolgen, wohl aber für das erhobene Korpus an Reiseberichten. Bis Ende 2019 werden qualitativ hochwertige, kritisch geprüfte Metadaten im UserInterface des Bibliothekskataloges ${ }^{8}$ und über LOD-Schnittstellen in diversen Formaten (RDF, Bibframe, RDA) zu unserem Korpus abrufbar sein. Für weitere Analysen, Visualisierungen und Annotationen der Metadaten stehen die ÖNB-LibraryLabs zur Verfügung. ${ }^{9}$

\section{Classifier}

Aufgrund der generellen Limitierungen durch Titel- und Schlagwortsuchen, die durch die heterogene Qualität der Metadaten im Bibliothekssystem der ÖNB weiter verstärkt werden, und weil bisher keine Gesamtbibliographie zu deutschsprachigen Reiseberichten der Zeit 1500-1876 vorliegt, können durch die oben beschriebenen Vorgehensweisen unmöglich alle relevanten Werke im Bestand der ÖNB identifiziert werden. Deswegen wird im Rahmen dieses Projektes ein weitgehend automatisches Verfahren zur Auffindung bislang unbekannter Reiseberichte entwickelt, das sich an inhaltlichen Ähnlichkeiten der OCR-ausgelesenen Texte orientiert und von uns als „Classifier” bezeichnet wird.

Zur Implementierung von Methoden des Machine Learning wurde zunächst eine Groundtruth erstellt, die sich aus dem wie oben beschrieben intellektuell identifizierten Korpus von ca. 3.200 Reiseberichten sowie derselben Zahl an Negativbeispielen (d.h. keine Reiseberichte) zusammensetzt. Letztere wurden durch ein randomisiertes Verfahren erhoben und ebenfalls intellektuell überprüft. Anhand der Unterschiede zwischen diesen beiden Klassen („Reisebericht“ und „Andere“) wurde eine SVM (Support Vector Machine) an einem Trainingsset trainiert. Um sprachlichen und inhaltlichen Wandlungsprozessen im Laufe des Untersuchungszeitraums zumindest teilweise gerecht zu werden, wurde das Korpus in vier Untersets zu je einem Jahrhundert gegliedert, zu denen jeweils eigene Modelle trainiert wurden. Mit

6 Gesucht wurde nach unterschiedlichen, trunkierten Schreibweisen der Begriffe „Reise“ und „Fahrt“, sowie nach den Schlagworten „Reisebericht" und „Forschungsreise“.

7 Beispielhaft: Eutiner Landesbibliothek, Hg., Bibliografie und Katalog Reiseliteratur, online unter: https://lb-eutin.kreis-oh. de/index.php?id=275 (21.03.2019).

8 ÖNB, Hg., Bibliothekskatalog (Primo), online unter: https://search.onb.ac.at/ (21.03.2019).

9 ÖNB, Hg., Labs, online unter: https://labs.onb.ac.at/de/ (21.03.2019). 
dieser Methode konnten die Werke aus unserem Testset mit einer Genauigkeit zwischen 88 und 94 Prozent korrekt zugeordnet werden.

Um weitere Werke zu identifizieren, die unserer Definition von Reisebericht entsprechen, klassifizierten wir mit Hilfe dieser Modelle zudem alle bisher von uns nicht evaluierten, OCR-ausgelesenen Texte im Bestand der ÖNB. Für jedes Werk erhielten wir neben der binär definierten Klasse auch die Konfidenzwerte des Modells, sodass ein Ranking entstand. Um die Effektivität unserer Methode zu evaluieren, wurden für jedes Jahrhundert die jeweils ersten 100 als vermeintliche Reiseberichte klassifizierten Werke intellektuell überprüft. Zwischen 10 und 21 entsprachen jeweils unserer Definition („true positives") und insgesamt konnten $56^{10}$ uns zuvor unbekannte Reiseberichte aufgefunden werden. Die vergleichsweise geringe Zahl an „true positives“ spricht einerseits für die Effektivität der klassischen geisteswissenschaftlichen Erhebungsmethoden, andererseits gründet sie in der großen Zahl an Texten, die als Reiseberichte klassifiziert wurden, innen inhaltlich auch stark ähneln, aber eben keine Reiseberichte sind (wie historiographische und geographische Werke oder Reiseführer). Deshalb arbeiten wir bereits an einer feineren Klassifikation, um beispielsweise mittels Named Entity Recognition und Topic Modeling unsere Modelle zu verbessern.

\section{OCR-Nachbearbeitung}

Neben der Korpusbildung und Metadatenbereinigung werden im Projekt derzeit die maschinenlesbaren Texte aufgebessert. Wenngleich sich die OCR-Software in den letzten Jahren zunehmend verbesserte, sind die damit erzeugten Transkriptionen weiterhin stark fehlerhaft, wenn auch in unterschiedlicher Ausprägung. Die Fehlerquote ist etwa abhängig von der Textstruktur im Digitalisat (z.B. Ein- und Mehrspaltigkeit) oder dem Vorhandensein grafischer Elemente. Weitere Herausforderungen sind der Einfluss des diachronen Sprachwandels und der Mangel an stilistischer und orthographischer Standardisierung während der Untersuchungsperiode (z.B. die Varianzen $u / v, u / j$ und $s / I$ ).

Die Nachbearbeitung der OCR-ausgelesenen Texte in der ÖNB erfolgt im Travelogues-Projekt mit Hilfe Neuronaler Netzwerke (NN). Die Groundtruth hierfür stammt aus einem Testset von 13 Büchern, deren Volltexte sowohl in der OCR-ausgelesenen Form in der ÖNB vorliegen als auch im Zuge des Projekts „Deutsches Textarchiv” (DTA) ${ }^{11}$ manuell transkribiert wurden. Aus diesen Texten wurden $\mathrm{N}$-Gram-Wortsequenzen extrahiert, mit Hilfe von Locality Sensitive Hashing (LSH) fehlerhafte Stellen in den OCR-Texten identifiziert und mit den sauberen Transkriptionen gepaart (insgesamt ca. 800.000 Paare). An dieser Groundtruth wurden verschiedene Encoder-Decoder-Modelle gemeinsam trainiert (basierend auf einem gewöhnlichen Encoder-Decoder-Modell wurde vor allem mit Sequence Representation und Weighted Attention experimentiert) und auf Character-, N-Gram-Character und BPESequence-Level getestet. Die besten Ergebnisse wurden auf dem Character-Level erzielt. Weitere Experimente mit Global Attention und Hierarchical Attention führten dazu, dass unser Character Global Attention Modell die Word Error Rate (WER) der OCR-gelesenen Texte im Schnitt um 30 Prozent reduzieren kann und ein Multiple Attention Modell die Ergebnisse sogar weiter verbessert. Dennoch kamen wir zum Schluss, dass kein einzelnes Modell alle Probleme lösen kann, weswegen weitere Experimente stattfinden müssen, bevor die Neuronalen Netzwerke das gesamte Textkorpus des

10 Diese Zahl betrifft die bisherigen Evaluierungen vom 16. bis zum 18. Jahrhundert und umfasst auch Reiseberichte, die bei der Evaluierung der Negativbeispiele zufällig gefunden wurden; Ohne diese sind es 47.

11 Berlin-Brandenburgischen Akademie der Wissenschaften, Hg., Deutsches Textarchiv. Grundlage für ein Referenzkorpus der neuhochdeutschen Sprache, Berlin 2019, online unter: http://www.deutschestextarchiv.de/ (21.03.2019). 
Travelogues-Projekts aufbessern werden. Wir erwarten, dass durch diese Vereinheitlichung des Textmaterials die oben beschriebene Klassifikation, wie auch alle künftigen Analysen, bessere Ergebnisse erzielen.

\section{Resümee und Ausblick}

Durch den Einbezug von Machine Learning Verfahren wurden bereits vielversprechende Zwischenergebnisse bei der Korpusbildung und der OCR-Nachbearbeitung erzielt: Wir konnten Reiseberichte identifizieren, die durch klassische Erhebungsmethoden der Geisteswissenschaften nicht auffindbar waren und die Qualität der OCR-gelesenen Texte wurde an einem Testset deutlich verbessert. Die Arbeiten sind jedoch in beiden Bereichen noch nicht abgeschlossen und bedürfen weiterer Analysen.

Darüber hinaus werden wir uns künftig der semi-automatisierten Analyse der textuellen Inhalte der Reiseberichte zuwenden. Im Zentrum werden hierbei intertextuelle Relationen zwischen den einzelnen Reiseberichten (v.a. in-/direkte Zitate, Übernahmen von Textstellen und Allusionen) sowie die in den Reiseberichten tradierten Fremdwahrnehmungen stehen.

\section{Literaturverzeichnis}

Bekim Agal / Stephan Conermann, Hg., „Wenn einer eine Reise tut, hat er was zu erzählen“: Präfiguration Konfiguration - Refiguration in muslimischen Reiseberichten, Berlin 2013.

Berlin-Brandenburgischen Akademie der Wissenschaften, Hg., Deutsches Textarchiv. Grundlage für ein Referenzkorpus der neuhochdeutschen Sprache, Berlin 2019, online unter: http://www.deutschestextarchiv.de/ (21.03.2019).

Eutiner Landesbibliothek, Hg., Bibliografie und Katalog Reiseliteratur, online unter: https://lb-eutin.kreis-oh.de/ index.php?id=275 (21.03.2019).

Almut HÖFERT, Den Feind beschreiben. „Türkengefahr“ und europäisches Wissen über das Osmanische Reich 1450-1600, Frankfurt am Main / New York 2003.

Franco MORETTI, Distant reading, London / New York 2013.

ÖNB, Hg., Bibliothekskatalog (Primo), online unter: https://search.onb.ac.at/ (21.03.2019).

ÖNB, Hg., Labs, online unter: https://labs.onb.ac.at/de/ (21.03.2019).

ÖNB, Hg., Simple Access to Cultural Heritage Assets (SACHA), Online-Datenbank, online unter: https://iiif.onb. ac.at/ (21.03.2019).

Carlo SALZANi / Steven TöTÖsY DE ZEPETNEK, Bibliography for Work in Travel Studies, in: Library Series, CLCWeb: Comparative Literature and Culture (Version 14.09.2016), online unter: https://docs.lib.purdue.edu/clcweblibrary/travelstudiesbibliography (21.03.2019).

Michiel VAN GRoesen, The Representations of the Overseas World in the De Bry Collection of Voyages (15901634), Boston / Leiden 2008. 


\title{
Bibliotheksdaten in DH-Forschungsprojekten
}

\author{
EINDRÜCKE des ROUNd TABLES BeI DER DHA2018
}

\section{Markus Bürscher [1] \& Lina Maria Zangerl [2]}

[1] Diözesan- und Universitätsbibliothek (DUB) der Katholischen Privat-Universität Linz m.buerscher@ku-linz.at https://ku-linz.at/bibliothek/, https://orcid.org/0000-0003-0398-9144

[2] Universität Salzburg - Literaturarchiv Salzburg linamaria.zangerl@sbg.ac.at https://uni-salzburg.at/literaturarchiv, https://orcid.org/0000-0001-9709-3669

Keywords: Library Data, Authority Files, Embedded Librarianship, Digital Preservation, Collaboration

\begin{abstract}
The aim of the roundtable on the subject of library data in DH research projects, held as part of dha2018, was to explore the intersection between DH and libraries and to identify some problems as well as opportunities.
\end{abstract}

Die 5. Jahreskonferenz der Digitalen Geisteswissenschaften in Österreich stand unter dem Motto „Empowering researchers" und widmete sich u. a. der Frage, wie das Netzwerk zwischen Disziplinen, Forschungsgegenständen und Forschenden in den $\mathrm{DH}$ bestmöglich zu stärken ist. Zu diesem Netzwerk gehören ganz wesentlich auch die Bibliotheken und ihre vielfältigen Ressourcen. Die Schnittstellen zwischen DH und Bibliotheken auszuloten und einige Probleme, aber auch Chancen festzumachen, war Ziel des Round Tables zum Thema Bibliotheksdaten in DH-Forschungsprojekten, der im Rahmen der dha2018 am 29. November im UNIPARK Nonntal stattfand. Es diskutierten Markus Bürscher von der Diözesan- und Universitätsbibliothek Linz, der Geschäftsführer der Österreichischen Bibliothekenverbund und Service $\mathrm{GmbH}$, Wolfgang Hamedinger, Peter Hinkelmanns, wissenschaftlicher Mitarbeiter der Mittelhochdeutschen Begriffsdatenbank an der Universität Salzburg und Martin Krickl, Datalibrarian an der Österreichischen Nationalbibliothek. Lina Maria Zangerl vom Literaturarchiv Salzburg moderierte die Veranstaltung.

Im Gespräch kamen verschiedene Grundfragen der Zusammenarbeit zwischen Bibliotheken und $\mathrm{DH}$-Forschungsprojekten zur Sprache. Die Diskutanten nannten zunächst positive Beispiele für gelingende Kooperationen, wie etwa die derzeit entstehende digitale Jägerstätter-Edition ${ }^{1}$ oder das FWF-Projekt „Travelogues“2 an der Österreichischen Nationalbibliothek. Die kurzen Eindrücke aus der Berufspraxis verdeutlichten zum einen, welche thematische, technische und organisatorische Spannweite solche Projekte zwischen Forschung und Bibliothek umfassen, und zum anderen, auf welche Herausforderungen die Beteiligten dabei treffen.

1 https://ku-linz.at/forschung/franz_und_franziska_jaegerstaetter_institut/ (10.05.2019).

2 http://www.travelogues-project.info/, siehe hierzu auch den Beitrag im vorliegenden Band. 
Das erste diskutierte Problemfeld betraf die Nutzung von Daten, die in Bibliotheken bereits vorhanden sind. So sind bibliographische Metadaten etwa in vielen korpusbasierten DH-Forschungen ein essentieller Bestandteil, dennoch ist weder die Nachnutzung von Bibliotheksdaten durch die Projekte noch umgekehrt die Erweiterung dieses Bibliotheksdatenbestands durch Forschungsergebnisse aus den Projekten derzeit Standard. Im Mittelpunkt der Diskussion stand die Zugänglichkeit und Bearbeitbarkeit der von Bibliotheken getragenen Datenbanken, insbesondere der Gemeinsamen Normdatei GND: In welcher Weise können DH-Projekte nicht nur die GND für ihre Forschungen nutzen, sondern diese auch mit eigenen Eingaben erweitern und ergänzen - woraus die Bibliotheken wiederum Gewinn ziehen können? Peter Hinkelmanns wies in diesem Zusammenhang zurecht auf die mangelnden Kollaborationsmöglichkeiten für Forschende hin, die im Moment selbst nicht redaktionell in die GND eingreifen können, aber auch keine veralteten Datensätze nutzen möchten, die ihre eigenen Forschungserkenntnisse nicht wiedergeben. Die Aussage von Wolfgang Hamedinger, hier im Österreichischen Bibliothekenverbund künftig zu größerer Offenheit beitragen zu wollen, sorgte für Zustimmung und Freude unter den Teilnehmern der Diskussion, wie auch im Publikum.

Auch neue Entwicklungen im Bereich der Datenformate in Bibliotheken (z.B. BIBFRAME) und die Rolle des Semantic Web wurden in der Runde diskutiert. Es herrschte Konsens darüber, dass sich Datenmodelle sowohl in DH-Projekten als auch in Bibliotheken verstärkt in Richtung Semantic Web entwickeln und an dieser Schnittstelle großes Potential bieten. Es gibt bereits mehrere Arbeitsgruppen, etwa an der Österreichischen Nationalbibliothek oder im Rahmen des nationalen Kompetenznetzwerks für digitale Edition (KONDE) ${ }^{3}$, die sich in diesem Kontext insbesondere mit Linked Open Data (LOD) auseinandersetzen und deren Ergebnisse den Austausch zwischen Bibliotheksdaten und Daten in Forschungsprojekten befördern können.

Weitere besprochene Herausforderungen ergeben sich aus strukturellen und institutionellen Umständen. In der Diskussion wurde deutlich, dass Kommunikation und Kooperation zwischen Forschungsprojekten und Bibliotheken dort besonders gut funktioniert, wo eine große organisatorische Nähe zur Bibliothek als (Träger-)Institutionen besteht, beispielsweise in Fach- und Institutsbibliotheken. Im universitären Wissenschaftsbetrieb ist in Österreich aber eine strikte Trennung zu beobachten zwischen wissenschaftlichem Personal, aus dem sich Projektmitarbeiterlnnen rekrutieren, und allgemeinem Personal, dem die BibliothekarInnen angehören. Diese personalpolitische Gegebenheit erschwert in der Praxis oft eine übergreifende Zusammenarbeit in Projekten. So ist etwa das in der Diskussion wiederholt präsente und in vielen Fällen sehr wünschenswerte Konzept des „embedded librarian“4 eine Strategie zur direkten Einbettung von BibliothekarInnen in Forschungsgruppen - in einer solchen Struktur nur schwer umzusetzen. Besser ermöglichen dies große Forschungsbibliotheken, die selbst drittmittelgeförderte Projekte durchführen und in diesem Rahmen auch „embedded librarians“ beschäftigen. Martin Krickl konnte als Datalibrarian in einem DH-Forschungsprojekt an der Österreichischen Nationalbibliothek beim Round Table Einblick in eine solche Funktion geben.

Der institutionelle Problemhorizont kennt aber noch eine weitere Ebene, die von Johannes Stigler aus dem Publikum ins Treffen geführt wurde: Bibliotheken sind gefordert, im Umgang mit DH-Projekten verstärkt als Garanten von Nachhaltigkeit aufzutreten. Dies insbesondere deshalb, weil Bibliotheken

3 http://digitale-edition.at (10.05.2019).

4 Kathy DRESES / Nadine HofMANN, Academic Embedded Librarianship: An Introduction, in: Public Service Quarterly 6 (2010), 75-82, DOI: 10.1080/15228959.2010.498773. 
die größte Erfahrung und das größte Interesse daran haben, Forschungsdaten ebenso wie die Präsentationsformen von DH-Projekten langfristig zu sichern und zur Verfügung zu stellen. Verwaltung, Betreuung und Entwicklung von DH-Plattformen an Bibliotheken anzusiedeln, wäre daher sinnvoll und sollte stärker forciert werden. Die Frage nach Strategien für Forschungsdaten aus DH-Projekten, die in Bibliotheken und ihren Repositorien aufbewahrt werden sollten, musste im Detail offen bleiben. Es wurde deutlich, dass hier im Vorfeld auch Erwägungen zu Urheber- und Datenschutzrechten eine Rolle spielen müssen.

Aus dem Publikum kam abschließend von Katharina Zeppezauer-Wachauer auch der Einwurf, dass bzw. ob man analog zum „embedded librarian“ in DH-Projekten auch über einen „embedded digital humanist“ in Bibliotheken nachdenken sollte. Dieser Gedanke greift einen zentralen Aspekt auf, der in der Diskussion deutlich wurde: Eine stärkere Zusammenarbeit von DH-ProjektmitarbeiterInnen und BibliothekarInnen bietet im gegenwärtigen österreichischen Wissenschafts- und Bibliotheksbetrieb großes und meist ungehobenes Potential. Aufgrund fehlender struktureller Verankerungen und mangelnder Förderungen ist dieses Potential aber oft auf das persönliche Engagement der Beteiligten angewiesen, die eigenständig für einen regen Austausch zwischen Bibliothek und Wissenschaft sorgen und wissen, dass beide voneinander profitieren können.

\section{Literaturverzeichnis}

Kathy Drewes / Nadine Hofmann, Academic Embedded Librarianship: An Introduction, in: Public Service Quarterly 6 (2010), 75-82, DOI: 10.1080/15228959.2010.498773.

http://digitale-edition.at (10.05.2019).

https://ku-linz.at/forschung/franz_und_franziska_jaegerstaetter_institut/ (10.05.2019).

http://www.travelogues-project.info/ (10.05.2019). 


\title{
The Role of Analog Publications in a Digital World
}

\author{
The Archaeological Case Study Celsus
}

Nicole M. High-Steskal

Austrian Archaeological Institute of the Austrian Academy of Sciences
nicole.high-steskal@oeai.at
https://www.oeaw.ac.at/oeai/das-institut/team/zentrale-wien/high-steskal-nicole/,
https://orcid.org/0000-0002-9823-5507

Keywords: Archaeology, Urban Gazetteer, Linked Open Data, Open Access, Ephesos

\begin{abstract}
CELSUS is an archaeological digitization project focused on the creation of a spatially driven digital edition of the monograph series Forschungen in Ephesos (FiE), a publication series edited by the Austrian Archaeological Institute (OeAl). It was first started in 1906 and represents the most important results of the Austrian excavations in Ephesos to date. However, many of the older volumes no longer are available and the series is difficult to use for individuals unfamiliar with the long research history of Ephesos. Yet, the information in these volumes is of considerable interest to scholars because they document all archaeological engagement with the monuments and people of the area, often provide detailed descriptions of the landscape, including of remains that no longer are visible, furthermore, they include full catalogs of all finds, and, therefore, constitute primary evidence for all studied contexts and finds in Ephesos.
\end{abstract}

The ancient city of Ephesos, an archaeological site located along the western coast of modern-day Turkey, has been the object of intense study by the Austrian Archaeological Institute since $1895 .{ }^{1}$ The past 125 years have led to the accumulation of extremely large quantities of information from a single archaeological site. The collected information documents human engagement with the geographic location from the $6^{\text {th }}$ millennium BCE to the modern period with a specific focus on the $7^{\text {th }}$ century BCE to the $14^{\text {th }}$ century CE. The bulk of the information collected up until the late 1990 s is analog while the first digital data were created in the 1990s and has increased over the years. ${ }^{2}$ The information has been documented in a variety of formats ranging from analog, digitized, to born digital. Analog information is available through the plentiful publications on Ephesos as well as the large in-house archive safeguarding the diaries of excavation directors and the documentation of the excavations encompassing

1 For an overview of the site, see: Sabine LADSTÄTTER, Hafen und Stadt von Ephesos in hellenistischer Zeit, in: Jahreshefte des Österreichischen Archäologischen Institutes 85 (2016), 233-272.

2 For the use of the terms data, dataset,... see: Adela SовоткоvA, Sociotechnical Obstacles to Archaeological Data Reuse, in: Advances in Archaeological Practice 6/2 (2018), 118; Ben MARWICK / Suzanne BIRCH, A Standard for the Scholarly Citation of Archaeological Data as an Incentive to Data Sharing, in: Advances in Archaeological Practice 6/2 (2018), 126f. 
maps, sketches, and photographs. A large part of the historic photographs has already been digitized and is available through an internal image database. A Geographic Information System was also implemented in order to better manage the spatial information documented during the excavation process.

Using this information poses several challenges. The first obvious issue is one of accessibility: the first Austrian essays on Ephesos were published in the 1890s in a limited number and only very few libraries still have a copy of these publications. Another challenge is the amount of information. The collection of data has increased exponentially over the years so that it is no longer possible for a single person to retain the information in its entirety. This has led to unfortunate situations where already documented information is "redocumented" or already studied aspects are again studied without any consideration of the previous results. The third considerable issue is that of language. Not only are the volumes largely written in complex German but over the years spelling has changed as has the evaluation of monuments. In particular, the first interpretation and naming of monuments has not always stood the test of time. Keeping track of these name changes can be a challenge to all, particularly to scholars not fully fluent in German. The consequence of these three issues is that it puts scholars at a disadvantage who do not have a solid command of German and those who do not have access to fully stocked libraries.

In order to move forward in the study of Ephesos it is necessary to manage the existing information in a more efficient way. CELSUS marks the first step of the OeAl towards making its vast collection of literature, archival material, and images openly available and we are partnering with digital humanities specialists at several institutions in Austria and Germany. In a pilot project we are focusing on the most important publication series on Ephesos, the Forschungen in Ephesos (FiE), which currently include 48 volumes. In collaboration with the Verlag der Österreichischen Akademie der Wissenschaften, we are working with Propylaeum ${ }^{3}$ of the Heidelberg University Library, a specialized information service for Classical studies and archaeology, which is scanning the entire FiE for us, supporting us in the application of automatic text recognition, and archiving the scans and associated metadata. Propylaeum follows all current best practices of Linked Open Data and implements various semantic frameworks (CIDOC-CRM) and metadata formats. Furthermore, through the use of persistent identifiers the digital editions can be sustainably referenced.

In keeping with the tradition of the $\mathrm{FiE}, \mathrm{CELSUS}$ is primarily focusing on a topographical approach since the geographic context of objects and the location of archaeological sites is often the first point of inquiry in archaeology. In the following I would like to illustrate several of the challenges we face in working with the geo-spatial information of Ephesos.

The FiE volumes contain a wide variety of spatial information. The most obvious sources are the maps contained in every volume of the $\mathrm{FiE}$. One of the first fairly precise maps of Ephesos was published in the first FiE in 1906 and was created by Hauptmann Anton Schindler. ${ }^{4}$ This map illustrates a first hurdle that we face in sorting our data: the map features several spelling variants and somewhat different terminology than that used in the text of the volume. The text and the map were created by two different people with differing objectives and academic backgrounds. Schindler created the map based on his training in geodesy and considerable interviews with locals while Benndorf's text approaches the landscape from a historic/archaeological viewpoint. In some instances the topographic terms are only

3 https://www.propylaeum.de/home/ (01.04.2019).

4 Otto BenNDORF, Hg., Forschungen in Ephesos, Wien 1906. 
mentioned on this one map. Again, the text by Otto Benndorf refers to sites not indicated on the map. A lack of standardization in the geo-spatial terminology up until the 1960s has resulted in a multitude of terms. In a first step it will be necessary to resolve the geo-spatial terminology of the site. This includes extracting topographic terms from the digitized FiE, particularly from the maps. The extracted terms will be fed into a geo-spatial database currently in development. The terms will be normalized to increase retrievability of information and reconciled with existing ontologies, such as Pleiades or the DAl gazetteer. This process will not only include the German concepts found in the main FiE-corpus but will also include English and Turkish variants. Through annotation of the geo-spatial references in the text it will be possible to move between text and geo-spatial database and identify information on specific locations more efficiently. This system provides the groundwork for the future interoperability of systems.

In collaboration with the Research Group Cartography of the TU Vienna we are working on semantically modeling the cartographic information on the topography of Ephesos so that we can take advantage of the information encoded in the maps and open up this documentation to further queries and knowledge extraction.

The second issue comes to play in later FiE publications, such as the standard site map illustrating the location of the archaeological sites. ${ }^{5}$ It nicely visualizes the amount of work that had been done in the 80 years since the first $\mathrm{FiE}$ publication and emphasizes the visitor path through Ephesos flanked by the many impressive reconstructions. ${ }^{6}$ The map is pragmatic: the amount of sites had become too dense so that the names could no longer be directly inserted into the map and instead numbers marked the sites. This map illustrates another issue of dealing with maps: they simply document the state of knowledge at the time of production of the map and are suggestive of ideal and neatly organized space but they do not indicate whether the monuments actually were contemporary. ${ }^{7}$ Not only is the time factor not reflected in this map but it also masks the complexity of individual locations. It is not able to show the development of a single site over centuries or millennia as would be necessary for a complex site like Ephesos.

The chronological factor will be an essential component of the project. On the one hand we will need to identify temporal terms as used by the various scholars but temporal terms only carry meaning if they are also defined. This means that it will be necessary to define the chronological terms used in the FiE through PeriodO $\mathrm{O}^{8}$ and include this in the encoding model of the texts. On the other hand we will also need to consider time in relationship to research history. An archaeological site is not only defined by the date of its creation, use, and abandonment but also by the time period when it was discovered, studied, published, reevaluated, ... We are currently investigating the possibility of modeling our data on the CIDOC-CRMarchaeo-model. ${ }^{9}$ Considering these actions not only in relationship to their geographic location but also to time will add another layer of information in reconstructing narratives of spaces and objects.

5 See the site plan in: Peter SchERRER, Ephesos. Der neue Führer, Wien 1995

6 For more information on the evolution of the archaeological site of Ephesos, see: Sabine LADSTÄTTER, Ephesos: Archäologie und Massentourismus, in: Katja Piesker u. a., Hg., Heritage in Context 2. Archäologie und Tourismus, Miras 4, Istanbul 2018, 253-288.

7 Mark Gillings / Piraye HacıgüZelLeR / Gary Lock, Hg., Re-Mapping Archaeology: Critical Perspectives, Alternative Mappings, New York (2018), 1-16.

8 http://perio.do/en/ (01.04.2019).

9 http://www.cidoc-crm.org/crmarchaeo/home-3 (01.04.2019). 
A third complication in the geo-spatial data can be identified in the modern archaeological publications. By the mid-1990s the archaeological exploration of Ephesos became more detailed in its documentation and careful excavation of sites according to layers. Modern archaeology emphasizes the relationship between finds and layer where finds were recovered leading to meticulously documentation. The documentation of layers often gives a better sense of the scrappy realities of the built environment (construction, abandonment of sites, frequency of renovations, disrepair, and unfinished construction) but these illustrations are typically detached from maps, at least in their analog published form. Merging maps with the documented layers would provide a third dimension to understanding sites not only as separate monuments but as a continually evolving network of structures embedded into the wider development of a site. This would make it possible to not only explore the history of a single location over time but by including this third dimension the data could be visualized in more stimulating ways and lead to the identification of patterns that would otherwise remain hidden.

An immediate result of the project will be the creation of digital facsimiles of the FiE for archival purposes as well as for referencing. We are also developing a workflow for the digitization and preparation of the metadata so that future publications can be added as structured data to the spatially driven archive in consistent quality. All concepts will have unique identifiers and will be included in the encoding of the digital FiE so that scholars can move freely between maps and text. Furthermore, the data will be available through secure long-term hosting and presentation of the data in a subject-specific repository. Finally, the digital editions will include annotations to geo-spatial information and the digital foundation for further geo-spatial modeling and linking with our GIS-system will be in place. Our highly ambitious long-term goal is to create a sustainable archaeological linked data network by transforming our existing databases into interoperable systems. By making the data machine readable we are ensuring that the work of our predecessors at Ephesos is not forgotten but instead that it is made available for reuse and included in the developing knowledge network. This will lead to the possibility of increasingly more complex research questions by connecting our datasets with other datasets but will also be an important aspect in order to minimize disciplinary and linguistic boundaries.

\section{Literature}

Otto BenNDORF, Hg., Forschungen in Ephesos, Wien 1906.

http://www.cidoc-crm.org/crmarchaeo/home-3 (01.04.2019).

Mark GILLINGS/Piraye HACIGÜZELLER/Gary Lock, Hg., Re-MappingArchaeology: Critical Perspectives, Alternative Mappings, New York (2018), 1-16.

Sabine LADSTÄTTER, Ephesos: Archäologie und Massentourismus, in: Katja Piesker u. a., Hg., Heritage in Context 2. Archäologie und Tourismus, Miras 4, Istanbul 2018, 253-288.

Sabine LADSTÄTTER, Hafen und Stadt von Ephesos in hellenistischer Zeit, in: Jahreshefte des Österreichischen Archäologischen Institutes 85 (2016), 233-272.

Ben MARWICK / Suzanne BIRCH, A Standard for the Scholarly Citation of Archaeological Data as an Incentive to Data Sharing, in: Advances in Archaeological Practice 6/2 (2018), 125-143.

http://perio.do/en/ (01.04.2019).

https://www.propylaeum.de/home/ (01.04.2019).

Peter ScHERRER, Ephesos. Der neue Führer, Wien 1995.

Adela SовоткоVA, Sociotechnical Obstacles to Archaeological Data Reuse, in: Advances in Archaeological Practice 6/2 (2018), 117-124. 


\title{
Überlegungen zur Digitalen Archäologie und zu ihrem Verhältnis zu den Digitalen Geisteswissen- schaften
}

\author{
Dominik Hagmann \\ Universität Wien, Institut für Klassische Archäologie \\ dominik.hagmann@univie.ac.at \\ https://homepage.univie.ac.at/dominik.hagmann
}

Keywords: Digital Archaeology, Theory, Taxonomy, Academic Fields

\begin{abstract}
What does the term 'Digital Archeology' exactly mean and how does it relate to the Digital Humanities? The paper wants to take up as well as critically address just that question and gives a short outlook on further considerations.
\end{abstract}

\section{Fragestellung}

Was kann der Begriff „Digitale Archäologie“ (DA) genau bedeuten und in welcher Beziehung steht er zu den "Digitalen Geisteswissenschaften" (DG)? Der nachfolgende Beitrag will diese Frage aufgreifen und kritisch behandeln sowie einen kurzen Ausblick auf weitere Überlegungen geben.

\section{Versuch einer Charakterisierung der „Digitalen Archäologie“}

„Archäologie“ widmet sich der Erforschung der materiellen Kultur vergangener Zeiten ${ }^{1}$, unter der Verwendung verschiedenster Methoden und Theorien. ${ }^{2}$ Bei „Archäologie“ handelt es sich jedoch nicht um ein einziges Fach - vielmehr ist eine eindeutige Charakterisierung kaum möglich, da eine Vielfalt an speziellen (interdisziplinären) Ausrichtungen ${ }^{3}$ innerhalb des Faches auszumachen ist. So gliedert sich „Archäologie“ in teilweise vollkommen unabhängige Teilfächer, die ihrerseits von verschiedenen Forschungstraditionen geprägt sind. Diese Vielfalt ist dabei inklusiv und die Grenzen sind nicht eindeutig: Kulturwissenschaftliche Analysen sind ebenso üblich wie bioarchäologische Studien von Tierresten zur Rekonstruktion des Ernährungsverhaltens.

Die beschriebene Vielfalt kann wohl als einer der Gründe angesehen werden, dass es bereits unmittelbar nach dem Aufkommen computergestützter Verfahren zu ersten Gehversuchen im Bereich datengetriebener archäologischer Studien kam. ${ }^{4}$ Seit der Verfügbarkeit von digitalen Informations-

1 Vgl. zu einer Definition etwa Mikkel Bitle / Tim Flohr Sørensen, Material Culture, in: Sandra L. López Varela, Hg., The Encyclopedia of Archaeological Sciences, Malden 2018, 1-5.

2 Jane BALME, Artifacts, Overview, in: Deborah Marie Pearsall, Hg., Encyclopedia of Archaeology, San Diego 2008, 508517, hier 509; Manfred K. H. EgGERT, Prähistorische Archäologie. Konzepte und Methoden, 4. Auflage, Tübingen 2012; Tonio HöLSCHER, Klassische Archäologie. Grundwissen, 4. Auflage, Darmstadt 2015; Vgl. hierzu etwa Colin RENFREW / Paul G. BAHN, Archaeology. Theories, Methods and Practice, 7. Auflage, London 2016.

3 Im Folgenden als Teildisziplinen, -fächer und dergleichen bezeichnet.

4 Ethan Watrall, Archaeology, the Digital Humanities, and the "Big Tent”, in: Matthew K. Gold / Lauren F. Klein, Hg., Debates in the Digital Humanities 2016, Minneapolis 2016, 345-358, hier 346-349. 
und Kommunikationstechnologien (IKT) werden diese ebenso für archäologische Fragestellungen herangezogen, anfangs besonders um mit modernen Methoden effektiv große Datenmengen zu erzeugen und zu verarbeiten. ${ }^{5}$ Dieser Umstand wird von P. T. Daly und T. L. Evans in ihrem Standardwerk zur DA aus dem Jahr 2006 eindeutig beschrieben: „Digital Archaeology explores the basic relationships that archaeologists have with Information and Communication Technology (ICT) and digital technology to assess the impact that such innovations have had on the very basic ways that archaeology is performed and considered." Nach über 50 Jahren an archäologischer Arbeit in diesem Gebiet kann diese Definition einer „Fusion“ von Informatik und Archäologie(n) um weitere Merkmale ergänzt werden, wie etwa um die Rolle der digitalen Public Archaeology als inklusives Element zwischen Wissenschaft und Gesellschaft im Bereich der DA. ${ }^{7}$ Auch muss die Ubiquität digitaler Methoden ${ }^{8}$ berücksichtigt werden oftmals wird deren Einsatz bereits als gegeben vorausgesetzt und nicht (mehr) als explizites Merkmal der DA gesehen. ${ }^{9}$ Demnach obliegt es der DA, nicht "nur“ die bloße Verwendung von digitalen Tools zu behandel $\mathrm{n}^{10}$, sondern vielmehr auch die theoretische Auseinandersetzung mit der Materie zu forcieren: „All archaeologies can be digital, but not all archaeologies are Digital Archaeology.“11

Der Umstand, verschiedene IKT zur Erforschung der materiellen Kultur praktisch einzusetzen und auch theoretisch zu behandeln, wird mit verschiedenen Termini wie „DA“12, „Virtuelle Archäologie“13 oder "Cyber-Archäologie"14 beschrieben. Diese verfügen jeweils über konkrete Ausprägungen und gegebenenfalls auch bestimmte Abhängigkeiten zueinander. ${ }^{15}$ Nicht zuletzt kann zudem festgestellt werden, dass einzelne Begriffe zu gewissen Zeitpunkten als trending terms besonders in Mode sind. ${ }^{16}$

Doch was meint der Begriff „DA“, der hier als Platzhalter für die diversen Termini Verwendung findet, eigentlich? Bei genauerer Betrachtung stellt sich schnell die Frage, ob sich kongruent zur jeweiligen Fachrichtung (z. B. Prähistorische Archäologie, Klassische Archäologie etc.) mit „DA“ jeweils eine eigene digitale Teildisziplin etabliert. Muss in weiterer Folge also im Grunde von „Digitaler Prähistorischer“ und „Digitaler Klassischer Archäologie“ gesprochen werden und handelt es sich dabei um Schwesternwissenschaften? Sollen diese Teilfächer dann in einem weiteren Schritt unabhängig voneinander institutionalisiert oder in einer selbstständigen Disziplin „DA“ subsummiert werden? Übernimmt diese DA (womöglich auch als Institution) danach alle wissenschaftlichen und lehrbezogenen Zuständigkeiten?

5 Shawn Graham u. a., The Open Digital Archaeology Textbook 2019-ongoing, online unter: https://o-date.github.io/draft/ book/so-what-is-digital-archaeology.html\#a-distant-view (12.03.2019); Patrick T. DALY / Thomas L. Evans, Introduction. Archaeological Theory and Digital Pasts, in: Thomas L. Evans / Patrick T. Daly, Hg., Digital Archaeology: Bridging Method and Theory, London u.a. 2006, 2-7.

6 DALY / EVANS, Introduction, 2.

7 Graham, Open; Dominik Hagmann, Reflections on the Use of Social Networking Sites as an Interactive Tool for Data Dissemination in Digital Archaeology, in: Interdisciplinaria Archaeologica 9/1 (2018), 7-20; Lorna-Jane RICHARDSON / Jaime AlmANSA-SÁnchez, Do You Even Know What Public Archaeology Is? Trends, Theory, Practice, Ethics, in: World Archaeology 47/2 (2015), 194-211; Lorna-Jane RICHARDSON, Ethical Challenges in Digital Public Archaeology, in: Journal of Computer Applications in Archaeology 1/1 (2018), 64-73.

8 William R. CARAHER, Continuity, Practice, and Theory in Digital Archaeology, online unter: https://mediterraneanworld.wordpress.com/2018/12/17/continuity-practice-and-theory-in-digital-archaeology/ (24.01.2019).

9 Andre Costopoulos, Digital Archeology Is Here (and Has Been for a While), in: Frontiers in Digital Humanities 3 (2016).

10 Graham, Open.

11 Ebd.

12 Ezra B. W. ZuBRow, Digital Archaeology. A Historical Context, in: Thomas L. Evans / Patrick T. Daly, Hg., Digital Archaeology: Bridging Method and Theory, London u.a. 2006, 8-26.

13 Paul ReILly, Towards a Virtual Archaeology, in: Sebastian Rahtz / Kris Lockyear, Hg., CAAgo: Computer Applications and Quantitative Methods in Archaeology 1990, Oxford 1991, 132-139.

14 Thomas Evan LeVY u. a., Cyber-Archaeology in the Holy Land. The Future of the Past, San Diego 2012.

15 Ebd., 4.

16 Jeremy HuggetT, Core or Periphery? Digital Humanities from an Archaeological Perspective, in: Historical Social Research: Historische Sozialforschung 37/3 (2012), 86-105, hier 90. 
Oder handelt es sich bei DA eher um die Bezeichnung eines „Phänomens“, das zwar die Anwendung von IKT in der Archäologie in Theorie und Praxis beschreibt, aber jedem einzelnen Teilfach im Rahmen der „regulären“ archäologischen Arbeit ohnehin ständig innewohnend ist? Könnte dies in weitere Folge also heißen, dass es sich bei DA zwar um die Auseinandersetzung mit IKT innerhalb eines archäologischen Fachbereichs handelt, diese sich jedoch nicht zu einem dezidierten Fach, sondern zu einer Metadisziplin ausformt? ${ }^{17}$

\section{Das Verhältnis von DA und DG}

Die fachliche Variabilität der Archäologie ist das Hauptproblem der taxonomischen Verortung der Beziehung der DA und DG zueinander, denn die archäologische Disziplinenlandschaft wird keineswegs ausschließlich den Geisteswissenschaften zugeordnet - besonders im anglo-amerikanischen Raum wird Archäologie oftmals als Sozialwissenschaft betrachtet. ${ }^{18}$ In Mitteleuropa werden hingegen in taxonomischer Hinsicht die „Archäologien“ meist den Geisteswissenschaften zugeteilt. ${ }^{19}$

Betrachtet man Archäologie nun als Geisteswissenschaft, kann rein taxonomisch auch eine Verbindung zwischen den jeweiligen digitalen Bereichen hergestellt werden. In Anbetracht der zuvor angestellten Überlegungen gilt es hierfür unmittelbar in einem weiteren Schritt zu klären, was genau diese digitalen Bereiche in beiden Fällen darstellen sollen: spezialisiertes Arbeiten innerhalb eines Teilfachs oder selbstständige Fächer? Handelt es sich dann bei DA um eine Subdisziplin der DG ${ }^{20}$ Oder bestehen DA und DG dennoch ohne Verbindung nebeneinander? Definiert man Archäologie hingegen als sozialwissenschaftlich orientiertes Fach, stellt sich die Frage, ob sich dementsprechend auch die Beziehung der DA zur DG ändert. Sind in diesem Fall DG und DA zwar strukturell gesehen vergleichbar, aber nicht voneinander abhängig, wie es besonders von sozialwissenschaftlich orientierten Archäolog* innen gesehen wird ${ }^{21}$ ?

\section{Offene Fragen und Ausblick}

Was ist also DA? Diese Frage kann hier vorerst nicht beantwortet werden. Die DA fungiert als Schnittstelle für alle Aspekte der Digitalisierung zwischen diversen archäologischen Teilfächern und kann als Spezialisierung gelten. ${ }^{22}$ DA bietet die Möglichkeit, digitale Methoden auszuwählen, fachgerecht umzusetzen und dies praktisch sowie theoretisch zu evaluieren. Weniger scheint man DA hingegen als eine Fachdisziplin umreißen, einem Fach zuordnen oder einer Forschungstradition zuweisen zu können. Zu beachten ist, dass die Entwicklung der DA trotz allem nach wie vor erst am Anfang steht.

Nur ihre langfristige Beobachtung kann womöglich Aufschluss über die offenen Fragen geben. ${ }^{23}$ Die Frage nach dem genauen Verhältnis von DA und DG konnte hier ebenso vorerst nicht eindeutig

17 Hagmann, Reflections, gf.

18 WATRALL, Archaeology, 349f.

19 Statistik Austria, Österreichische Systematik der Wissenschaftszweige (ÖFOS) 2012, online unter: https://www.data.gv.at/ katalog/dataset/stat_ofos-2012 (24.04.2019).

20 Vgl. hierzu das 3-Sphären-Modell zur Kartierung der Digital Humanities in Patrick SAHLE, Digital Humanities? Gibt's doch gar nicht!, in: Sonderband der Zeitschrift für digitale Geisteswissenschaften 1 (2015), hier 7.

21 WATRALL, Archaeology.

22 Katy MEYERS, Defining Digital Archaeology, online unter: http://chi.anthropology.msu.edu/2011/10/defining-digital-archaeology/ (12.03.2019).

23 Erin Walcek Averett / Jody Michael Gordon / Derek B. CounTs, Mobile Computing in Archaeology. Exploring and Interpreting Current Practices, in: Erin Walcek Averett u. a., Hg., Mobilizing the Past for a Digital Future: The Potential of Digital Archaeology, Grand Forks 2016, 1-30, hier 9. 
beantwortet werden. Es muss weiterführend gefragt werden, ob eine präzise taxonomische Verortung tatsächlich der richtige Ansatz ist, denn je nach Forschungstradition und genauer fachlicher Ausrichtung kommt es zur Anwendung unterschiedlicher Systematiken und damit zu unterschiedlichen Ergebnissen - eine pauschale Antwort ist jedenfalls nicht möglich.

Unabhängig von der angerissenen Problematik besitzen die DG jedenfalls das Potential als „Brücke“ zwischen den digital betriebenen Archäologien und Geisteswissenschaften zu dienen. ${ }^{24}$ Bestes Beispiel für eine praktikable Umsetzung dieses Gedankens sind Tagungen wie die DHA selbst, die eine Plattform für eine derartige Vernetzung darstellen und diese ebenso fördern.

\section{Literaturverzeichnis}

Erin Walcek Averett / Jody Michael GoRdon / Derek B. Counts, Mobile Computing in Archaeology. Exploring and Interpreting Current Practices, in: Erin Walcek Averett u. a., Hg., Mobilizing the Past for a Digital Future: The Potential of Digital Archaeology, Grand Forks 2016, 1-30.

Jane BALME, Artifacts, Overview, in: Deborah Marie Pearsall, Hg., Encyclopedia of Archaeology, San Diego 2008, 508-517.

Mikkel BILLE / Tim Flohr Sørensen, Material Culture, in: Sandra L. López Varela, Hg., The Encyclopedia of Archaeological Sciences, Malden 2018, 1-5.

William R. CARAHER, Continuity, Practice, and Theory in Digital Archaeology, online unter: https://mediterraneanworld.wordpress.com/2018/12/17/continuity-practice-and-theory-in-digital-archaeology/ (24.01.2019).

Andre Costopoulos, Digital Archeology Is Here (and Has Been for a While), in: Frontiers in Digital Humanities 3 (2016), DOI: 10.3389/fdigh.2016.00004.

Patrick T. DaLY / Thomas L. Evans, Introduction. Archaeological Theory and Digital Pasts, in: Thomas L. Evans / Patrick T. Daly, Hg., Digital Archaeology: Bridging Method and Theory, London u.a. 2006, 2-7.

Manfred K. H. EgGert, Prähistorische Archäologie. Konzepte und Methoden, 4. Auflage, Tübingen 2012.

Shawn Graham u. a., The Open Digital Archaeology Textbook 2019-ongoing, online unter: https://o-date.github. io/draft/book/so-what-is-digital-archaeology.html\#a-distant-view (12.03.2019).

Dominik HagmanN, Reflections on the Use of Social Networking Sites as an Interactive Tool for Data Dissemination in Digital Archaeology, in: Interdisciplinaria Archaeologica 9/1 (2018), 7-20.

Tonio HöLsCHER, Klassische Archäologie. Grundwissen, 4. Auflage, Darmstadt 2015.

Jeremy HuggetT, Core or Periphery? Digital Humanities from an Archaeological Perspective, in: Historical Social Research: Historische Sozialforschung 37/3 (2012), 86-105.

Thomas Evan LeVY u. a., Cyber-Archaeology in the Holy Land. The Future of the Past, San Diego 2012.

Katy MeYERS, Defining Digital Archaeology, online unter: http://chi.anthropology.msu.edu/2011/10/defining-digital-archaeology/ (12.03.2019).

Paul ReILly, Towards a Virtual Archaeology, in: Sebastian Rahtz / Kris Lockyear, Hg., CAA90: Computer Applications and Quantitative Methods in Archaeology 1990, Oxford 1991, 132-139.

Colin Renfrew / Paul G. BAhn, Archaeology. Theories, Methods and Practice, 7. Auflage, London 2016.

Lorna-Jane RichARDSON, Ethical Challenges in Digital Public Archaeology, in: Journal of Computer Applications in Archaeology 1/1 (2018), 64-73.

Lorna-Jane RICHARDSON / JaimeAlmANSA-SÁNCHEZ, Do You Even Know What PublicArchaeology Is? Trends, Theory, Practice, Ethics, in: World Archaeology 47/2 (2015), 194-211.

24 HUgGetT, Core, 101. 
Patrick SAHLE, Digital Humanities? Gibt's doch gar nicht!, in: Sonderband der Zeitschrift für digitale Geisteswissenschaften 1 (2015), DOI: 10.17175/sb001_004.

Statistik Austria, Österreichische Systematik der Wissenschaftszweige (ÖFOS) 2012, online unter: https://www. data.gv.at/katalog/dataset/stat_ofos-2012 (24.04.2019).

Ethan WATRALL, Archaeology, the Digital Humanities, and the "Big Tent", in: Matthew K. Gold / Lauren F. Klein, Hg., Debates in the Digital Humanities 2016, Minneapolis 2016, 345-358.

Ezra B. W. ZuBrow, Digital Archaeology. A Historical Context, in: Thomas L. Evans / Patrick T. Daly, Hg., Digital Archaeology: Bridging Method and Theory, London u.a. 2006, 8-26. 


\title{
Development of a Cross-Project Citizen Science Platform for the Humanities
}

\author{
Hendrikje Carius [1], Christiane Kuller [2], Patrick Rössler [3] \& René Smolarski [4]
}

[1] Gotha Research Library of the University of Erfurt

hendrikje.carius@uni-erfurt.de

https://orcid.org/0000-0002-8413-2355

[2] Chair of Modern and Contemporary History and History Didactics, University of Erfurt christiane.kuller@uni-erfurt.de

[3] Chair of Communication Science with the focus on Empirical Communication Research / Methods, University of Erfurt

patrick.roessler@uni-erfurt.de

[4] Modern and Contemporary History and History Didactics, University of Erfurt

rene.smolarski@uni-erfurt.de

https://orcid.org/0000-0001-8854-4341

Keywords: Citizen Science, Virtual Research Infrastructure, Public History, Cinema History, History of Everyday Life in the GDR

\begin{abstract}
This article presents a project at the University of Erfurt to establish a Citizen Science platform on the issue of GDR Cinema History. Public interest assumed for this topic will be activated by both analogous and digital communication, contributions collected online and integrated into the research process.
\end{abstract}

Genuine digital approaches have become firmly established components of Citizen Science or Crowdsourcing projects. In the course of the public and digital turn in research and the knowledge society, they contribute to a linkage between science and the public. This opens up fresh opportunities for an approach, which, in the sense of a broad definition of Citizen Science, includes the dialogue with an interested public as an integral part of a research process that expands beyond the unidirectional information and education function and rather relies on dialogue-oriented and participatory formats. ${ }^{1}$

Citizen Science has been established in Germany through initiatives such as the GEWISS consortium Bürger schaffen Wissen (Citizens Create Knowledge), ${ }^{2}$ primarily in the natural sciences, and has been

1 For Citizen Science in the humanities see among others: Kristin OswaLD / René SMOLARSKI, Ed., Bürger Künste Wissenschaft. Citizen Science in Kultur und Geisteswissenschaften, Gutenberg 2016; Cord ARENDES, Historiker als „Mittler zwischen den Welten“? Produktion, Vermittlung und Rezeption historischen Wissens im Zeichen von Citizen Science und Open Science, in: Heidelberg Jahrbücher Online 2 (2017), 19-58.

2 The consortium is funded by the German Federal Ministry of Education and Research (BMBF) and coordinated by the Museum für Naturkunde Berlin - Leibniz Institute for Evolutionary and Biodiversity Science (MfN) and the Helmholtz Centre for Environmental Research (UFZ). 
tested in numerous projects. ${ }^{3}$ Since then, a number of successful projects have also been implemented in the humanities and social sciences. These include, for example, those that aim to involve Citizen Scientists in the creation of transcriptions, such as Transcribe Bentham, Transcribe Europeana 19141918, e-manuscripta and Letters $1916-1923^{4}$, or projects to identify or collect objects such as Artigo. ${ }^{5}$

The existing digital infrastructures for Citizen Science projects in the humanities are usually oriented towards individual projects and data acquisition and do not offer a structure that works across different projects which thus remains a desideratum so far. This is the starting point for an interdisciplinary and infrastructural project to set up a Citizen Science platform at the University of Erfurt. ${ }^{6}$ Based on an issue, which is relevant for everyday history in the GDR, we will lay an infrastructural basis for systematic cooperation between citizens and academic institutions, test new concepts for the integration of Citizen Science into academia, reflected from the perspectives of information, history and communication studies. The concept of Citizen Science is understood in a broad sense as the participation of the (non-specialist) public in various phases of research processes in compliance with academic standards. ${ }^{7}$

The project is designed as an interdisciplinary cooperation and based on two research units: the Network for Digital Humanities and Citizen Science and the Interdisciplinary Research Centre for Historical Media at the University of Erfurt, supported by the Chairs of Modern and Contemporary History and History Didactics as well as the Chair of Communication Studies with a focus on Empirical Communication Research / Methods. In the field of IT, the project cooperates with the Technical University of IImenau, with the Gotha Research Library of the University of Erfurt representing another partner during the stage of conception. The project will use and evaluate established solutions to develop generic, sustainable infrastructures in order to build a technical foundation for different Citizen Science initiatives within the modular digital research environment at the University of Erfurt. ${ }^{8}$

Based on a concrete case study entitled "Kino in der DDR - Rezeptionsgeschichte ,von unten"' (Cinema in the GDR - a bottom-up History of Media Use), we address the public's interest in the humanities and social sciences. People are activated for university research using digital methods; they are connected and productively integrated into the academic work process. ${ }^{9}$ Non-university citizens can participate in various panels and in different stages of the research process (data collection, processing and evaluation). Their activities include a broad spectrum of image and text classifications, transcription and annotation work, and other forms of evaluation of historical collections and data.

3 An overview of international Citizen Science projects can be found on Wikipedia: List of citizen science projects, online unter: https://en.wikipedia.org/wiki/List_of_citizen_science_projects (30.03.2019).

4 Transcribe Bentham, online unter: https:///blogs.ucl.ac.uk/transcribe-bentham/; Transcribathon, online unter: https://transcribathon.com/en/; e-manuscripta, online unter: https://www.e-manuscripta.ch/; Letters 1916-1923, online unter: http:// letters1916.maynoothuniversity.ie/ (all 30.03.2019).

5 Artigo, online unter. https://www.artigo.org/ (30.03.2019).

6 The project "Development of a Citizen Science Platform to Involve the Public in the Scientific Work Process at the University Erfurt" will be funded by the Thüringer Aufbaubank in 2019-2022.

7 Lisa PettiBone et al., Ed., Citizen Science für alle. Eine Handreichung für Citizen Science Beteiligte, Berlin $2016,6$.

8 One example is the Gotha Portal, which is being created as part of the Sammlungs- und Forschungsverbund Gotha (Research Library and Research Centre of the University of Erfurt, Foundation Castle Friedenstein Gotha) and unites the resources of the research location on digital grounds. Interfaces are to be implemented between the planned research environment and the Citizen Science Portal for worldwide research with historical objects and collections. See: Forschen in Gotha, online unter: https://www.gotha3.de/forschungsblog/forschen-in-gotha/gotha3/gotha-portal (30.03.2019).

9 See knowledge transfer formats such as Science Slams, Long Nights of the Sciences/Museums or Science Café as well as the participation of citizens in relevant topics such as numismatics, philately or genealogy. Cooperation is also planned with "Bürger schaffen Wissen" and other international communities. 


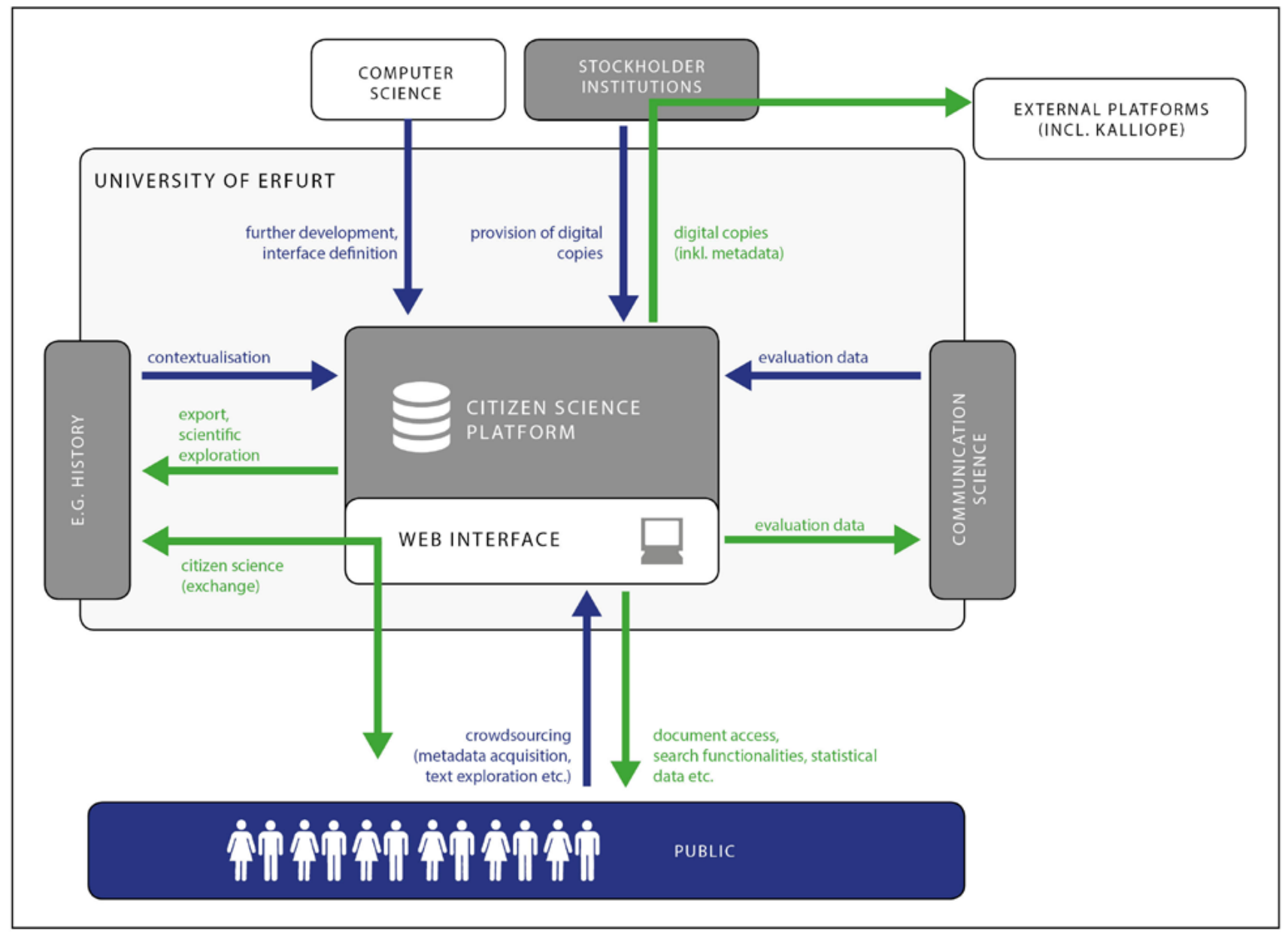

Fig. 1: Overall structure of the project

Methodologically, approaches of Digital Humanities and Citizen Science as well as from communications, computer science and history are intertwined. Our multi-perspective approach combines the analysis of the functional conditions of Citizen Science communication with the informational and technological conception and the realization of the platform. This also includes the use of elements typically used for computer games to increase motivation (gamification). In the pilot project, conceptual questions concerning the process (cooperation, interaction and negotiation of roles between the individual actors; community building and management; user expectations and recognition culture), data curation, quality assurance as well as the conditions of the legal and ethical framework will be reflected.

Especially in the field of GDR history, instruments which facilitate the exchange between university research and non-academic debates still are a desideratum. Thus far, the film history of the GDR has been written primarily as a history of film politics, ${ }^{10}$ content analysis ${ }^{11}$ or production history, ${ }^{12}$ while a historical account detailing the reception of these films is still lacking in many respects. Using Citizen Science and Crowdsourcing approaches, the project thus attempts for the first time to reconstruct the appropriation of movies based on narratives and documents from the private sphere, in order to close the existing source gap.

\footnotetext{
10 Dagmar SchITtLY, Zwischen Regie und Regime. Die Filmpolitik der SED im Spiegel der DEFA-Produktionen, Berlin 2002.

11 Klaus FINKE, Politik und Film in der DDR, Oldenburg 2007.

12 Günter JORDAN, Film in der DDR. Daten, Fakten, Strukturen, Potsdam 2009.
} 
The unique holdings of the Interdisciplinary Research Centre for Historical Media at the University of Erfurt (IRCHM) on cinema films in the GDR form one of the sources for the pilot project. The IRCHM has around 150,000 individual objects at its disposal, including film posters, stills, press photos and other advertising material. The collection covers almost all films screened in the GDR since the mid1950 s, i.e. the holdings consist not only of the GDR's own DEFA productions, but as well of all productions from eastern and western countries permitted for screening via the central Progress Film-Verleih (film distribution), plus materials on those films banned by the GDR government's censorship.

On the one hand, the platform is intended as an opportunity of, in the sense of a crowdsourcing approach, sharing self-experienced stories and additional source material via upload. This provides new information and sources related to the everyday history in the GDR that could not be obtained otherwise since most of this evidence can only be retrieved from contemporary witnesses. However, traditional methods of data collection would be far too extensive in this realm. Moreover, the narratives prevalent in society add specific evidence when the legitimacy and recognition of different narratives is being fought over currently in a particularly conflictual manner. Reflecting on the dialogue between academia and public, the Citizen Science platform will generate benefit for the digital-based Citizen Science and its methodology in general, as well as for the associated fields such as gamification, e-learning and media studies.

\section{Literature}

Cord ARENDES, Historiker als „Mittler zwischen den Welten“? Produktion, Vermittlung und Rezeption historischen Wissens im Zeichen von Citizen Science und Open Science, in: Heidelberg Jahrbücher Online 2 (2017), 19-58.

Artigo, online unter: https://www.artigo.org/ (30.03.2019).

e-manuscripta, online unter: https://www.e-manuscripta.ch/ (30.03.2019).

Klaus FINKE, Politik und Film in der DDR, Oldenburg 2007.

Forschen in Gotha, online unter: https://www.gotha3.de/forschungsblog/forschen-in-gotha/gotha3/gotha-portal (30.03.2019).

Günter JORDAN, Film in der DDR. Daten, Fakten, Strukturen, Potsdam 2009.

Letters 1916-1923, online unter: http://letters1916.maynoothuniversity.ie/ (30.03.2019).

List of citizen science projects, online unter: https://en.wikipedia.org/wiki/List_of_citizen_science_projects (30.03.2019).

Kristin OsWALD / René SMOLARSKI, Ed., Bürger Künste Wissenschaft. Citizen Science in Kultur und Geisteswissenschaften, Gutenberg 2016.

Lisa Pettibone et al., Ed., Citizen Science für alle. Eine Handreichung für Citizen Science Beteiligte, Berlin 2016.

Dagmar SCHITTLY, Zwischen Regie und Regime. Die Filmpolitik der SED im Spiegel der DEFA-Produktionen, Berlin 2002.

Transcribathon, online unter: https://transcribathon.com/en/ (30.03.2019).

Transcribe Bentham, online unter: https://blogs.ucl.ac.uk/transcribe-bentham/ (30.03.2019). 


\section{Die Edition der Ministerrats- protokolle 1848-1918 digital}

Workflows, Möglichkeiten, Grenzen

Stephan Kurz, Wladimir Fischer-Nebmaier, Dario Kampkaspar, Richard Lein, Anatol Schmied-Kowarzik

Österreichische Akademie der Wissenschaften stephan.kurz@oeaw.ac.at, https://orcid.org/0000-0003-2546-2570

wladimir.fischer-nebmaier@oeaw.ac.at, https://orcid.org/0000-0002-6725-9012 dario.kampkaspar@oeaw.ac.at, https://orcid.org/0000-0002-0118-0811 richard.lein@oeaw.ac.at, https://orcid.org/0000-0002-7502-0503 anatol.schmied-kowarzik@oeaw.ac.at, https://orcid.org/0000-0002-4113-4713 https://www.oeaw.ac.at/inz/digitales/ und https://www.acdh.oeaw.ac.at/

Keywords: Historical Administrative Sources, Digital Edition, Hybrid Edition, Auxiliary Data, Linked Open Data, Text Encoding Initiative (TEI)

Abstract: A long-term edition project that aims to publicise historical records from the administrative domain is currently undergoing a transformation of its edition workflow. The paper discusses necessary prerequisites, conceptual changes and shifting demands toward historical source editions by way of the Austrian Academy of Sciences' 'Ministerratsprotokolle Österreichs und der Österreichisch-Ungarischen Monarchie 1848-1918' edition.

\section{Quellen und Material}

Seit 1970 gibt es die Edition „Ministerratsprotokolle Österreichs und der Österreichisch-Ungarischen Monarchie 1848-1918“ als gedrucktes Werk. Sie steht im Range eines Langzeitvorhabens der Österreichischen Akademie der Wissenschaften und wird aktuell auf eine XML-basierte digitale Edition mit hybridem Charakter (Druck- und Onlinefassung) umgestellt.

Ediert wird eine zentrale historische Quelle des gouvernementalen Systems der Monarchie. Es geht darum, die handschriftlichen Originale zuverlässig abzuschreiben und mit Hilfe von ergänzendem Aktenmaterial und Sekundärliteratur wissenschaftlich-kritisch zu kommentieren. Dazu ist sorgfältige Archivarbeit mit dem reichen Originalaktenbestand der Ministerien und der Kabinettskanzlei nötig.

Der Ministerrat war das zentrale Organ der Regierungstätigkeit in der Habsburgermonarchie beziehungsweise nach dem „Ausgleich“ 1867 im cisleithanischen Teil Österreich-Ungarns. Seine Sitzungsprotokolle präsentieren alle Facetten staatlichen Lebens, von Fragen der Struktur und der Organisation des Staates bis zu gesellschaftlichen, wirtschaftlichen und technischen Entwicklungen sowie 
kulturellen und sozialen Problemen. Die Edition der Ministerratsprotokolle, die seit über 40 Jahren unter wechselnder editorischer Verantwortung erscheint, hat bislang 28 gedruckte Bände mit rund 18.000 Druckseiten vorzuweisen. Nun wird aktuell am Forschungsbereich Kulturelles Erbe des Instituts für Neuzeit- und Zeitgeschichtsforschung an der Österreichischen Akademie der Wissenschaften auf ein TEl-basiertes hybrides Editionsverfahren umgestellt. Hiermit betritt das Editionteam insofern Neuland, als bisher nur wenige historische Quelleneditionen - noch weniger aus der speziellen Textsorte Protokoll - aus dem administrativen Bereich bzw. aus dem 19. Jahrhundert vorliegen.

Die wissenschaftliche Edition der Ministerratsprotokolle ist aus mehreren Gründen eine besondere Edition:

- Der Umfang der zu edierenden Quellen ist groß, denn

» die abzudeckende Zeitspanne beträgt 60 Jahre,

» die textuelle Varianz etwa in den Schreibungen ist beträchtlich und

» dies hat etwa für die Textkritik, aber auch für die Arbeit mit Named Entities Konsequenzen.

- Für den Editionszeitraum 1867-1918 (3. Serie) kommt die unterschiedliche Provenienz der Quellen hinzu, denn es handelt sich

» teilweise um durch den Justizpalastbrand 1927 beschädigte oder vernichtete Protokolle („Brandakten“), die ergänzt werden,

" diese ergänzenden Quellen stammen aus verschiedenen Archivbeständen innerhalb und außerhalb des Österreichischen Staatsarchivs.

- Es gibt vielfältige interne und externe Bezüge

» zwischen Tagesordnungspunkten und Sitzungen, aber auch z.B. bandübergreifend,

» zwischen ediertem Text und wissenschaftlichem Kommentar,

» zu den „Registern“ und Verzeichnissen.

\section{Vom Print zum Digitalen}

Seit 1971 konnten unter wechselnder Herausgeberschaft 28 Bände der 1. Serie (Ministerrat 18481867) vorgelegt werden; ${ }^{1}$ sie betreffen die Arbeit der Regierungen seit der Einrichtung des Ministerrats zu Beginn des Neoabsolutismus bis zur staatlichen Neuorganisation infolge des ÖsterreichischUngarischen Ausgleichs. Die späte Habsburgermonarchie ab 1867 hatte drei Regierungen: (1) Der sogenannte "gemeinsame Ministerrat" behandelte die gemeinsamen Agenden Österreich-Ungarns (Außenpolitik, Militär und Finanzen); seine Protokolle ediert die Ungarische Akademie der Wissenschaften als 2. Serie, aus der 6 Teilbände bereits vorliegen. (2) Der ungarische Ministerrat war für das Königreich Ungarn zuständig. (3) Das ranghöchste Organ der „österreichischen Reichshälfte“ war der cisleithanische Ministerrat: Seine Protokolle sind der Editionsgegenstand der nun entstehenden 3. Serie.

$1 \quad$ Zu den Grundsätzen und Zielen der Edition vgl. Helmut RuMPLER, Hg., Die Ministerratsprotokolle Österreichs und der Österreichisch-Ungarischen Monarchie 1848-1918, Ser. 1: Die Protokolle des Österreichischen Ministerrates 1848-1867, Einl.-Bd.: Ministerrat und Ministerratsprotokolle 1848-1867. Behördengeschichtliche und aktenkundliche Analyse, Wien 1970. 
Die beiden wissenschaftlichen Haupterträge der Edition sind erstens die Protokolltexte in normalisierter und modernisierter Schreibung sowie zweitens eine ausführliche Einleitung zum jeweiligen Berichtszeitraum. Daneben tragen zur Erschließung der historischen Dokumente als Quellen für die Forschung umfangreiche Paratexte bei: in Form von Listen der Teilnehmer am Ministerrat, Verzeichnissen der verwendeten Abkürzungen und veralteter Ausdrücke.

Der wichtigste Bestandteil des Erschließungsapparats jedes Bandes ist ein Register, das genannte und handelnde Personen, Ortsnamen in verschiedener Sprache und Schreibung, Institutionen und Sachschlagworte auffindbar macht.

Diese Elemente sollen auch in den mithilfe digitaler Methoden erstellten Bänden der 3. Serie weiter integraler Bestandteil der Edition bleiben, wobei die unten genannten Vorteile der hybriden Erscheinungsweise im Druck und online genutzt werden sollen.

Als Vorstufe zu der in Arbeit befindlichen digitalen Edition wurden in den Jahren 2017 und 2018 alle 28 bereits gedruckten Bände retrodigitalisiert und als PDF-Dokumente unter https://hw.oeaw.ac.at/ ministerrat/ veröffentlicht.

\section{Ziele der digitalen Edition}

Die Arbeit an der digitalen Edition orientiert sich an der Definition von Sahle:

Eine digitale Edition ist dadurch bestimmt, dass sie die allgemeinen Anforderungen an eine wissenschaftliche Edition durch die Berücksichtigung der gegenwärtigen technischen Möglichkeiten und ihrer methodischen Implikationen erfüllt. Sie folgt einem „digitalen Paradigma“. ${ }^{2}$

In Abgrenzung zu den Voraussetzungen der bestehenden Edition folgen aus dem digitalen Paradigma neue Funktionalitäten, die die Mediengebundenheit der Edition hervorstreichen und auf eine Irreduzibilität hinweisen:

Eine digitale Edition ist dadurch bestimmt, dass sie nicht ohne wesentliche Informations- und Funktionsverluste in eine typografische Form gebracht werden kann - und in diesem Sinne über die druckbare Edition hinausgeht. ${ }^{3}$

Konkret bedeutet das Arbeiten mit digitalen Methoden in der Editorik eine

Anreicherung des Textes und damit des im Prozess der Digitalisierung entstehenden digitalen Objektes mit (normierten) Metadaten, bezogen auf verschiedene Beschreibungsebenen, wie logische Textstruktur, Interpretations- oder Erzählebenen, Morphologie, Syntax u.v.m. [setze ein: Personen, Orte, Institutionen, Ereignisse..., Anm. d. Verf.]. Dabei geht es sowohl um die Sichtbarmachung der dem Artefakt immanenten Semantiken als auch um die Anreicherung der Textbasis mit Bedeutungen, die aus Interpretations- und Kontextualisierungsakten im Forschungsprozess entstehen. ${ }^{4}$

2 Patrick SAHLE, Digitale Editionsformen. Zum Umgang mit der Überlieferung unter den Bedingungen des Medienwandels, Teil 2: Befunde, Theorie und Methodik [Finale Print-Fassung], Norderstedt 2013 (Schriften des Instituts für Dokumentologie und Editorik Bd. 8), 148; Vgl. auch Patrick SAHLE, Zwischen Mediengebundenheit und Transmedialisierung. Anmerkungen zum Verhältnis von Edition und Medien, in: editio 24 (2010), 23-36, hier 24: „Eine digitale Edition ist eine Edition, die einem digitalen Paradigma folgt."

3 SAHLE, Editionsformen, Teil 2, 149.

4 Johannes StIGLER, Anmerkungen zu einem generischen Verständnis des Begriffes „Digitale Edition“, in: Anne Bohnenkamp-Renken / Elke Richter, Hg., Brief-Edition im digitalen Zeitalter, Berlin 2013 (Beihefte zu editio 34), 43-52, hier 43. 
Die digitale Edition der Ministerratsprotokolle geht in diesem Sinne über die Funktionalitäten der bisher erschienenen Bände hinaus; eine Druckvorlage wird im gewählten „hybriden“ Editionsansatz dennoch erzeugt. Sie bietet eine den bisherigen Bänden entsprechende Lesetypographie inklusive aller Paratexte. Auch zur ,bloßen“ Erstellung eines gedruckten Buches wären allerdings die Werkzeuge der digitalen Domäne hilfreich (z.B. ein gemeinsames Register zur Disambiguierung von Personennamen). ${ }^{5}$ Die Werkzeuge, die das Team am ACDH-ÖAW bzW. am INZ dafür entwickelt haben, sind mit überschaubarem Zusatzaufwand so auszugestalten, dass neben dem unmittelbaren Gewinn für die Edition auch Nachnutzung und Datenoffenlegung im Sinne von Open Access und Open Data für die Allgemeinheit möglich ist.

Die digitale Edition ermöglicht in vielerlei Hinsicht Mehrwert gegenüber dem Paradigma Buch: ${ }^{6}$

- bandübergreifende facettierte Volltextsuche,

- gegenüber dem Printprodukt erweiterte Darstellungs- und Filterungsoptionen,

- Ergänzung durch Faksimiles insbesondere im erwähnten Bereich der „Brandakten“,

- Erweiterbarkeit im Falle neuer Quellenfunde auch unabhängig vom weiterhin erscheinenden Printprodukt,

- die Ergänzung durch Normdaten und Linked Data,

- die Erschließung neuer Publikumskreise durch erweiterte Sichtbarkeit,

- das Zurverfügungstellen und Archivieren der der Edition zugrunde liegenden Daten.

\section{Konkrete Umsetzung - Editionsworkflow}

Bei der seit 2017 geplanten und mit Anfang 2018 in Umsetzung befindlichen Umstellung des editorischen Vorgehens werden Schlüsselstellen auf dem Weg zu einer digitalen Edition virulent. Besondere Aufmerksamkeit gilt hierbei zu richten auf:

- den gemeinsam mit dem ACDH-ÖAW entwickelten Workflow (siehe Abbildung $1^{7}$ )

» Eingabe in MS Word (.docx und textsortenspezifische Formatvorlagen),

》 Auxiliardaten zur Erstellung der Indizes, modelliert in einer APIS-Instanz, ${ }^{8}$ d.i. einer relationalen Datenbank und dem dazugehörigen Frontend, ${ }^{9}$

»XSL-Transformation aus .docx in TEl.xml.

5 Dazu vgl. SAHLE, Editionsformen, Teil 2, 61f.

6 Zur Navigierbarkeit des Buchs vor dem Hintergrund des Hypertexts vgl. unter vielen anderen: Patrick SAHLE, Digitale Editionsformen. Zum Umgang mit der Überlieferung unter den Bedingungen des Medienwandels. Teil 3: Textbegriffe und Recodierung. [Finale Print-Fassung], Norderstedt 2013 (Schriften des Instituts für Dokumentologie und Editorik Bd. 9); aus literaturwissenschaftlicher Sicht Thomas Eder / Samo Kobenter / Peter Plener, Hg., Seitenweise. Was das Buch ist, Wien 2010; aber auch schon Tim BuLKELEY, Form, Medium and Function: The Rhetorics and Poetics of Text and Hypertext in Humanities Publishing, in: International Journal of the Book 1 (2003), 317-327.

7 Die graphviz-Quelldatei des Diagramms ist verfügbar online unter: https://gist.github.com/skurzinz/c617fe 4 b75d577cc4ea5972bbfeeoed2 (17.06.2019).

8 Siehe https://mpr.acdh.oeaw.ac.at/ (17.06.2019).

9 Datenbank und Frontend entstammen einem Kooperationsprojekt zwischen drei ÖAW-Instituten, namentlich dem Austrian Centre for Digital Humanities ACDH-ÖAW, dem Institut für Stadt- und Regionalforschung ISR, sowie dem Institut für Neuzeit- und Zeitgeschichtsforschung INZ; Details siehe online unter: https://www.oeaw.ac.at/acdh/projects/apis/ (17.06.2019). 
- die hybride Erscheinungsweise („single source publishing”)

»Erzeugung der PDF-Druckvorlage aus den XML-Daten (via pdf(lua)LaTeX),

» digitale Edition mithilfe einer XML-Datenbank und Präsentationslayer (strikte Trennung von Daten und Applikation).

Für Herausforderungen sorgt die Modellierung und Erstellung von notwendigen Auxiliardaten, wie etwa Named Entities mit diachroner Varianz (Beispiel: Namensänderung durch Personenstands- oder Standesänderungen; Umgang mit Mehrsprachigkeit bei Ortsnamen) sowie die Komplexität von Sachregister-Vokabularien und -Thesauri.

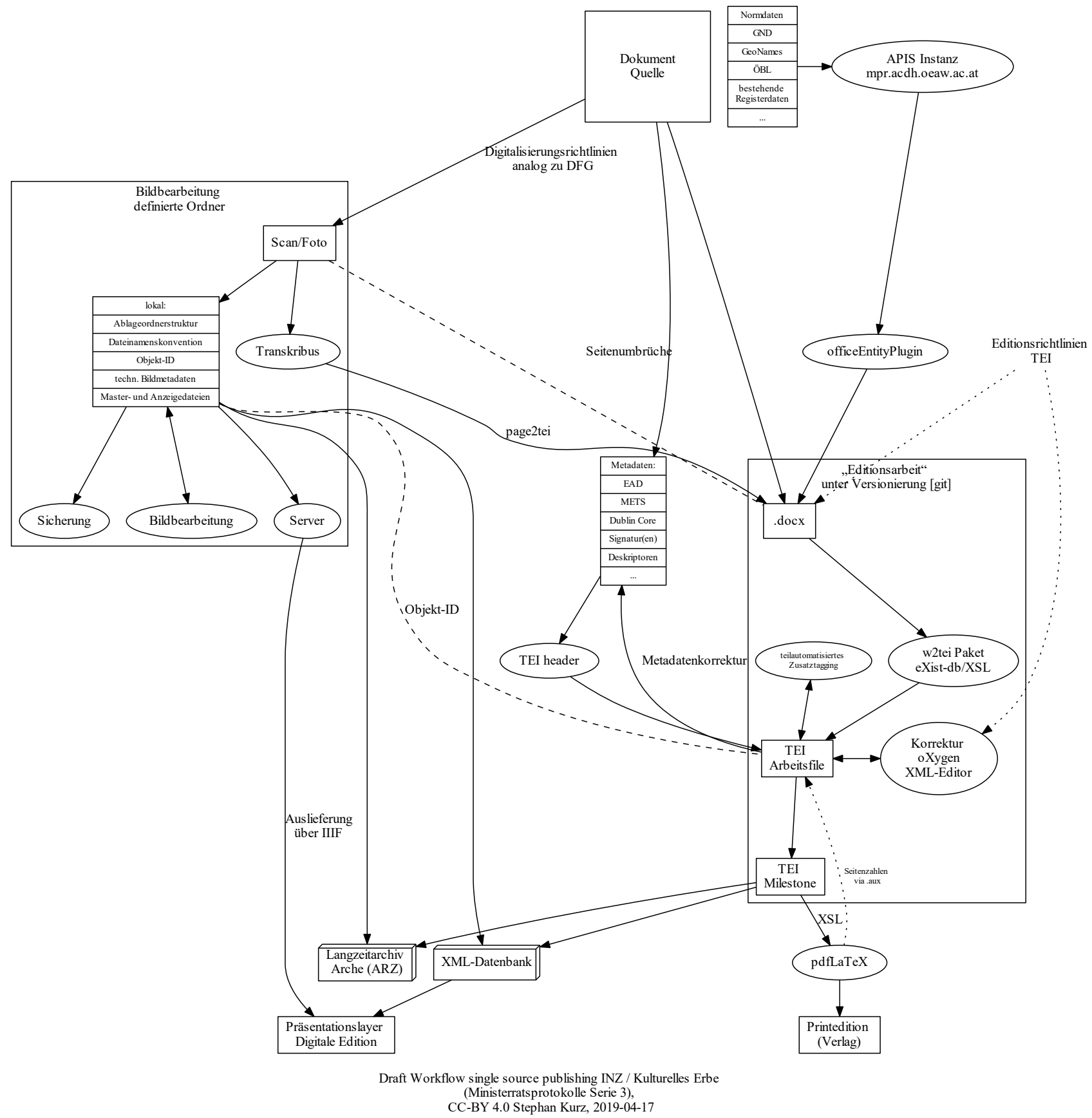

Abb. 1: Editionsworkflow 


\subsection{Textkonstitution}

Für die bisher erschienenen Bände wurde bei der Erstellung der Protokolltexte nichtstrukturierter Text verwendet, wenngleich die textsortenspezifische Struktur der Protokolle sich stets wiederholt - diese ist mittlerweile in Form von Formatvorlagen für MS Word festgehalten, was die Arbeit mit den Texten erleichtert: Auf einen Protokolltitel mit Datums- und Sitzungsbezeichnung folgt ein Kopfregest, das im Wesentlichen den Inhalt der separat archivierten Tagesordnungsbücher wiedergibt (An- und Abwesenheiten der Mitglieder, Bezeichnung der in der Sitzung behandelten Tagesordnungspunkte, datierte Bestätigungen der Einsichtnahme durch die Mitglieder). Mit einem textkritischen Apparat sowie einem Stellenkommentar zu Bezügen wird im Anschluss daran der Wortlaut des überlieferten Protokolls gegeben.

Die Datenmodellierung der Auxiliardaten folgt dem Datenmodell von APIS; dabei kommen kontrollierte Vokabularien zur Anwendung, um die Beziehungen zwischen den Grundentitäten (Personen, Institutionen, Orte, Werke, Ereignisse) abzubilden.

Die solcherart erzeugten Netzwerke von Entitäten werden für den Zweck der XML-Edition sowie der daraus zu generierenden Druckvorlage in einzelne Registerdateien exportiert (dies geschieht nach den Vorgaben der TEl-Richtlinien, also als listPerson, listPlace, listorg etc.). Sie werden darüber hinaus aber auch als Linked Open Data zur Nachnutzung zur Verfügung gestellt: Das APIS-Webinterface bietet sowohl Exportmöglichkeiten als auch eine API an.

Die Auxiliardaten werden mithilfe des erwähnten Plugins für MS Word über die API aus der Datenbankinstanz http://mpr.acdh.oeaw.ac.at/ bereits in diesem Schritt von den Editoren eingepflegt und kontrolliert.

Bei der Erarbeitung der Textgrundlagen für die Edition der Ministerratsprotokolle 1893-1900 im Rahmen des FWF-Projekts von Anatol Schmied-Kowarzik und Richard Lein hat das Editionsteam in Ergänzung zu den etablierten Transkriptionsmethoden zuletzt erfolgreich die Transkription der maschinschriftlich vorliegenden Archivquellen mithilfe der Software Transkribus ${ }^{10}$ erprobt; durch den Umfang der in solcher Form vorliegenden Quelldokumente (ca. $90 \%$ des Projektumfangs) ist zu erwarten, dass dadurch substanzielle Erleichterung der Arbeitsweise erreichbar ist.

\subsection{Transformation in TEI-XML}

Der konstituierte Text wird anschließend aus den einzelnen Word-Dokumenten mithilfe der Transformationsszenarien der eXist-Applikation w2tei in mehreren Schritten in den Richtlinien des TEI-Konsortiums entsprechende XML-Dokumente übersetzt.

\subsection{Drei Endpunkte der hybriden Edition}

Die auf diese Weise erzeugten XML-Dokumente sind die Grundlage erstens für die digitale Edition, für die wir aktuell - aufbauend auf generischen Transformationen von TEI nach HTML - einen Präsentationslayer entwickeln. Zweitens werden daraus auch die LaTeX-Daten generiert, die den PDF-Druckvorlagen zugrunde liegen. Die Editionsleistung besteht also darin, valide XML-Dokumente zu erstellen, die dann ihrerseits die Basis für die ,Benutzerschnittstellen' (d.i. Buch und Onlinefassung) bilden. Sie

10 Vgl. https://read.transkribus.eu/ (17.06.2019). 
werden drittens im Langzeitarchiv ARCHE abgelegt und mit Metadaten versehen, um ihre langfristige Verfügbarkeit und Benutzbarkeit sicherzustellen.

\section{Stand der Arbeiten}

Die Umstellung auf eine hybride (digitale und gedruckte) Erscheinungsweise hat seit der Präsentation im Rahmen der Digital Humanities Austria 2018-Konferenz in Salzburg folgende Fortschritte zu verzeichnen:

- Einrichtung und teilweise Befüllung einer relationalen Datenbank zur Erfassung der Personen, Institutionen und Orte als Backend für die semantische Erschließung/Verlinkung (online) sowie für die Registererstellung (print), basierend auf dem Datenmodell von APIS - https://mpr.acdh. oeaw.ac.at/

- Programmierung eines Plugins für MS Word zur Verbindung der o.g. Datenbank mit den Protokolltexten - https://github.com/dariok/officeEntityPlugin/

- Workflowentwicklung .docx > TEl inklusive der Einbindung der o.g. Auxiliarressourcen - siehe Abb. 1

- Die genannten Schritte konnten in mehreren Publikationen und Vorträgen einem weiteren Publikum insbesondere im disziplinären Umfeld der Digital Humanities gegenüber dokumentiert werden. ${ }^{11}$

Zusätzlich zur ursprünglichen Beschränkung auf die neu zu edierenden Ministerratsprotokolle Cisleithaniens ab 1867 konnten in Absprache mit dem Verlag der ÖAW auch die ca. 2500 Retrodigitalisate der Protokolle 1848-1867 in das TEI-Format und unter einer freien Lizenz überführt werden. Über 40 Jahre historischer Grundlagenarbeit zu 20 Jahren Regierungstätigkeit der Habsburgermonarchie werden damit einer weltweiten Öffentlichkeit zugänglich.

Das mittelfristige Ziel ist eine gemeinsame Applikation für die beiden Serien der Edition, also für Retrodigitalisate (1848-1867) und born digital Digitale Edition (1867-1918). Deren Entwicklung ist aktuell noch nicht abgeschlossen, sie profitiert allerdings wesentlich von den Vorarbeiten und dem Austausch über das Kompetenznetzwerk Digitale Edition (KONDE-AT). ${ }^{12}$

\section{Zusammenfassung}

Die Umstellung auf ein hybrides Editionsverfahren ist eine große Herausforderung. Alle Variablen und alle Abläufe müssen abgewogen werden, um dem Ziel einer zeitgemäßen Repräsentation historischer Quelldokumente sowohl im Druck als auch in der Onlinefassung gerecht zu werden.

11 Vgl. Stephan KuRz / Ksenia ZAYTSEVA, Herausforderungen für Thementhesauri und Sachregister-Vokabularien zur Erschließung im Kontext des digitalen Editionsprojekts Cisleithanische Ministerratsprotokolle, in: Patrick Sahle, Hg., DHd 2019 Digital Humanities: multimedial \& multimodal. Konferenzabstracts, Frankfurt am Main / Mainz 2019; Wladimir FISCHER-NEBMAIER u. a., Der Regierung in die Karten geschaut. Hybride Edition der österreichischen/cisleithanischen Ministerratsprotokolle (1867-1918), in: ÖAW, Hg., Auf lange Sicht zu neuen Perspektiven. Langzeitvorhaben der Österreichischen Akademie der Wissenschaften, Wien 2019 (im Druck); Wladimir FISCHER-NEBMAIER, Die Protokolle des cisleithanischen Ministerrates und die Julikrise 1914, Die Julikrise 1914 - Revisited (10. Workshop Internationale Geschichte) [Vortrag], Wien 2018.

12 Vgl. http://www.digitale-edition.at/ (17.06.2019); nachnutzbarer Code online unter: https://github.com/KONDE-AT (17.06.2019). 
Die Anforderungen einer besonderen Textsorte, für die im Bereich der digitalen Editorik noch kaum Vorbilder zur Verfügung stehen, lassen uns dabei Neuland betreten. ${ }^{13}$ Es geht um die erweiterte Erschließung und digitale Archivierung des kulturellen Erbes der administrativen Organe der Habsburgermonarchie und Österreich-Ungarns.

Mit der digitalen / hybriden Edition der Ministerratsprotokolle erschließen wir eine zentrale Quelle für die künftige historische Forschung, die einem größeren Kreis von Interessierten offenstehen wird - in einer zukunftssicheren Form und neu erschlossen für die weiteren Jahre.

\section{Literaturverzeichnis}

Tim BulKeley, Form, Medium and Function: The Rhetorics and Poetics of Text and Hypertext in Humanities Publishing, in: International Journal of the Book 1 (2003), 317-327, online unter: https://web.archive.org/ web/20161123071115/http://bigbible.org/papers/bookconf.htm (17.06.2019).

Thomas Eder / Samo Kobenter / Peter Plener, Hg., Seitenweise. Was das Buch ist, Wien 2010.

Wladimir FISCHER-NeBMAIER, Die Protokolle des cisleithanischen Ministerrates und die Julikrise 1914, Die Julikrise 1914 - Revisited (10. Workshop Internationale Geschichte) [Vortrag], Wien 2018.

Wladimir FISCHER-NeBMAIER u. a., Der Regierung in die Karten geschaut. Hybride Edition der österreichischen/ cisleithanischen Ministerratsprotokolle (1867-1918), in: ÖAW, Hg., Auf lange Sicht zu neuen Perspektiven. Langzeitvorhaben der Österreichischen Akademie der Wissenschaften, Wien 2019 (im Druck).

Stephan KURZ / Ksenia ZAYTSEVA, Herausforderungen für Thementhesauri und Sachregister-Vokabularien zur Erschließung im Kontext des digitalen Editionsprojekts Cisleithanische Ministerratsprotokolle, in: Patrick Sahle, Hg., DHd 2019 Digital Humanities: multimedial \& multimodal. Konferenzabstracts, Frankfurt am Main / Mainz 2019, 304f., online unter: https://zenodo.org/record/2596095 (17.06.2019).

Helmut RumPLeR, Hg., Die Ministerratsprotokolle Österreichs und der Österreichisch-Ungarischen Monarchie 1848-1918, Ser. 1: Die Protokolle des Österreichischen Ministerrates 1848-1867, Einl.-Bd.: Ministerrat und Ministerratsprotokolle 1848-1867. Behördengeschichtliche und aktenkundliche Analyse, Wien 1970.

Patrick SAHLE, Digitale Editionsformen. Zum Umgang mit der Überlieferung unter den Bedingungen des Medienwandels. Teil 1: Das typografische Erbe. [Finale Print-Fassung], Norderstedt 2013 (Schriften des Instituts für Dokumentologie und Editorik Bd. 7), online unter: https://kups.ub.uni-koeln.de/5351/ (17.06.2019).

Patrick SAHLE, Digitale Editionsformen. Zum Umgang mit der Überlieferung unter den Bedingungen des Medienwandels, Teil 2: Befunde, Theorie und Methodik [Finale Print-Fassung], Norderstedt 2013 (Schriften des Instituts für Dokumentologie und Editorik Bd. 8), online unter: https://kups.ub.uni-koeln.de/5352/ (17.06.2019).

Patrick SAHLE, Digitale Editionsformen. Zum Umgang mit der Überlieferung unter den Bedingungen des Medienwandels. Teil 3: Textbegriffe und Recodierung. [Finale Print-Fassung], Norderstedt 2013 (Schriften des Instituts für Dokumentologie und Editorik Bd. 9), online unter: https://kups.ub.uni-koeln.de/5353/ (17.06.2019).

Patrick SAHLE, Zwischen Mediengebundenheit und Transmedialisierung. Anmerkungen zum Verhältnis von Edition und Medien, in: editio 24 (2010), 23-36.

Johannes STIGLER, Anmerkungen zu einem generischen Verständnis des Begriffes „Digitale Edition“, in: Anne Bohnenkamp-Renken / Elke Richter, Hg., Brief-Edition im digitalen Zeitalter, Berlin 2013 (Beihefte zu editio 34), 43-52.

13 Das Fehlen von Vorbildern zeigt eine Suche nach ähnlich gelagerten Quellen in den gängigen Editionskatalogen (Dig-EdCat online unter: https://dig-ed-cat.acdh.oeaw.ac.at (17.06.2019) und IDE-Katalog von Patrick Sahle online unter: http:// digitale-edition.de/ (17.06.2019)). 


\title{
Community involvement for transcribing historical correspondences of South Tyrolean interest
}

\author{
A DI-ÖSS 1 USE CASE
}

Alexander König [1], Verena Lyding [1], Elisa Gorgaini [1], Georg Grote [2] \& Monica Pretti [1]

[1] Eurac Research, Institute for Applied Linguistics

Alexander.Koenig@eurac.edu, https://orcid.org/0000-0002-8540-2396

Verena.Lyding@eurac.edu, https://orcid.org/0000-0002-2301-6860

elisa.gorgaini@gmail.com, https://orcid.org/0000-0003-3292-8914

Monica.Pretti@eurac.edu, https://orcid.org/0000-0002-3999-6103

[2] Eurac Research, Institute for Minority Rights

Georg.Grote@eurac.edu, https://orcid.org/0000-0002-3234-5623

Keywords: Crowdsourcing, Transcription, History, Linguistics, OCR, Citizen Science

\begin{abstract}
We present a local research and citizen science initiative for the crowdsourcing-based enrichment and analysis of authentic handwritten postcards and letters, sent by South Tyrolean front-line soldiers to their families at home during the World Wars.
\end{abstract}

In this article, we present a citizen science initiative for the documentation and transcription of historical handwritten postcards and letters by means of crowdsourcing. The documents are (mostly) authentic communications between South Tyrolean soldiers and their families at home from the First World War - during which the region of South Tyrol was right on the front lines - and the Second World War.

The presented initiative, carried out in cooperation between the Institute for Applied Linguistics (IAL) and the Institute for Minority Rights (IMR) ${ }^{3}$ at Eurac Research, is one of a number of use cases that are part of the infrastructure project DI-ÖSS ${ }^{4}$. The DI-ÖSS project is piloting a digital infrastructure for language data within the region of South Tyrol, which aims at exploring and exploiting synergies between actors in the language domain. This infrastructure is envisioned growing in a bottom-up manner where the special situation of the various institutions in South Tyrol that are dealing with language data is taken into account. In the currently running pilot phase, these synergistic possibilities are showcased through

\footnotetext{
$1 \quad$ Digitale Infrastruktur für das Ökosystem Südtiroler Sprachdaten und -dienste (Digital infrastructure for the ecosystem of South Tyrolean language data and services).

2 http://www.eurac.edu/en/research/autonomies/commul/Pages/default.aspx (23.04.2019).

3 http://www.eurac.edu/en/research/autonomies/minrig/Pages/default.aspx (23.04.2019).

4 http://www.eurac.edu/en/research/projects/Pages/projectdetail4262.aspx (23.04.2019).
} 
various specialized use cases which are implemented among the partners in the DI-ÖSS project. These have been selected to represent as wide a range as possible of different types of institutions relevant to the local language situation. The partners are Eurac Research (IAL and IMR), the library "Landesbibliothek Dr. Friedrich Teßmann", the language unit of the institute for culture - "Sprachstelle" ${ }^{\prime \prime}$, and the online news and community portal salto.bz ${ }^{7}$.

This use case is related to the "letter project" which aims at collecting as many "local" correspondences as possible from the $20^{\text {th }}$ century in South Tyrol, which was a tumultuous time, especially with the two World Wars and the annexation to Italy affecting the region dramatically. The collection of documents that are the basis for the project has been compiled over the past years at the IMR from local citizens all over South Tyrol. They have been actively sought out through open calls to local magazines ${ }^{8}$, journal contributions $^{9}$ and radio broadcasts about the project. This approach is meant to put the man on the street in the focus of the historical research, investigating the lifestyle and emotions of the people by analyzing the content of the letter exchanges. So far about 13,000 items have been collected. For obvious reasons, there is a peak of material during the two World Wars because that was the time when couples and families were forced apart by circumstances which created the need to communicate via mail. This special situation also makes the collected data particularly interesting for linguistic research as it is the rare case of getting a significant amount of texts from "low literacy writers", i.e. people that probably wrote very little since they left school and suddenly found themselves in a situation where they needed to communicate via written texts. Apart from these interesting features, the special language situation in South Tyrol also means that there are many instances of code-switching to be expected within the texts, be it between standard German and the various local dialects, or - especially after the annexation - also between German and Italian. Finally, from a perspective of diachronic language change it will be relevant to compare all of these features across the course of the century.

At the current state of the project, the collaborating historian at the IMR has created a network of local families as data providers. He has been collecting several thousand missives as original documents and scanned them to JPEG-images. To enable research on characteristics of the correspondences from a linguistic as well as a historical perspective, the data needs to be made accessible not only in digital but also in machine-readable form. In particular in order to analyze the texts in a systematic way, they need to be properly equipped with metadata and transcribed. This is the part of the project that employs a citizen science approach to complete the laborious task of transcribing handwritten texts and encoding key details of the correspondences into formalized metadata. Metadata will include information about the circumstances of the correspondence, e.g. the date and place of sending, the name of the sender and the recipient, as well as their relationship (e.g. mother/son or husband/wife), and information on the quality of the writing or the script being used.

Regarding the transcription of handwritten texts with different scripts, the human contribution is of particular relevance, as software cannot easily support this. The strategy implemented in this project is to set up a crowdsourcing site to engage the local population in this project. Already during the collection of the letters, it became obvious that the people in South Tyrol are very much interested in this insight

http://www.tessmann.it/en/home.html (23.04.2019).

https://www.kulturinstitut.org/sprachstelle.html (23.04.2019).

https://www.salto.bz (23.04.2019).

Georg GROTE, Ein kollektives Gedächtnis für Südtirol, in: ACADEMIA 78 (2018), 41.

9 Mechthild PÖRnBACHER u.a., "Mir geht es gut ...": Feldpostkarten Südtiroler Soldaten im Ersten Weltkrieg, in: Der Schlern. Monatszeitschrift für Südtiroler Landeskunde 88/07-08 (2014), 4-21. 
into the past of their region (and often also their families). Therefore, in this use case we can join the need for help from the general populace with the great interest of people in the region in interacting with their (family) history. This becomes central especially in this year, as it marks the centennial of the annexation of South Tyrol to Italy in 1919. The crowdsourcing project will be set in a context of multiple interrelated events to commemorate and highlight this historical period via engaging directly with the population and the families of those who lived through this time.

The citizen science involvement is organized in two steps: step (1) serves to systematically label the correspondences with metadata, step (2) caters for the transcription of the handwritten texts. Both steps will be completed by crowdworkers, who should be local citizens, through editing interfaces that run in standard web browsers.

For the metadata collection a local installation of the crowdsourcing framework PYBOSSA ${ }^{10}$ has been installed at Eurac Research. PYBOSSA was chosen, because it has been released as open source ${ }^{11}$ which meant that it could be installed by the project partners within their own IT infrastructure without having to rely on an external service provider. For the same reason it can also easily be adapted to the specific needs of this project. The interface displays the scanned document as an image and provides a number of descriptive fields related to the document (i.e. metadata such as name of the sender and the recipient, format of the correspondence, script, date, etc.), which should be completed by the crowdworkers. PYBOSSA can be configured to send the same task (i.e. providing metadata to a specific image) to multiple users and thus providing greater reliability of the results. And as the software is installed on local Eurac research infrastructure, the extraction of the results can easily be automated, and they can also automatically be converted into a suitable metadata format for further use.

The transcription of the text itself will be done in a second independent crowdsourcing phase, once the metadata has been collected. For this, several tools are currently under evaluation to find the one best suited to the needs of the project. Most likely the tool Transkribus ${ }^{12}$ will be employed for this task. It has been developed in the context of the READ project ${ }^{13}$ to specifically cater to the needs of archives in dealing with old handwritten documents. Transkribus is offered as a feature-rich Java application where the user can upload their documents and use built-in automatic handwriting recognition algorithms to generate a transcription, which can then further be worked on manually. While the results are often not very good out of the box, they can be used as a basis for manual transcription to provide a starting point for the crowdworkers. At the same time, those manual transcriptions can be fed back into the algorithms and improve them over time, meaning the automatic recognition will get better and better, especially in a project as described in this article where there exist a lot of texts from the same author with the same handwriting. As we want people to participate over the internet, we plan to make use of the Transkribus web interface ${ }^{14}$, which does not provide all the functionality of the full Transkribus desktop application, but has all the features which will be needed for the transcription and has the additional advantage of providing a more streamlined interface which will work better for people more inexperienced with the use of complex transcription applications.

10 https://pybossa.com (23.04.2019).

11 https://github.com/Scifabric/pybossa (23.04.2019).

12 Philip KAHLE u. a., Transkribus - A service platform for transcription, recognition and retrieval of historical documents, 14th IAPR International Conference on Document Analysis and Recognition (ICDAR), Kyoto 2017, 19-24, DOI: 10.1109/ICDAR.2017.307.

13 https://read.transkribus.eu/ (23.04.2019).

14 https://transkribus.eu/r/read/projects/ (23.04.2019). 
It has been shown in various other projects throughout Europe, for example the Dutch "Gekaapte Brieven"15 or the large-scale European project "Europeana 1914-18"16, that the general public is easily engaged in this kind of documentation and transcription tasks. There is quite some interest in historical letters and people enjoy interacting with the research community. On the other hand, the researchers are in need of support from the community to carry out the task of transcription. In this case, it will help to explicitly involve the local population, both because they are likely to have a stronger interest in the local history and also because they might have special knowledge (geographical or historical details) and will be familiar with the dialect variants of South Tyrolean German which can help with the transcription process. In order to correctly transcribe older German scripts (e.g. Kurrent), it might also be useful to specifically target an older age group that might have some experience with this kind of script. In the perspective of involving generations from the pre-digital era, Europeana has also shown that crowdsourcing does not have to stay confined to the internet but can be organized through so-called Transcribathons ${ }^{17}$. These are community involvement events where researchers first introduce a project through a presentation and afterwards the public can help the research process by transcribing and annotating the materials. Within the DI-ÖSS project, these kinds of events are planned in order to spark the public's interest in the research and to encourage members of the local community to participate as equals.

\section{Literature}

Georg Grote, Ein kollektives Gedächtnis für Südtirol, in: ACADEMIA 78 (2018), 41.

Philip KAHLE U. a., Transkribus - A service platform for transcription, recognition and retrieval of historical documents, 14th IAPR International Conference on Document Analysis and Recognition (ICDAR), Kyoto 2017, 19-24, DOI: 10.1109/ICDAR.2017.307.

Mechthild PÖRNBACHER u.a., "Mir geht es gut ...": Feldpostkarten Südtiroler Soldaten im Ersten Weltkrieg, in: Der Schlern. Monatszeitschrift für Südtiroler Landeskunde 88/07-08 (2014), 4-21.

http://gekaaptebrieven.nl/ (23.04.2019).

https://github.com/Scifabric/pybossa (23.04.2019).

https://pro.europeana.eu/project/europeana1914-1918 (23.04.2019).

https://pybossa.com (23.04.2019).

https://transcribathon.com/ (23.04.2019).

https://transkribus.eu/r/read/projects/ (23.04.2019).

http://www.eurac.edu/en/research/autonomies/commul/Pages/default.aspx (23.04.2019).

http://www.eurac.edu/en/research/autonomies/minrig/Pages/default.aspx (23.04.2019).

http://www.eurac.edu/en/research/projects/Pages/projectdetail4262.aspx (23.04.2019).

https://www.kulturinstitut.org/sprachstelle.html (23.04.2019).

https://www.salto.bz (23.04.2019).

http://www.tessmann.it/en/home.html (23.04.2019).

15 http://gekaaptebrieven.nl/ (23.04.2019).

$16 \mathrm{https} / / /$ pro.europeana.eu/project/europeana1914-1918 (23.04.2019).

17 https://transcribathon.com/ (23.04.2019). 


\title{
Soziale Netzwerkanalyse und Erzählschemata
}

\author{
Eine explorative Vorstudie
}

\section{Friedrich Michael Dimpel}

FAU Erlangen und TU Darmstadt

mail@dimpel.de

Keywords: Literary Network Analysis, Computational Literary Studies, Literary Annotation

\begin{abstract}
This study deals with nine texts which belong to three different narrative schemes: ,Brautwerbungsschema', ,Freier-Befreiungs-Geschichten' und ,Mahrtenehe-Erzählungen'. Each category is represented by three texts. While Literary Network Analysis can clearly distinguish texts of the ,Brautwerbungsschema' type from the other two types, there are no clear differences between ,Freier-Befreiungs-Geschichten' and ,Mahrtenehe-Erzählungen'.
\end{abstract}

\section{Literarische Netzwerkanalyse}

Die Literarische Netzwerkanalyse hatte keinen guten Start: Franco Moretti hat mit einfachen Netzwerkanalysen, die nicht gewichtet und nicht gerichtet waren, ${ }^{1}$ Shakespeare-Dramen analysiert. Von dem überraschenden Befund, dass sich die Bedeutung der Hauptfiguren nicht in den Netzwerkgraphen visualisieren lässt, hat sich Moretti dazu verleiten lassen, vorzuschlagen, konventionelle literaturwissenschaftliche Kategorien wie Hauptfigur versus Nebenfiguren oder ,runde“ versus ,flache‘ Figur zu überdenken: „In Anbetracht der Verteilung spricht nichts für diese Dichotomien, sie macht eher eine radikale Neukonzeption der Figuren und ihrer Hierarchie erforderlich." 2

Deutlich differenzierter operiert die Pionierstudie zur Netzwerkanalyse in der deutschen Literaturwissenschaft von Peer Trilcke: Trilckes gewichtete Netzwerkgraphen ermöglichen durchaus eine Unterscheidung von eher zentralen und eher peripheren Figuren. ${ }^{3}$ Auf der Grundlage von solchen Plots, die auf einer genaueren Datengrundlage beruhen, besteht keine Notwendigkeit, gängige Unterscheidungen von Hauptfiguren und Nebenfiguren ${ }^{4}$ zu verabschieden. ${ }^{5}$ Sicherlich darf es weniger darum ge-

1 Durchaus sehenden Auges, Moretti kommentiert diesen Umstand mit einem „Das kann nicht richtig sein.“ Vgl. Franco MORETTI, Distant Reading. Aus dem Englischen übersetzt von Christine Pries, Konstanz 2016 (2013), 194.

2 Ebd., 198f.

3 Peer TRILCKE, Social Network Analysis (SNA) als Methode einer textempirischen Literaturwissenschaft, in: Philip Ajouri /o Katja Mellmann / Christoph Rauen, Hg., Empirie in der Literaturwissenschaft, Münster 2013 (Poetogenesis.Studien zur empirischen Anthropologie der Literatur 8), 201-247, hier 227-236.

4 Vgl. etwa Fotis JANNIDIS, Figur und Person. Beitrag zu einer historischen Narratologie, Berlin / New York 2004 (Narratologia 3), 104, der als Unterscheidungskriterium die Partizipation der Figur an Ereignissen in der erzählten Welt vorschlägt.

5 Zuletzt wurden SNA-Methoden verwendet, etwa bei Michael VAUTH, Netzwerkanalyse narrativer Informationsvermittlung in Dramen, in: Patrick Sahle, Hg., DHd 2019. Digital Humanities: multimedial \& multimodal. Konferenzabstracts, Köln 2019, 184-188, der Dramen von Heinrich von Kleist, insbesondere die „Familie Schroffenstein“ untersucht. Benjamin KRAUTTER / 
hen, überraschende Ergebnisse als ,distant reading mit einem „grellen Marktgeschrei der Innovation“6 zu vermarkten, sondern eher darum, um akkurate Daten zu kämpfen - auch wenn das mit Blick auf die Problemlage in narrativen Texten oft kein einfaches Geschäft darstellt, so dass man sich manchmal durchaus auch mit Annäherungen helfen muss.

Bei Trilcke dient die gleichzeitige Bühnenpräsenz im Drama als Indikator für soziale Interaktion und damit als Grundlage für die Netzwerkanalyse. Nun lässt sich diskutieren, ob eine akkuratere Erfassung von Figuren-Interaktionen als die gemeinsame Bühnenpräsenz im Drama möglich ist. Zumindest noch derzeit hat eine genauere Erfassung eine manuelle Annotation zur Voraussetzung, die idealerweise noch durch ein Inter-Annotator-Agreement abzusichern wäre: Wenn man etwa annotiert, welche gesprochene oder gedachte Figurenrede oder welcher Erzählerbericht von Figurenhandlungen einen Bezug auf welche andere Figuren enthält, lässt sich eine Beziehung zwischen zwei Figuren eher in einer Weise modellieren, wie sie bei sozialwissenschaftlichen Netzwerkanalysen üblich ist. ${ }^{7}$

Solche Studien erfordern aber derzeit noch einen erheblichen Annotationsaufwand, so dass bei längeren mittelhochdeutschen Erzählungen alternative Parameter gefunden werden müssen, die eine Modellierung der Figureninteraktion erlauben, zumal hier nicht auf Regieanweisungen wie im Drama der Moderne zurückgegriffen werden kann. Im Rahmen der vorliegenden Studie wird mit Figurenreferenzen operiert sowie zum einen untersucht, wie weit zwei Figurenreferenzen voneinander entfernt im Text stehen, und zum anderen, welche Figurenreferenzen in der Figurenrede welcher Figur vorkommen.

Eine Option, die Figurenreferenzen zu ermitteln, wäre die Nutzung des Stuttgarter Tools CRETAnno, das im Rahmen des Stuttgarter DH-Zentrums CRETA von Nora Ketschik und André Blessing entwickelt wurde. Mit CRETAnno ist eine teilautomatische Erkennung von Figurenreferenzen möglich. ${ }^{8}$ CRETAnno findet Figurenreferenzen in diesen Texten, also Ausdrücke, die eine Figur benennen oder auf eine Figur verweisen. Dabei werden auch Referenzen wie diu „frouwe“ einer Figur zugeordnet, Pronomina werden allerdings nicht erfasst. Zudem besteht derzeit noch ein erheblicher Aufwand mit der manuellen Korrektur der erfassten Referenzen. Wann es mit Hilfe von regelbasierten oder maschinellen Verfahren möglich wird, automatisch zu disambiguieren, ob sich eine Referenz wie „herzogin“ im „Parzival“ auf Orgeluse oder Jeschute bezieht, ist momentan noch nicht absehbar. Da jedoch Figurenrede in mittelhochdeutschen Texten derzeit ohnehin noch manuell annotiert werden muss, werden hier sowohl die Figurenreferenzen als auch die Textsegmente, die Figurenrede enthalten, in einem manuellen Annotationsverfahren erfasst, bei dem auch pronominale Referenzen berücksichtigt werden. ${ }^{9}$

Janis PAGEL, Klassifikation von Titelfiguren in deutschsprachigen Dramen und Evaluation am Beispiel von Lessings ,Emilia Galotti', in: Patrick Sahle (Hg.), DHd 2019. Digital Humanities: multimedial \& multimodal. Konferenzabstracts, Köln 2019, 160-164, zeigen, dass die Betweenness Centrality ein geeigneter Parameter ist, um Hauptfiguren von Nebenfiguren zu unterscheiden (Closeness dagegen weniger; so im mündlichen Vortrag). Thomas PROISL u. a., Dependenzbasierte syntaktische Komplexitätsmaße, in: Patrick Sahle, Hg., DHd 2019. Digital Humanities: multimedial \& multimodal. Konferenzabstracts, Köln 2019, 270-273, verwenden u.a. Zentralitätsmaße zur Ermittlung von Komplexität in Romanen.

6 TRILCKE, Social Network Analysis, 247.

7 Vgl. Friedrich Michael DIMPEL, Novellenschätze narratologisch auszeichnen und analysieren am Beispiel Victor von Scheffels ,Hugideo' und der sozialen Netzwerkanalyse, in: LiLi 47 (2017), 87-108, DOI:10.1007/s41244-017-0044-8, $104-108$.

8 Vgl. https://www.creta.uni-stuttgart.de/tools/ (26.06.2019).

9 Mein Dank für die Annotationsarbeit gilt den studentischen Hilfskräften, die an diesem Projekt beteiligt waren: Sarah Dönges, Barbara Endl, Stefanie Gatzka und Sjard Salzmann. 


\section{Erzählschemata}

Mit einem ähnlichen Verfahren, das ebenfalls auf den Distanzen von Figuren-Referenzen im Text beruht, die mit den Verfahren der Netzwerkanalyse ausgewertet werden, haben Hettinger / Becker / Reger / Jannidis / Hotho untersucht, ob es möglich ist, eine Differenzierung von Roman-Subgenres quantitativ nachzuvollziehen - allerdings mit einem negativen Befund. ${ }^{10}$ Ich vermute, dass die Ergebnisse von netzwerkanalytischen Verfahren in hohem Maße abhängig von der linearen Entwicklung der Figurenkonstellation in Texten sein dürften. Zu bedenken ist, dass mit der gleichen Figurenkonstellation ein Drama, einen Roman oder eine Kurzerzählung gestaltet werden kann. Eventuell könnte dies auch für verschiedene Romansubgenres gelten, obwohl hier noch in stärkerem Maße als bei einer Differenzierung etwa von Drama, Roman und Kurzerzählung jeweils eher noch Figurentypen mit subgenrespezifischen Eigenheiten zu erwarten sind.

Mein Vorhaben setzt daher auf einer anderen Ebene an: Untersuchen will ich, ob sich Textabschnitte, die einem bestimmten Erzählschema ${ }^{11}$ oder Erzählkern ${ }^{12}$ folgen, mittels Netzwerkanalyse gruppieren lassen. Meine Thesen sind: (1) Befunde einer Literarischen Netzwerkanalyse könnten mit der Figurenkonstellation korrelieren. (2) In Texten bzw. Textsegmenten, die bestimmte Erzählkerne bzw. Erzählschemata repräsentieren, könnte eine spezifische Figurenkonstellation vorliegen. Wenn beide Thesen zutreffend sind, könnte die Netzwerkanalyse ein geeignetes Analyseinstrument für Erzählkerne bzw. Erzählschemata sein.

Untersucht werden je drei Vertreter des Brautwerbungsschemas (kurz: BW), des Mahrteneheschemas (ME) und der Freier-Befreiungsgeschichte (FB). ${ }^{13}$ In der Freier-Befreiungsgeschichte wird eine alleinstehende Burgherrin von einem Freier belagert, der sowohl die Hand als auch die Herrschaft der Dame gewinnen will; sie lehnt die Verbindung jedoch ab und lässt ihre Burg - meist mehr schlecht als recht vor dem Angreifer verteidigen. Ein junger Ritter kommt als Retter dazu, er besiegt den Freier und darf die Burgherrin heiraten. Bei Mahrtenehe-Erzählungen kommt ein junger Ritter in eine Anderwelt, etwa auf eine Insel, und trifft dort eine Fee. Neben der erotischen Initiation hilft die Fee ihrem Geliebten, als Ritter erfolgreich zu sein. Ihre Bedingung für die Beziehung ist, dass der Ritter ein Tabu (Sehtabu, Namenstabu, ...) einhält; nach dem obligatorischen Tabubruch folgt das endgültige Ende der Beziehung oder ein Bewährungsweg. ${ }^{14}$ Beim Muster der gefährlichen Brautwerbung muss ein Brautwerber mit Hilfe eines Werbungshelfers den Widerstand des Brautvaters überwinden, um die Braut zu gewinnen. ${ }^{15}$ Ein Problem für eine digitale Auswertung liegt darin, dass mittelalterliche Texte nur selten ein Schema und eine Gattung in Reinform repräsentieren - häufig findet man Interferenzen und Gattungs-

10 Vgl. Lena Hettinger u. a., Genre classification on German novels, in: 26th International Workshop on Database and Expert Systems Applications (DEXA), 2015, 249-253.

11 Grundlegend zu Erzählschemata Armin ScHULz, Erzähltheorie in mediävistischer Perspektive. Herausgegeben von Manuel Braun / Alexandra Dunkel / Jan-Dirk Müller, Berlin / Boston 2012, 159-191.

12 Vgl. zum Begriff Jan-Dirk MüLLER, Höfische Kompromisse. Acht Kapitel zur höfischen Epik um 1200, Tübingen $2007,22$. Während ein Erzählschema in der Regel einem ganzen Text oder doch großen Teilen zugeschrieben wird, können Erzählkerne auch als kleinere Einheiten beschrieben werden.

13 Die Freier-Befreiungsgeschichte wäre bei einer engen Verwendung des Begriffs „Erzählschema“ eher als Erzählkern einzustufen, da sie meist in einen größeren Kontext eingebettet ist und etwa als eine Einheit in episodisch organisierten Texten vorliegt.

14 Zur Mahrtenehe-Erzählung vgl. ScHuLz, Erzähltheorie, 214-231.

15 Zum Brautwerbungsschema vgl. Christian SCHMID-CADALBERT, Der, Ortnit AW' als Brautwerbungsdichtung. Ein Beitrag zum Verständnis mittelhochdeutscher Schemaliteratur, Bern 1985 (Bibliotheca Germanica 28), 80-100; ScHULZ, Erzähltheorie, 204-207. 
hybride ${ }^{16}$ : So lässt sich Gottfrieds „Tristan“ kaum nur auf das Brautwerbungsschema reduzieren; die hier untersuchten Brautwerbungs-Erzählungen gehören zudem verschiedenen Gattungen an, wobei die Gattungszuschreibungen bzw. Gattungsbegriffe selbst umstritten sind. (Eine mögliche Zuschreibung wäre: „Tristan“ - höfischer Roman, „Kudrun“ - Heldenepos, „König Rother“ - Spielmannsepik.)

Um dieser Problemlage zu begegnen - und auch aus Gründen der Annotationszeit - wurden nicht die vollständigen Texte annotiert, sondern die Passagen, die am ehesten dem jeweiligen Erzählschema zugeordnet werden können:

\begin{tabular}{|l|l|}
\hline & \\
\hline BW & König Rother 1-2966 \\
\hline BW & Tristan (8226-12614, Werbung um Isolde bis zur Hochzeitsnacht) \\
\hline BW & Kudrun (204,1-567,4, Werbung um Hilde) \\
\hline FB & Parzival (4,27-58,26, Belakane) \\
\hline FB & Parzival (179,13-223,30, Condwiramurs) \\
\hline FB & Gregorius (1838-2243) \\
\hline ME & Gauriel (36-868 + 2560-2949) \\
\hline ME & Ritter von Staufenberg 1-1192 \\
\hline ME & Melusine, ed. Müller, 13,3-49,14, 92,13-126,34 (weitgehend ohne Söhne-Handlung) \\
\hline
\end{tabular}

\section{Annotation}

Annotiert wird direkt in die jeweiligen Textdateien. Erneute Figurenreferenzen werden erst im übernächsten Vers (Prosa: innerhalb von 10 Wörtern) wieder annotiert, da Referenzen auf dieselbe Figur häufig eng beisammenstehen, ein Zugewinn für die Auswertung der Nähe von zwei Figurenreferenzen wäre durch die mehrfache Annotation jedoch marginal. Allerdings wird eine weitere Referenz auf dieselbe Figur innerhalb von zwei Versen dann annotiert, wenn eine Referenz auf eine andere Figur zwischen den beiden Referenzen auf dieselbe Figur steht. Figurengruppen wie etwa die Grafen im „König Rother" werden als Gruppe wie einzelne Figuren annotiert - dabei handelt es sich um eine Setzung; diese Entscheidung könnte auch anders getroffen werden. Dagegen werden funktional ähnliche, aber einzeln benannte Figuren wie die Werbungshelfer in der „Kudrun“ (Fruote, Wate, Morung) nicht zusammengefasst, sondern einzeln annotiert. Eine weitere Setzung betrifft Fälle, in denen teils einzelne Vertreter einer Gruppe und andererseits auch die Gruppe als Kollektiv agieren: Hier wird darauf verzichtet, das Handeln zu vereinheitlichen; im „Staufenberg“ werden ,Pfaffheit“ und Bischof einzeln erfasst. Ganz unkonkrete, singuläre Referenzen im Sinn von ,jedermann“ werden nicht annotiert (sie haben ohnehin keine Aussicht, zu den zehn häufigsten Figuren bzw. Figurengruppen zu zählen, die hier untersucht werden): „Iwein“, V. 45f.: ,in <Artus> liebte hof und den lîp / manec maget unde wîp [ohne Tag]". Wenn etwa ein Pronomen im Plural auf zwei Figuren referiert, werden beide Figuren annotiert.

Bei der Figurenrede wird nur zwischen gesprochener und innerer Figurenrede differenziert, nicht jedoch nach direkter oder indirekter Rede; zudem wird die betreffende Figur erfasst. Innere Figurenrede umfasst demnach direkte innere Rede (innerer Monolog), indirekte innere Rede (Gesprächsbericht) und Bewusstseinsdarstellung (Emotionen, Wahrnehmungen wie sehen/hören und auch diffuse Be-

16 Vgl. exemplarisch Stephan Fuchs, Hybride Helden: Gwigalois und Willehalm. Beiträge zum Heldenbild und zur Poetik des Romans im frühen 13. Jahrhundert, Heidelberg 1997 (Frankfurter Beiträge zur Germanistik). 
schreibungen innerer Zustände). ${ }^{17}$ Verba dicendi werden bei direkter Rede nicht mit in die annotierte Passage eingeschlossen, bei indirekter Rede jedoch schon mit ausgezeichnet. ${ }^{18}$ Bei Figurenreden, die zwei Sprecher haben, werden in doppelter Annotation beide Sprecher erfasst und mit nachgestelltem <gr_multi>-Tag versehen, um Auswertung und Syntaxcheck zu erleichtern. Geschachtelte Figurenreden werden nur dann ausgezeichnet, wenn es sich um ,ausgewachsene“ Binnenerzählungen handelt (mehr als 10 Verse). Ansonsten wird nur die ,äußere' Rede annotiert. Spezialfälle wie sie Annelen Brunner mit Hilfe von „@non-fact“, „@border“, „@ narr“ erfasst, werden nicht annotiert. ${ }^{19}$ Fragmente einer indirekten inneren Figurenrede, die weniger als drei Wörtern umfasst, werden nicht annotiert, wenn dabei ohnehin keine Referenz auf eine andere Figur vorliegt. Nicht als innere Figurenrede annotiert würde demnach: „Er ging traurig weiter“ (fiktives Beispiel). Annotiert würde jedoch: „Er dachte traurig an Georg“ oder „Er hörte Georg“.

\section{Auswertung}

Ein erstes Perl-Skript sucht Tags und ermittelt ihre Häufigkeit, womit Tippfehler ermittelt werden können. Ein erster Syntaxcheck gleicht die öffnenden und schließenden Tags bei Redewiedergabe miteinander ab. Eliminiert werden Tags wie Rezipient, Erzähler, Teufel, Maria (als Anrufungsinstanz); zudem wird eine Namensliste für die Netzwerkanalyse mit den zehn häufigsten Figuren geschrieben.

Ein zweites Perl-Skript ermittelt für die zehn häufigsten Figuren die jeweils kürzesten Distanzen zwischen je zwei Figurenreferenzen in Worten. Da beim vorliegenden Korpus ein Normalisierungs-Problem besteht - es liegen verschieden lange Texte vor, wodurch verschieden häufige Figurenokkurrenzen bedingt sind, wird versucht, dieser Problemlage mit einer doppelt ternären Gewichtung der Distanzen zu den zehn häufigsten Figuren zu begegnen.

Die erste ternäre Gewichtung 1 beruht auf drei Schwellen (bspw. 10-20-30 Wörter). Eine Distanz zwischen zwei Figurenreferenzen, die kleiner ist als die Schwelle 1 (bspw. 10 Wörter), erhält die Gewichtung „3“. Eine Distanz, die kleiner ist als die Schwelle 2 (bspw. 20 Wörter), erhält die Gewichtung „2“. Eine Distanz, die kleiner ist als die Schwelle 3 (bspw. 30 Wörter), erhält die Gewichtung „1“.

Die zweite ternäre Gewichtung bezieht die Anzahl der nahen Referenzen von zwei Figuren ein: Wie viele Distanzen mit den Distanzgewichtungen 1, 2 oder 3 zwischen den Figuren A und B wurden gefunden? Hier werden die drei höchsten Distanzgewichtungen summiert. Wenn beispielsweise für die Figuren $A$ und $B$ zwei nahe Distanzen gefunden wurden, die einmal die Gewichtung 3 und einmal die Gewichtung 1 erhalten haben, beträgt die Gesamtgewichtung der Relation 4. Wenn beispielsweise für die Figuren $C$ und D vier nahe Distanzen gefunden wurden, die einmal die Gewichtung 3 und zweimal die Gewichtung 2 und einmal die Gewichtung 1 erhalten haben, beträgt die Gesamtgewichtung der Relation 3+2+2=7. Der Maximalwert beträgt 9. Denkbar wäre, bei diesem Verfahren noch einen Faktor zu verwenden, damit eine höhere Anzahl an Distanzgewichtungen eingehen muss, bis die Gesamtgewichtung steigt.

\footnotetext{
17 Vgl. Gert HüBNER, Erzählform im höfischen Roman. Studien zur Fokalisierung im ,Eneas', im ,Iwein“ und im ,Tristan‘, Tübingen 2003 (Bibliotheca Germanica 44), 47.

18 In Anschluss an Annelen BRUNNER, Automatische Erkennung von Redewiedergabe. Ein Beitrag zur quantitativen Narratologie, Berlin / Boston 2015 (Narratologia 47), 66f.

Vgl. BRUNNER, Redewiedergabe, 72ff.
} 
Für die zehn häufigsten Figuren wird der Degree der jeweiligen Figuren und sowie der Gesamtdegree berechnet. Der Degree ist ein Zentralitätsmaß, das aus der Anzahl der Beziehungen einer Figur besteht. Weiterhin wird der Closeness-Wert für die Figuren sowie der Durchschnitt dieser Closeness-Werte berechnet. Closeness ist ein Zentralitätsmaß, das misst, wie lang die Wege im Durchschnitt sind, über die eine Figur alle anderen Figuren im Netzwerk erreichen kann: Wie nah liegt ein Akteur an den anderen Akteuren? ${ }^{20}$ Ein Python-Networkx-Skript berechnet die Betweenness-Zentralität aller Figuren und den Mittelwert dazu und gibt eine DL-Datei der Affiliationsmatrix für den UCInet-Import aus. Betweenness ist ein Zentralitätsmaß, das misst, wie viele kurze Verbindungen zwischen 2 Akteuren über einen Akteur in der Mitte laufen. ${ }^{21}$ „Personen, die zwei ansonsten unverbundene Teilpopulationen miteinander verbinden, sind typischerweise Akteure mit hoher betweenness centrality." 22

Für die Redewiedergabe muss das Verfahren nur leicht modifiziert werden: Ein Perl-Skript sucht zu jeder Figur den Text, der von Redewiedergabe-Tags umschlossen ist, und zählt darin die Figurenreferenzen auf alle Figuren. Eine gerichtete Beziehung von Figur A zu Figur B wird dann erfasst, wenn die Redewiedergabe von Figur A eine Referenz auf Figur B enthält. Als Gewichtung wird die Anzahl der Referenzen von Figur A auf Figur B verwendet (normalisiert: geteilt durch die Anzahl der Beziehungen im Text insgesamt). Die Berechnungen erfolgen wie bei den Distanzen; hier jedoch mit Indegree und Outdegree (gewichtet): Outdegree ist ein Zentralitätsmaß, das erfasst, wie viele gerichtete Beziehungen zu anderen Figuren von Figur A ausgehen. Indegree ist ein Zentralitätsmaß, das erfasst, wie viele gerichtete Beziehungen von anderen Figuren bei Figur A eingehen.

\section{Vergleichbarkeit von Figuren und Aktanten}

Ich verwende hier Aktantenlogik nach Greimas in der pointierten Darstellung von Warning, um Figurentypen einander zuzuordnen. ${ }^{23}$ Demnach wird operiert mit einem Helden, dem Subjektaktanten, einem Opponenten, der besiegt werden muss, einer Helferfigur (Adjuvant), und einem Objektaktanten - bspw. im Brautwerbungsschema die Braut, die der Brautwerber gerne erwerben möchte. Eine Zuordnung ist nicht immer ganz unproblematisch: In der „Kudrun“ handeln die Werbungshelfer mehr als der Brautwerber, so dass man auch überlegen könnte, ob Hetel tatsächlich Subjektaktant ist. In MahrteneheErzählungen ist die Bestimmung des Opponenten nicht unproblematisch: Wohl wichtiger als Zweikampf-Gegner sind die Figuren, die den Helden zum Tabubruch verführen, doch dabei handelt es ich meist um Freunde oder enge Verwandte, also gerade nicht um die klassischen Bösewichte. Die Position des Adjuvanten ist in Mahrtenehe-Erzählungen nicht unbedingt besetzt oder identisch mit der weiblichen Hauptfigur.

20 Vgl. Mark Trappmann / Hans J. Hummell / Wolfgang Sodeur, Strukturanalyse sozialer Netzwerke. Konzepte, Modelle, Methoden, Wiesbaden 2005, 46-51.

21 Vgl. Dorothea JANSEN, Einführung in die Netzwerkanalyse, Opladen 2003 (UTB 2241), 141.

22 TRAPPMANN / HUMmELL / SODEUR, Strukturanalyse sozialer Netzwerke, 52.

23 Algirdas Julien GreIMAS, Strukturale Semantik. Methodologische Untersuchungen. Autorisierte Übersetzung aus dem Französischen von Jens Ihwe, Braunschweig [1966] 1971 (Wissenschaftstheorie, Wissenschaft und Philosophie 4); Rainer WARNING: Formen narrativer Identitätskonstitution im höfischen Roman, in: Odo Marquard / Karlheinz Stierle, Hg.: Identität, München 1979 (Poetik und Hermeneutik 8), 553-589. 


\section{Netzwerkgraphen und Zentralitätsmaße}

Die Netzwerkgraphen wurden mit UCInet erstellt. ${ }^{24}$ Kanten und Knoten sind nach Betweenness gewichtet. Die Brautwerbungs-Erzählungen sind recht homogen. Der ,Gauriel' ähnelt etwas den Brautwerbungs-Erzählungen, er ist jedoch nicht ganz so stark verbunden. Eine klare Differenzierung zwischen Mahrtenehe-Erzählungen und Freier-Befreiungs-Erzählungen ist nicht erkennbar. Die Differenzierung zwischen Haupt- und Nebenfiguren beim „Gregorius“ ist besonders deutlich ausgeprägt.

Bei Redewiedergabe wird erfasst, welche Figur auf welche andere Figur Bezug nimmt, es handelt sich also um gerichtete Beziehungen. Rot sind einseitige Beziehungen dargestellt (Figur A erwähnt Figur B, aber nicht umgekehrt), blau sind gegenseitige Beziehungen dargestellt.

Auch hier sind die Brautwerbungs-Erzählungen auffällig homogen: Sie sind wieder stark verbunden, es gibt multiple Bezugnahmen. Im „Gregorius“ ist der Textanteil, der als Redewiedergabe vorliegt, recht niedrig. Hier deutet sich ein Problem an: Das gleiche Erzählschema kann mit mehr oder weniger Figurenrede erzählt werden. In welchem Umfang Figuren andere Figuren in Figurenrede erwähnen, muss nicht zwingend mit dem Schema zusammenhängen. Denkbar wäre immerhin, dass es gewisse Präferenzen bei bestimmten Erzählmodellen geben könnte.

Wiederum differieren Mahrtenehe-Erzählungen und Freier-Befreiungs-Erzählungen schwächer als Brautwerbungs-Erzählungen. Die Plots ermöglichen viele interessante Einzelbeobachtungen, die aus Raumgründen hier nicht ausgebaut werden können. Exemplarisch sei auf die zahlreichen einseitigen Beziehungen in den Mahrtenehe-Erzählungen verwiesen; die Beziehung zwischen Hauptfigur und Fee sticht hier heraus. Weiterhin ist in der „Kudrun“ die Braut Hilde stark zu den Werbungshelfern hin orientiert - etwa mehr zum Werbungshelfer Horand als zum künftigen Ehemann Hetel.

Distanzen 10-20-30

Freier-Befreiung: Greg, Pz Bela, Pz CWA
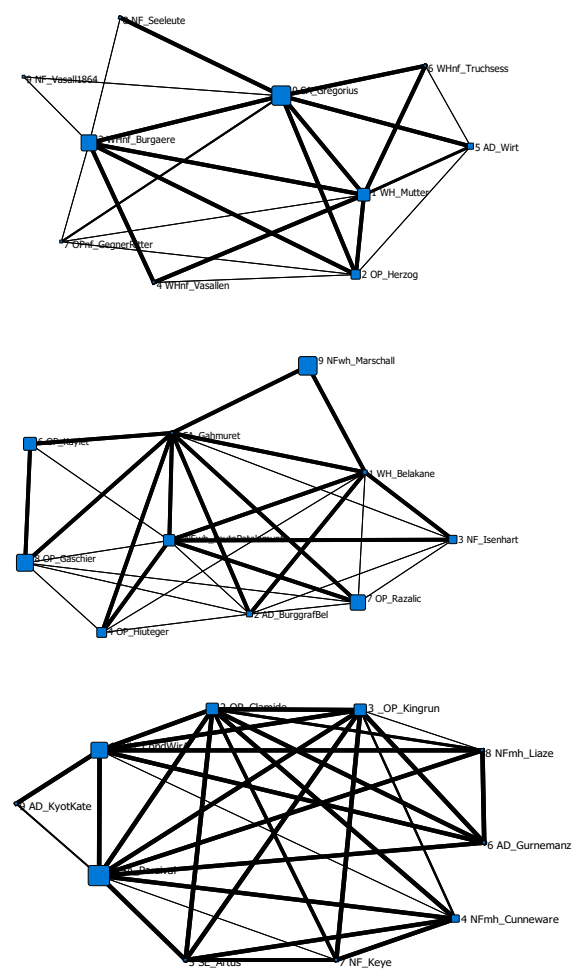

Brautwerbung: Kudr, Roth, Trist
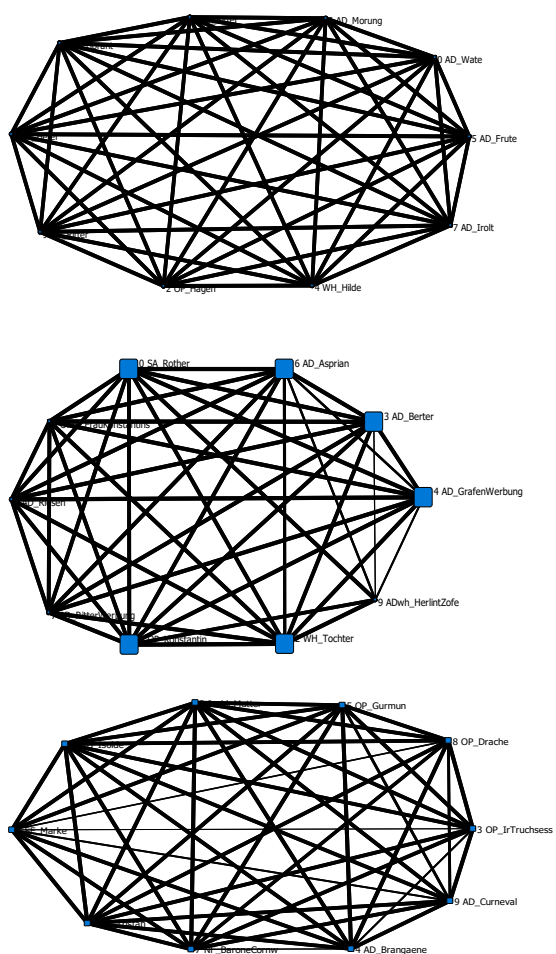

Mahrtenehe: Gau, Mel, Stau
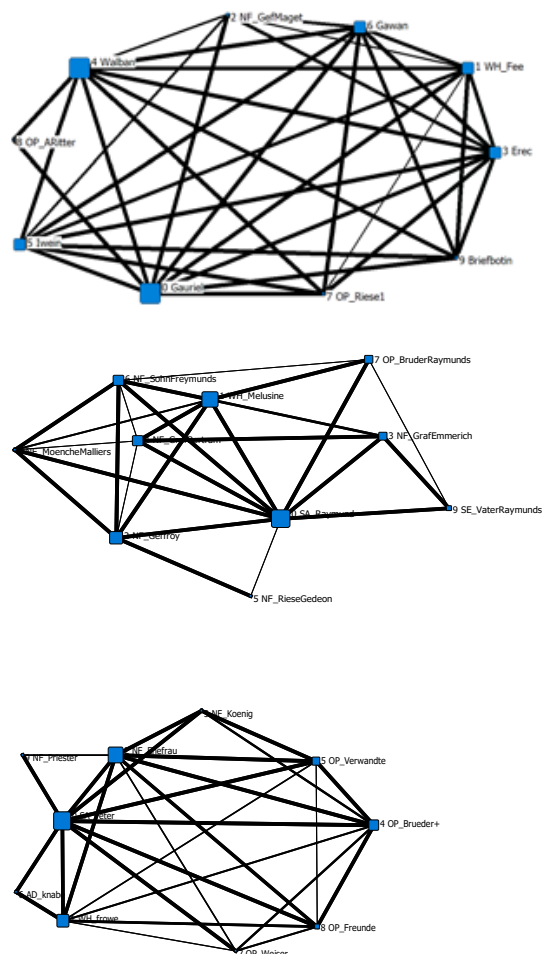

24 S.P. Borgatti / M.G. Everett / L.C. FreEmAN, Ucinet 6 for Windows: Software for Social Network AnalysiHarvard, MA: Analytic Technologies 2002. 
Freier-Befreiung: Greg, Pz Bela, Pz CWA
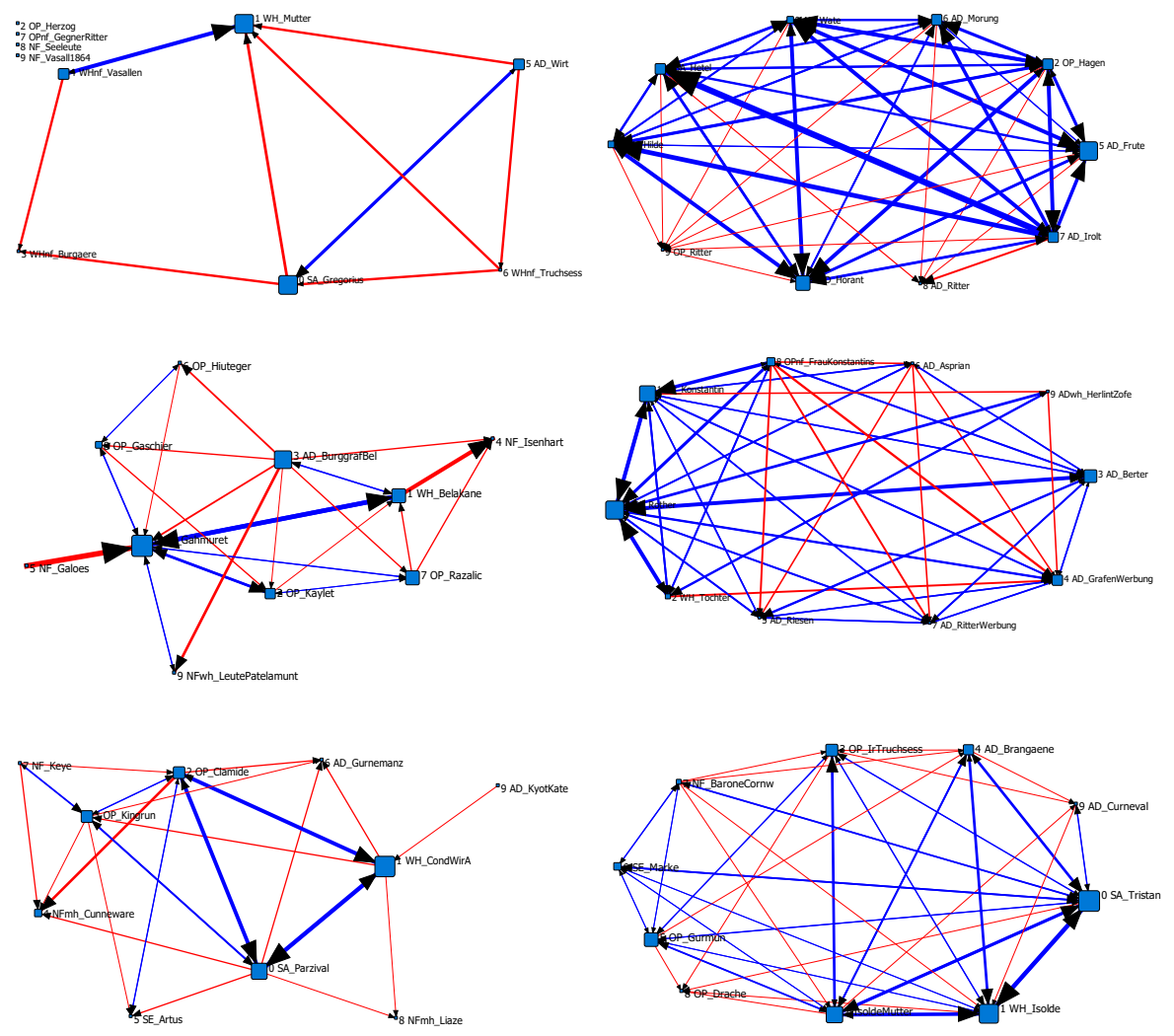

Mahrtenehe: Gau, Mel, Stau
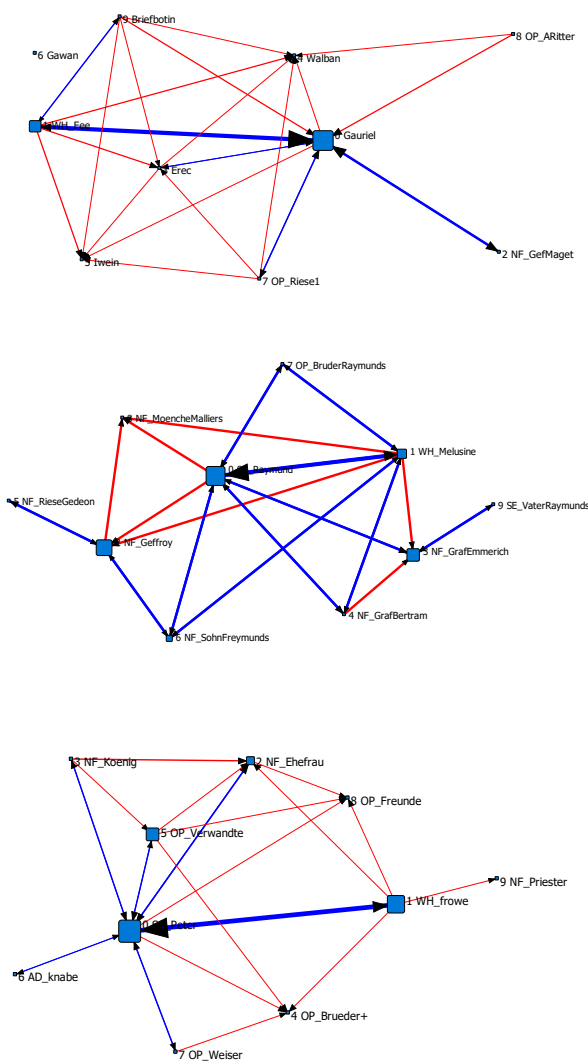

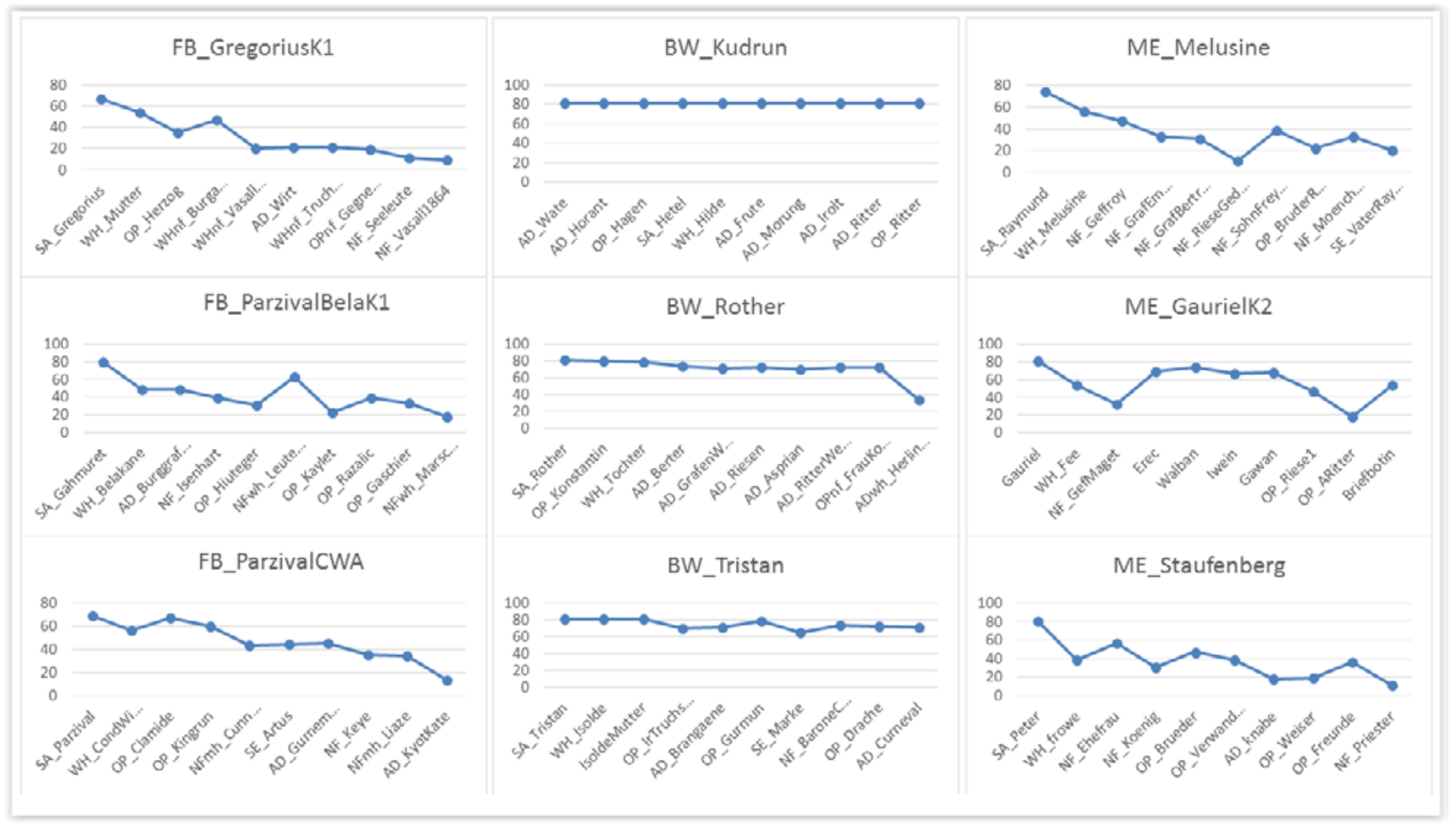

Distanzen: Degree je Text für die zehn häufigsten Figuren 


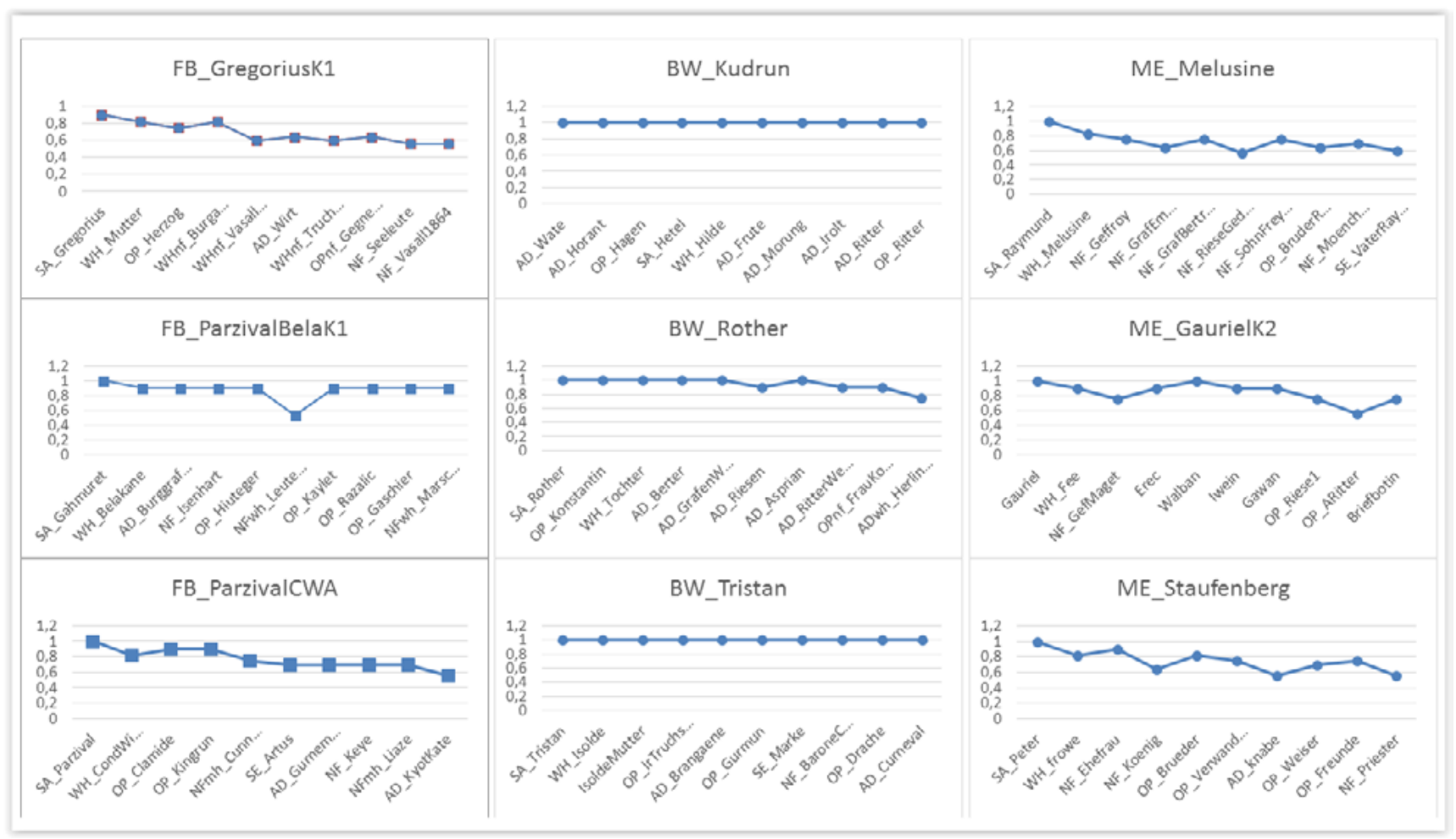

Distanzen: Closeness je Text

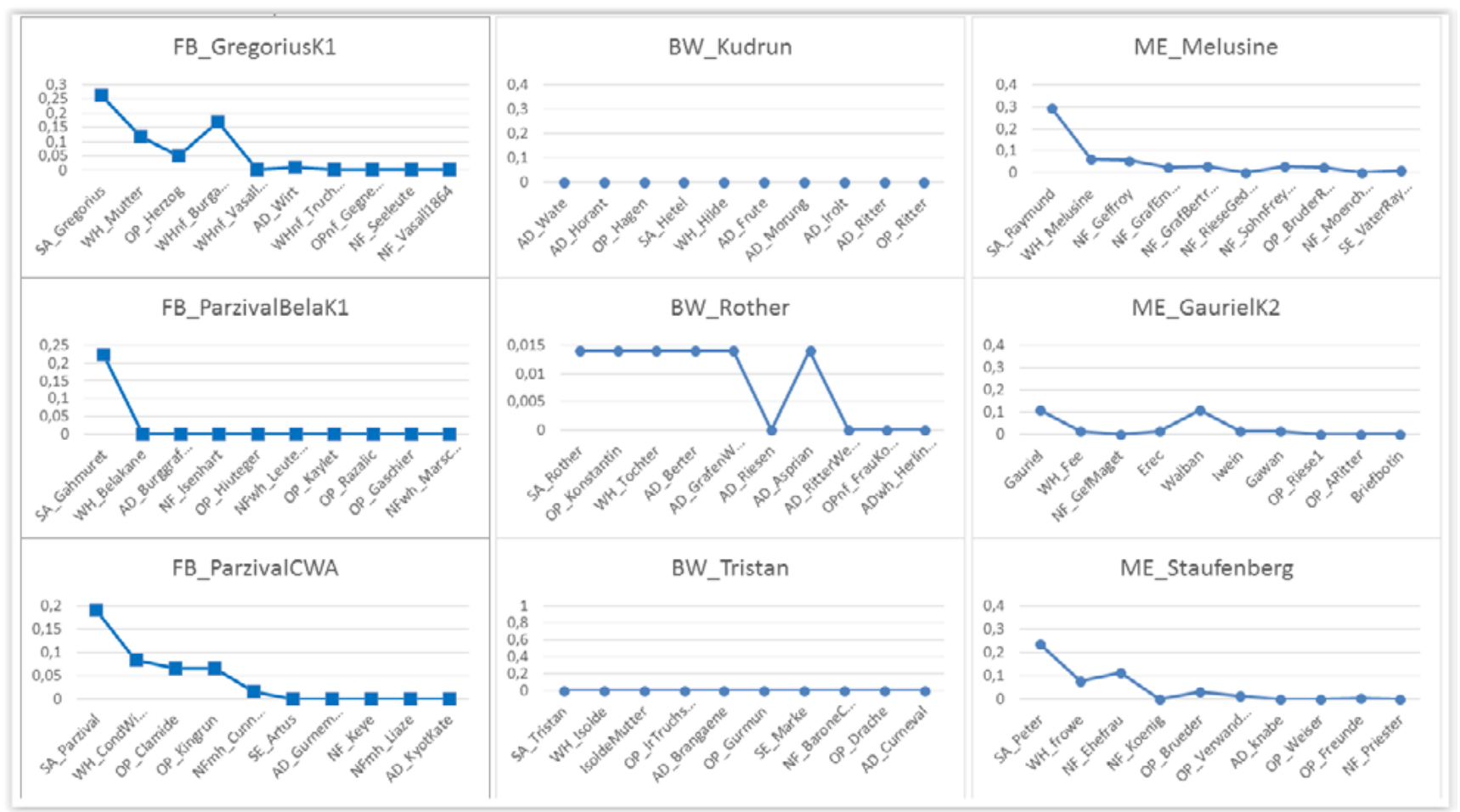

Distanzen: Betweenness je Text 
In den bisherigen Diagrammen wurden die Profile pro Text für die zehn häufigsten Figuren dargestellt. Im Folgenden werden die jeweils Mittelwerte und die Daten für Subjektaktant, weibliche Hauptfigur und Opponent für alle Texte in einem Diagramm erfasst. Die ersten drei Datenpunkte gehören jeweils zu Freier-Befreiungsgeschichten, dann folgen drei Datenpunkte zu Brautwerbungs-Geschichten, die letzten drei gehören zu Mahrtenehe-Erzählungen.

Eine gute Differenzierungsleistung ist dann gegeben, wenn je drei Punkte eines Erzählschemas gleichauf sind und zugleich noch jeweils auf einem anderen Niveau liegen wie die anderen. Das ist jedoch nur manchmal und nur annäherungsweise der Fall.
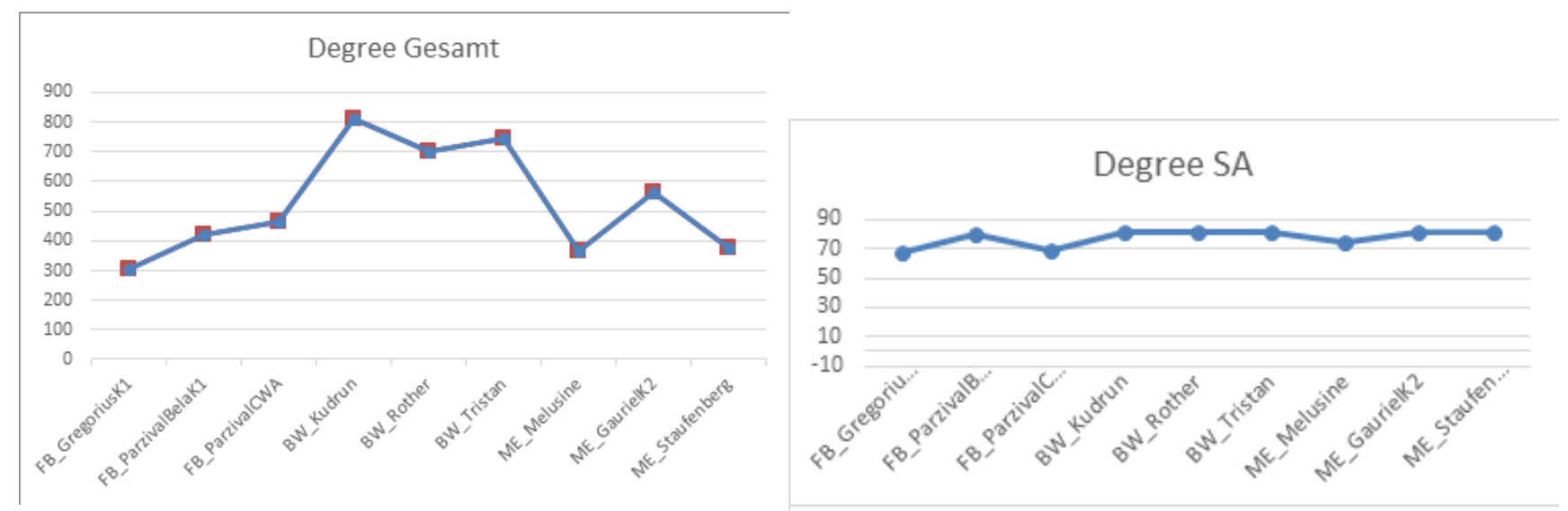

Distanzen: Degree gesamt, SA, WH, OP

Degree WH

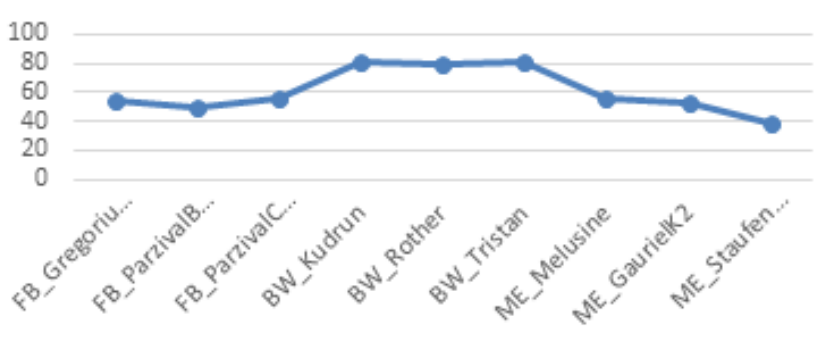

Degree OP Mittelwerte:

FB 54,0

BW 77,0

ME 38,3
Degree OP

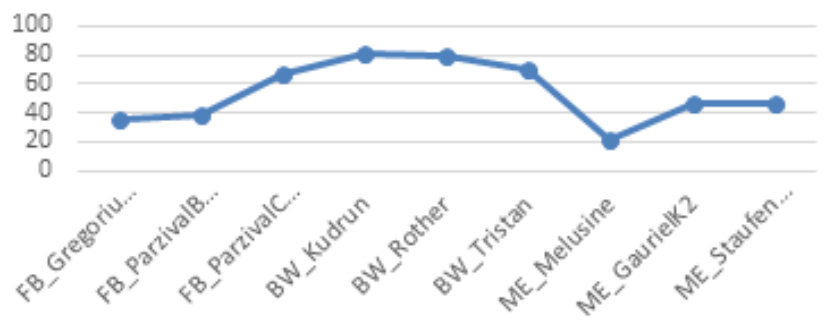




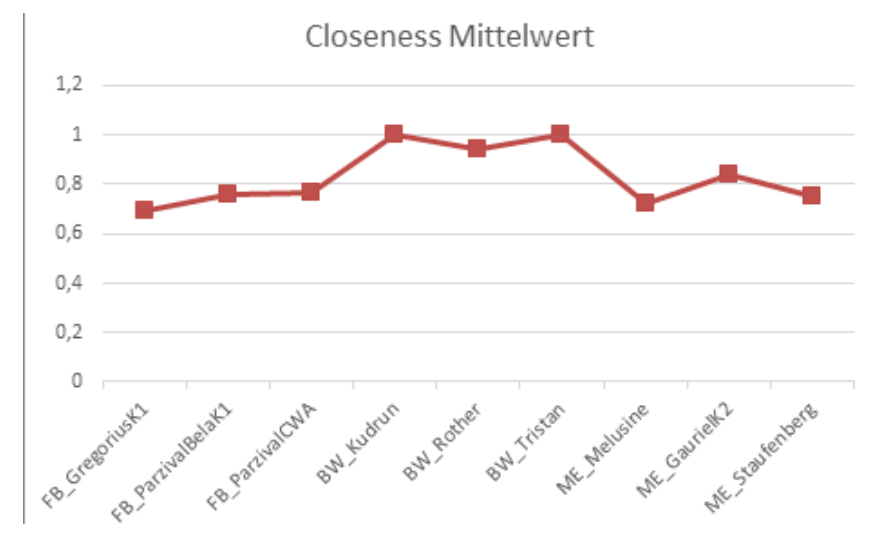

Distanzen: Closeness MW, SA, WH, OP

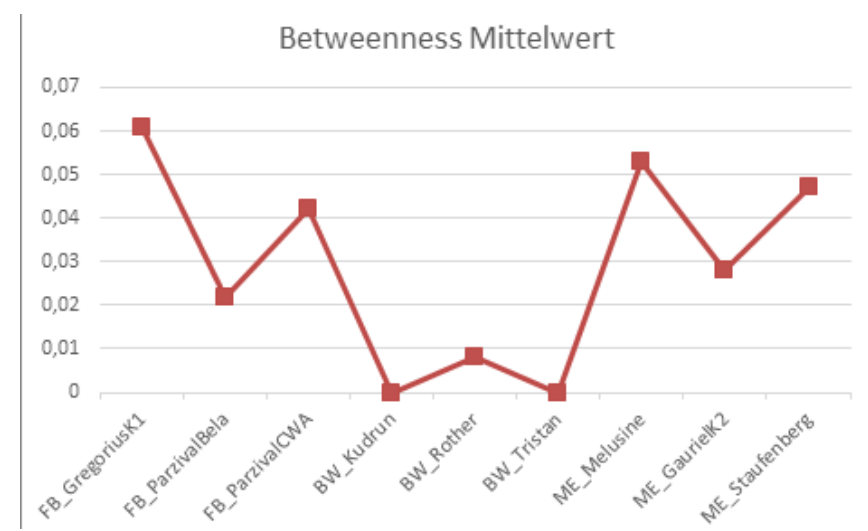

Distanzen: Betweenness MW, SA, WH, OP
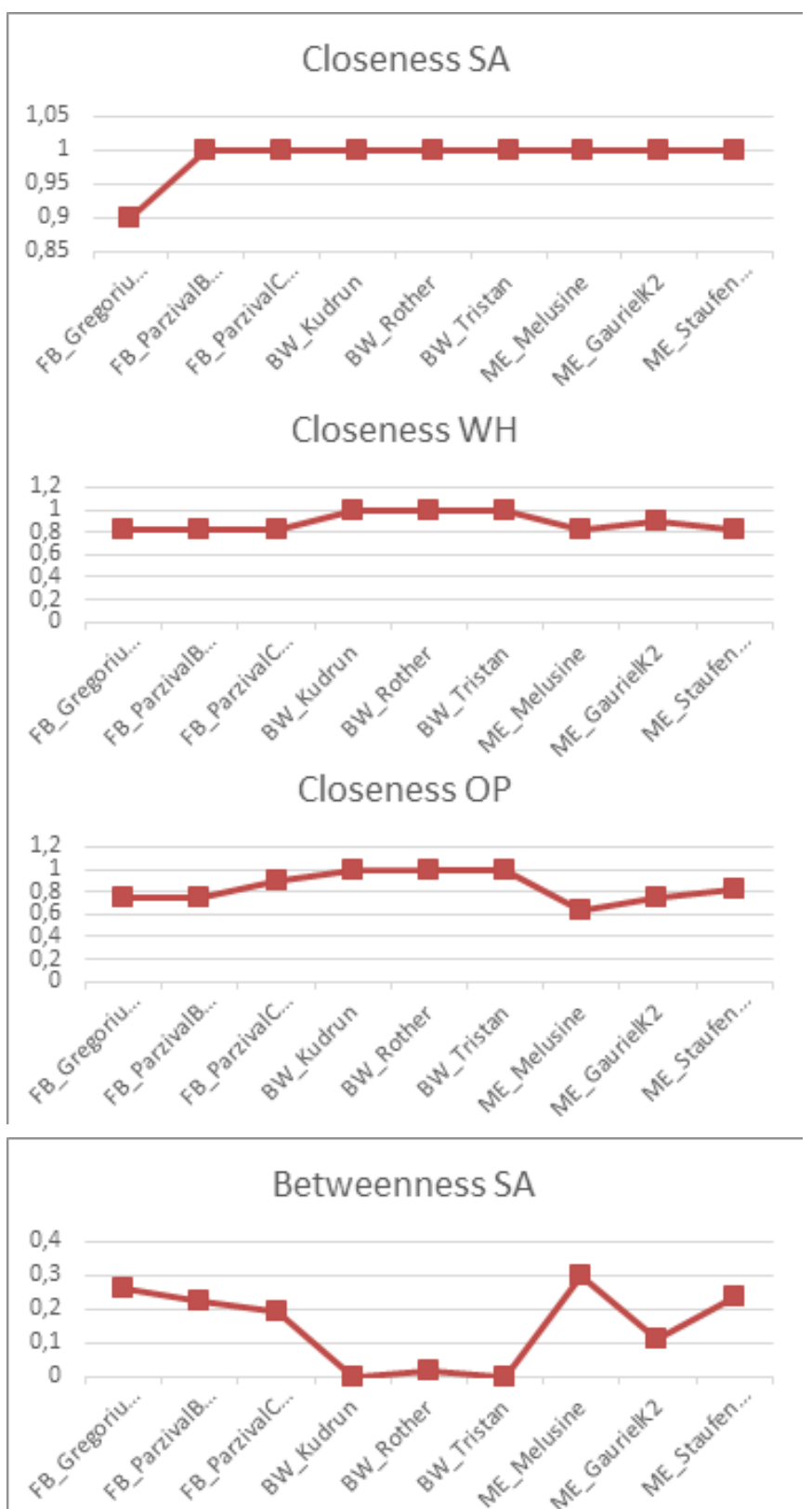

Betweenness WH

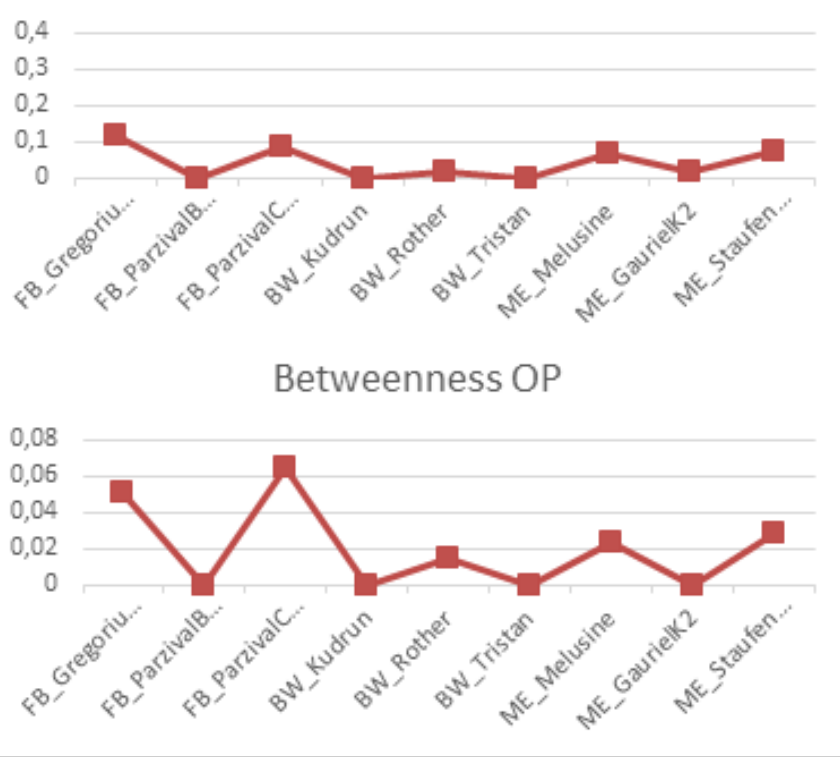




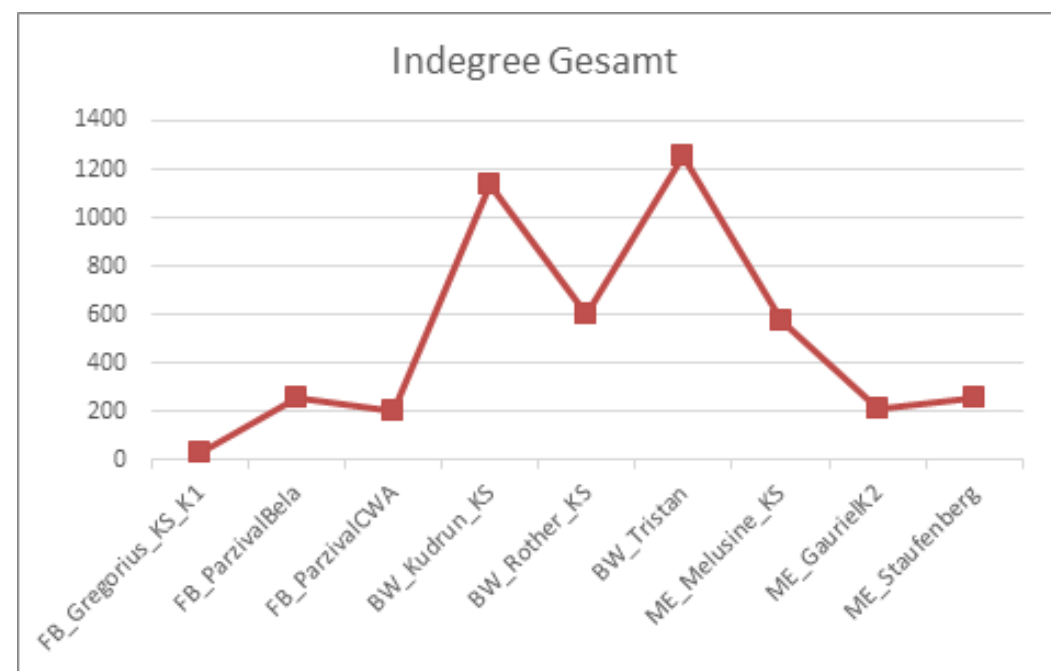

Redewiedergabe: Indegree gesamt, SA, WH, OP

Bei Degree-Werten < 1: Zahl der Referenzen der jeweiligen Redewiedergabe der Figur geteilt durch Gesamtzahl aller Referenzen Redewiedergabe
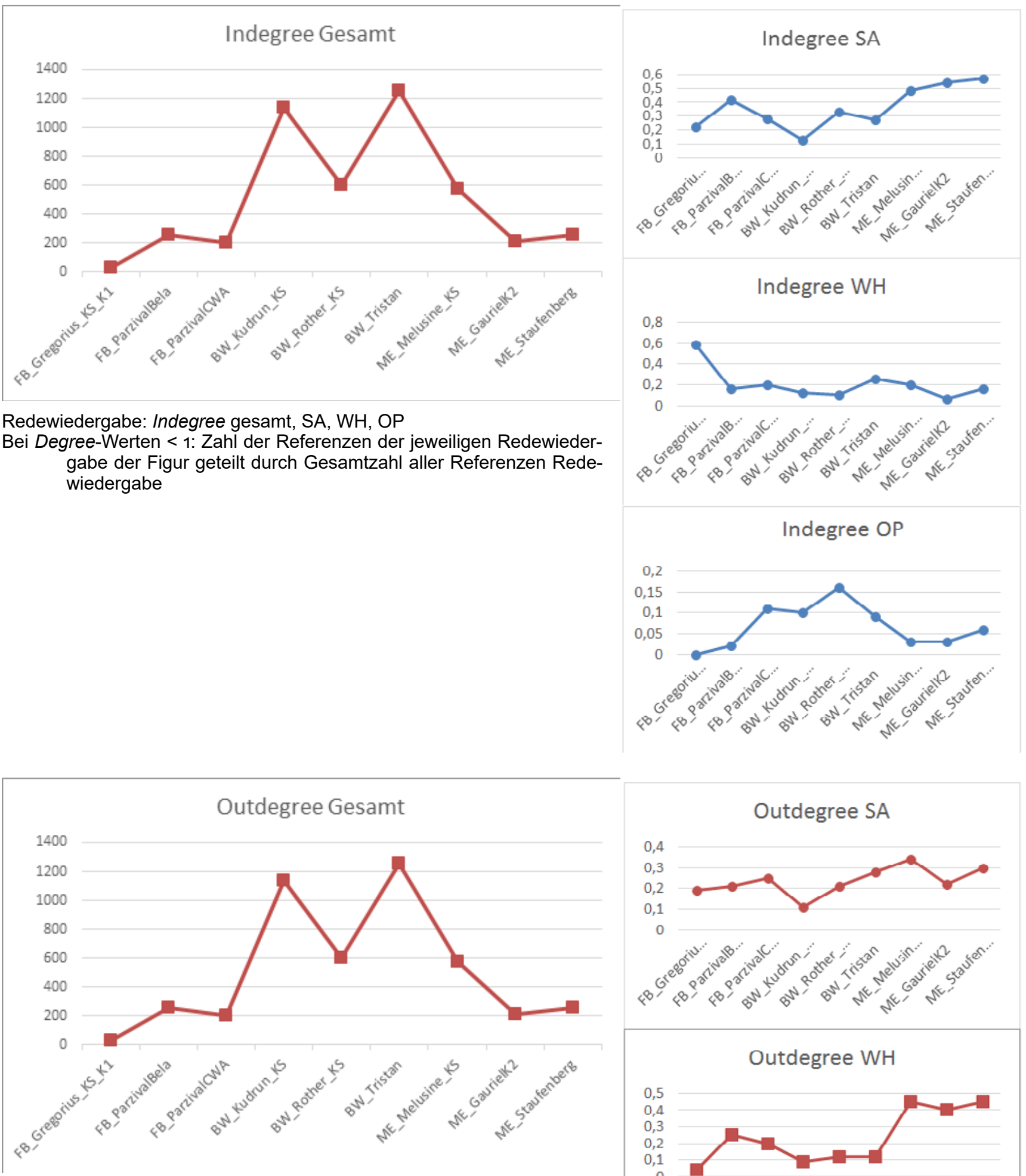

Redewiedergabe: Outdegree gesamt, SA, WH, OP

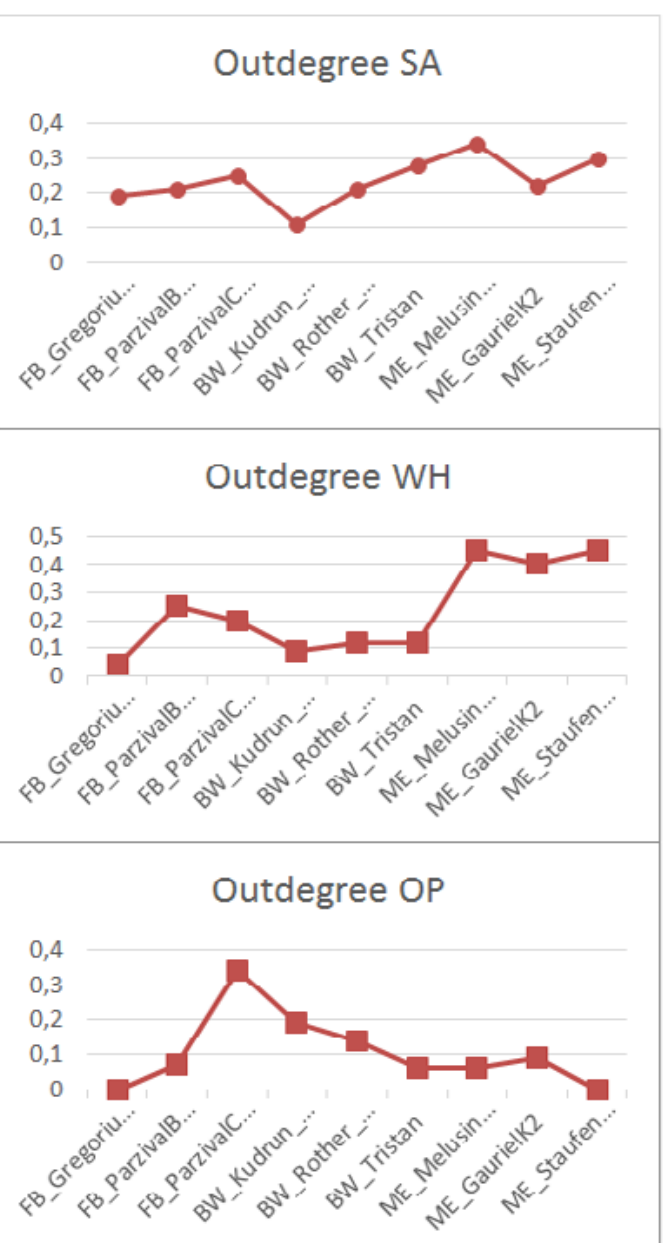


Bei dem UCInet-Network-Centralization-Index kann man besonders anhand der blauen Linie für die Distanzen nochmals die Hauptproblematik erkennen, die sich auch in einigen der vorherigen Diagramme abzeichnet: Das Niveau der jeweiligen Datenpunkte der Erzählschemata wäre dann durchaus verschieden, wenn man je Schema-Erzählung jeweils Durchschnittswerte ansetzen würde. Allerdings gibt es Ausreißer: Der „Staufenberg“ liegt im Bereich, der sonst durch die Freier-Befreiungsgeschichten abgedeckt ist. Spannend wäre es, deutlich mehr Werte zu haben, um dann beurteilen zu können, ob die Mittelwerte je Schema-Erzählung weiterhin differieren und ob die Ausreißerproblematik sich mit mehr Texten als weniger virulent erweist.

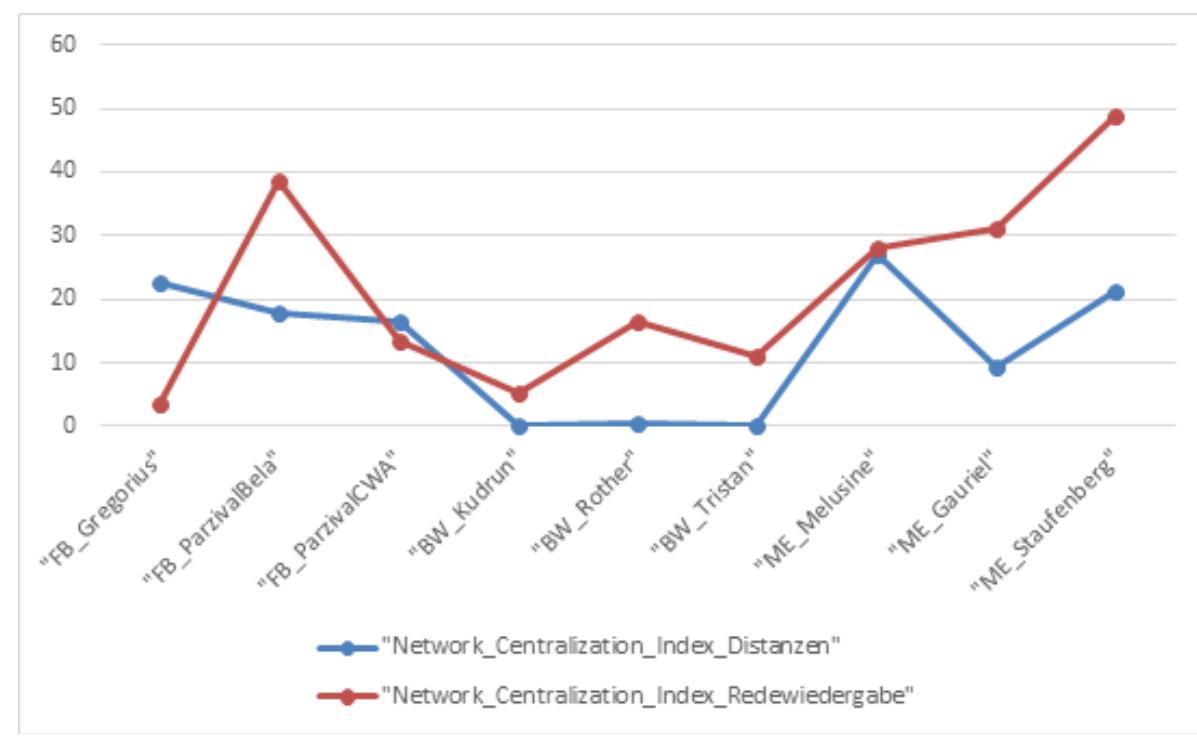

UCInet: Network Centralization Index

\section{Z-Werte und Delta-Plots für alle 29 Zentralitäts-Werte}

Hier werden für alle 29 Zentralitäts-Werte ${ }^{25}$ die jeweiligen Mittelwerte für alle Texte sowie Standardabweichung und Z-Wert berechnet.

Im Diagramm folgt hier der Durchschnitt der Z-Werte für alle Werte. ${ }^{26}$ Hier ist der „Gregorius“-Wert klar als Ausreißer erkennbar.

25 Network Centralization Index Dist, Network Centralization Index Redewiedergabe, Dist MW Degree, Dist MW Closeness, Dist MW Betweenness, Dist Degree SA, Dist Degree WH, Dist Degree OP, Dist Closeness SA, Dist Closeness WH, Dist Closeness OP, Dist Betweenness SA, Dist Betweenness WH, Dist Betweenness OP, ReWie MW Indegree, ReWie MW Outdegree, ReWie Closeness MW, ReWie Indegree SA, ReWie Indegree WH, ReWie Indegree OP, ReWie Outdegree SA, ReWie Outdegree WH, ReWie Outdegree OP, ReWie Closeness SA, ReWie Closeness WH, ReWie Closeness OP, ReWie Betweenness SA, ReWie Betweenness WH, ReWie Betweenness OP.

26 Die Werte repräsentieren die Mittelwerte der Z-Werte in ihrer Summe - anders als bei Burrows' Delta-Berechnung, bei der für jedes Feature einzeln Z-Wert-Differenzen berechnet werden. 


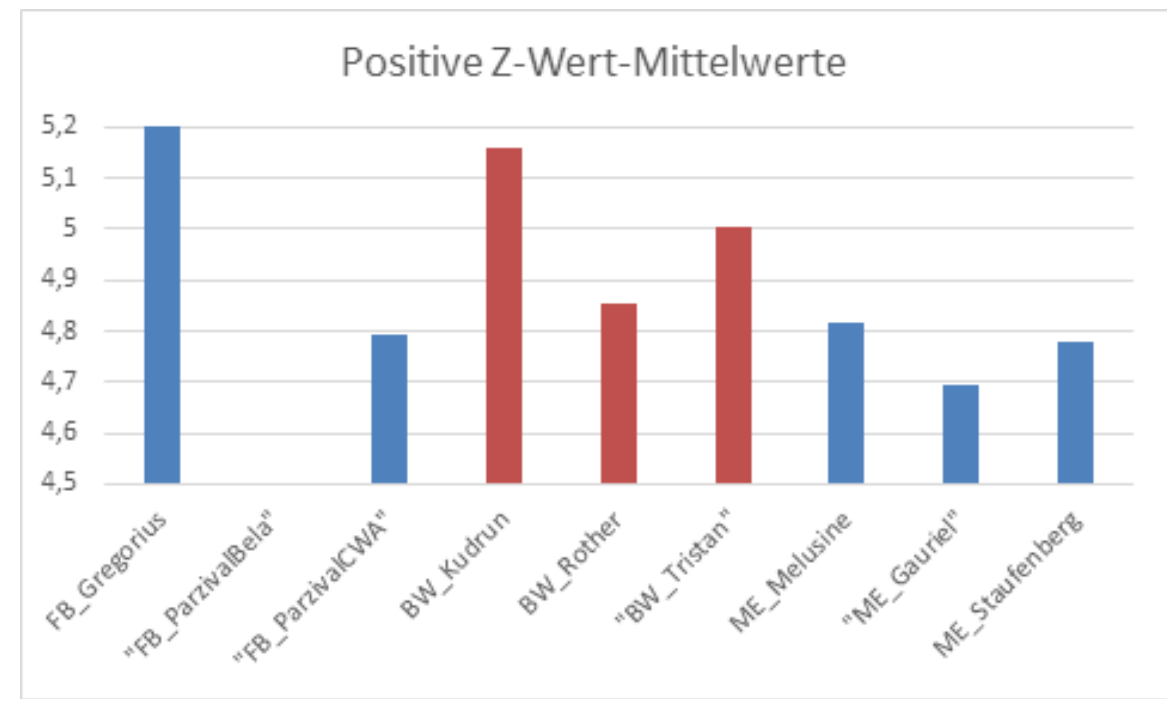

Zu diesen Zentralitäts-Werten lassen sich mit Hilfe von stylo- $R^{27}$ Delta-Distanzen nach Burrows'28 berechnen - siehe Folgeseite. Die auf den ersten Blick erfreuliche Farbsortierung täuscht, denn ,ME_ Gauriel' und ,FB_ParzivalBela' dürften bei einer optimalen Differenzierungsleistung nicht am gleichen Ast hängen. Die Distanz-Tabelle zeigt, dass die Differenzierung bei Brautwerbungs-Erzählungen sehr gut gelingt, Mahrtenehe-Erzählungen werden noch einigermaßen gut separiert, ein Fehler liegt nur beim „Gauriel“, der deutlich zu ,FB_ParzivalBela“ clustert. Die Freier-Befreiungsgeschichten clustern nicht gut.

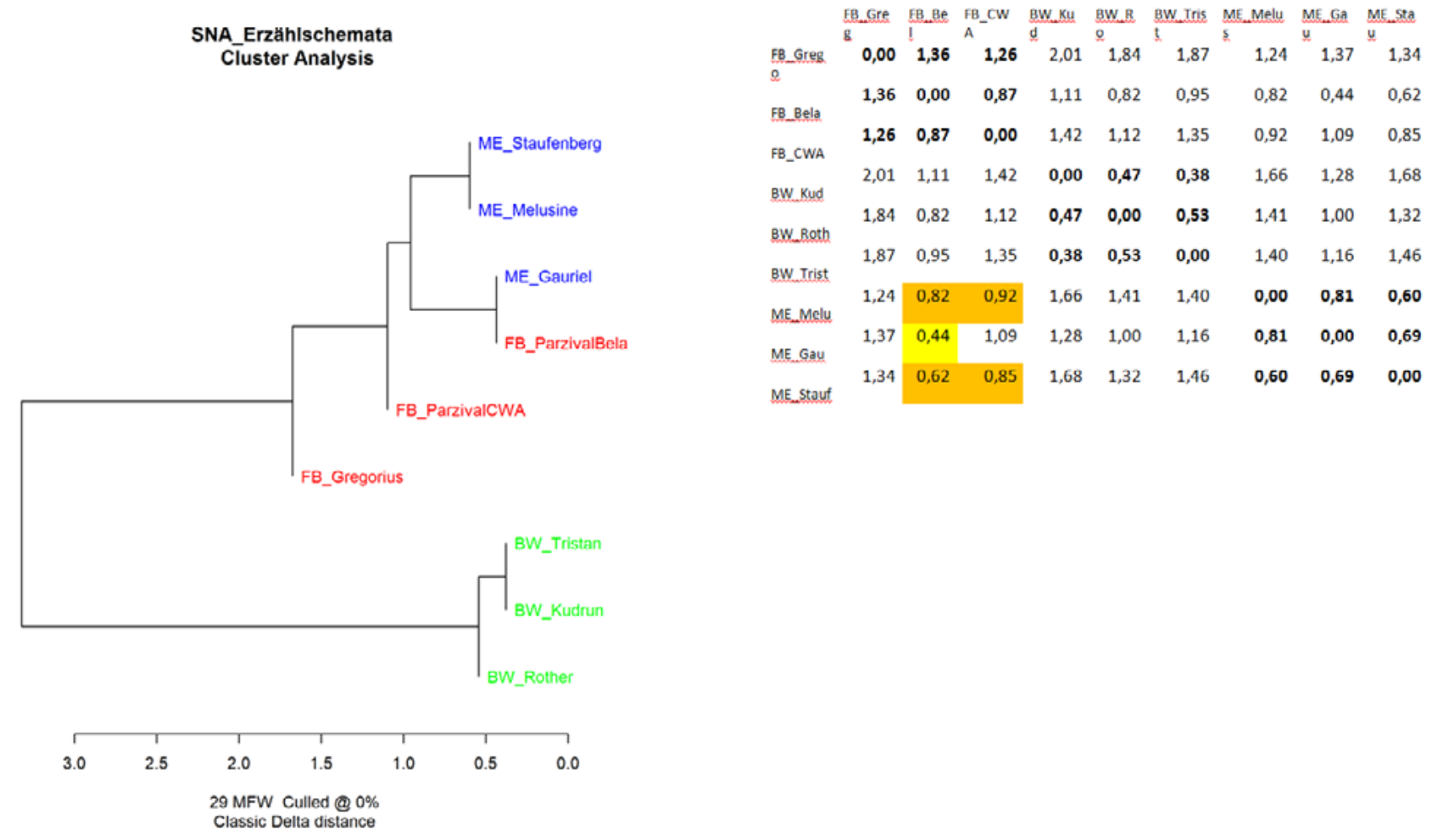

Stylo-R-Delta-Plot zu allen 29 Werten

27 Maciej EdeR / Jan RYBICKı / Mike Kestemont, stylo R package, 2017, online unter: https://sites.google.com/site/computationalstylistics/stylo (27.06.2019).

28 John F. BuRRows, ,Delta'. A Measure of Stylistic Difference and a Guide to Likely Authorship, in: Literary and Linguistic Computing 17 (2002), 267-287, DOI: 10.1093/llc/17.3.267. 
Auch wenn man bei den Mahrtenehe-Erzählungen den „Gauriel“ durch Passagen aus „Partonopier und Meliur" ersetzt, wird das Clustering schlechter. Auch hier hat man mit dem Problem zu kalkulieren, dass der Partonopier-Roman nicht nur aus einer Handlung besteht, in der das Mahrtenehe-Schema dominant spürbar wäre, sondern in Teilen kann der Text auch als Minne- und Aventiure-Roman gelten; zudem gibt es Sarazenenkämpfe wie in der Heldenepik. Armin Schulz hat ihn daher als Gattungshybrid beschrieben. ${ }^{29}$ Immerhin grenzt inn die Netzwerkanalyse eindeutig von Brautwerbungs-Erzählungen ab.

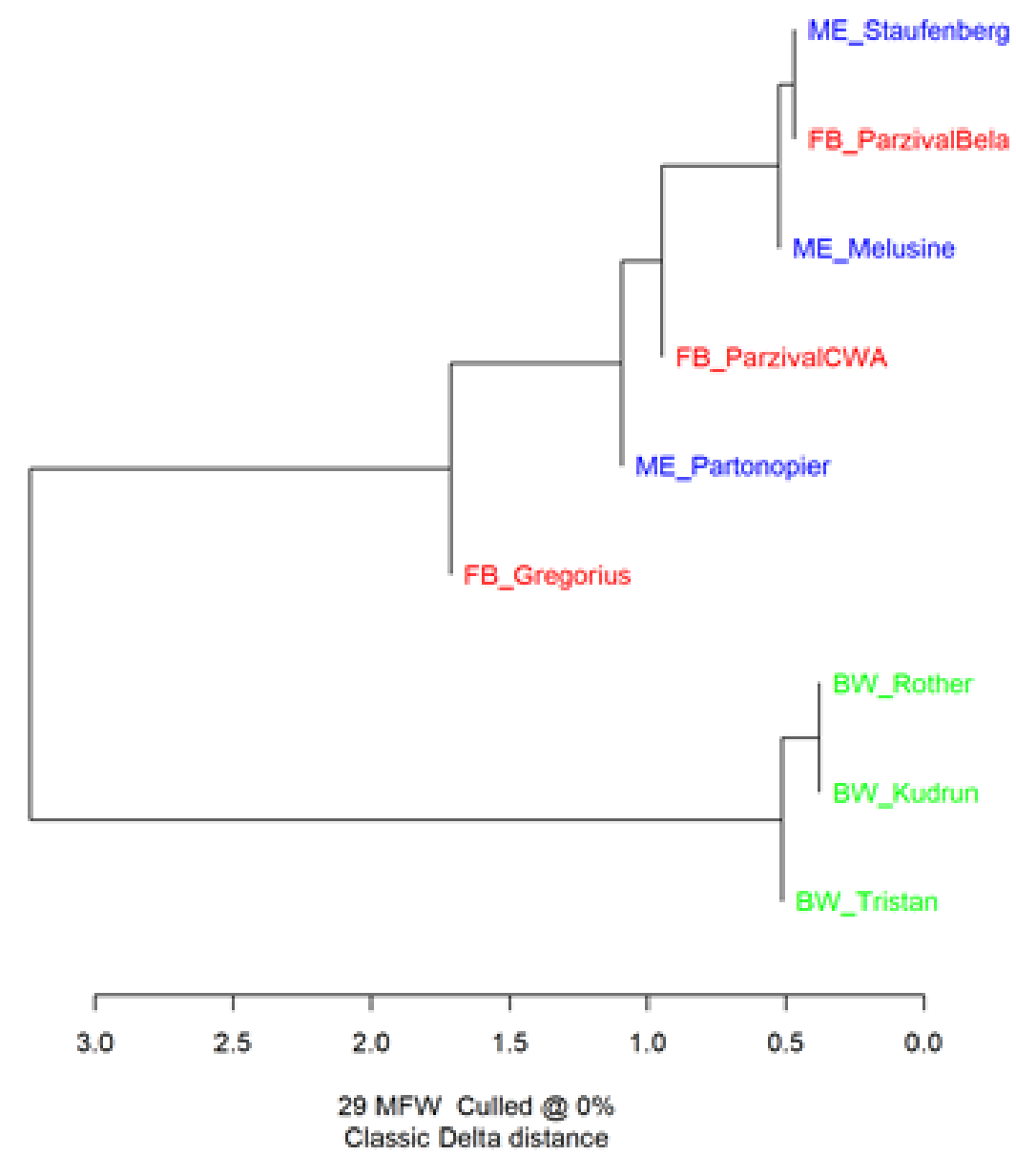

Stylo-R-Delta-Plot zu allen 29 Werten mit ,Partonopier‘ statt ,Gauriel‘

\section{Fazit}

In Bezug auf meine Ausgangsthesen fällt die Bilanz positiv aus hinsichtlich der Differenzierung der Brautwerbungs-Erzählungen von anderen Erzählschemata, die Differenzierungsleistung zwischen Freier-Befreiungsgeschichten und Mahrtenehe-Erzählungen bleibt jedoch undeutlich. Als Analyseinstrument für Erzählschemata hat die Netzwerkanalyse also zumindest ein interessantes Potential.

Notwendig wäre nun ein größeres Korpus mit weiteren Texten und weiteren Erzählkernen und Erzählschemata. Möglich wären etwa Muster wie Völkerschlacht/Heidenkrieg, Aventiuren-Schema (costume) in episodisch organisierten Romanen, Brauterwerb durch Tötung des Ehemanns oder des Vaters (Askalon, Lanzelet, Daniel), Brauterwerb im Roman durch Minnedienst (Wigalois, Gawan, ...).

29 Armin Schulz, Poetik des Hybriden. Schema, Variation und intertextuelle Kombinatorik in der Minne- und Aventiureepik: ,Willehalm von Orlens' - ,Partonopier und Meliur‘ - ,Wilhelm von Österreich“ - ,Die schöne Magelone‘, Berlin 2000 (Philologische Studien und Quellen 161), 82-121. 
Zudem könnten Schemavariationen interessant sein - etwa eine Freier-Befreiung ohne Hochzeit, wie man sie im „Iwein“ bei der Dame von Narison findet. Zudem könnte, soweit Texte vorhanden sind, ein Vergleich mit den altfranzösischen Vorlagen und mit späteren Bearbeitungen interessant sein. Anzunehmen wäre, dass sich die netzwerkanalytischen Befunde bei einer ähnlichen Makrostruktur innerhalb von Textreihen als recht homogen erweisen könnten.

Weiterhin wären weitere Experimente mit weiteren Parametern und Kennwerten durchzuführen. Interessant könnte es vor allem sein, Zeitschnitte bei den Zentralitätsmaßen zu erfassen. Wie entwickelt sich etwa die Closeness bei $30 \%$ oder $70 \%$ Handlungsfortschritt bei den verschiedenen Schemata distinkt? Eventuell könnte im Brautwerbungsschema anfangs eine enge Beziehung zwischen Werbungshelfer und Werber evident sein, weniger eng könnte anfangs die Beziehung zur Braut ausfallen. Der letzte Aspekt könnte sich bei Mahrtenehe-Erzählung umgekehrt darstellen - hier wäre zunächst eine starke Fokussierung auf Ritter und Fee zu erwarten.

Offen bleibt vorerst die Frage, ob die Redewiedergabe als Discours-Phänomen repräsentativ für Erzählschemata sein kann. Andere Probleme wie Gattungshybride dürften zentral sein; zudem bleibt das Problem virulent, in welchem Umfang sich ein Text einem Schema zuordnen lässt: Im „Staufenberg“ ist das Mahrtenehe-Schema recht stark spürbar, während sich erhebliche Teile des "Gauriel" hinsichtlich der Figurenkonstellation kaum von anderen nachklassischen Artusromanen unterscheiden. Wichtig wäre also ein Korpus mit besonders typischen Gattungsvertretern.

\section{Literaturverzeichnis}

S.P. BorgatTI / M.G. Everett / L.C. FREemAN, Ucinet 6 for Windows: Software for Social Network AnalysiHarvard, MA: Analytic Technologies 2002.

Annelen BRUNNER, Automatische Erkennung von Redewiedergabe. Ein Beitrag zur quantitativen Narratologie, Berlin / Boston 2015 (Narratologia 47).

John F. BuRRows, ,Delta'. A Measure of Stylistic Difference and a Guide to Likely Authorship, in: Literary and Linguistic Computing 17 (2002), 267-287, DOI: 10.1093/llc/17.3.267.

Friedrich Michael DIMPEL, Novellenschätze narratologisch auszeichnen und analysieren am Beispiel Victor von Scheffels ,Hugideo‘ und der sozialen Netzwerkanalyse, in: LiLi 47 (2017), 87-108, DOI:10.1007/s41244-0170044-8.

Maciej EdeR / Jan RYBICKI / Mike KeStemONT, stylo R package, 2017, online unter: https://sites.google.com/site/ computationalstylistics/stylo (26.06.2019).

Stephan Fuchs, Hybride Helden: Gwigalois und Willehalm. Beiträge zum Heldenbild und zur Poetik des Romans im frühen 13. Jahrhundert, Heidelberg 1997 (Frankfurter Beiträge zur Germanistik).

Algirdas Julien GrEIMAS, Strukturale Semantik. Methodologische Untersuchungen. Autorisierte Übersetzung aus dem Französischen von Jens Ihwe, Braunschweig [1966] 1971 (Wissenschaftstheorie, Wissenschaft und Philosophie 4).

Lena HetTINGer u. a., Genre classification on German novels, in: 26th International Workshop on Database and Expert Systems Applications (DEXA), 2015, 249-253.

Gert HüBNER, Erzählform im höfischen Roman. Studien zur Fokalisierung im ,Eneas', im ,Iwein' und im ,Tristan', Tübingen 2003 (Bibliotheca Germanica 44). 
Fotis JANNIDIS, Figur und Person. Beitrag zu einer historischen Narratologie, Berlin / New York 2004 (Narratologia 3).

Dorothea JANSEN, Einführung in die Netzwerkanalyse, Opladen 2003 (UTB 2241).

Benjamin KRAUTTER / Janis PAGEL, Klassifikation von Titelfiguren in deutschsprachigen Dramen und Evaluation am Beispiel von Lessings ,Emilia Galotti', in: Patrick Sahle, Hg., DHd 2019. Digital Humanities: multimedial \& multimodal. Konferenzabstracts, Köln 2019, 160-164.

Franco MORETTI, Distant Reading. Aus dem Englischen übersetzt von Christine Pries, Konstanz 2016 (2013).

Jan-Dirk MüLlER, Höfische Kompromisse. Acht Kapitel zur höfischen Epik um 1200, Tübingen 2007.

Thomas ProIsL u. a., Dependenzbasierte syntaktische Komplexitätsmaße, in: Patrick Sahle, Hg., DHd 2019. Digital Humanities: multimedial \& multimodal. Konferenzabstracts, Köln 2019, 270-273.

Christian SCHMID-CADALBERT, Der, OrtnitAW' als Brautwerbungsdichtung. Ein Beitrag zum Verständnis mittelhochdeutscher Schemaliteratur, Bern 1985 (Bibliotheca Germanica 28).

Armin Schulz, Poetik des Hybriden. Schema, Variation und intertextuelle Kombinatorik in der Minne- und Aventiureepik: ,Willehalm von Orlens ' - ,Partonopier und Meliur‘ - ,Wilhelm von Österreich - ,Die schöne MageIone', Berlin 2000 (Philologische Studien und Quellen 161).

Armin ScHULZ, Erzähltheorie in mediävistischer Perspektive. Herausgegeben von Manuel Braun / Alexandra Dunkel / Jan-Dirk Müller, Berlin / Boston 2012.

Mark TRAPpmAnN / Hans J. HummelL/ Wolfgang SodeUR, Strukturanalyse sozialer Netzwerke. Konzepte, Modelle, Methoden, Wiesbaden 2005.

Peer TRILCKE, Social Network Analysis (SNA) als Methode einer textempirischen Literaturwissenschaft, in: Philip Ajouri / Katja Mellmann / Christoph Rauen, Hg., Empirie in der Literaturwissenschaft, Münster 2013 (PoetogenesiStudien zur empirischen Anthropologie der Literatur 8), 201-247.

Michael VAUTH, Netzwerkanalyse narrativer Informationsvermittlung in Dramen, in: Patrick Sahle, Hg., DHd 2019. Digital Humanities: multimedial \& multimodal. Konferenzabstracts, Köln 2019, 184-188.

Rainer WARNING, Formen narrativer Identitätskonstitution im höfischen Roman, in: Odo Marquard / Karlheinz Stierle, Hg., Identität, München 1979 (Poetik und Hermeneutik 8), 553-589. 


\title{
historioPLUS
}

Fünf Jahre Studieren und digitales Publizieren in Salzburg

\section{Elias Knapp, Hester Margreiter \& Arno Strohmeyer}

Fachbereich Geschichte, Universität Salzburg

elias.knapp@sbg.ac.at

https://www.uni-salzburg.at/index.php?id=212090\&L=0

hester.margreiter@sbg.ac.at

https://www.uni-salzburg.at/index.php?id=204971\&L=0\&MP=200731-200747\%2C47-44788

arno.strohmeyer@sbg.ac.at

https://www.uni-salzburg.at/index.php?id=23315\&L=0\&MP=205208-207110

Keywords: historioPLUS, E-journal, Open Access

\begin{abstract}
Consistent promotion of young researchers is one of the general tenets of science management. In order to guarantee this, the Department of History at the University of Salzburg pursues a comprehensive approach with the e-journal historioPLUS.
\end{abstract}

\section{Vorbemerkungen}

Angesichts der stetigen technischen Neuerungen - Stichwort ,Digitalisierung - haben sich auch die Arbeitsweisen, Methoden und Fragestellungen bzw. Forschungsgegenstände in den Geisteswissenschaften tiefgreifend verändert. Damit gehen digitale Publikationsformen einher, die der Wissenschaft neue und vermehrte Möglichkeiten bieten, die (Fach-)Öffentlichkeit zu erreichen. ${ }^{1}$ In dieser Entwicklung spielt das Konzept des Open Access - des kostenfreien Zuganges zu wissenschaftlichen Forschungsergebnissen - eine maßgebliche Rolle. ${ }^{2}$ Das zeigen nicht zuletzt die diesbezüglich angepassten Richtlinien des österreichischen Fonds zur Förderung der wissenschaftlichen Forschung (FWF), die die Mitarbeiter*innen aller nach dem 1. Jänner 2019 bewilligten Projekte verpflichten, ihre im Rahmen der Vorhaben erstellten „referierten Publikationen“ und „Forschungsdaten“ digital und kostenfrei zugänglich zu machen. ${ }^{3}$

Dieser Beitrag stellt historioPLUS, das „E-Journal für Arbeiten von Studierenden des Fachbereichs Geschichte der Universität Salzburg“ (www.historioplus.at), vor, das Studierenden die Möglichkeit

1 Vgl. einführend Hubertus KoHLE, Digitales Publizieren, in: Fotis Jannidis / Hubertus Kohle / Malte Rehbein, Hg., Digital Humanities. Eine Einführung, Stuttgart 2017, 199-205. Ein aktuelles Beispiel bietet das Portal MEMO, das vor allem vom Institut für Realienkunde des Mittelalters und der frühen Neuzeit (Universität Salzburg) getragen wird. Siehe: Medieval and Early Modern Material Culture Online (MEMO), online unter: http://memo.imareal.sbg.ac.at/ (02.04.2019).

2 Siehe dazu u. a. Konstanze Söllner / Bernhard MitTERMAIER, Hg., Praxishandbuch Open Access, Berlin 2017.

3 Vgl. FWF, Open-Access-Policy, online unter: https://www.fwf.ac.at/de/forschungsfoerderung/open-access-policy/ (02.04.2019). 
eröffnet, ihre Arbeiten zu publizieren. Themen sind vor allem das Schaffen der entsprechenden Infrastruktur, die Ziele, die mit dem Projekt verfolgt werden, die erwünschten Mehrwerte sowie die redaktionellen Arbeitsweisen. ${ }^{4}$

\section{Von der Idee zur Umsetzung}

Im Juni 2014 wurden die ersten fünf Beiträge von historioPLUS veröffentlicht. Die Idee, einige Arbeiten von Studierenden über eine eigene Onlineplattform einer breiten Öffentlichkeit zugänglich zu machen, reicht allerdings weiter zurück. Die Ursprünge von historioPLUS liegen im Frühjahr 2012 und gehen auf Initiativen unterschiedlicher Mitglieder des Fachbereichs Geschichte zurück, die sich zu dieser Zeit in sehr verschiedenen Positionen und Karrierephasen befanden. Auch von Seite der Studierenden - insbesondere der Studienrichtungsvertretung - kamen Denkanstöße. Die Leitung des Projekts übernahm von Beginn an Arno Strohmeyer (Univ.-Prof. für Allgemeine Geschichte der Neuzeit). Im Sommer 2012 wurde eine Arbeitsgruppe geschaffen, um konkrete Überlegungen für die Umsetzung zu sammeln. Es stellten sich u. a. Fragen hinsichtlich des Publikationsformats der Texte und der Vorgaben für die Zitierweise, aber auch bezüglich der Kriterien für die Einreichung und Begutachtung der Beiträge. Anregungen holte sich die Arbeitsgruppe von ähnlichen, bereits laufenden Projekten innerhalb Österreichs, darunter das Open-Access-Journal des Fachbereichs Kommunikationswissenschaft der Universität Salzburg, kommunikation.medien ${ }^{5}$, und das Projekt des Instituts für Geschichtswissenschaften und Europäische Ethnologie der Universität Innsbruck, historia.scribere ${ }^{6}$.

Die Frage des Namens und des Logos für die Publikationsplattform stellten eine spezielle Herausforderung dar, da diese hinsichtlich Außenwirkung von besonderer Bedeutung sind. Schließlich sollte dadurch ein Wiedererkennungswert geschaffen und eine Identifikation mit dem Fachbereich Geschichte der Universität Salzburg erreicht werden. Nach einer Umfrage unter den Studierenden standen u. a. Titel wie historia_nostra, publi_online, SafeHistory und StudiPuPS (für Studierendenpublikationsplattform Salzburg) im Raum. Letztlich fiel die Wahl auf den Namen historioPLUS - „historio“ steht für den Begriff „Geschichte“ in der Plansprache Esperanto und „PLUS“ stellt die gängige Abkürzung für die „Paris Lodron Universität Salzburg“ dar.

\section{Ziele bzw. Mehrwert: historioPLUS als Ausbildungs- und Repräsentationsmedium}

Welche konkreten Ziele werden mit dem Projekt historioPLUS verfolgt? Kurz formuliert dient das E-Journal als Ausbildungs- und Repräsentationsmedium. Publiziert werden hervorragende Arbeiten von Studierenden. Begutachtet, ausgewählt und redaktionell betreut werden diese von einem Redaktionsteam bestehend aus Professor*innen, Post-Docs, Doktorand*innen und Studierenden sowie Fachbereichsmitgliedern aus dem allgemeinen Universitätspersonal. Daraus ergeben sich einige Ziele bzw. Mehrwerte:

4 Einen kürzeren Überblick zu Entstehung und Umsetzung von historioPLUS gibt ein Blogbeitrag von Susanne Höll, der anlässlich des Workshops „e-Journals - Geschichte, Kulturwissenschaft und Archivwesen im Open Access“ im Jahr 2016 entstanden ist. Vgl. Susanne HöLL, historioPLUS - E-Journal für Arbeiten von Studierenden des Fachbereichs Geschichte der Universität Salzburg, online unter: https://bioeg.hypotheses.org/1704 (02.04.2019).

5 Vgl. kommunikation.medien. Open-Access-Journal für den wissenschaftlichen Nachwuchs, online unter: http://digital. obvsg.at/obvusbjkm (02.04.2019).

6 Vgl. historia.scribere. Online-Zeitschrift der Institute für Alte Geschichte und Altorientalistik, Geschichtswissenschaften und Europäische Ethnologie sowie Zeitgeschichte der Universität Innsbruck zur Publikation studentischer Arbeiten, online unter: http://historia.scribere.at/ (02.04.2019). 
- Ein bedeutendes Ziel ist das Sichtbarmachen einzelner Forschungsleistungen von Studierenden, die ansonsten häufig in der sprichwörtlichen Schublade ,verstauben'. Mit der Aussicht auf eine Publikationsmöglichkeit sollen die Studierenden zudem motiviert werden, eigenständig quellengestützte Forschung zu betreiben - das gilt insbesondere für jene, die eine wissenschaftliche Laufbahn anstreben.

- Die Beiträge erfüllen eine Vorbildwirkung für das Verfassen wissenschaftlicher Arbeiten und sollen speziell den Neuzugängen unter den Studierenden eine Hilfestellung liefern.

- Mit der Einführung und andauernden Aktualisierung der inzwischen sehr ausführlichen Zitierrichtlinien und der Vorschläge zur Textgestaltung, die online zugänglich sind, wird die Lehre diesbezüglich erleichtert.

- Die Möglichkeit eines Engagements im Redaktionsteam erlaubt es Studierenden und jungen wissenschaftlichen Mitarbeiter*innen, Einblicke in redaktionelle Tätigkeiten zu erhalten. Dabei können diese Erfahrungen darin sammeln, Texte kritisch zu besprechen, die eigene Ansicht gegenüber anderen zu artikulieren und die Herangehensweise von fortgeschrittenen Forschenden kennenlernen. Damit dient historioPLUS zur Ausbildung und Förderung des wissenschaftlichen Nachwuchses.

- Schlussendlich veranschaulicht die Themenvielfalt der veröffentlichten Arbeiten die inhaltliche Breite in Forschung und Lehre am Fachbereich Geschichte der Universität Salzburg, kommuniziert diese nach außen und trägt damit dazu bei, den Fachbereich in der wissenschaftlichen Community zu positionieren.

\section{Arbeitsprozess: Redaktion - Auswahlverfahren - Veröffentlichung}

Bei historioPLUS werden ausschließlich Aufsätze mit einer Länge von etwa 85.000 Zeichen (also ca. 30 Seiten) veröffentlicht. Eingereicht werden können sowohl Proseminar- und Seminararbeiten als auch umfangreichere Qualifikationsarbeiten (Bachelor-, Diplom- und Masterarbeiten). Bei Letzteren wird im Fall einer Annahme durch die Redaktion der/die Autor*in aufgefordert, einen auf der Qualifikationsarbeit basierenden und dem Format entsprechenden Aufsatz zu verfassen. Für eine Publikation im jeweils aktuellen Jahrgang kommen nur jene Arbeiten infrage, die im Rahmen von Lehrveranstaltungen im vorangegangenen Studienjahr entstanden sind. Deadline für die Einreichung ist immer der 15. Dezember. ${ }^{7}$

Wie oben bereits erwähnt, setzt sich das Redaktionsteam aus Fachbereichsmitgliedern unterschiedlichster Karrierestufen und inhaltlicher Spezialisierung sowie Studierenden zusammen; die Begutachtung erfolgt also durch Personen aus allen wissenschaftlichen Ebenen. Derzeit umfasst das Redaktionsteam, das sich in der Regel pro Studienjahr vier bis fünf Mal trifft, 16 Personen. Angesichts der Vielzahl an befristeten Vertragsverhältnissen, insbesondere im wissenschaftlichen Mittelbau, stellt die Kontinuität unter den Mitarbeitenden ein besonderes Problem hinsichtlich der Erhaltung und (Weiter-) Entwicklung des E-Journals dar. Das Redaktionsteam ist dementsprechend einem steten personellen Wandel unterworfen, mit dem auch die Schwierigkeit der Qualitätssicherung einhergeht.

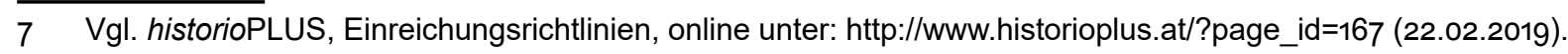


Um den Erhalt der Qualität und ein systematisches Auswahlverfahren zu gewährleisten, wurde für die Bewertung der eingereichten Arbeiten ein Peer-Review-Schema erstellt. Dieses berücksichtigt u. a. Aspekte wie:8

- Die Originalität und wissenschaftliche Aktualität des Themas bzw. der Fragestellung,

- den Bezug zur Fachwissenschaft,

- die wissenschaftliche Erschließung,

- das Methodenbewusstsein,

- die Struktur und den Aufbau,

- das sprachliche Niveau

- und eine korrekte Zitierweise.

Wurde eine Arbeit zur Veröffentlichung angenommen, wird diese von dem/der Autor*in, unterstützt durch den/die Betreuer*in und in Kooperation mit dem Kernteam der Redaktion, für die Publikation vorbereitet. Letzteres bestehend aus Arno Strohmeyer (Herausgeber), dem/der Post-Doc-Mitarbeiter*in im Bereich Neuere Geschichte (aktuell Lena Oetzel), dem/der Leiter*in des Redaktionsbüros (Mitarbeiter*in allg. Universitätspersonal Neuere Geschichte; aktuell Birgit Brenner) und dem/der Studienassistent*in (aktuell Elias Knapp). Die Koordination der Einreichungen, des Begutachtungsprozesses sowie der Endredaktion und Veröffentlichung der Beiträge übernimmt der/die Leiter/in des Redaktionsbüros.

Veröffentlicht werden die meist fünf bis sechs Beiträge als einzelne PDF-Dateien einmal jährlich, am Ende des Sommersemesters, auf der Website von historioPLUS. Der 6. Jahrgang (2019) ist in der letzten Juni-Woche online gegangen. Inhaltlich erschlossen sind die einzelnen Aufsätze mittels kurzer Abstracts, die einen Einblick zu den Beiträgen liefern. Darüber hinaus sind alle Texte mit ca. vier bis fünf Schlagwörtern verknüpft, die in einer Sidebar auf der Website aufscheinen und zu thematisch passenden Aufsätzen führen.

\section{Neuerungen und Perspektiven}

Das Kernteam der Redaktion ist laufend daran interessiert, die Inhalte des Internetauftritts und das E-Journal im Allgemeinen zu aktualisieren und weiterzuentwickeln: So wurden bspw. die Kriterien der Textauswahl im November 2018 öffentlich gemacht und seit Juni 2019 sind die Aufsätze auch über gängige externe Netzwerke (Verbund- und Bibliothekskataloge etc.) sowie den Publikationsserver der Universität Salzburg, ePLUS, abrufbar, was deren öffentliche Sichtbarkeit deutlich erhöht.

Ein verstärkter Ausbau von historioPLUS zur Lehrplattform steht ebenfalls im Raum. Derzeit wird an einer Aktualisierung der Richtlinien zur Textgestaltung sowie einer Word-Vorlage für die vorbildliche Formatierung gearbeitet, die demnächst zugänglich gemacht werden.

Andere potenzielle Entwicklungsoptionen sind die Prämierung von Arbeiten, wie es bei der Innsbrucker Plattform historia scribere üblich ist, eine Ausweitung auf weitere Texttypen, z.B. Exkursionsberichte, oder eine stärkere Öffnung hin zu anderen Fächern bzw. Fachbereichen.

8 Siehe umfangreicher: historioPLUS, Auswahlkriterien, online unter: http://www.historioplus.at/?page_id=1057 (22.02.2019). 


\section{Literaturverzeichnis}

FWF, Open-Access-Policy, online unter: https://www.fwf.ac.at/de/forschungsfoerderung/open-access-policy/ (02.04.2019).

historia.scribere. Online-Zeitschrift der Institute für Alte Geschichte und Altorientalistik, Geschichtswissenschaften und Europäische Ethnologie sowie Zeitgeschichte der Universität Innsbruck, online unter: http://historia. scribere.at/ (02.04.2019).

historioPLUS. E-Journal für Arbeiten von Studierenden des Fachbereichs Geschichte der Universität Salzburg, online unter: http://www.historioplus.at/ (25.04.2019).

Susanne HöLL, historioPLUS - E-Journal für Arbeiten von Studierenden des Fachbereichs Geschichte der Universität Salzburg, online unter: https://bioeg.hypotheses.org/1704 (02.04.2019).

Hubertus KoHLE, Digitales Publizieren, in: Fotis Jannidis / Hubertus Kohle / Malte Rehbein, Hg., Digital Humanities. Eine Einführung, Stuttgart 2017, 199-205.

kommunikation.medien. Open-Access-Journal für den wissenschaftlichen Nachwuchs, online unter: http://digital. obvsg.at/obvusbjkm (02.04.2019).

Medieval and Early Modern Material Culture Online (MEMO), online unter: http://memo.imareal.sbg.ac.at/ (02.04.2019).

Konstanze Söllner / Bernhard MitTermaier, Hg., Praxishandbuch Open Access, Berlin 2017. 


\title{
Marketing trifft Forschung
}

\author{
Zielgruppenorientierte Digitalisierung Wissenschaftlicher Inhalte
}

\section{Johannes Ebner [1], Peter Färberböck [1] \& Stefan Schwaiger [2]}

[1] FB Geschichte, Universität Salzburg

johannes.ebner@marketext.at

peter.faerberboeck@marketext.at, https://orcid.org/0000-0002-8701-8259

[2] FB Germanistik, Universität Salzburg

stefan.schwaiger@marketext.at

www.marketext.at

Keywords: Content Marketing, Science Marketing, Audience-Oriented Digitalization

\begin{abstract}
Since the Bologna Process, the competition in higher education grew harsher. Thus, a target group oriented marketing approach in websites is desperately needed. This article depicts the approach of the Department of History of the University of Salzburg.
\end{abstract}

\section{Einleitung}

Im Zuge dieser Arbeit können wir nicht auf alle Gegebenheiten, wie Suchmaschinenoptimierung (SEO), Usability (Stichwort User Experience / UX) und Konkurrenzanalyse eingehen, da dies den Rahmen deutlich sprengen würde. Deswegen wird ein Überblick über die Projektarbeit der Website des Fachbereichs Geschichte gegeben und insbesondere auf am Anfang unvorhersehbare Herausforderungen und Besonderheiten eingegangen.

Konkret überarbeiten wir die Website des Fachbereichs Geschichte der Universität Salzburg. Dabei kam es zu einigen Herausforderungen, die von vornherein nicht offensichtlich waren. Im nächsten Kapitel werden einige dieser Herausforderungen vorgestellt und anschließend die ergriffenen Maßnahmen beschrieben. Am Beginn fokussieren wir uns jedoch auf die Gründe für zielgruppenorientierte Digitalisierung.

\section{Die Ausgangslage: Warum eigentlich Wissenschaftsmarketing?}

Institutionen haben gegenwärtig ein Problem, das gleichzeitig eine Chance darstellt: Sie können wissenschaftliche Inhalte und Erkenntnisse vermarkten oder nicht. Hier wiederum taucht ein „Warum“ auf. Warum sollte man wissenschaftliche Desiderata und Erkenntnisse mit Content-Marketing-Maßnahmen präsentieren? Eine kurze Erklärung: Content-Marketing vermittelt „Inhalte, die informierend, beratend und unterhaltend sind“" , und bietet so einen Mehrwert für Kund*innen, in diesem Fall angehende Stu-

1 Lutz FRÜHBRODT, Content Marketing. Wie „Unternehmensjournalisten“ die öffentliche Meinung beeinflussen, Frankfurt am Main 2016, 7, online unter: https://www.otto-brenner-stiftung.de/fileadmin/user_data/stiftung/o2_Wissenschaftsportal/o3_ Publikationen/AH86_Contentmarketing_Fruehbrodt_2016_06_09.pdf (04.04.2019). 
dierende oder wissenschaftlichen Nachwuchs. Eine Antwort auf dieses „Warum“ liegt hier in der Vereinheitlichung durch den Bologna-Prozess ${ }^{2}$, durch den neue Ausbildungsinstitutionen entstanden sind und sich gleichzeitig der wissenschaftliche Nachwuchs verringert.

Der Wettbewerb um die Bildung ist in vollem Gange. Dieser Wettbewerb erfordert eine Legitimation der Institutionen und Fachbereiche. Um Legitimation zu erlangen, braucht es eine Informationsoffensive.

Wettbewerb erfordert Legitimation.

Legitimation erfordert Information.

In der Studie create your UNIverse ${ }^{3}$ wurden Studierende befragt, was sie von Hochschulen und Forscher*innen erwarten. Aus insgesamt 2.105 Beiträgen (auch in sozialen Medien) von 754 Teilnehmer*innen folgerten sie fünf Handlungsempfehlungen für die künftige Gestaltung der Hochschule: ${ }^{4}$

- Digitalisierung besser nutzen

»Proaktiv und strategisch sowie virtuell und physisch

- Weiterentwicklung der Lehrenden

»Entwicklung von reinen Wissensvermittler*innen zu Lerncoaches und Mentor*innen

- Bildung von Strukturen, die Vernetzung und Lifelong-Learning ermöglichen

»Vernetzung von Studierenden, Alumni, Lehrenden, aber auch Unternehmen, NGOs und dem öffentlichen Sektor

- Alternative Lehrveranstaltungen

» Experimentierräume für alle Studierenden in der Lehre, die Interaktivität fördern. Gemeinsam forschen

- Ausbau internationaler und nationaler Zusammenarbeit

»Digital und nicht-digital

Diese Empfehlungen sind nur ein Ausblick in die Zukunft, was möglich wäre und wohin es, zumindest aus Sicht der Studienautor*innen, gehen soll. Dazu braucht es hingegen von der Seite der Universität, des Instituts oder des Fachbereichs einen stimmigen Markenauftritt, der Besucher*innen überzeugen soll. Ein Ausschnitt dieses Markenauftritts ist die Kommunikation nach außen und damit die inhaltliche Aufbereitung - das Content-Marketing - der Website.

Kurz: Marketing ist nötig, wo eine Wettbewerbssituation herrscht. Darunter fällt auch das angesprochene Content-Marketing.

Um zurück zum zentralen Punkt der Legitimation zu kommen, gilt es, diese Hinweise als Lösungsansätze im Rahmen der angesprochenen Legitimation zu sehen. Durch die aufbereiteten Inhalte, die

2 N.N., European Higher Education Area and Bologna Process, online unter: http://www.ehea.info/ (04.04.2019) und ausführlicher in Lena BECKER, Bildung im Zeichen der Ökonomisierung. Der Bologna-Prozess und seine Auswirkungen auf die Erziehungswissenschaft, Darmstadt 2012.

3 Im Auftrag der Ludwig Boltzmann Gesellschaft und des austrian council, durchgeführt durch das Consulting-Unternehmen winnovation consulting gmbh.

4 Vgl. Gertraud LeIMÜLLER u. a., create your UNIverse. Erwartungshaltungen Studierender an die Hochschulen der Zukunft, Wien 2017, 33-35, online unter: https://www.rat-fte.at/files/rat-fte-pdf/publikationen/2017/170427_Create\%20your\%20 UNIverse_finale\%20Praesentation.pdf (04.04.2019). 
mit diesem Ziel im Fokus entstanden sind und weiterhin entstehen (Inhalte bleiben nicht für immer stehen, sondern werden stets angepasst), sollen sich die ausgewählten Zielgruppen angesprochen fühlen. Sie werden dadurch ermuntert, sich individuell mit diesen auseinanderzusetzen.

\section{Mannigfaltige Herausforderungen - Von Stakeholdern bis zu Bildrechten}

Eines der Grundprobleme aller größeren Projekte - auch eine Neuaufstellung einer Fachbereichswebsite ist als großes Projekt zu sehen und war auch als solches geplant - ist die Absprache mit den Stakeholdern. Allgemein ist ein Stakeholder eine Gruppe oder eine Person, die ein berechtigtes Interesse am Verlauf oder Ergebnis eines Prozesses oder Projektes hat. ${ }^{5}$ Dabei ist nicht nur das umgangssprachliche ,mit ins Boot holen' gemeint, sondern auch die genaue Abstimmung und transparente Kommunikation mit allen Beteiligten. Hier fällt auch die zeitliche Terminierung ins Gewicht, denn je mehr Stakeholder ein Projekt hat, desto komplexer wird es, Lösungsansätze in den Tagesablauf einzubinden. Hier kann es zu Zeitverzögerungen kommen - vor allem bei der Terminierung von größeren Meetings oder der Absprache mit mehreren Stakeholdern.

Im Fall der Website des Fachbereichs Geschichte wurde dies über eine Arbeitsgruppe Website (aus ursprünglich acht Personen) abgewickelt, die sich regelmäßig traf. Hier informierten wir die Gruppe über den Projektstand und konnten unmittelbar Feedback einholen und entsprechend nachkorrigieren. Der Fachbereichsrat, der aus dem Vorsitzenden, Vertreter*innen der Professor*innen, des akademischen Mittelbaus, der Studierenden, des allgemeinen Universitätspersonals, der Personalvertretung und dem Arbeitskreis für Gleichbehandlungsfragen besteht, diente als Lenkungsausschuss. ${ }^{6}$

Eines der prägenden Stichwörter der Organisation war die verteilte Verantwortlichkeit. Texte der Website, die eigentlich auf derselben Informationsebene existierten, z. B. Beschreibungen von zwei Forschungssäulen ${ }^{7}$ oder Kernfächern, hatten verschiedene Verantwortliche. Dies ist kein Novum, sondern bei der ,natürlichen“ Entstehungsgeschichte einer Seite sehr oft die Norm. Durch die verteilte Verantwortlichkeit waren die Texte jedoch uneinheitlich, weil die Absprache untereinander fehlte. Durch die fließende Entstehung der Inhalte gab es Unterschiede in der Informationsdichte, aber auch größere Abweichungen in Länge, Aufbau und auch Gliederung. Diese größere Inkohärenz ist bei einem Webauftritt, der möglichst konsistent und stringent wirken sollte, ein größeres Problem und vermittelt unfreiwillig Unprofessionalität.

Zusätzlich zu den internen Abstimmungsaufgaben des Projekts war der allgemeine Aufbau der Website ein Arbeitspaket. Die Fachbereichsseite unterliegt der Corporate Identity der Website der Universität Salzburg und somit auch dem allgemeinen Aufbau dieser. Dies äußert sich dadurch, dass der Aufbau und auch der Inhalt einer Seite nicht komplett vom Fachbereich bestimmt werden kann. Im Gegenteil: Nur der mittlere Bereich kann befüllt werden, die außenliegenden Bilder, News-Spalten oder das Menü sind starr vorgegeben. ${ }^{8}$

5 Sonja EILMANN u. a., Interessengruppen / Interessierte Parteien, in: Michael Gessler, Hg., Kompetenzbasiertes Projektmanagement. Band 1, Nürnberg 2011, 71.

6 Vgl. N. N., Fachbereichsrat des Fachbereichs Geschichte, online unter: https://uni-salzburg.at/index.php?id=23305 (04.04.2019).

7 Forschungssäulen sind Forschungsgruppen des Fachbereichs, auf die sich die einzelnen Forschenden in Gruppen spezialisiert haben, vgl. N. N., Forschungssäulen. Fachbereich Geschichte, online unter: https://uni-salzburg.at/index. php?id=202971 (04.04.2019).

8 Vgl. N. N., Kernfächer und Arbeitsbereiche. Fachbereich Geschichte, online unter: https://uni-salzburg.at/index. php?id=23385 (04.04.2019). Hier kann nur der mittlere Text geändert werden und höchstens mit Bildern angereichert 


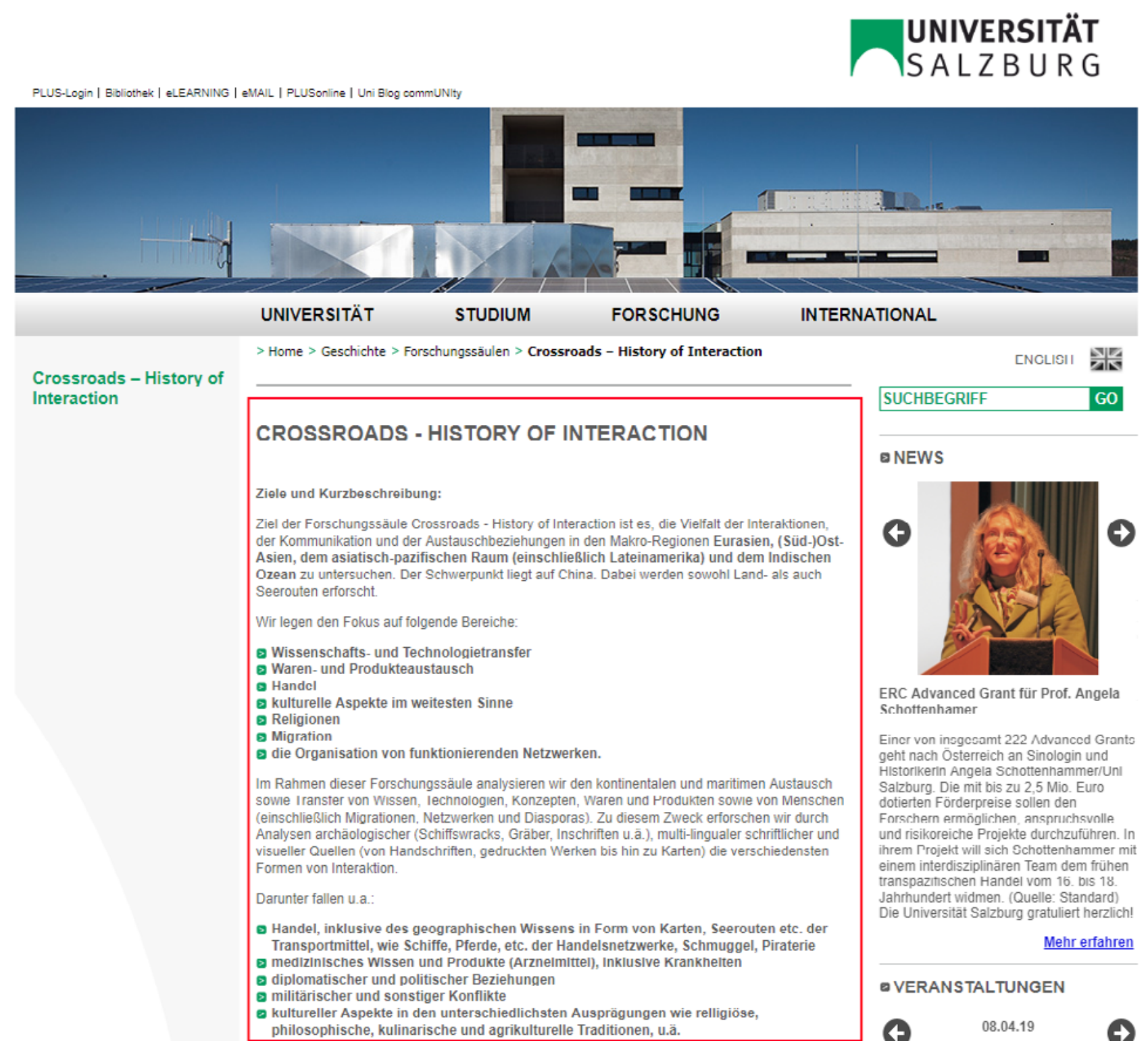

Abb. 1: Eigener Screenshot, Website des Fachbereichs Geschichte. Der mittlere Teil, der befüllt werden kann, ist rot markiert.

Die Verantwortung der einzelnen Websites oblag dem wissenschaftlichen Personal, was zu einem weiteren Problem führte: Die Sprache im Content-Marketing ist einfacher, schnell verständlich und auch mit weniger Aufmerksamkeit lesbar. Dazu soll sie begeistern. Die vorher genutzte wissenschaftliche Sprache war hingegen deutlich komplexer. Unsere Aufgabe war es, diese zwei Welten zu vereinen: Die ausgewiesene Fachkunde weiterhin im Vordergrund zu behalten und gleichzeitig attraktiv die Zielgruppen zu bedienen. Hier war eine Herausforderung, die Verantwortlichen davon zu überzeugen, dass kurze, einfache Texte mit stärkerer Bebilderung das probate Mittel für die Fachbereichswebsite sind. Der PR-Effekt der Seite musste erst ins Bewusstsein gerufen werden. Die Inhalte wären nämlich öffentlichkeitswirksam, einzig die Präsentation musste angepasst werden. In diesem Zuge ist auch die Definition der Zielgruppe unumgänglich - die dazu definierten Personas sind im nächsten Kapitel beschrieben.

und verschönert werden. Der Bereich rundherum ist vorgegeben. 
Am Rande sind auch die Bildrechte eine Herausforderung. Verwendete Bilder und Videos werden deswegen zum Großteil vom Fachbereich selbst gemacht. Von den Beteiligten werden die Bildrechte schriftlich eingeholt. Bei den Stock-Fotos ${ }^{9}$ müssen diese gegebenenfalls gekauft werden, sofern sie nicht frei verwendbar sind.

\section{Planung, Zielfindung und Umsetzung der zielgruppenorientierten Digitalisierung}

In den folgenden Absätzen wird auszugsweise beschrieben, welche Grundmaßnahmen angewandt wurden, um so zur gewünschten zielgruppenorientierten Digitalisierung zu kommen. Wie angesprochen, war der Grundstein für die Planung die Gründung einer Task Force, die mit Schlüsselpersonen (Fachbereichsleitung, IT, Sekretariat) besetzt wurde. In dieser wurde die Planung und die Umsetzung des Projekts besprochen. Zusätzlich kam diese zu Workshops zusammen, wo grundlegende Richtungsentscheidungen festgelegt und z. B. Stakeholder, Personas und Konkurrenz zumindest grob identifiziert wurden.

Einer der nächsten Schritte war ein Content-Audit der Seite. Dieser stellt eine Art Prüfung aller derzeit verfügbaren Web-Inhalte einer Seite dar. ${ }^{10}$ Dabei wird die Website qualitativ ausgewertet und nach Verbesserungsbedarf gesucht. Jede einzelne Seite wurde kategorisiert (About, News, etc.), die potenzielle Zielgruppe der Seite identifiziert und die verwendeten Inhaltstypen aufgelistet. Aufgrund dessen wurde der Inhalt nach Qualität bewertet sowie positive und negative Merkmale hervorgehoben, sodass diese in die zukünftige Seite übernommen bzw. verhindert werden können.

Ein weiterer Kernschritt war die Erstellung von Personas. Diese sind fiktionale Figuren, die jeweils einen spezifischen Typ eines potenziellen Benutzers charakterisieren. ${ }^{11}$ Dazu werden grobe soziodemografische Daten der jeweiligen Zielgruppensegmente nötig, aber auch grobe Umschreibungen der Gefühlswelt, wie Ängste, die Lebenswelt und die Motivation, die die Person antreibt, eingearbeitet. Selbst ein simulierter ,Kaufprozess', d. h. wie würde er oder sie sich auf der Website fortbewegen und was sind seine Zielseiten, sowie die vermutete Mediennutzung (z. B. soziale Medien, Offline-Medien oder Handy-Nutzung) werden implementiert. Durch diesen genauen Steckbrief können Inhalte speziell für die Zielgruppensegmente erstellt werden.

Die deutlichen Einschränkungen durch die fixe Vorgabe der Universitäts-Website führte zu Kompromissen und auch anderen Ideen. Eine davon ist eine eigene Seite für Studierende in Blog-Form basierend auf WordPress, die optisch und inhaltlich schneller und einfacher angepasst werden kann. Diese Flexibilität ermöglicht es erst, optisch und technisch dem schnelllebigen Zeitgeist des Internets nachzukommen. Diese Idee wird in naher Zukunft umgesetzt und befindet sich in der Endphase der Planung und Vorbereitung. Andere Bereiche bekommen zielgruppengerechte Texte und werden stärker bebildert. Auch an Video-Inhalten wird derzeit gearbeitet - diese befinden sich noch in der Konzeptphase.

$9 \quad$ Stock Fotos sind in Datenbanken erhältlich und je nach Benutzung unterliegen sie verschiedenen Lizenzen. Sie können kostenlos oder gebührenpflichtig sein. Vgl. Michael R. PERES, The Focal Encyclopedia of Photography. Digital Imaging, Theory and Applications, History and Science, New York / London 2007, 351.

10 Vgl. Kristina HALVORSON / Melissa RACH, Content Strategy for the Web, Berkeley 2012, 46-67. Unsere Vorgehensweise wird grob in folgendem Blogpost skizziert: Johannes EBNER / Peter FÄRBERBÖcK / Stefan ScHWAIGER, Die Definition des Content Audits, online unter: https://www.marketext.at/blog/definition-des-content-audits/ (04.04.2019).

11 Vgl. Jill ButLER / Kritina HoLDEN / William LIDWELL, Universal Principles of Design. A Cross-disciplinary Reference, Beverly 2010, 182. 


\section{Fazit}

»Eine Website ist doch schnell gemacht. Die Texte schreiben wir selbst - als Historiker*innen ist das unser tägliches Brot«. Genau so könnte man sich die Ausgangslage vorstellen, wenn eine Fachbereichsseite erstellt wird. Diese Annahme ist nicht völlig falsch, denn tatsächlich ist die Verschriftlichung ein großer Teil des Historikerberufes. Dabei werden aber einige Dinge übersehen, denn auch wissenschaftliche Papers haben eine definierte Zielgruppe, für die man schreibt. So funktioniert es auch bei Web-Texten. Dieses Projekt stellt dabei den Grundplan für einen sich stetig ändernden Prozess dar. Die Task Force, die Personas und die Veränderung der technischen Möglichkeiten beeinflussen diesen stets. Es steht am Ende kein fertiger digitaler Auftritt, der über Jahre gültig ist, sondern vielmehr ein Gerüst, das stets gepflegt und angepasst werden muss. Ein solcher Prozess ist inhärent nötig, um in der heutigen Konkurrenzsituation als wissenschaftliche Institution zu überleben.

\section{Literaturverzeichnis}

Lena BECKER, Bildung im Zeichen der Ökonomisierung. Der Bologna-Prozess und seine Auswirkungen auf die Erziehungswissenschaft, Darmstadt 2012.

Jill BUTLER / Kritina HoLden / William LIDWELL, Universal Principles of Design. A Cross-disciplinary Reference, Beverly 2010.

Johannes EBNER / Peter FÄRBERBÖCK / Stefan SchWAIGER, Die Definition des Content Audits, online unter: https:// www.marketext.at/blog/definition-des-content-audits/ (04.04.2019).

Sonja EILMANN u. a., Interessengruppen / Interessierte Parteien, in: Michael Gessler, Hg., Kompetenzbasiertes Projektmanagement. Band 1, Nürnberg 2011.

Lutz FRÜHBRODT, Content Marketing. Wie „Unternehmensjournalisten“ die öffentliche Meinung beeinflussen, Frankfurt am Main 2016, online unter: https://www.otto-brenner-stiftung.de/fileadmin/user_data/stiftung/02_ Wissenschaftsportal/03_Publikationen/AH86_Contentmarketing_Fruehbrodt_2016_06_09.pdf (04.04.2019).

Kristina HALVORSON / Melissa RaCH, Content Strategy for the Web, Berkeley 2012.

Gertraud LEIMÜLLER U. a., create your UNIverse. Erwartungshaltungen Studierender an die Hochschulen der Zukunft, Wien 2017, online unter: https://www.rat-fte.at/files/rat-fte-pdf/publikationen/2017/170427_Create\%20 your\%20UNIverse_finale\%20Praesentation.pdf (04.04.2019).

N. N., European Higher Education Area and Bologna Process, online unter: http://www.ehea.info/ (04.04.2019).

N. N., Fachbereichsrat des Fachbereichs Geschichte, online unter: https://uni-salzburg.at/index.php?id=23305 (04.04.2019).

N. N., Forschungssäulen. Fachbereich Geschichte, online unter: https://uni-salzburg.at/index.php?id=202971 (04.04.2019).

N. N., Kernfächer und Arbeitsbereiche. Fachbereich Geschichte, online unter: https://uni-salzburg.at/index.php?id=23385 (04.04.2019).

Michael R. PERES, The Focal Encyclopedia of Photography. Digital Imaging, Theory and Applications, History and Science, New York / London 2007. 


\title{
Der Nachlass als Netzwerk
}

\author{
Zur Entwicklung einer Nachlass-Ontologie am Beispiel des Projekts \\ „Stefan Zweig digital“
}

\section{Christopher Pollin [1] \& Lina Maria Zangerl [2]}

[1] Universität Graz, Zentrum für Informationsmodellierung - Austrian Centre for Digital Humanities christopher.pollin@uni-graz.at

[2] Universität Salzburg, Literaturarchiv Salzburg - Forschungszentrum von Universität, Land und Stadt Salzburg

linamaria.zangerl@sbg.ac.at, https://orcid.org/0000-0001-9709-3669

Keywords: Semantic Web, Nachlass, Ontology, Stefan Zweig digital, Resource Discovery, Text Encoding Initiative (TEI), Austrian Literature

\begin{abstract}
Within the project "Stefan Zweig digital", a digital reconstruction of the posthumous papers of Stefan Zweig, a domain-specific ontology is modeled to represent context-sensitive cultural heritage objects flexibly and expressively according to the requirements of researchers.
\end{abstract}

\section{Einleitung}

Kulturerbeobjekte und ihre Metadaten - als geisteswissenschaftliche Daten verstanden - bringen besondere Herausforderungen mit sich: Sie sind kontextabhängig, haben bestimmte (abstrakte) Beziehungen zu anderen Objekten und weisen interne und externe Verknüpfungen zu Personen, Orten, Werken und (biographischen) Ereignissen, sowie Sekundärquellen auf. Im Hinblick auf Nachlässe ergibt sich zudem die Notwendigkeit einer Darstellung des Gesamtzusammenhangs des Materials, das sich in Unikalität, Umfang, Informalität und Individualität von klassischem Archivgut unterscheidet. ${ }^{1}$ Ein Repository zur Langzeitarchivierung und Semantic-Web-Technologien können dabei helfen, diesen Herausforderungen entgegenzutreten.

Im Rahmen des Projekts „Stefan Zweig digital“ (SZD), ${ }^{2}$ einer digitalen Rekonstruktion des Nachlasses von Stefan Zweig, wird eine domänenspezifische Nachlass-Ontologie modelliert. Ziel einer solchen Nachlass-Ontologie ist es, auf konzeptioneller Ebene semantische Interoperabilität, Nachvollziehbarkeit und Dokumentation zu gewährleisten, aber auch kontextsensitive Nachlassobjekte flexibel und ausdrucksstark nach den Anforderungen der Fachwissenschaftler*innen abbilden zu können.

1 Dirk WEISBROD, Langzeitarchivierung digitaler Schriftstellernachlässe. Eine Bestandsaufnahme in den Literaturarchiven Deutschlands, Österreichs und der Schweiz, in: Young Information Scientist 2 (2017), 1-12, hier 10, online unter: http:// eprints.rclis.org/30976/ (12.06.2019) www.stefanzweig.digital. 


\section{Das Projekt Stefan Zweig digital}

Im Zentrum der Forschungs- und Informationsplattform www.stefanzweig.digital, die seit Juni 2018 online frei zugänglich ist, stehen Leben und Werk des österreichischen Schriftstellers Stefan Zweig (1881-1942). ${ }^{3}$ Nicht zuletzt durch die vielfachen Stationen von Zweigs Exil ergab sich für die von inm hinterlassenen Dokumente eine Verteilung auf zahlreiche Sammlungen in aller Welt und eine entsprechend heterogene Überlieferungslage. In der Folge standen die Originaldokumente von einem der auflagenstärksten und umfassendst vernetzten Autoren des 20. Jahrhunderts trotz anhaltendem Interesse an seinem Werk der wissenschaftliche Community bisher nur eingeschränkt zur Verfügung. Im Rahmen des Projekts SZD tritt das Literaturarchiv Salzburg, Forschungszentrum von Universität, Land und Stadt Salzburg, das bedeutende Originalmaterialien von und über Stefan Zweig aufbewahrt, diesen Schwierigkeiten entgegen: Erstmals werden die erhaltenen Manuskripte Zweigs sowie zugehörige Materialien systematisch online verzeichnet sowie als digitale Faksimiles präsentiert. Für die Recherche auf der Plattform stehen neben den Katalogen zu Werkmanuskripten und Lebensdokumenten eine biographische Übersicht sowie ein Index der Personen und Standorte mit über 700 Einträgen zur Verfügung. Hinzu kommt ein Verzeichnis der erhaltenen Bücher aus Zweigs Bibliothek, das einen wichtigen Einblick in die von inm wahrgenommene, gelesene und als Quelle für seine Werke genutzte Literatur bietet. ${ }^{4}$ In Kooperation mit dem Zentrum für Informationsmodellierung (ZIM) der Universität Graz sowie internationalen Partnern wie der Daniel A. Reed Library in Fredonia und der National Library of Israel wird der Nachlass des Schriftstellers Stefan Zweig als virtuelle Nachlassrekonstruktion der Öffentlichkeit zugänglich gemacht.

\section{Zur technischen Basis}

Eingebettet in das "Geisteswissenschaftliche Asset Management System" (GAMS), ein OAIS-konformes, FEDORA-basiertes Repository zur Langzeitarchivierung und Publikation von Forschungsdaten in den Geisteswissenschaften, existieren Workflows zur Standardisierung, Formalisierung und semantischen Anreicherung der Nachlassobjekte und ihrer Metadaten in einem digitalen Repositorium. ${ }^{5}$ Neben einer weitgehend XML-basierten Inhaltsstrategie zur Sicherstellung des Zugriff und der Langzeitarchivierung verwendet GAMS etablierte Metadatenstandards (TEI, METS), Datenformate (XML, RDF) und Programmierschnittstellen (IIIF). Alle digitalen Objekte im System erhalten eine persistente Kennung auf Basis des „Handle“-Systems, um die Zitation zu gewährleisten. Eine weitere Standardisierung wird durch die Verwendung des "International Image Interoperability Framework“ (IIIF) zur Verwaltung und Veröffentlichung digitaler Faksimiles erreicht.

Im Rahmen des Projektes werden erfasste Daten in TEI modelliert. METS dient als Standard zur Formalisierung digitalisierter Manuskripte und ihrer strukturellen Metadaten, die über IIIF in GAMS angeboten werden. Normalisierungen von vorkommenden Named Entities umfassen Personen (GND), Organisationen (GND), Orte (GeoNames) und spezifische Provenienzmerkmale (SKOS). Darüber hinaus werden Verknüpfungen zu Wikidata.org hergestellt. Beim Ingest der TEI/XML-Dokumente, beispiels-

3 Als verwandte Projekte, die in den letzten Jahren in Österreich entstanden sind, wären etwa die Plattformen Handke online (https://handkeonline.onb.ac.at/), Karl Kraus Online (https://www.kraus.wienbibliothek.at/) und Ernst Jandl Online (jandl.onb.ac.at/) zu nennen.

4 Stephan MATthiAS / Oliver MATUSCHEK, Stefan Zweigs Bibliotheken, Dresden 2018.

5 Vgl. Johannes STIGLER / Elisabeth STEINER, GAMS - Eine Infrastruktur zur Langzeitarchivierung und Publikation geisteswissenschaftlicher Forschungsdaten, in: Mitteilungen der Vereinigung Österreichischer Bibliothekarinnen und Bibliothekare 71/1 (2018), 207-216. 
weise der Werkliste ${ }^{6}$ in die GAMS-Infrastruktur werden bestimmte Metadaten und Objektbeziehungen als RDF/XML und somit als semantische Struktur abgebildet. Die Normalisierung von Named Entities ermöglicht die semantische Anreicherungen der Daten, beispielsweise über das Tool OpenRefine, durch Wikidata.org oder durch die GND. Die Retrieval- (Volltext) und Discovery-Funktionalitäten basieren auf diesen RDF-Daten. In Übereinstimmung mit den FAIR-Datenprinzipien werden die Projektdaten als Linked Open Data (LOD) veröffentlicht und können nachgenutzt werden. ${ }^{7}$

\section{Auf dem Weg zu einer domänenspezifischen Nachlass-Ontologie}

Klassische archivarische und bibliothekarische Erschließungsmaßnahmen bilden die beste Grundlage für das Auffinden und Erforschen von Nachlassdokumenten. Sie haben aber ihre Grenzen, wenn es darum geht, die komplexen Verbindungen von Einzeldokumenten und Nachlassteilen untereinander und nach außen aufzuzeigen und das (Kontext-)Wissen der Bearbeiter*innen zu den Originalen zu formalisieren. Semantic-Web-Technologien bieten in diesem Bereich neue Möglichkeiten, solche Verbindungen, die oftmals auch für Fachwissenschaftler*innen aus der Materialautopsie allein nicht ersichtlich wären, auf Basis einer Ontologie erstmals kenntlich zu machen.

Umreißen wir etwa folgendes Beispiel: Im Zuge seiner Beschäftigung mit der Französischen Revolution schrieb Stefan Zweig als eines von vier umfangreichen Werken 1932 die Biographie Marie Antoinette Bildnis eines mittleren Charakters. Unter den von inm hinterlassenen Papieren fand sich bisher keine komplette Fassung des Manuskripts von Marie Antoinette, das als Buch in der Erstausgabe beinahe 650 Druckseiten umfasst. Allerdings sind aus mehreren Arbeitsstufen und der Korrekturphase Skizzen, einzelne Kapitel oder Fragmente davon erhalten geblieben. In Zweigs Autographensammlung, die inm wichtige Anregungen für seine literarischen Arbeiten bot, ist außerdem eine beachtliche Zahl von Handschriften aus dem Umfeld Marie Antoinettes und der Zeit der Französischen Revolution nachweisbar. Marie Antoinette wurde eines der meistverkauften Bücher des Jahres und noch im Erscheinungsjahr der Erstausgabe in zahlreiche Sprachen übersetzt. Wie anderen Werken Zweigs haben sich auch zu Marie Antoinette Verträge mit ausländischen Verlagen erhalten. Zur besseren Übersicht über die Vermarktung seines weltweit vertriebenen Werkes wurde ab August 1932 im Büro des Autors das so genannte Hauptbuch angelegt, in dem auf vorgedruckten Doppelseiten die verkauften Rechte für jedes Werk und die dafür in Verträgen vereinbarten Konditionen mit Verlagen, Übersetzer*innen und Filmstudios festgehalten wurden. Auch eine Doppelseite zu Marie Antoinette ist darin zu finden. Unter den Bänden in Zweigs Bibliothek sind keine Quellenwerke für Marie Antoinette mehr vorhanden, allerdings liegen zahlreiche Belegexemplare von Übersetzungen des Buches als sogenannte „Hausexemplare" vor.

6 TEI/XML der Werkliste, online unter: http://stefanzweig.digital/o:szd.werke/TEI_SOURCE (12.06.2019).

7 RDF/XML Serialisierung der Werkliste, online unter: http://stefanzweig.digital/o:szd.werke/RDF (12.06.2019). 


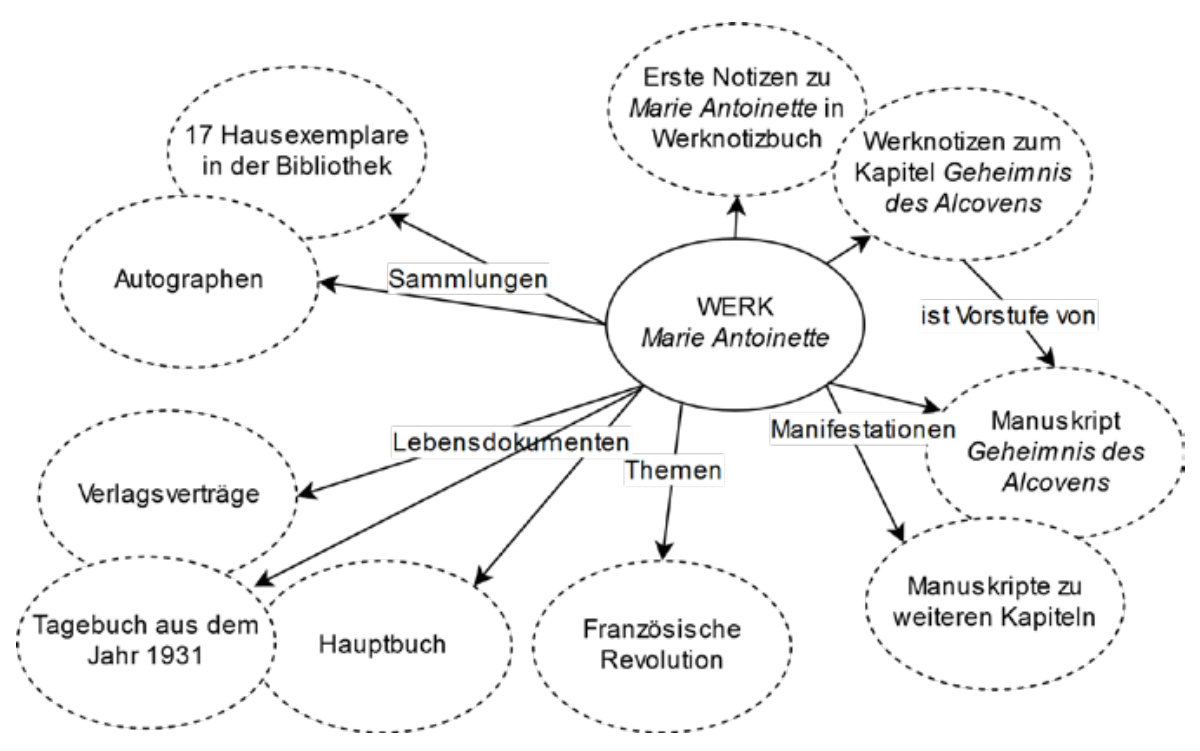

Abb. 1: Verbindungen rund um Zweigs Werk Marie Antoinette

Das Wissen um die hier beschriebenen Verbindungen kann nach der ausführlichen Erschließung jedes erhaltenen Objekts aus archivarischer und geisteswissenschaftlicher Perspektive durch die Modellierung semantischer Strukturen und Bereitstellung als LOD menschen- und maschinenlesbar zugänglich gemacht werden. ${ }^{8}$ Die Formalisierung auf Basis einer domänenspezifischen NachlassOntologie kann den hierfür notwendigen formalen und kontextuellen Rahmen schaffen.

Das formale Modell der Ontologie ist dabei auf gängige Standards wie RNAB ${ }^{9}$, die GND-Ontologie ${ }^{10}$, $\mathrm{RiC}^{11}$ und CIDOC-CRM ${ }^{12}$, sowie FRBRoo ${ }^{13}$ referenzierbar. Im Kontext des zur Verzeichnung von Archivgut entwickelten neuen Standards Records in Contexts (RiC) werden Überlegungen zur semantischen Interoperabilität in der Archivwelt diskutiert und umgesetzt. Ziel ist es, unter Berücksichtigung der Beschreibung durch etablierte Standards ${ }^{14}$ in der Archivwelt, die hierarchischen Organisationsstrukturen in Archiven aufzubrechen und Archivalien in unterschiedlichen Kontexten abzubilden. Diese Anstrengungen werden durch RiC-O als Ontologie formalisiert, ${ }^{15}$ die auch für Nachlässe nutzbar ist. ${ }^{16}$ CIDOC-CRM ist bereits ein etabliertes semantisches Framework im Semantic Web, das als gemeinsame Sprache' dazu dienen soll, Kulturerbeobjekte und ihre Dokumentation zu beschreiben, um deren konzeptionelle Interoperabilität sicherzustellen. Einzelne Spezifikationen und Erweiterungen von CIDOC-CRM, die für bestimmte Anwendungsbereiche entwickelt wurden, fördern die semantische

$8 \quad$ Eine wesentliche Herausforderung im Bereich der Forschungsdaten ist es, Datensilos zu vermeiden. Mit dem Semantic Web wird ein Technologie-Stack angeboten, um die von Tim Berners-Lee definierten Linked Open Data (LOD)-Paradigmen umsetzen zu können. Vgl. Krzysztof JANOWICZ u. a., Five stars of linked data vocabulary use, in: Semantic Web 5/3 (2014), 173-176.

9 Ressourcen-Erschließung mit Normdaten in Archiven und Bibliotheken, online unter: https://nbn-resolving.org/urn:nbn:de:101-2019051405 (10.06.2019).

10 GND Ontology, online unter: https://d-nb.info/standards/elementset/gnd (12.02.2019).

11 Records in Contexts - Conceptual Model, online unter: https://www.ica.org/en/egad-ric-conceptual-model (01.06.2019).

12 Definition of the CIDOC Conceptual Reference Model, online unter: http://www.cidoc-crm.org/Version/version-6.2.5, Version. 6.2.5, Stand Februar 2019.

13 Object-oriented Functional Requirements for Bibliographic Records, online unter: http://www.cidoc-crm.org/frbroo/homeo, Version 3.0, Stand Oktober 2017.

$14 \operatorname{ISAD}(\mathrm{G})$, ISAAR(CPF), ISDF und ISDIAH.

15 Llanes-Padrón DUNIA/ Juan-Antonio PASTOR-SANCHEZ, Records in contexts: the road of archives to semantic interoperability, in: Program 51/4 (2017), 387-405.

16 Philipp MESSNER, Records in Contexts - vom Baum zum Netz, in: Arbido 3 (2017), online unter: https://arbido.ch/de/ausgaben-artikel/2017/metadaten-datenqualit\% $\mathrm{C}_{3} \% \mathrm{~A}_{4}$ t/records-in-contexts-vom-baum-zum-netz (12.06.2019). 
Modellierung unterschiedlichster Ressourcen. Eine für SZD relevante Spezifikation ist FRBRoo. Dabei handelt es sich um eine Ontologie mit dem Ziel, den Austausch zwischen biographischen und musealen Informationen zu erleichtern.

Solcherart kann eine Nachlass-Ontologie nicht nur als Wissensbasis begriffen werden, sondern auch als konzeptionelle Grundlage für die Nutzung und Bereitstellung von Linked Open Data sowie darauf fußender erweiterter Funktionalitäten zum „digitalen Entdecken“, dem Resource Discovery. ${ }^{17}$ Diese Funktionalitäten folgen dem Paradigma der Exploratory Search, ${ }^{18}$ die Joemon Jose mit dem Satz "I don't know what l'm looking for, I'll know it when I find it ${ }^{\text {"1919 }}$ auf den Punkt bringt. Die Formulierung von ,klassischen' Suchanfragen kann nur dann geschehen, wenn ein gewisses Vorwissen über einen Bestand vorhanden ist. SZD versucht beispielsweise über biographische und thematische Einstiegspunkte solches Wissen zu vermitteln.

Trotz der Herausforderungen, die in der Wartung, Verknüpfung und Abfrage des Semantic Web liegen, erlauben es eine domänenspezifische Ontologie und die Bereitstellung als LOD, die Daten und ihre Kontexte - im Fall von SZD also die Objekte des Nachlasses und das Wissen über sie - so zu formalisieren, dass sämtliche Struktur den Daten immanent ist. Das heißt die Daten ,wissen' so viel über sich selbst, dass sie durch andere Menschen oder Maschinen automatisiert weiterverarbeitet werden können. So kann der materialreiche und inhaltlich vielfältige Nachlass Stefan Zweigs in einen wesentlich weiter gefassten Zusammenhang des kulturellen und intellektuellen Lebens seiner Zeit gestellt werden.

\section{Literaturverzeichnis}

Llanes-Padrón DUNIA / Juan-Antonio PASTOR-SANCHEZ, Records in contexts: the road of archives to semantic interoperability, in: Program 51/4 (2017).

Krzysztof JaNOWICZ u.a., Five stars of linked data vocabulary use, in: Semantic Web 5/3 (2014).

Joemon M. Jose, Personalisation Techniques in Information Retrieval, in: DigiCULT 6 (2004).

Stephan MatTHiAs / Oliver Matuschek, Stefan Zweigs Bibliotheken, Dresden 2018.

Philipp Messner, Records in Contexts - vom Baum zum Netz, in: Arbido 3 (2017), online unter: https://arbido.ch/de/ausgaben-artikel/2017/metadaten-datenqualit\%C3\%A4t/records-in-contexts-vom-baum-zum-netz (12.06.2019).

Johannes STIGLER / Elisabeth STEINER, GAMS - Eine Infrastruktur zur Langzeitarchivierung und Publikation geisteswissenschaftlicher Forschungsdaten, in: Mitteilungen der Vereinigung Österreichischer Bibliothekarinnen und Bibliothekare 71/1 (2018), 207-216.

Dirk WEISBROD, Langzeitarchivierung digitaler Schriftstellernachlässe. Eine Bestandsaufnahme in den Literaturarchiven Deutschlands, Österreichs und der Schweiz, in: Young Information Scientist 2 (2017), online unter: http://eprints.rclis.org/30976/ (12.06.2019).

Ryen WHITE / Resa A. Roth, Exploratory search: beyond the query-response paradigma, San Rafael, Calif. 2009.

17 Beispiele für solche konkreten Anwendungsszenarien, die es erlauben, formalisierte semantische Strukturen zu nutzen, finden sich etwa in den Projekten ResearchSpace des British Museum (https://www.researchspace.org) oder Burghardt Source (http://burckhardtsource.org).

18 Ryen WHITE / Resa A. RotH, Exploratory search: beyond the query-response paradigma, San Rafael, Calif. $2009,14$.

19 Joemon M. Jose, Personalisation Techniques in Information Retrieval, in: DigiCULT 6 (2004), 22. 


\title{
Identität und Normdaten
}

\author{
Joseph Wang \& Ulrich Lobis
}

Forschungsinstitut Brenner-Archiv, Universität Innsbruck

joseph.wang@uibk.ac.at, ulrich.lobis@uibk.ac.at

https://www.uibk.ac.at/brenner-archiv/

Keywords: Identity, Ontology, Controlled Vocabulary, Analogy, RDF, Semantic Web

\begin{abstract}
This paper shows why „identification“ (resp. „re-identification“) and medieval philosophy play a major role in the usage of authority-controlled vocabulary. Furthermore, we explain why the connection of knowledge from different sources - the main idea of the semantic web - is problematic.
\end{abstract}

In diesem Beitrag erläutern wir, warum die Handlung „Identifizieren“ (bzw. „Re-Identifizieren“) und die mittelalterliche Sprachphilosophie eine wesentliche Rolle in der Verwendung von Normdaten zur Beschreibung der eigenen Datensätzen spielen. Darüber hinaus erklären wir, warum das Ideal des semantic webs, Wissen aus unterschiedlichen Quellen miteinander in Beziehung zu setzen, um möglichst viel Wissen zu generieren, problematisch sein kann.

Den Ausgangspunkt unserer Überlegung bilden die im Alltag eingeübten Handlungen Identifizieren und Re-Identifizieren.

\section{Identifizieren und Re-Identifizieren}

Die Vollzüge Identifizieren und Re-Identifizieren spielen bei allen unseren Alltagshandlungen eine sehr wichtige Rolle: Wollen wir ein Kind für seine Tat loben, so müssen wir es identifizieren und re-identifizieren. Wenn wir die Arbeit an einem Artikel nach einer Pause wieder aufnehmen wollen, müssen wir die Datei wiederfinden. Wird uns die Aufgabe gestellt, sieben Äpfel und fünf Birnen einzukaufen, müssen wir in der Lage sein, Äpfel und Birnen voneinander zu unterscheiden. Diese scheinbar einfache Handlung des „Identifizierens" setzt aber unglaublich viel Wissen voraus, das Menschen im Alltag als selbstverständlich erachten, das Philosoph*innen aber viele Fragen aufgibt.

Wir können hier nicht auf alle Einzelheiten der Identifikation eingehen. Stattdessen setzen wir voraus, dass Menschen im Alltag normalerweise kein Problem damit haben, eine Person von einer anderen zu unterscheiden, dass sie normalerweise problemlos Dinge bestimmter Art (z. B. Äpfel, Hefte, Stifte) zählen können, und dass sie normalerweise keine Probleme haben, Städte, Fußballspiele, wissenschaftliche Artikel, Bilder, Lieder, Dateien und RDF-Aussagen voneinander zu unterscheiden und entsprechend ihrer Identifikation dieser Entitäten erfolgreich zu handeln. Weiter setzen wir voraus, dass sie eher Schwierigkeiten haben, bestimmte Entitäten zu identifizieren, z. B. Abstrakta wie Liebe (auch wenn wir Liebespaare zählen können), Betrug (im Gegensatz zu Betrugsfällen) oder auch sehr allgemeine Termini. 


\section{Identifizieren und Re-Identifizieren in Semantic Web Technology}

Dass die Handlungen Identifizieren und Re-Identifizieren auch bei der Erstellung und Anwendung von Norm-Daten, die als RDF-Tripeln vorliegen, eine große Rolle spielen, verwundert nicht. Betrachten wir das folgende RDF-Statement:

$$
\text { URI: „Österreich“ --- URI: „hat Präsident“ --> URI „Alexander van der Bellen“ }
$$

Wollen wir dieses RDF-Statement machen, müssen wir in der Lage sein, ein Land als „Österreich“ und eine Person als „Alexander van der Bellen“ zu identifizieren. Darüber hinaus müssen wir in der Lage sein, „hat Präsident“ als dasselbe Prädikat zu identifizieren, das auch bei RDF-Aussagen über Staatsoberhäupter verwendet wird. Mit anderen Worten: Damit RDF und die Semantic Web Technology (SWT) funktionieren, müssen die Leistungen Identifizieren und Re-Identifizieren bereits erbracht sein. Die einzige Regel bezüglich des Identifizierens, mit der RDF operiert, ist die folgende: „Wenn zwei URIs gleich lauten, dann sind sie miteinander identisch“."

Wir glauben, dass diese Regel die eigentliche „Stärke“ von SWT ist. Wenn wir (propositionales) Wissen als RDF-Statements repräsentieren, können wir dank dieser Möglichkeit des Identifizierens einer Entität automatisch Wissen generieren lassen. Wir können dies auch dann tun, wenn die RDF-Statements aus unterschiedlichen Datenquellen stammen.

Nehmen wir an, dass eine Datenquelle folgendes feststellt:

URI: „Österreich“ --- URI: „hat Präsident“ --> URI „Alexander van der Bellen“.

Und nehmen wir weiter an, dass die Datenquelle B folgendes feststellt:

URI: „Alexander van der Bellen“ --- URI: „hat Geschlecht“ --> URI: „männlich“,

so kann ein Computer-Programm daraus schließen, dass das Geschlecht des Präsidenten von Österreich männlich ist. Bekommt man diese Informationen aus unterschiedlichsten Quellen, kann man bspw. auflisten, welche Länder der Welt einen Präsidenten haben, der (nicht) männlich ist.

Klarerweise kann es vorkommen, dass unterschiedliche Datenquellen für dieselbe Entität unterschiedliche URIs vergeben. Damit der Zusammenschluss der Datenquellen dennoch gut funktioniert, liefert die SWT ein besonderes Prädikat mit: „owl:sameAs“. Stellt jemand fest, dass

$$
\text { URI:A --- owl:sameAs --> URI:B }
$$

ist, dann kann man alle Aussagen über A auch auf $B$ übertragen.

Es ist naheliegend, dass der größte Nutzen des owl:sameAs-Prädikats in erster Linie dort zu finden ist, wo zwei verschiedene Semantic Web Ontologies miteinander verknüpft werden sollen.

1 Vgl. W3C, RDF 1.1 Concepts and Abstract Syntax (Introduction), online unter: https://www.w3.org/TR/rdf11-concepts/ (08.04.2019). 


\section{Probleme der Identitätsaussagen}

Grundsätzlich geht man davon aus, dass man identische Begriffe in einer Aussage miteinander salva veritate tauschen kann und dabei den Wahrheitswert, also den Wahrheitsgehalt des Satzes nicht ändert. Ein Beispiel wäre etwa, „Kochsalz ist wasserlöslich“ und „N $\mathrm{NaCl}$ ist wasserlöslich“. Beide Sätze sind inhaltlich nicht voneinander verschieden, was an der Identität zwischen den Wörtern Kochsalz und $\mathrm{NaCl}$ liegt.

Dass die Übertragung der Aussagen nicht immer so reibungslos ist, sieht man bereits am folgenden Beispiel: Man stelle sich zwei Datenquellen vor, wobei die eine die jüngere Geschichte Südamerikas bis 2010 behandelt und in der Jorge Mario Bergoglio vorkommt; in der anderen wird die Geschichte des Papsttums behandelt, und in dieser kommt Papst Franziskus vor. Nun ist es so, dass Bergoglio der bürgerliche Name von Papst Franziskus ist, d. h. dieselbe Person wird mit beiden Namen identifiziert. Es wäre folglich vollkommen intuitiv zu sagen, „set1:Jorge Mario Bergoglio owl:sameAs set2:Papst Franziskus“. Allerdings kommt man dadurch sehr schnell in die Bredouille: Zwar sind die Sätze: „Jorge Mario Bergoglio ist 201881 Jahre alt“ und „Papst Franziskus ist 201881 Jahre alt“ gleich, aber mit den Sätzen „Jorge Mario Bergoglio ist unfehlbar“ und „Papst Franziskus ist unfehlbar“ verhält es sich komplizierter, denn die Unfehlbarkeit wird speziell dem Papst zugesprochen, nicht aber dem Priester oder Bischof Bergoglio. Noch offensichtlicher wird es hingegen, wenn man die andere Richtung geht, und den Satz „Jorge Mario Bergoglio ist ein braves Kind“ und die Ersetzung, „Papst Franziskus ist ein braves Kind“ ansieht.

Wenn man das Problem studiert, kann man folgende Struktur ausmachen: Es gibt hier zwei Individuen, von denen man common sense sagen kann, dass sie identisch sind. Es stellt auch im normalsprachlichen Kontext keine Schwierigkeit dar, mit der Identität zu arbeiten und sie richtig zu verwenden. Hingegen beginnen die Probleme dort, wo diese Identität als Grundlage für die Bildung neuer Sätze verwendet wird.

Wie kommt es zu diesem Problem? Warum gelten die logischen Regeln für Identität nicht auch im Altag? Hier wollen wir zwei Gründen genauer nachgehen.

\section{Identitätsaussagen sind kontextabhängig}

Am Beispiel der Identität des Papstes mit Bergoglio - aber auch am Beispiel der Identitätsaussage, dass der Abendstern identisch mit dem Morgenstern sei - sieht man, dass der Wahrheitswert der Identitätsaussagen auch vom Kontext der Aussage abhängt. Wir wissen heute, dass der Planet Venus sowohl der Abend- als auch der Morgenstern ist. Im Kontext, in dem wir nach der Entfernung des Abendsterns von der Sonne und nach der Entfernung des Morgensterns von der Sonne fragen, können wir - dank der Identitätsaussagen der beiden Sterne mit Venus - mit der Entfernung der Venus von der Sonne antworten. Hat man allerdings ein Treffen in der Nacht, das mit Anbruch der Morgenröte endet, würde die Aussage, ob der Stern am Horizont nun der Abend- oder der Morgenstern ist, eine andere Bedeutung bekommen.

\section{Die Aussage „x ist identisch mit y“ muss analog verstanden werden}

Neben der Kontext-Abhängigkeit ist die analoge Verwendungsweise des Begriffs „Identität“ ein wichtiger Aspekt im Verständnis von Identitätsaussagen. 
Begrifflich hilft es, sich hier der Scholastik, namentlich Thomas von Aquin, zuzuwenden. ${ }^{2}$ Thomas greift den Begriff der Analogie auf und vergleicht inn mit Univozität und Äquivokation. ${ }^{3}$ Während etwas als univok bezeichnet wird, wenn derselbe Name und dieselbe Natur (ratio) von einer Sache ausgesagt wird (sowohl von einem Menschen als auch einem Esel wird ausgesagt, dass er ein Tier ist) und etwas als äquivok bezeichnet wird, wenn es zwar denselben Namen verwendet, aber eine gänzlich andere Natur meint (der Hund als Lebewesen als auch das Sternbild des Hundes), liegt der Begriff der Analogie dazwischen. Für Thomas wird etwas dann analog verwendet, wenn ein Begriff von verschiedenen Naturen ausgesagt wird, aber dennoch eines von allen ausgesagt wird (,analogice dicitur praedicari, quod praedicatur de pluribus quorum rationes diversae sunt sed attribuntur uni alicui eidem"). Das klassische Beispiel ist "gesund“, das von verschiedenen Dingen ausgesagt werden kann (dem Menschen, dem Blutbild, dem Apfel), und bei allen etwas verschiedenes meint, aber dennoch in einem „Ende“, der Gesundheit, zusammenläuft („sed tamen omnes istae rationes attribuuntur uni fini, scilicet sanitati“).

Im Sentenzenkommentar ${ }^{4}$ führt Thomas eine Variante an, die für diesen Fall interessant ist: Es gibt eine Analogie sowohl im Sein als auch in der Begrifflichkeit (secundum intentionem et secundum esse), die das Historische Wörterbuch der Philosophie expliziert als etwas das „vorliegt, wenn im Nachgeordneten etwas vom Sein des Vorgeordneten bestimmend anwesend ist, aber in minderer Vollkommenheit, so daß es nicht voll die ,ratio' des Begriffs erfüllt - in diesem Sinne ist Wahrsein und Gutsein sowohl in Gott als in den Geschöpfen und kann von beiden analog ausgesagt werden." ${ }^{5}$

Dies kann hilfreich sein, wenn man es auf den Begriff der Identität anwendet. Identität im vollen Sinne kommt nur einer Sache im leibnizschen Sinne zu, das heißt identisch ist eine Sache nur mit sich selbst - oder wenn man im Sinne der Scholastik spricht, nur Gott. Wenn man also die Verwendung des Identitätsoperators im Alltag betrachtet, findet man viele Fälle, in denen nicht im Vollsinn von Identität gesprochen wird. Identität ist hier meist nur in einer schwachen Form vorhanden, aber dies fällt weder auf noch stört es, da wir gewohnt sind, damit umzugehen.

\section{Konklusion: Bei Ontologien sollte man in den meisten Fällen auf Identitätsaussagen verzichten}

Welche Schlüsse kann man aus der Annahme, dass die Identitätsaussagen kontext-abhängig und in analoger Weise zu verstehen sind für die SWT ziehen?

Es gibt Autor*innen, die für die Einführung eines fein-granulareren Vokabulars plädieren. Sie führen Prädikate wie „identisch“, „ähnlich“, „trifft zu“ bzw. „verbunden“ ein, die sich in ihrer Transitivität, Symmetrie und Reflexivität unterscheiden. Statt „A ist identisch mit B“ soll man nun ein Statement in der Form „A ist ähnlich wie B“ machen. Dadurch kann man nur noch wenige Aussagen von B salve veritate für A übernehmen. ${ }^{6}$

2 Vgl. Wolfgang KLuXen / Adolf Remane, Analogie (I, 6), in: Joachim Ritter / Karlfried Gründer / Gottfried Gabrielm, Hg., Historisches Wörterbuch der Philosophie, DOI: 10.24894/HWPh.5036 (08.04.2019).

3 Vgl. Thomas von Aquin, De princ. nat. c. 6.

4 Vgl. Thomas von Aquin, In I. Sent. 19, 5, 2 ad 1.

5 KLuXen / Remane, Analogie (l, 6).

6 Vgl. Harry Halpin u. a., When owl:sameAs isn't the same: An analysis of identity in linked data, in: Peter F. Patel-Schneider u. a., International semantic web conference -ISWC 2010. gth International Semantic Web Conference 2010, Berlin I Heidelberg 2010, 305-320, hier besonders 311-313. 
Die Problematik bei diesem Vorschlag dürfte allerdings darin liegen, dass damit weder das Problem der Kontext-Abhängigkeit noch jenes der analogen Verwendungsweise gelöst werden, aber durch die Einführung der neuen Prädikate wird der Umgang mit ihnen erheblich verkompliziert.

Dank der SWT bieten uns diverse Datengeber*innen ihre Daten aber genau zum Verknüpfen an. Sowohl die Normdaten als auch Open Government Data sind so angelegt, dass sie weitergenutzt werden. In unserer eigenen Forschung wird „owl:sameAs“ dazu verwendet, um auf Normdaten zu verweisen. Die oben beschriebenen Probleme zeigen aber, dass der Zusammenschluss von Datenquellen nur schwer umsetzbar ist. Müssten wir demnach auf die Verwendung von Normdaten, bzw. auf die Publikation von Aussagen mit „owl:sameAs“ verzichten? Hierzu möchten wir auf zwei Arten antworten:

\section{Die Nutzung fremder Datenquellen ist erlaubt, wenn die Datenquellen überprüft worden sind}

Was sind typische „Use-Cases“, in denen fremde Datenquellen verwendet werden? Schon bevor Computer für die Forschung eingesetzt wurden, wurden fremde Quellen vor ihrer Nutzung überprüft. Stichworte wie "Quellen-Kritik“ oder „fake news" machen darauf aufmerksam, dass nicht alles übernommen werden darf, was gesagt oder geschrieben wurde. Bei der Verwendung einer Datenquelle für die Forschung in den Geisteswissenschaften gilt wohl das gleiche. Bereits Thaller hat darauf hingewiesen, dass vor der Verwendung einer Datenquelle darauf geachtet werden muss, wie die Daten in ihr erfasst worden sind und ob die verwendete Methode für die aktuelle Forschungsfrage Geltung haben kann. ${ }^{7}$

Lässt der Kontext der aktuellen Forschungsfrage zu, dass bestimmte Statements von anderen Datenquellen übernommen werden? Das Prädikat „owl:sameAs“ könnte zu stark sein, aber die kontrollierte Übernahme bestimmter Statements könnte dennoch zulässig sein.

\section{Konsequenz für Normdaten}

Die Normdaten sind per definitionem dazu da, um Daten aus unterschiedlichen Quellen zusammenzuschließen. Wenn Forscher*innen die Daten nur kontrolliert übernehmen sollen, wozu dienen die Normdaten und vor allem die vielen Verweise auf sie dann?

Freilich tauchen die oben beschriebenen Probleme bei der Erstellung von Normdaten auf, insbesondere ist es nicht immer einfach, Meta-Normdaten (wie jene aus VIAF) zu erstellen. Jedoch muss festgehalten werden, dass Normdaten, die in Bibliotheken verwendet werden, einen eingegrenzten (und relativ einfachen) Kontext haben: Sie sind da, damit Benutzerinnen und Benutzer von Katalogen die Bücher, Artikel oder andere Medien leichter finden können. Für diesen Zweck wurden die Normdaten erschaffen. Und falls wir Normdaten publizieren, sollte unser Augenmerk ebenfalls darauf ausgerichtet sein.

7 Vgl. Manfred THALLER, Ungefähre Exaktheit: theoretische Grundlagen und praktische Möglichkeiten einer Formulierung historischer Quellen als Produkte ,unscharfer' Systeme, in: Historical Social Research, Supplement 29 (2017), 138-159, hier besonders 142-144. 


\section{Literaturverzeichnis}

Harry HaLPIN u. a., When owl:sameAs isn't the same: An analysis of identity in linked data, in: Peter F. Patel-Schneider u. a., International semantic web conference -ISWC 2010. 9th International Semantic Web Conference 2010, Berlin / Heidelberg 2010, 305-320, hier besonders 311-313.

Wolfgang KLUXEN / Adolf REMANE: Analogie (I, 6), in: Joachim Ritter / Karlfried Gründer / Gottfried Gabrielm, Hg., Historisches Wörterbuch der Philosophie, DOI: 10.24894/HWPh.5036 (08.04.2019).

Manfred THALLER, Ungefähre Exaktheit: theoretische Grundlagen und praktische Möglichkeiten einer Formulierung historischer Quellen als Produkte ,unscharfer' Systeme, in: Historical Social Research, Supplement 29 (2017), 138-159.

W3C, RDF 1.1 Concepts and Abstract Syntax (Introduction), online unter: https://www.w3.org/TR/rdf11-concepts/ (08.04.2019). 


\title{
Zwei Überlegungen zur Konzeption einer Linked-Data-Ontologie für die Literaturwissen- schaften
}

\author{
Bernhard Oberreither \\ ÖAW, ACE - Austrian Corpora and Editions \\ bernhard.oberreither@oeaw.ac.at
}

Keywords: Linked Open Data, Semantic Web, Literary Studies, Intertextuality, Ontology

\begin{abstract}
The paper outlines reflections on constructing a Linked Data ontology for literary studies, especially intertextuality. In particular, a case is made for two modeling principles - textualism and implicitness of methodological specifics -, which are briefly discussed with regard to different demands to DH made by proponents as well as critics of this field of research.
\end{abstract}

Vorbemerkung: Im Folgenden werden - in aller Kürze - Überlegungen vorgestellt, die der Konzeption der Ontologie INTRO („Intertextual Relationships Ontology“) vorausgingen. Die Ausführungen gehen auf einen Vortrag auf der dha2018 zurück, beschränken sich dabei jedoch auf einen Teil der damals behandelten Inhalte. Einen Überblick über INTRO selbst sowie Beispielmodellierungen finden sich im readme auf GitHub. ${ }^{1}$

\section{I.}

For nearly six centuries, humanistic models of knowledge have been shaped by the power of print as the primary medium of knowledge production and dissemination. Rather than rejecting print culture or embracing the simple pouring of print models into digital molds, Digital Humanities is engaged in developing print-plus and post-print models of knowledge. Both involve more than an updating of the knowledge delivery system. They entail the cognitive and epistemological reshaping of humanistic fields as a function of the affordances provided by the digital with respect to print. $^{2}$

- so eine Definition der digitalen Geisteswissenschaften in der jüngeren Einführungsliteratur. Digitale Geisteswissenschaften, kann man dem entnehmen, seien kein bloßes Update, vielmehr eine kognitive und epistemologische Neu- oder Umgestaltung der Geisteswissenschaften, und gehen also, wie sich daraus schließen lässt, weit über den Einsatz von Textverarbeitungs-Programmen und die Gestaltung von informativen Projekt-Websites hinaus. Passagen wie diese sind natürlich normativ mehr denn deskriptiv, oder, anders ausgedrückt: Beschwörungen eher als Beschreibungen. Wer in den digitalen

1 Vgl. Bernhard OBERREITHER, INTRO. An Intertextual Relationships Ontology for literary studies, online unter: https://github. com/BOberreither/INTRO (16.04.2019).

2 Ann BuRDick u. a., Digital_Humanities, Cambridge (Mass.) / London 2012, 125. 
Geisteswissenschaften publiziert, landet bald in diesem Register, das gilt natürlich auch für den Bereich Linked Data (und schließt den vorliegenden Text mit ein). Zu bedenken ist dabei, dass des einen Wunschvorstellung des anderen Schreckensvision sein kann. In seiner schon aufgrund ihres hohen Unterhaltungsfaktors sehr lesenswerten Breitseite gegen die Digital Humanities und in direkter Bezugnahme auf die oben zitierte Publikation schreibt Jeremy Brennan 2017:

The authors summarize the intellectual activities they promote: "digitization, classification, description and metadata, organization, and navigation." An amazing list, which leaves out that contradictory and negating quality of what is normally called "thinking". It would be a mistake to see this banishment of the concept as the passive by-product of a technical constraint. It is the aim of the entire operation. ${ }^{3}$

Das Denken gehe am Übergang zu den digitalen Geisteswissenschaften verloren, und abseits der Polemik kann man dem immerhin soweit folgen, dass Textarbeit bei der Anwendung etwa von text mining, topic modeling etc. nicht mehr primär der humanen agency obliegt. Der Fluchtpunkt solcher und ähnlicher Einwürfe liegt wohl in der Frage: Angenommen, Kulturwissenschaft ist von ihrem Gegenstand nicht getrennt, sondern tatsächlich Teil von Kultur, angenommen außerdem, dass Menschen die zentralen Akteure von Kultur sind - welchen Stellenwert kann dann ein Ergebnis beanspruchen, das nicht-humaner agency entspringt?

Auf der anderen Seite stehen Vertreter*innen der digitalen Geisteswissenschaft, die mit Recht auf einige Unzulänglichkeiten ihres traditionell arbeitenden Gegenübers hinweisen: auf die oft mangelhafte Nachvollziehbarkeit der Datenverarbeitung (also des Denkens), die oftmals geringe Datengrundlage, und, seit einiger Zeit, das, was als ,Datensilo-Problematik' bekannt ist: Forschungsergebnisse in traditionellen Publikationsformen sind (das betrifft auch digitale Formate wie Datenbanken etc.) meist nicht standardisiert, somit nicht automatisiert mit weiteren Ressourcen zu verknüpfen, und damit einer vielversprechenden Möglichkeit - nämlich der Datenakkumulation und der Abfrage aus kombinierten Datensets - beraubt. „Set your Data free!“ lautet dementsprechend der Impetus der Vertreter*innen von Linked Data, ebenso der Titel eines entsprechenden Vortrags aus dem Jahr $2017 .{ }^{4}$

Das Potenzial von Linked Data ist tatsächlich groß: So lässt sich mit dieser Technologie der Datensilo-Problematik begegnen, indem einerseits Ergebnisse maschinenlesbar und automatisiert verknüpfbar gemacht werden, und indem andererseits die oft in puncto Vorwissen voraussetzungsreichen Aussagen aus den verschiedenen Disziplinen auch an eben dieses Vorwissen, an ihre methodischen oder theoretischen Grundlagen rückgebunden werden können (um den Datensilo so ein zweites Mal zu durchstoßen, nun gewissermaßen vertikal). ${ }^{5}$

Für Datenintegration dieser Art greift Linked Data auf standardisierte Metadaten-Vokabulare zurück, die jeweils einen bestimmten Ausschnitt der Welt abbilden. Vokabulare dieser Art, sog. ,Ontologien“, können mittels eines zugleich hierarchisch und über Relationen strukturierten Klassensystems etwa auch geistes- bzw. literaturwissenschaftliche Leistungen im Semantic Web darstellen.

3 Timothy BRENNAN, The Digital-Humanities Bust. After a decade of investment and hype, what has the field accomplished? Not much, in: The Chronicle of Higher Education, 15.10.2017.

4 Vgl. Torsten SCHRADE, „Set your data free“ - Vom geisteswissenschaftlichen Datensilo zur vernetzten Wissensbasis mittels Linked Open Data. Siehe Martin PRELL, Digital Humanities in Jena, 23.11.2017 [Tagungsbericht], in: H-Soz-Kult, 27.02.2018, online unter: www.hsozkult.de/conferencereport/id/tagungsberichte-7576 (16.04.2019).

5 Vgl. Fabio CıоттI, Digital literary and cultural studies: the state of the art and perspectives, in: Between IV/8 (2014), online unter: http://ojs.unica.it/index.php/between/article/view/1392/1183 (16.04.2019). 
Bis zu einem gewissen Grad an Feinkörnigkeit kann man so, das geeignete Vokabular vorausgesetzt, tatsächlich digital festhalten, was Brennan so schmerzlich vermisst: humane agency - indem sich etwa der Inhalt traditioneller Forschungsbemühungen, der sich ansonsten beispielsweise in einem Aufsatz niederschlagen würde, in RDF formulieren ließe, und das auf eine Art und Weise, die die schon erwähnten Einschränkungen traditioneller Publikationsformen überwindet.

II.

Intertextualität und Semantic-Web/Linked-Data-Technologie scheinen wie füreinander gemacht. So wurde schon früh auf die konzeptionelle Ähnlichkeit zwischen beiden hingewiesen, ${ }^{6}$ insofern als Intertextualitätsforschung ja auch darin besteht, Relationen zwischen Entitäten zu klassifizieren; der prototypische Befund verbindet Text mit Text, also Subjekt über Relation mit Objekt. Dieser Dreischritt entspricht dem RDF-Triple. ${ }^{7}$

Die Modellierung von Intertextualität ist zudem aus zwei ganz praktischen Gründen sehr reizvoll: Der Gegenstandsbereich selbst ist entweder schon online verfügbar oder kann ohne weiteres digitalisiert werden; URI-identifizierte Ressourcen liegen zumindest im Fall bibliographischer Metadaten schon vor. ${ }^{8}$ Zudem deckt der Begriff der Intertextualität, so er voll ausgeschöpft wird, eine überraschend große Bandbreite literaturwissenschaftlicher Tätigkeiten ab. Literaturwissenschaft, die etwa Motivgeschichte, lyrische Formen, kulturelle Kontexte oder Gattungszugehörigkeit behandelt, stellt in jedem dieser Arbeitsbereiche Verknüpfungen her: zwischen Einzeltexten, zwischen Elementen innerhalb eines Textes, zwischen Einzeltexten und abstrakten Konzepten wie Gattungen oder formalen, semantischen oder rhetorischen Merkmalen. Und alles das liegt - je nach Theorie-Frame - entweder ohnehin als Text vor oder hat textuelle Grundlagen oder Repräsentanten.

Dazu kommt eine Eigenschaft, die ich behelfshalber den ,immanenten Konstruktivismus' dieser Technologie nenne (Vorschläge für eine elegantere Formulierung sind willkommen): Das Semantic Web erlaubt, Daten aus der Perspektive unterschiedlichster epistemologischer Positionen, auf unterschiedlichsten methodischen und theoretischen Grundlagen in den Blick zu nehmen. Jedes individuelle Element, über das sich Aussagen treffen lassen (technisch gesprochen: jede URI), kann einer theoretisch unbegrenzten Anzahl von Klassen aus einer ebenso unbegrenzten Anzahl von Ontologien zugeordnet werden; die Ontologien sind einsehbar (und im Idealfall gut dokumentiert), jede Aussage wird so als Interpretation erkennbar, die auf ihre eigenen Voraussetzungen zurückverweist.

Dieser für die Geisteswissenschaften so angemessen erscheinende Rahmen stellt zugleich eine Herausforderung für die Konzeption einer entsprechenden Ontologie dar: Ziel muss es ja sein, dieses Merkmal des Semantic Web auf Ebene des angestrebten Vokabulars wiederherzustellen, um es für die Beherbergung unterschiedlichster Theorieframes tauglich zu machen. Und wenn schon die bereits angesprochene Bandbreite an Tätigkeitsfeldern, in denen im weiteren Sinne intertextuell verfahren

$6 \quad$ Vgl. Joseph F. TENNIS, URIs and Intertextuality: Incumbent Philosophical Commitments in the Development of the Semantic Web, in: Knowledge Organization and the Global Information Society: Proceedings of the Eighth International ISKO Conference, Würzburg 2004, 103-108.

7 Mit CiTO liegt eine Ontologie vor, die Intertextualität auf diese Art abbilden kann: Silvio PERONı / David SHOTTON, The SPAR Ontologies. Submitted to the 17th International Semantic Web Conference, 2018, online unter: https://w3id.org/spar/article/spar-iswc2018/ (11.05.2018). Die Ontologie ist auf die Abbildung von Zitationsverhältnissen im wissenschaftlichen Diskurs ausgerichtet und damit natürlich hinsichtlich ihrer Anwendbarkeit für die Literaturwissenschaft beschränkt.

8 Zu nennen ist hier natürlich die GND, die „Gemeinsame Normdatei“ der Deutschen Nationalbibliothek (http://www.dnb. de/gnd). 
wird, groß ist, ist die Zahl an unterschiedlichen Möglichkeiten, Intertextualität theoretisch zu konzipieren, noch ungleich größer.

Es geht also darum, Universalität und Domänenspezifik zu versöhnen, das heißt in diesem Fall: eine Ontologie zu konzipieren, die einerseits die groß Bandbreite intertextueller Typen und Theorien umfasst, ohne, dass dabei andererseits die verschiedenen Theoriegebäude, auf denen die zu modellierenden Aussagen beruhen, miteinander in Konflikt geraten.

\section{III.}

Intertextualität, d. h. die Beziehung zwischen Texten, lässt sich theoretisch ganz unterschiedlich fassen, davon zeugen Ansätze, die seit 1968 unter diesem von Julia Kristeva ${ }^{9}$ geprägten Begriff kursieren sowie schon davor unter verschiedenen der Rhetorik oder der Gattungstheorie entstammenden Termini. Zwei Positionen an weit entfernten Polen des breiten Theorie-Spektrums wären etwa die der Einflussforschung auf der einen und die der Intertextualität nach Kristeva auf der anderen Seite. Die Einflussforschung interpoliert aus den Verhältnissen zweier zeitlich aufeinanderfolgender Texte ein Kausalverhältnis, das so aussieht: Ein Autor liest den Text eines anderen Autors, was wiederum seinen eigenen Schreibakt und den resultierenden Text beeinflusst. Kristeva hingegen konzipiert einen zeitund ortlosen Raum, in dem die Gesamtheit aller Texte zueinander in einem Verhältnis gegenseitiger „Absorption und Transformation"10 steht.

Schon an diesen sehr rudimentären Beschreibungen der Theoriegebäude wird deutlich, dass eine Modellierung dieser beiden Typen von Intertextualität Klassen und Relationen verlangen würde, die nicht nur unterschiedlich, sondern tatsächlich inkompatibel wären: In Kristevas Konzeption ${ }^{11}$ gibt es etwa nichts, das sinnvoll der Klasse „Ereignis“ (an einem Punkt in Zeit und Raum) zuzuordnen wäre, während hingegen (biographische, historische) „Ereignisse“ im Bereich der Einflussforschung für die Text-Text-Relation unabdingbar sind.

Die Lösung des Problems hat zwei Seiten: Sinnvoller Ausgangspunkt ist erstens der kleinste gemeinsame Nenner literaturwissenschaftlicher Arbeit. Dieser Nenner ist schlicht die Arbeit am Text. Der kleinste gemeinsame Nenner der Intertextualitätsforschung wiederum ist die Text-Text-Beziehung. Als zentrales Theorem einer entsprechenden Ontologie (und erste Hälfte der Lösung des Problems) bietet sich also der Textualismus an: insofern, als er den Gesprächsgegenstand sinnvoll auf Texte (sowie Textmerkmale) und Relationen reduziert. Andere Aspekte, die je nach Theoriegrundlage ebenso wichtig sein können, wie Autorschaft und Einfluss, Handlungen, Intentionen, Kultur, Systeme, Institutionen etc. stehen dem möglichst universellen Zuschnitt des Vokabulars im Wege. Gerade in seiner scheinbaren Beschränkung bietet der Textualismus das ideale Meta-Modell zur Integration verschiedenster Forschungsmethoden.

9 Julia KRISTEVA, Bachtin, das Wort, der Dialog und der Roman, in: Dorothee Kimmich / Rolf Günter Renner / Bernd Stiegler, Hg., Texte zur Literaturtheorie der Gegenwart, Stuttgart 2004, 334-348.

10 Ebd., 337.

11 Die jedoch gerade durch ihre eigenen Fallstudien nicht exemplifiziert werden - vgl. dazu Jonathan CULLER, Presupposition and Intertextuality, in: MLN 91/6 (1976), 1380-1396, insbes. 1384f. 
Diese lassen sich dann gewissermaßen indirekt einführen: Indem verschiedene Arten intertextueller Verhältnisse als je unterschiedliche „Typen intertextueller Relation“ formuliert werden: Alles, was über die Text-Text-Relation hinaus theoriespezifisch ist (etwa Ereignisse, Intentionen etc.), ist diesem „Typ“ implizit. ${ }^{12}$

\section{IV.}

Damit sind zwei grundlegende Bausteine für eine Ontologie benannt, mit der man über Texte, Textmerkmale und, darauf aufbauend, über intertextuelle Relationen sprechen können soll: der Textualismus einerseits sowie die Implizitheit methodisch-theoretischer Spezifika andererseits.

Man könnte sich abschließend fragen, ob eine Ontologie, deren Konzeption die eben besprochenen Punkte beachtet, den Forderungen entspricht, die eingangs von Burdick und Brennan aufgestellt wurden. Die Antwort wäre hinsichtlich Brennans impliziter Forderung nach der Abbildung menschlicher agency recht eindeutig: Diesem Anspruch wird eine Ontologie wohl kaum gerecht, die im „Typ intertextueller Relation“ die theoretischen Implikationen - den Kern menschlicher Agency, des Denkens! - auf eine einzige Instanz komprimiert (deren Details sich dann ggf. nur in einem Literal nachlesen lassen).

Resultiert darüber hinaus die Übertragung von Forschungsergebnissen in Linked Data nicht in einem bloßen ,Update des Wissens-Übertragungssystems' - also in dem, was Burdick et al. explizit aus den ,eigentlichen' Digital Humanities ausgeschlossen hatten? Das hängt natürlich vom konkreten Einsatz des jeweiligen RDF-Datensatzes $a b ;{ }^{13}$ man muss aber wohl die Möglichkeit in Betracht ziehen, dass man mit Linked Data-Einsatz keiner Seite der Alternative Burdick-Brennan entspricht und darin dennoch enormes Potenzial für die digitale Modellierung geistes- und literaturwissenschaftlicher Forschung liegt.

\section{Literaturverzeichnis}

Timothy BRENNAN, The Digital-Humanities Bust. After a decade of investment and hype, what has the field accomplished? Not much, in: The Chronicle of Higher Education, 15.10.2017.

Ann Burdick u. a., Digital_Humanities, Cambridge (Mass.) / London 2012.

Fabio CIOTTI, Digital literary and cultural studies: the state of the art and perspectives, in: Between IV/8 (2014), online unter: http://ojs.unica.it/index.php/between/article/view/1392/1183 (16.04.2019).

Jonathan CULLER, Presupposition and Intertextuality, in: MLN 91/6 (1976), 1380-1396.

Julia KRISTEVA, Bachtin, das Wort, der Dialog und der Roman, in: Dorothee Kimmich / Rolf Günter Renner / Bernd Stiegler, Hg., Texte zur Literaturtheorie der Gegenwart, Stuttgart 2004, 334-348.

Patrick LE BoEUf u. a., Hg., Definition of the CIDOC Conceptual Reference Model. Version 6.2, 2015, 27, online unter: http://www.cidoc-crm.org/sites/default/files/cidoc_crm_version_6.2.pdf (22.4.2019).

12 Ohne an dieser Stelle auf die verschiedenen Alignments von INTRO einzugehen, sei angemerkt, dass CIDOC-CRM, die top-level-Ontologie, an die INTRO anschließt, eine Klasse für diesen genau Zweck bereithält: „E55 Type is the CRM's interface to domain specific ontologies and thesauri. These can be represented in the CRM as subclasses of E55 Type, forming hierarchies of terms [...]." - Patrick LE BOEUF u. a., Hg., Definition of the CIDOC Conceptual Reference Model. Version 6.2, 2015, 27, online unter: http://www.cidoc-crm.org/sites/default/files/cidoc_crm_version_6.2.pdf (22.4.2019).

13 D.h. davon, ob der jeweilige Datensatz bloß zur Speicherung, Dissemination, Visualisierung von Datensätzen als RDF modelliert wird oder auch zum automatisierten Erschließen neuer Daten aus den vorhandenen, zur automatisierten Integration mit anderen Datensätzen etc. 
Bernhard OвERREITHER, INTRO. An Intertextual Relationships Ontology for literary studies, online unter: https:// github.com/BOberreither/INTRO (16.04.2019).

Silvio Peroni / David Shotton, The SPAR Ontologies. Submitted to the 17th International Semantic Web Conference, 2018, online unter: https://w3id.org/spar/article/spar-iswc2018/ (11.05.2018).

Torsten SCHRADE, „Set your data free“ - Vom geisteswissenschaftlichen Datensilo zur vernetzten Wissensbasis mittels Linked Open Data. Siehe Martin PRELL, Digital Humanities in Jena, 23.11.2017 [Tagungsbericht], in: H-Soz-Kult, 27.2.2018, online unter: www.hsozkult.de/conferencereport/id/tagungsberichte-7576 (16.04.2019).

Joseph F. TenNIS, URIs and Intertextuality: Incumbent Philosophical Commitments in the Development of the Semantic Web, in: Knowledge Organization and the Global Information Society: Proceedings of the Eighth International ISKO Conference, Würzburg 2004, 103-108. 


\title{
Forschung öffnen
}

Möglichkeiten, Potentiale und Grenzen von Open Science am Beispiel der offenen DatenBANK „HANDKE: IN ZUNGEN“

\section{Vanessa Hannesschläger}

Österreichische Akademie der Wissenschaften, Austrian Centre for Digital Humanities vanessa.hannesschlaeger@oeaw.ac.at https://www.oeaw.ac.at/acdh/, https://orcid.org/0000-0003-0938-0890

Keywords: Literary Studies, Multilingual Literature, Austrian Literature, Peter Handke, Open Science

\begin{abstract}
This paper analyzes methods, potentials and limits of opening up a research project to other researchers and the public. The project Handke: in Zungen (Handke: in tongues) serves as an example for discussing different aspects of Open Science.
\end{abstract}

\section{Einleitung}

In diesem Beitrag werden die Möglichkeiten und Grenzen der Öffnung eines Forschungsprojekts für andere Forschende, aber auch für die interessierte Öffentlichkeit untersucht. Das Open Science-Projekt Handke: in Zungen dient dabei als Beispiel, anhand dessen verschiedene Aspekte der Öffnung von Wissenschaft diskutiert werden. Neben einer kurzen Vorstellung des Projekts widmet sich der Beitrag den darin angewandten Methoden der Offenen Wissenschaft, aber auch den Anforderungen, die diese mit sich bringen sowie der Frage danach, wie und wozu Offene Wissenschaft konsequent umgesetzt werden kann.

\section{Das Projekt}

Seit den beginnenden 1980er Jahren haben Fremdsprachen in den Bühnentexten des österreichischen Schriftstellers Peter Handke ( $\left.{ }^{*} 1942\right)$ zunehmend an Bedeutung gewonnen. In den frühen, sprachkritischen Stücken der 1960er und 70er Jahre spielen die Sprache, ihre Gemachtheit und die Reflexion darüber die zentrale Rolle - mit einem Umschwung, der sich am „dramatischen Gedicht“ Über die Dörfer (1981) festmachen lässt, werden die Bühnenarbeiten Handkes zunehmend "erzählend“" gewinnen zunehmend an „Handlung“. Mit dieser "Wende“2, mit der auch der Beginn von Handkes Tätigkeit als Übersetzer einhergeht, halten auch die fremden Sprachen Einzug in die Stücke des Autors.

Klaus Kastberger / Katharina Pektor, Vorwort, in: Dies., Hg., Die Arbeit des Zuschauers. Peter Handke und das Theater, Salzburg / Wien 2012, 5.

2 Hans HöLleR, Eine ungewöhnliche Klassik nach 1945. Das Werk Peter Handkes, Berlin 2013. 
Im Rahmen des Dissertationsprojekts „Dramatische Sprachen: Fremdsprachen in den Bühnentexten von Peter Handke" werden sämtliche fremdsprachigen Wörter und Textteile in den beinah 30 Bühnentexten Handkes erhoben und untersucht. Die Leitfragen dabei sind, ob und in welcher Weise bestimmte Sprachen für bestimmte semantische Felder und Themenbereiche eingesetzt werden, welche Sprachen vorherrschen, ob und wie sich die Wichtigkeit einzelner Sprachen im Lauf der Zeit verändert und wie die verschiedenen einfließenden Fremdsprachen miteinander in Beziehung stehen. Für die Analyse dieser Fragen werden die relevanten Textstellen in der relationalen Datenbank Handke: in Zungen gesammelt, wo sie sortier-, durchsuch- und auswertbar gemacht werden. Die Datenbank und das Projekt, in dessen Rahmen sie entsteht, sind der Offenen Wissenschaft verpflichtet und dienen daher als Ausgangspunkt für diesen Beitrag.

\section{Offene Wissenschaft: Prinzipien und Umsetzung}

Die Umsetzung eines offenen Ansatzes in Forschungsprojekten bringt eine Reihe an Themen mit sich, mit denen sich Forschende der traditionellen Geisteswissenschaften nicht vorrangig beschäftigen müssen, die aber in den Digital Humanities ebenfalls von zentraler Bedeutung sind. Zu diesen gehören etwa die Frage nach offener Lizenzierung von Daten, Code und Forschungsergebnissen wie Aufsätzen und Präsentationen, aber auch jene nach deren (langfristiger) Aufbewahrung und Verfügbarmachung, nach adäquater Dokumentation und nach Kommunikation und Vermittlungsarbeit. Das Handke: in Zungen-Projekt eignet sich für eine Diskussion dieser verschiedenen Aspekte von Open Science deshalb in besonderer Weise, weil es unter anderem dank Unterstützung durch Wikimedia Deutschland im Rahmen des Wikimedia-Fellowship-Programms zu Freiem Wissen und Offener Wissenschaft umgesetzt wurde. Aus diesem Grund hat das Projekt neben der eigentlichen Datenbank-Web-App ${ }^{3}$ mehrere online-Präsenzen, die zur Öffnung des Projekts und des darin gesammelten Wissens beitragen.

Das Open Science Network Austria (OANA) definiert die im Folgenden angeführten fünf Kernbereiche Offener Wissenschaft ${ }^{4}$, denen sich auch die verschiedenen digitalen Kanäle, die das Projekt bespielt, zuordnen lassen. Verschiedene Elemente der Öffnung können maßgeblich zum Erfolg eines Forschungsprojekts beitragen, bringen jedoch auch jeweils spezifische Herausforderungen mit sich, die den Fortgang der Arbeit behindern können. Beide Seiten sollen im Folgenden angesprochen werden.

\section{Open Access to Publications}

Der freie Zugang zu wissenschaftlichen Publikationen ist eine mittlerweile etablierte und von den meisten fördergebenden Stellen unterstützte Forderung, die aus rein technischer Perspektive einfach und mit wenig Zeitaufwand umgesetzt werden kann, etwa mittels Veröffentlichung von Aufsätzen und Präsentationsfolien auf zenodo.org ${ }^{5}$. Daher wurde Open Access im vorliegenden Projekt umgesetzt, soweit es rechtlich möglich war. Nicht alle im engeren oder weiteren Sinne aus dem Projekt hervorgegangenen Aufsätze konnten auf diese Weise veröffentlicht werden, weil dem die Rechte von Verlagen entgegenstanden. Da Aufsätze in gedruckten Büchern in der Germanistik nach wie vor die anerkannteste Publikationsmethode sind, konnte dieses Problem aus karrieretechnischen Erwägungen nicht vollständig umgangen werden. Dank des im österreichischen Urheber[*innen]rechtsgesetz fest-

\footnotetext{
3 https://handkeinzungen.acdh.oeaw.ac.at/ (og.04.2019).

4 Open Science Network Austria (OANA): Über Open Science, online unter: https://www.oana.at/ueber-open-science/ (09.04.2019).

5 https://zenodo.org/ (og.04.2019)
} 
gelegten „Zweitverwertungsrecht[s] von Urheber[*inne]n wissenschaftlicher Beiträge“6 (§ 37a) können diese Aufsätze aber nach Ablauf eines Jahres nach Erscheinen online bereitgestellt werden.

\section{Open Research Data}

Neben der Zitatesammlung, die das Herzstück von Handke: in Zungen ausmacht und die mittels API direkt aus der Web-App bezogen werden kann, wurden auch weitere für das Projekt relevante Inhalte als digitale Forschungsdaten aufbereitet: Informationen zu den Uraufführungen aller Bühnentexte Peter Handkes wurden in der Inszenierungsdatenbank theado $k^{7}$ festgehalten und stehen dort der Allgemeinheit zur Weiternutzung zur Verfügung. In einer offenen zotero-Gruppe ${ }^{8}$ wurden die Quellen, aus denen die Zitate in der Datenbank stammen, bibliographisch erfasst. Man kann mit einigem Recht behaupten, dass Open Research Data das Kernelement der Digital Humanities (und ihre Erstellung und Verwendung das wesentliche Unterscheidungsmerkmal zur „traditionellen“ Geisteswissenschaft) sind; ihre Sammlung und Erfassung ist auch die zeitintensivste Aufgabe jedes digitalen Projekts. Der große Aufwand, der mit der Erschaffung von digitalen geisteswissenschaftlichen Forschungsdaten verbunden ist, ist auch das beste Argument für ihre Öffnung, um künftigen Forschenden denselben Aufwand zu ersparen. Die nachhaltige Sicherung der Daten, also ihre Ablage in einem Forschungsdatenrepository, ist laut Datenmanagementplan am Ende des Projekts vorgesehen; zur Nachhaltigkeit der Daten trägt auch ihre offene Lizenzierung bei (CC BY 4.0 International).

\section{Open Evaluation (Open Peer Review, Open Metrics)}

Im Rahmen des Projekts ergab sich bislang keine Möglichkeit, in einem Journal mit Open Peer Review Verfahren zu publizieren. Da solche Journals im relevanten Fach bislang rar bis inexistent sind, hätte die Suche nach solch einem Publikationsmedium einen unverhältnismäßigen Rechercheaufwand bedeutet. (Open) Metrics spielen in der Germanistik bislang quasi keine Rolle.

\section{Citizen Science}

Citizen Science-Methoden im engeren Sinn wurden in diesem Projekt nicht angewandt. Hätte man solche implementieren wollen, hätte das bedeutet, die Web-Applikation mit der Möglichkeit zur Erstellung und Verwaltung von Nutzer*innenprofilen zu versehen. Während der technische Aufwand hierfür zwar vertretbar gewesen wäre, hätte das "Community-Building“, also die Rekrutierung von Citizen Scientists, die Daten erheben und einpflegen, bei einem Nischenthema wie jenem des vorliegenden Projekts ohne zusätzliche finanzielle Mittel zu großen Aufwand bedeutet (und unter Umständen sogar mit größerem Aufwand nicht oder nur bedingt funktioniert). Aus diesem Grund wurde auf die aktive Einbeziehung von forschungsfernen Personen verzichtet und stattdessen nur die Möglichkeit für diese geschaffen, Inhalte und Erkenntnisse des Projekts zu rezipieren. Das geschah digital und analog. Es wurde eine Wikiversity-Seite ${ }^{9}$ (die auch den Datenmanagementplan beinhaltet) sowie ein Twitter-Account ${ }^{10}$ für das Projekt angelegt. Obwohl letzteres Medium eines der effektivsten Mittel zum

$6 \quad$ Bundesgesetz über das Urheberrecht an Werken der Literatur und der Kunst und über verwandte Schutzrechte 2017 (Urheberrechtsgesetz). StF: BGBI. Nr. 111/1936 (StR: 39/Gu. BT: 64/Ge S. 19), online unter: https://www.ris.bka.gv.at/ GeltendeFassung.wxe?Abfrage=Bundesnormen\&Gesetzesnummer=10001848 (09.04.2019).

7 https://theadok.at/ (og.04.2019).

8 https://www.zotero.org/groups/1840645/peter_handke_stage_texts (og.04.2019).

9 https://de.wikiversity.org/wiki/Wikiversity:Fellow-Programm_Freies_Wissen/Einreichungen/Dramatische_Sprachen:_ Fremdsprachen_in_den_B\%C3\%BChnentexten_von_Peter_Handke (o9.04.2019). 
Aufbau und der Pflege einer Interessiertengruppe ist, ist die fortlaufende Betreuung eines Profils in einem sozialen Medium so zeitaufwändig, dass Forschende diese Methode der Öffnung nicht ohne detaillierte und langfristige Planung implementieren sollten. Mit überschaubarerem Zeitaufwand und sehr positiven Rückmeldungen ließen sich dagegen einmalige Publikumsveranstaltungen umsetzen, bei denen das Lai*innenpublikum unmittelbar anwesend war (etwa ein Vortrag im Rahmen des VHS Science-Programms oder Seminare an der Kinderuni Wien).

\section{Open Methodology (Open Source, Open Notebooks)}

Während Open Methodology viele Bereiche umfassen kann, ist Open Source (also die freie Zurverfügungstellung von Programmcode) ihr wahrscheinlich prominentestes Element. Da auch in den Digital Humanities Open Source eine etablierte Praxis ist und keine rechtlichen Argumente dagegensprechen, wurde der Code der Web-App auf GitHub verfügbar gemacht. ${ }^{11}$ Überdies wurde auf GitHub ein zweites Projekt-Repository ${ }^{12}$ angelegt, wo weitere Projekt-Informationen sowie ein Projekt-Logbuch als Open Notebook zur Verfügung gestellt wurden. Ähnlich wie die Betreuung des Twitter-Kanals stellte sich das fortlaufende Befüllung dieses Logbuchs allerdings als unbewältigbar zeitaufwendig heraus.

\section{Conclusio}

Wie hier ausgeführt, haben die zahlreichen Kommunikations- und Distributionskanäle, die von diesem Projekt bespielt werden, jeweils spezifische Vor- und Nachteile, die kurz umrissen wurden. Ebenso haben die spezifischen Voraussetzungen und Rahmenbedingungen jedes Forschungsprojekts (etwa rechtliche Voraussetzungen, aber auch verfügbare Personal- und Zeitressourcen) notwendigerweise Einfluss auf seinen Grad an Öffnung. Vielleicht eine der wichtigsten Erkenntnisse aus dem Öffnungsund Offenheitsanspruch von Handke: in Zungen und seinen digitalen Schwesterkanälen ist, dass der zusätzliche Zeitaufwand, den gewisse Kanäle bedeuten, realistisch eingeschätzt werden muss, um effektiv und erfolgreich zu sein.

Es wird daher hier vorgeschlagen, „Open Science“ nicht als eine strikt definierte Methode mit einem fixen Satz an verpflichtenden Elementen der Öffnung zu verstehen. Vielmehr sollte "Open“ als eine Skala gesehen werden, auf der Projekte, die offene Methoden anwenden wollen, den für sie jeweils angemessenen Platz finden müssen, der von den oben erwähnten Rahmenbedingungen mitbestimmt wird. Grundsätzlich jedoch, so das abschließende Argument dieses Beitrags, sollte sich die geisteswissenschaftliche Forschung - insbesondere die digitale - konsequent auf ihre eigene Öffnung hin orientieren. Dafür sprechen neben praktischen auch ideologische Argumente. ${ }^{13}$ So formulieren es auch Pomerantz und Peek in ihrem Aufsatz Fifty shades of open, in dem die gesellschaftliche Bedeutung von Offener Wissenschaft thematisiert wird: „As the number of open resources of all types increases, the more open resources will be created using them and derived from them, and the more open resources there will be. This snowballing growth of openness is socially beneficial, and, we believe, will make the world a better place."14

11 https://github.com/vanyh/handkeinzungen-app (og.04.2019).

$12 \mathrm{https} / /$ github.com/vanyh/handkeinzungen (09.04.2019).

13 Vgl. dazu Vanessa HANNESSCHLÄGER, Was ist Open Science und zu welchem Ende ist science open?, in: mdw-Magazin 2 (2019), 63f., online unter: https://www.mdw.ac.at/magazin/index.php/2019/o2/25/was-ist-open-science-und-zu-welchemende-ist-science-open/ (09.04.2019).

14 Jeffrey Pomerantz / Robin PEeK: Fifty shades of open, in: First Monday 4 (2016), online unter: http://firstmonday.org/ojs/ index.php/fm/article/view/636o/546o (09.04.2019). 


\section{Literaturverzeichnis}

Bundesgesetz über das Urheberrecht an Werken der Literatur und der Kunst und über verwandte Schutzrechte 2017 (Urheberrechtsgesetz). StF: BGBI. Nr. 111/1936 (StR: 39/Gu. BT: 64/Ge S. 19), online unter: https://www. ris.bka.gv.at/GeltendeFassung.wxe?Abfrage=Bundesnormen\&Gesetzesnummer=10001848 (09.04.2019).

Handke in Zungen App, online unter: https://github.com/vanyh/handkeinzungen-app (07.05.2019).

Handke in Zungen auf Github, online unter: https://github.com/vanyh/handkeinzungen (07.05.2019).

Handke in Zungen auf Twitter, online unter: https://twitter.com/HandkeinZungen (07.05.2019).

Handke in Zungen auf Wikiversity, online unter: https://de.wikiversity.org/wiki/Wikiversity:Fellow-Programm_Freies_Wissen/Einreichungen/Dramatische_Sprachen:_Fremdsprachen_in_den_B\%C3\%BChnentexten_von_ Peter_Handke (07.05.2019).

Handke in Zungen Projekthomepage, online unter: https://handkeinzungen.acdh.oeaw.ac.at/ (07.05.2019).

Vanessa HANNESSCHLÄGER, Was ist Open Science und zu welchem Ende ist science open?, in: mdw-Magazin 2 (2019), 63-64, online unter: https://www.mdw.ac.at/magazin/index.php/2019/02/25/was-ist-open-scienceund-zu-welchem-ende-ist-science-open/ (09.04.2019).

Hans HöLLER, Eine ungewöhnliche Klassik nach 1945. Das Werk Peter Handkes, Berlin 2013.

Klaus Kastberger / Katharina Pektor, Vorwort, in: Dies., Hg., Die Arbeit des Zuschauers. Peter Handke und das Theater, Salzburg / Wien 2012, 5.

Open Science Network Austria (OANA), Über Open Science, online unter: https://www.oana.at/ueber-open-science/ (07.05.2019).

Peter Handke stage texts, online unter: https://www.zotero.org/groups/1840645/peter_handke_stage_texts (07.05.2019).

Jeffrey Pomerantz / Robin Peek: Fifty shades of open, in: First Monday 4 (2016), online unter: http://firstmonday. org/ojs/index.php/fm/article/view/6360/5460 (09.04.2019).

Theadok, https://theadok.at/ (07.05.2019).

Zenodo, https://zenodo.org/ (07.05.2019). 


\title{
Schreiben, wie Godard Filme macht
}

\author{
Präliminarien eines intermedialen Editionsprojekts
}

\section{Wolfgang Straub}

Robert-Musil-Institut für Literaturforschung / Kärntner Literaturarchiv

wolfgang.straub@aau.at

Keywords: Intermediality, Werner Kofler, Hybrid Edition, Edition Philology, Austrian Literature, Digital Edition

\begin{abstract}
Based on the oeuvre of the Austrian writer Werner Kofler (1947-2011) the article focusses on the question how intermediality can be situated in the field of Digital Humanities.
\end{abstract}

Als Roman Jakobson 1959 seinen berühmt gewordenen Vortrag über die Zusammenhänge von Linguistik und Poetik hielt, war für inn und sein Publikum der Gedanke an die „llias“ oder "Odyssee“ als Comic ein lächerlicher. ${ }^{1}$ Heute ist uns dieser Gedanke ein vertrauter, mehr oder weniger die halbe kanonisierte Weltliteratur liegt als Comic oder Graphic Novel vor. Die Ausführungen des Semiotikers und Linguisten Jakobson zeugen davon, wie spätestens um die Mitte des 20. Jahrhunderts das LaokoonParadigma - die in Lessings Schrift geforderte Trennung von Text und Bild - auch in der Literaturwissenschaft seine Gültigkeit einzubüßen beginnt, nachdem zuvor den Avantgarden zu Beginn des 20. Jahrhunderts an medialen Grenzüberschreitungen zu tun gewesen war.

Jakobson spricht von einer „Vergleichbarkeit der verschiedenen Künste“. ${ }^{2}$ Es wird aber bis ins letzte Jahrzehnt des 20. Jahrhunderts dauern, bis die Intermedialität in den Mittelpunkt literaturwissenschaftlichen - und zu Beginn speziell des komparatistischen - Forschungsinteresses rückt; im Rückblick machte man gar einen "intermedial turn“ in der Mitte der 1990er Jahre fest. ${ }^{3}$ Vor dem Hintergrund einer auch im deutschsprachigen Raum ungebrochen umtriebigen Intermedialitätsforschung ${ }^{4}$ will sich mein Beitrag anhand eines anlaufenden literaturwissenschaftlichen Editionsprojekts mit der Frage beschäftigen, ob und wie sich Intermedialität als Forschungsgegenstand im Rahmen der digitalen Geisteswissenschaften positionieren (und fruchtbar machen) lässt.

1 Roman Jakobson, Linguistik und Poetik, in: Roman Jakobsen, Poesie der Grammatik und Grammatik der Poesie. Sämtliche Gedichtanalysen. Hrsg. von Hendrik Birus / Sebastian Donat, Bd. 1: Poetologische Schriften und Analysen zur Lyrik vom Mittelalter bis zur Aufklärung, Berlin / New York 2007, 155-216, hier 157.

2 Ebd.

3 Wolf gibt eine Konferenz im schwedischen Lund 1995, veranstaltet von der Komparatistin Ulla-Britta Lagerroth, als wichtigen Impulsgeber für die internationale Intermedialitätsforschung an. Vgl. Werner WoLF, Towards a Functional Analysis of Intermediality. The Case of Twentieth-Century Musicalized Fiction, in: Erik Hedling / Ulla-Britta Lagerroth, Hg., Cultural Functions of Intermedial Exploration, Amsterdam, New York 2002, 15-34, hier 15. Allg. zum „intermedial turn“ vgl. Christine SCHWANECKE, Filmic Modes in Literatur, in: Gabriele Rippl, Hg., Handbook Intermediality. Literatur - Image - Sound Music, Berlin / Boston 2015, 268-286, hier 272-279.

4 Vgl. u.a.: Uta DEGNER / Norbert Christian WoLf, Hg., Der neue Wettstreit der Künste. Legitimation und Dominanz im Zeichen der Intermedialität, Bielefeld 2010; Evi ZEMANEK, Intermedialität - Interart Studies, in: Evi Zemanek / Alexander Nebrig, Hg., Komparatistik, Berlin 2012, 159-174; Dunja BRÖTz / Beate EdER-JORDAN / Martin FRITZ, Hg., Intermedialität in der Komparatistik. Eine Bestandsaufnahme, Innsbruck 2013; Steven Tötösy de Zepetnek, Hg., Digital Humanities and the Study of Intermediality in Comparative Cultural Studies, Lavayette 2013; Stefan KEPPLER-TASAKI / Wolf Gerhard SCHMIDT, Hg., Zwischen Gattungsdisziplin und Gesamtkunstwerk. Literarische Intermedialität 1815-1848, Berlin 2015. 


\section{Was ist Intermedialität?}

Eine exakte Definition von Intermedialität ist naturgemäß nicht möglich. Das liegt bereits in der Unmöglichkeit begründet, einen eindeutigen Medienbegriff zu postulieren. ,Medium“ kann man, dem konventionellen Verständnis nach und Werner Wolf folgend, als distinkt wahrgenommenes Kommunikationsdispositiv verstehen. ${ }^{5}$ Jörg Robert umreißt, dabei eine erste Definition mitliefernd, den Schritt von der Medien-Definition zur Intermedialität als Bewegung ins Uferlose: „Die Schwierigkeiten mit dem Medienbegriff potenzieren sich im Hinblick auf die Intermedialität. Dies gilt ganz buchstäblich. Intermedialität ist die Bezeichnung für eine Relation zwischen zwei Medien. Beide müssen sich in einer gemeinsamen ,dritten“ Ebene - einer als Universalie angenommenen ,Medialität" - begegnen." 6 Ein Begriff sowohl von Medien als auch von Intermedialität lässt sich nur diskursiv herleiten und ist daher „ebenso vielfältig wie die Diskurse, in denen er verwendet wird. “7

Trotz dieser unumgänglichen begrifflichen Vagheit haben sich nicht wenige Forscher*innen an einer Typologie der Intermedialität versucht. ${ }^{8}$ Die pragmatischste und klarste Darstellung stammt wohl von Irina Rajewsky, die sich dabei mit drei Phänomenen begnügt: Intermedialität im engeren Sinne umfasse einen Medienwechsel (z.B. Literaturverfilmungen); daneben gebe es die Medienkombination, wie sie etwa auf der Opernbühne, am Theater oder in illuminierten Handschriften auftrete; und schließlich seien in der Literatur vor allem die intermedialen Bezüge relevant, etwa bei der klassischen Ekphrasis oder wenn ein Text auf Film Bezug nehme oder wenn man von einer „Musikalisierung der Literatur" spreche. $^{9}$

\section{Was bedeutet Intermedialität im digitalen Zeitalter?}

Für die Frage, wie Intermedialität sich in und mit den „Neuen Medien“ geriere, haben Bolter und Grusin im Jahre 2000 einen in der Folge vieldiskutierten Begriff geprägt: remediation. ${ }^{10}$ Von dieser Begriffsprägung ausgehend verweist der Medienwissenschaftler Jürgen E. Müller darauf, dass man es im Digitalen mit einer Fortsetzung intermedialer Prozesse auf „einem Niveau [zu tun hat], in dem Vorstellungen von Gattungen, Formaten, Funktionen in re-medialisierter oder recycelter Form mit dem Sinnbildungsvermögen des ,Users' spielen.“11

Diese Remedialisierung spielt im derzeit nach wie vor breitesten Feld der digitalen Literaturwissenschaft eine große Rolle: Die digitalen Editionen bilden Bücher ab oder simulieren Bücher, und sie tragen das Literaturarchiv, verschiedenartig aufbereitet und kommentiert, ins Netz. Dabei spielt das WWW die Rolle der von Jörg Robert angesprochenen „dritten Ebene“. Anders verhält es sich, wenn

5 Vgl. Werner WoLF, Intermedialität: Ein weites Feld und eine Herausforderung für die Literaturwissenschaft, in: Herbert Foltinek / Christoph Leitgeb, Hg., Literaturwissenschaft intermedial - interdisziplinär, Wien 2002, 165.

6 Jörg ROBERT, Einführung in die Intermedialität, Darmstadt 2014, 22.

7 Irina O. RAJEWSKY, Intermedialität, light'? Intermediale Bezüge und die ,bloße Thematisierung' des Altermedialen, in: Roger Lüdeke / Erika Greber, Hg., Intermedium Literatur. Beiträge zu einer Medientheorie der Literaturwissenschaft, Göttingen 2004, 27-76, hier 29.

8 Vgl. Jens ScHRÖTER, Discourses and Models of Intermediality, in: Tötösy de Zepetnek, Digital Humanities, 32-42; Werner WoLF, (Inter)mediality and the Study of Literature, in: Ebd., 19-31; Uwe WIRTH, Intermedialität, in: Alexander Roesler / Bernd Stiegler, Hg., Grundbegriffe der Medientheorie, München 2005, 114-121.

9 RAJEWSKY, Intermedialität ,light'?, $37 f$.

10 Vgl. David Jay BolteR / Richard Grusin, Remediation. Understanding New Media, Cambridge/Mass. 200o; zur Kritik an Bolter / Grusin vgl. u.a. Thomas BECKER, Einleitung, in: Ders., Hg., Ästhetische Erfahrung in der Intermedialität, Bielefeld 2011, 7-32, hier $20 f$.

11 Jürgen E. MüLLER, Intermedialität digital. Konzepte, Konfigurationen, Konflikte, in: Andy Bläter u. a., Hg., Intermediale Inszenierungen im Zeitalter der Digitalisierung. Medientheoretische Analysen und ästhetische Konzepte, Bielefeld 2010, 17-39, hier 23. 
das Netz, etwa in der Medienkunst, Teil der intermedialen Interaktion selbst ist. Um beim Beispiel Literatur zu bleiben: Literatur im Netz bringt seit den 1990er Jahren eine eigenständige künstlerische Dimension ein („Netzliteratur“, „multimediale Literatur“), sie dynamisiert und visualisiert Ansätze, die man aus der analogen Literatur kennt, etwa Lautpoesie, konkrete oder visuelle Poesie. Sie macht aus dem Schriftsteller einen Programmierer - oder, im (plakativen) Sinne Kenneth Goldsmiths, einen Textprozessor, der das vorhandene Textmaterial (im Web) nur mehr zu einem weiteren Text organisiert. ${ }^{12}$ Und längst gibt es einen weiteren (Rück-)Schritt der Remedialisierung: In gedruckten Büchern werden digitale Kommunikationsformen abgebildet bzw. simuliert (E-Mail-, Twitter-, Facebook-Romane, gedruckte Blogs, etc.).

\section{3. „Kofler intermedial“}

Das Werk des österreichischen Schriftstellers Werner Kofler (1947-2011) ist prädestiniert wie wenige für eine Analyse intermedialer Phänomene. Kofler ist bekannt für seine satirische Prosa, er war aber auch einer der eifrigsten Hörspielautoren des Landes, er verfasste zwei Theaterstücke und setzte einen Kurzfilm als Regisseur um. Seine Literatur wird als „polyphon“ beschrieben, ihr wird zugesprochen, die „Medialisierung einer selbst schon durch und durch medialisierten Welt“13 zu leisten. Kofler selbst war über den Texttransfer („Mehrfachnutzung“) zwischen Prosa und Radio/Theater/Film hinaus dem Intermedialen zugetan, inm war Musik und Musikalität in seinen Texten von großer Bedeutung. Zudem schweben inm Filme als Vorbilder vor: In einer Vorbemerkung schreibt er von einem „Hörspiel zwischen Godards ,Weekend" und Fellinis ,Orchesterprobe “"'14, in seinen Notizen findet sich der Satz: „Schreiben, wie Godard Filme macht [/] (Es geht.)“15

Nachdem in einem FWF-Forschungsprojekt 2015-2018 die Prosa Koflers in einer kommentierten Hybridedition zugänglich gemacht wurde,$^{16}$ geht nun das Projekt „Kofler intermedial“ mit dem Projektpartner ACDH Graz daran, eine digitale Grundlage zu schaffen für die Analyse von Intermedialität (wobei hier der Text das Ausgangsmedium bleibt). Dabei machen wir uns den Umstand zunutze, dass grundlegende Fragen digitaler Editorik vom jeweiligen Medium unabhängig sind - Roland S. Kamzelak meint etwa, dass „die editorische Arbeit unabhängig von der Quelle gleich“ sei, „sie ist potentiell intermedial." ${ }^{17}$

Wir werden eine Forschungsplattform schaffen, auf der möglichst viele der Radio- und Filmarbeiten sowie Videoaufzeichnungen von Theateraufführungen zur Verfügung stehen. Eine besondere Herausforderung ist dabei der Umstand, dass die Veröffentlichung der Texte Koflers dem Urheberrecht unterliegen. Die Radioarbeiten werden zum Anhören auf der Plattform veröffentlicht, die (aus dem Nachlass generierten) Texte nur in einer analogen Buchversion zugänglich sein. Unsere „Mediensynopse sub auspiciis juris auctoris“ wird also viel mit dem wissenschaftlichen Zitat arbeiten müssen.

12 Vgl. Kenneth GoLDSMITH, Uncreative Writing. Sprachmanagement im digitalen Zeitalter, Berlin 2017.

13 Bernhard FETZ, Stimmen hören. Zu Werner Koflers Triptychon Am Schreibtisch, Hotel Mordschein, Der Hirt auf dem Felsen, in: Herbert J. Wimmer, Hg., Strukturen erzählen. Die Moderne der Texte, Wien 1996, 133-151, hier 141. Werner KofLER, Die vier Jahrezeiten. Hörspiel. Typoskript, Nachlass Kofler, Robert-Musil-Institut für Literaturforschung / Kärntner Literaturarchiv (Klagenfurt), Signatur 11, Mappe W27.

15 Werner KofleR, Notizblock, in: Klaus Amann, Hg., Werner Kofler. Texte und Materialien, Wien 2000, 222-228, hier 224.

16 Vgl. www.wernerkofler.at (15.04.2019).

17 Roland S. KAMZELAK, Mind Mapping (Musik)edition. Versuch einer Zusammenschau, in: Peter Stadler / Joachim Veit, Hg., Digitale Edition zwischen Experiment und Standardisierung. Musik - Text - Codierung, Tübingen 2009 (Beihefte zu editio), 233-236, hier 233. 
Ein Vorteil der Limitation durch das Urheberrecht ist die leichtere Einhaltung der Grenze zwischen den beiden Wissensformationen Archiv und Edition, die Rüdiger Nutt-Kofoth zu Recht eingefordert hat. ${ }^{18}$ Das Archiv (der Nachlass) ist für uns Wissensspeicher für die Erstellung des - im TEI-P5-Standard annotierten und mit Normdaten angereicherten - Stellenkommentars sowie die editorische Entscheidung für die jeweils autorisierte Textversion. Textgenetik ist kein primäres Forschungsinteresse. Bei Kofler sind intramediale Phänomene wie Systemerwähnung (z.B. Ekphrasis, Werberhetorik) oder Systemkontamination („musikalisches Schreiben“), ${ }^{19}$ die am Text untersucht werden können, von großer Bedeutung. Die Innovation von „Kofler intermedial“ wird die Herstellung einer Mediensynopse sein, die es ermöglicht, die Transposition von Textelementen über Mediengrenzen hinweg nachzuvollziehen und damit Formen (und idealerweise auch Funktionen) der Intermedialität zu erschließen. Damit entsteht - mittels ELAN-Annotation und IIIF-Transformation - eine Grundlage für eine intermediale Analyse der Text-Bild- und Text-Akustik-Zusammenhänge.

Digital Humanities sind per se interdisziplinär und intermedial, was aber nicht automatisch bedeutet, dass die Intermedialitätsforschung in den $\mathrm{DH}$ besonders stark vertreten wäre. Sie kann sich zwar „das Internet“ als „Universalmedium“ zunutze machen - ein Medium, das „alle bisherigen analogen Medien auf einer gemeinsamen technischen Basis und aus einem endlichen Vorrat diskreter Zeichen mit Algorithmen simulieren und somit beliebig austauschbar und verknüpfbar machen" ${ }^{20}$ kann. Aber diese „Austauschbarkeit“ wird selten thematisiert; die Verknüpfungen werden allerdings seit dem Aufkommen von Hyperlinks und „Hypermedia“ intensiv zur Vermittlung verwendet, wie sich etwa an dem am MIT entwickelten interaktiven Film „Berliner sehen“ (1995) zeigt. ${ }^{21}$ Kurt Fendt, leitender Entwickler dieses Spracherwerbstools, sieht zwanzig Jahre später in der Online-Intermedialität noch großes Potential. ${ }^{22}$ Wir hoffen, mit unseren literaturwissenschaftlichen Fragestellungen sowohl epistemologisch als auch in der Vermittlung beitragen zu können, die Intermedialitätsforschung in den DH zu stärken. Vielleicht nähern wir uns auf dem Feld der Intermedialität als Literaturwissenschaftler*innen jenem Bild an, das Heimito von Doderer 1957 vom Romancier zeichnete: „Dem Romancier stehen alle Fachwissenschaften zu Gebote, aber er muss möglichst lang Fachwissenschaftler bleiben. ${ }^{23}$

\section{Literaturverzeichnis}

Thomas BECKER, Einleitung, in: Ders., Hg., Ästhetische Erfahrung in der Intermedialität, Bielefeld 2011, 7-32.

David Jay Bolter / Richard Grusin, Remediation. Understanding New Media, Cambridge/Mass. 2000.

Dunja BRÖTZ / Beate EDER-JORDAN / Martin FRITZ, Hg., Intermedialität in der Komparatistik. Eine Bestandsaufnahme, Innsbruck 2013.

Uta DegneR / Norbert Christian Wolf, Hg., Der neue Wettstreit der Künste. Legitimation und Dominanz im Zeichen der Intermedialität, Bielefeld 2010.

18 Rüdiger NUTT-KоFOTH, Edition als Archiv? in: Klaus Kastberger / Stefan Maurer / Christian Neuhuber, Hg., Schauplatz Archiv. Objekt - Narrativ - Performanz, Berlin 2019 (Literatur und Archiv 3), 107-124, hier 117 f.

19 Vgl. Irina RAJEWSKY, Intermedialität, Tübingen / Basel 2002, 83-149.

20 Jörg SCHÄFER, Sprachzeichenprozesse. Überlegungen zur Codierung von Literatur in „alten“ und „neuen“ Medien, in: Jens Schröter / Alexander Böhnke, Hg., Analog/Digital - Opposition oder Kontinuum? Zur Theorie und Geschichte einer Unterscheidung, Bielefeld 2004, 143-168, hier 147.

21 Vgl. http://berlinersehen.mit.edu (15.04.2019).

22 Fendt sieht besonders jenen „Multimedia-Annotierunsgstools“ viel Potential, „die alle über gemeinsame Schnittstellen miteinander kommunizieren [...] und so Archive und Bibliotheken zu so genannten ,layered archives'“ werden lassen können. Markus NeusCHÄFER, Perspektiven der Digital Humanities. Ein Interview mit Kurt Fendt, in: DHd-Blog, 12.10.2015, online unter: https://dhd-blog.org/?p=5735 (15.04.2019).

23 Heimito von DODERER im Gespräch mit Heinz Fischer Karwin [1957]. Nachzuhören auf: Heimito von Doderer 1896-1966. Das Original. 3 CDs. Radio Österreich 1 „Edition Radio Literatur“, 1996, CD 1, Track 1. 
Heimito von DodereR im Gespräch mit Heinz Fischer Karwin [1957]. Nachzuhören auf: Heimito von Doderer 1896-1966. Das Original. 3 CDs. Radio Österreich 1 „Edition Radio Literatur“, 1996, CD 1, Track 1.

Bernhard FETZ, Stimmen hören. Zu Werner Koflers Triptychon Am Schreibtisch, Hotel Mordschein, Der Hirt auf dem Felsen, in: Herbert J. Wimmer, Hg., Strukturen erzählen. Die Moderne der Texte, Wien 1996, 133-151.

Kenneth GoLDSMITH, Uncreative Writing. Sprachmanagement im digitalen Zeitalter, Berlin 2017.

Roman JAKOBSON, Linguistik und Poetik, in: Roman Jakobsen, Poesie der Grammatik und Grammatik der Poesie. Sämtliche Gedichtanalysen. Hrsg. von Hendrik Birus / Sebastian Donat, Bd. 1: Poetologische Schriften und Analysen zur Lyrik vom Mittelalter bis zur Aufklärung, Berlin / New York 2007, 155-216.

Roland S. KamzelaK, Mind Mapping (Musik)edition. Versuch einer Zusammenschau, in: Peter Stadler / Joachim Veit, Hg., Digitale Edition zwischen Experiment und Standardisierung. Musik - Text - Codierung, Tübingen 2009 (Beihefte zu editio), 233-236.

Stefan KEPPLER-TASAKI / Wolf Gerhard SCHMIDT, Hg., Zwischen Gattungsdisziplin und Gesamtkunstwerk. Literarische Intermedialität 1815-1848, Berlin 2015.

Werner KofLER, Die vier Jahrezeiten. Hörspiel. Typoskript, Nachlass Kofler, Robert-Musil-Institut für Literaturforschung / Kärntner Literaturarchiv (Klagenfurt), Signatur 11, Mappe W27.

Werner Kofler, Notizblock, in: Klaus AmAnn, Hg., Werner Kofler. Texte und Materialien, Wien 2000, 222-228.

Jürgen E. MüLLER, Intermedialität digital. Konzepte, Konfigurationen, Konflikte, in: Andy Bläter u. a., Hg., Intermediale Inszenierungen im Zeitalter der Digitalisierung. Medientheoretische Analysen und ästhetische Konzepte, Bielefeld 2010, 17-39.

Markus NeuSCHÄFER, Perspektiven der Digital Humanities. Ein Interview mit Kurt Fendt, in: DHd-Blog, 12.10.2015, online unter: https://dhd-blog.org/?p=5735 (15.04.2019).

Rüdiger NutT-KоғOтH, Edition als Archiv? in: Klaus Kastberger / Stefan Maurer / Christian Neuhuber, Hg., Schauplatz Archiv. Objekt - Narrativ - Performanz, Berlin 2019 (Literatur und Archiv 3), 107-124.

Irina O. RAJEWSKY, Intermedialität, light'? Intermediale Bezüge und die ,bloße Thematisierung' des Altermedialen, in: Roger Lüdeke / Erika Greber, Hg., Intermedium Literatur. Beiträge zu einer Medientheorie der Literaturwissenschaft, Göttingen 2004, 27-76.

Jörg ROBERT, Einführung in die Intermedialität, Darmstadt 2014.

Jörg SCHÄFER, Sprachzeichenprozesse. Überlegungen zur Codierung von Literatur in „alten“ und „neuen“ Medien, in: Jens Schröter / Alexander Böhnke, Hg., Analog/Digital - Opposition oder Kontinuum? Zur Theorie und Geschichte einer Unterscheidung, Bielefeld 2004, 143-168.

Jens SCHRÖTER: Discourses and Models of Intermediality, in: Tötösy de Zepetnek, Digital Humanities, 32-42.

Christine ScHWANECKE, Filmic Modes in Literatur, in: Gabriele Rippl, Hg., Handbook Intermediality. Literatur Image - Sound - Music, Berlin / Boston 2015, 268-286.

Steven TÖTÖSY DE ZEPETNEK, Hg., Digital Humanities and the Study of Intermediality in Comparative Cultural Studies, Lavayette 2013.

Uwe WIRTH, Intermedialität, in: Alexander Roesler / Bernd Stiegler, Hg., Grundbegriffe der Medientheorie, München 2005, 114-121.

Werner WoLF, Intermedialität: Ein weites Feld und eine Herausforderung für die Literaturwissenschaft, in: Herbert Foltinek / Christoph Leitgeb, Hg., Literaturwissenschaft intermedial - interdisziplinär, Wien 2002, 165.

Werner WoLF, Towards a Functional Analysis of Intermediality. The Case of Twentieth-Century Musicalized Fiction, in: Erik Hedling / Ulla-Britta Lagerroth, Hg., Cultural Functions of Intermedial Exploration, Amsterdam / New York 2002, 15-34.

Werner WoLF, (Inter)mediality and the Study of Literature, in: Tötösy de Zepetnek, Digital Humanities, 19-31.

Evi ZeMANEK, Intermedialität - Interart Studies, in: Evi Zemanek / Alexander Nebrig, Hg., Komparatistik, Berlin 2012, 159-174. 


\title{
Annotationen und ihre infrastrukturellen Prämissen
}

\author{
Philipp Hegel [1], Germaine Götzelmann [2], Michael Krewet [1] \& Danah Tonne [2] \\ [1] Freie Universität Berlin \\ [2] Karlsruher Institut für Technologie \\ http://www.sfb-episteme.de/
}

Keywords: Annotations, Digital Infrastructure, Collaborative Research Centre

\begin{abstract}
Since 2016 the Collaborative Research Centre 980 "Episteme in Motion" includes an information infrastructure project which combines humanities with informatics and establishes a central infrastructure for all participating projects. The article describes this infrastructure with its more local or more distributed aspects briefly in two different perspectives: as 'eye of a needle' and as 'groundwork'. The example chosen is the use of digital annotations and a Web Annotation Protocol server used by different projects of the Collaborative Research Centre.
\end{abstract}

Als Jean-François Lyotard 1979 seinen „Bericht über das Wissen“ vorlegte, geschah dies auch als Beschreibung jener Veränderungen, denen das Wissen im digitalen Zeitalter unterliege:

\begin{abstract}
„Il est raisonnable de penser que la multiplication de machines informationnelles affecte et affectera la circulation de connaissances autant que l'a fait le développement de moyens de circulation des hommes d'abord (transports), des sons et des images ensuite (media). Dans cette transformation générale, la nature du savoir ne reste pas intacte. II ne peut passer dans les nouveaux canaux, et devinir opérationnel, que si la connaissance peut être traduite en quantités d'information. [...] Avec l'hégémonie de l'informatique, c'est une certaine logique qui s'impose, et donc un ensemble de prescriptions portant sur les énoncés acceptés comme ,de savoir"“1
\end{abstract}

Diese "Vorherrschaft der Informatik" stellt sich bei Lyotard als ein Flaschenhals dar, durch den das Wissen fließen muss, wenn es in dieser Welt nicht verlorengehen soll. Diskutiert werden sollen hier nicht die Prämissen und Konsequenzen dieser Auffassung. Es sollen vielmehr zwei Sichtweisen mit Blick auf digitale Forschungsinfrastrukturen und anhand eines Beispiels betrachtet werden, die sich eher lose auf Lyotards Stellungnahme beziehen. Zum einen werden digitale Infrastrukturen und Methoden heute oft als Fundament einer Forschung verstanden, die Fragen anders beantwortet und neue Fragen stellt. ${ }^{2}$ Zum anderen bleibt aber doch mit Lyotard zu überlegen, ob und wie sich ein jeweils gegebener Gegenstand oder eine wissenschaftliche Methode in digitalen Verfahren abbilden lässt.

Jean-François LYOTARD, La condition postmoderne. Rapport sur le savoir, Paris 1979, $12 f$.

Wenn im Folgenden von „Infrastrukturen“ gesprochen wird, sind digitale Forschungsinfrastrukturen gemeint. 
Man kann sich fragen, ob diese Objekte und Verfahren durch das Nadelöhr digitaler Infrastrukturen gezogen werden und dabei ,intakt' bleiben können. Diese Punkte betreffen die Frage nach den Bedingungen und Funktionen einer Infrastruktur für die Erzeugung, Tradierung, Wandlung und Nutzung eines bestimmten Wissens und damit auch die Frage nach der Modellierung der Daten und Verfahren.

Digitale Infrastrukturen sollen im Allgemeinen Daten für die und aus der Forschung dauerhaft und zugänglich speichern. ${ }^{3}$ Hinzu kommt die Entwicklung, Einbindung und Pflege von Werkzeugen und Diensten. ${ }^{4}$ Im konkreten Fall umfassen diese beiden Aspekte unter anderem ein Repositorium, einen Server zur Speicherung digitaler Annotationen in einem RDF Triple Store mit SPARQL-Endpoint, ein Werkzeug zur semiautomatischen Layoutanalyse und ein Werkzeug zur digitalen Annotation ihrer Ergebnisse. ${ }^{5}$ Die erforderliche Modellierung der Daten und Annotationen ist auch eine fachliche Entscheidung und kann Auswirkungen auf die fachwissenschaftliche Sicht selbst haben. ${ }^{6}$

Es gibt den Vorschlag, zwischen Forschungsinfrastrukturen lokaler und distribuierter Art zu unterscheiden. Die einen stellten eine „bestimmte Menge von Daten und/oder Werkzeugen zur Verfügung“, die anderen sollen „Standards definieren und Services bereitstellen, welche es erlauben, eine Vernetzung von Daten und Werkzeugen zu ermöglichen und/oder Lösungen für einzelne Fachbereiche oder Problemfelder bereitzustellen“. ${ }^{7}$ Bei diesem Vorschlag wird darauf hingewiesen, dass "die Grenzen hier durchaus fließend verlaufen“. ${ }^{8}$ Eine Infrastruktur innerhalb eines Sonderforschungsbereichs wie die des hier behandelten SFB 980 „Episteme in Bewegung“ kann die Aufgabe haben, Daten aus einzelnen Projekten zu sammeln und Werkzeuge bereitzustellen, aber auch die, die Daten aus Projekten und die dort verwendeten Werkzeuge miteinander zu verbinden. Sie definiert meist keine Standards, kann aber festlegen, welche Standards verwendet werden können und sollten. ${ }^{9}$ Daher scheint es schwierig, sie in dieser Unterscheidung eindeutig zu verorten.

Will man nun die metaphorische Rede vom ,Nadelöhr' oder vom ,Fundament‘ präzisieren, kann dies mit Blick auf die Bedingungen und Funktionen der einzelnen technischen Komponenten geschehen.

3 Heike NEUROTH / Andrea RAPP, Nachhaltigkeit von digitalen Infrastrukturen, in: Bibliothek - Forschung und Praxis 40/2 (2016), 264-270, hier 268, nennen dies „datentechnische Nachhaltigkeit“. Sie beschäftige sich „vordergründig mit dem Nachweis, der Nachnutzung und der persistenten Speicherung von digitalen wissenschaftlichen Sammlungen und Forschungsdaten der Geistes- und Kulturwissenschaften." Schon in diesem Punkt werden auch die gesellschaftlichen und kulturellen Rahmenbedingungen einer Infrastruktur deutlich. Bei der Sammlung des Aristotelismus-Zentrums Berlin, die für das später behandelte Beispiel relevant ist, betrifft dies zum Beispiel die unterschiedlichen Rechte an Mikrofilmen und Digitalisaten von Bibliotheken aus aller Welt.

4 Ebd., 268, bezeichnen Neuroth und Rapp dies als „technische Nachhaltigkeit“ und meinen damit nicht nur die „Stabilisierung der bisherigen Dienste [...], sondern [...] den kompletten Service-Lifecycle.“ Dies lässt sich sowohl auf die „datentechnische" Komponente als auch auf weitere Werkzeuge und Dienste beziehen.

5 Etwas ausführlicher dargestellt in Danah TonNE u.a., Ein Web Annotation Protocol Server zur Untersuchung vormoderner Wissensbestände, in: Patrick Sahle, Hg., Digital Humanities im deutschsprachigen Raum 2019: Multimedial und multimodal, Frankfurt am Main 2019, 285-288, online unter: https://zenodo.org/record/2596o95 (25.03.2019).

6 Die Bedeutung der Modellierung auch für digitale Forschung ist mehrfach hervorgehoben worden. Vgl. zum Beispiel Willard MCCARTY, Modeling, A Study in Words and Meanings, in: Susan Schreibman / Ray Siemens / John Unsworth, Hg., A Companion to Digital Humanities, Oxford 2004, online unter: http://www.digitalhumanities.org/companion (25.03.2019), der zu diesem Verhältnis schreibt: „modeling of something readily turns into modeling for better or more detailed knowledge of it; similarly, the knowledge gained from realizing a model for something feeds or can feed into an improved version." Hervorhebungen im Original.

7 So Gerhard HEYER / Thomas ECKART / Dirk GOLDHAHN, Was sind IT-basierte Forschungsinfrastrukturen für die Geistes- und Sozialwissenschaften und wie können sie genutzt werden?, in: Information. Wissenschaft \& Praxis 66/5-6 (2015), 295303, hier 296.

8 Ebd., 296.

9 Ebd., 296, scheinen Heyer, Eckart und Goldhahn diesen schwächeren Sinn von ,definieren' zumindest unter anderem zu meinen, wenn sie erläutern, dass die Werkzeuge von Forschungsinfrastrukturen „von den definierten Standards und bereitgestellten Services im Sinne einer Integration und Interoperabilität Gebrauch machen“. 
Funktionen können oft variabel, aber nicht beliebig realisiert werden. Hat man ein digitales Werkzeug ausgewählt, sind ,Input', Funktionalität und ,Output' mehr oder weniger strikt festgelegt. ${ }^{10}$ In einer Infrastruktur stehen technische Komponenten zudem nicht für sich allein, sondern reagieren auf die Bedingungen und Möglichkeiten anderer Komponenten und bedingen und ermöglichen zugleich deren Verwendung. ${ }^{11}$ Diese Bedingungen und Funktionen der Komponenten sind auch die Grundlage für ihre Nutzung in verschiedenen Forschungsvorhaben und damit für die Möglichkeit ,distribuierter Infrastrukturen.

Digitale Annotationen bieten sich für ein solche Distribution in einem Verbund an. Erstens ist bei innen die mediale Entkopplung von Anmerkung und annotiertem Objekt zu bemerken. ${ }^{12}$ Infrastrukturelle Stabilität, ${ }^{13}$ wie sie ein Repositorium leisten kann, sowie Möglichkeiten der Referenzierung und Verknüpfung vorhandener Daten ${ }^{14}$ vorausgesetzt, können Annotationen kollaborativ am digitalen Objekt vorgenommen werden. In einem Vorhaben zu etwa 150 griechischsprachigen Handschriften der Überlieferung von De interpretatione eröffnen digitale Annotationen zu Scholien, Diagrammen und Glossen beispielsweise die Möglichkeit, diese Hinzufügungen zu transkribieren und ihre Herkunft zu kennzeichnen, um Wanderungen von einer zur nächsten Handschrift, von einem zu einem anderen Ort nachvollziehbar werden zu lassen. Stellen werden dabei mit projektspezifischen Begriffslisten oder kontrollierten Vokabularen klassifiziert und auf unterschiedlichen Ebenen erschlossen. Die digitalen Annotationen konnten systematisch ausgewertet werden und so in Kombination mit den Handschriftenbeschreibungen exemplarisch einen Textzeugen in einem überlieferungsgeschichtlichen Zweig positionieren, in den man sie, ginge man nur vom aristotelischen Text aus, kaum einordnen würde.

Digitale Annotationen, wie sie das Web Annotation Data Model des W3C-Konsortiums definiert, verhalten sich zweitens relativ neutral zu Medium und Inhalt sowohl der Annotation („body“) als auch des annotierten Gegenstands („target“). Dies erleichtert die Übertragung der Verfahren von einem Projekt wie dem beschriebenen auf ein anderes Forschungsvorhaben etwa zu frühneuzeitlichen Sprachlehrwerken. Die technischen Komponenten werden in diesem Sinne ,distribuiert' eingesetzt, erfordern aber eine spezifische Datenmodellierung und -auswertung, um diese Komponenten ,lokal' in einem konkreten Forschungsprozess und damit fachwissenschaftlich zu verankern.

10 Der ursprünglich psychologisch geprägte Begriff der ,Affordanz' wird in jüngerer Zeit auch zur Beschreibung des Verhältnisses zwischen Akteuren und Artefakten und auch auf digitale Infrastrukturen angewandt. Ulla TscHIDA, Auf der Suche nach dem Artefakt. Zur materiellen Praxis von Infrastruktur-Entwicklung, in: Friederike Elias u.a., Hg., Praxeologie. Beiträge zur interdisziplinären Reichweite praxistheoretischer Ansätze in den Geistes- und Sozialwissenschaften, Berlin 2014, 219-242, hier 221, übernimmt die entsprechende Explikation Andreas Reckwitz und erläutert dies in Hinblick auf ihren Gegenstand: „Die immanenten Strukturen eines technischen Artefakts legen eine Verwendungsweise nahe, determinieren sie aber nicht. Gleichzeitig sind die Handlungsmodi nicht beliebig - die Objekteigenschaften ermöglichen bestimmte Umgangsweisen, andere sind ausgeschlossen." Im Sinne einer wissensgeschichtlichen Wendung könnte ergänzt werden, dass das Wissen der Akteure um diese Bedingungen und Möglichkeiten ausschlaggebend für ihren Umgang mit Artefakten und Infrastrukturen ist.

11 Ebd., 233, erwägt Tschida hierfür ebenfalls die Beschreibung über Affordanzen: „Ohne den Begriff der Affordances überstrapazieren zu wollen, stellt sich die Frage, ob technische Strukturen nicht auch variable, nicht-arbiträre Angebotsstrukturen für andere technische Strukturen aufweisen".

12 Vgl. Harald LoRDick u.a., Digitale Annotationen in der geisteswissenschaftlichen Praxis, in: Bibliothek - Forschung und Praxis 40/2 (2016), 186-199, hier 187: „Nicht zuletzt führt die physische Entkoppelbarkeit der Annotation vom annotierten Objekt zu einer Veränderung des Objekts Annotation selbst.“

13 Vgl. ebd., 196: „Die Verfügbarkeit von Daten spielt eine entscheidende Rolle - zum einem im Hinblick auf annotierbare digitale Gegenstände, zum anderen auf Daten, die als Annotationsinhalte oder Anreicherungen genutzt werden können, z.B. Normdaten."

14 Vgl. ebd., 195: „Für das webbasierte semantische Annotieren ist charakteristisch, [...] Metadaten nicht nur mit eindeutigen Identifikatoren zu versehen, um ihre Interoperabilität und eindeutige Referenzierbarkeit zu gewährleisten, sondern darüber hinaus Verknüpfungen zwischen Ressourcen herzustellen und diese zu typisieren.“ 


\section{Literaturverzeichnis}

Gerhard HEYER / Thomas ECKART / Dirk GOLDHAHN, Was sind IT-basierte Forschungsinfrastrukturen für die Geistes- und Sozialwissenschaften und wie können sie genutzt werden?, in: Information. Wissenschaft \& Praxis 66/5-6 (2015), 295-303.

Harald LoRDICK u.a., Digitale Annotationen in der geisteswissenschaftlichen Praxis, in: Bibliothek - Forschung und Praxis 40/2 (2016), 186-199.

Jean-François LYOTARD, La condition postmoderne. Rapport sur le savoir, Paris 1979.

Willard MCCARTY, Modeling, A Study in Words and Meanings, in: Susan Schreibman / Ray Siemens / John Unsworth, Hg., A Companion to Digital Humanities, Oxford 2004, online unter: http://www.digitalhumanities.org/ companion (25.03.2019).

Heike NEUROTH / Andrea RAPP, Nachhaltigkeit von digitalen Infrastrukturen, in: Bibliothek - Forschung und Praxis 40/2 (2016), 264-270.

Danah TONNE U.a., Ein Web Annotation Protocol Server zur Untersuchung vormoderner Wissensbestände, in: Patrick Sahle, Hg., Digital Humanities im deutschsprachigen Raum 2019: Multimedial und multimodal, Frankfurt am Main 2019, 285-288, online unter: https://zenodo.org/record/2596095 (25.03.2019).

Ulla TschIDA, Auf der Suche nach dem Artefakt. Zur materiellen Praxis von Infrastruktur-Entwicklung, in: Friederike Elias u.a., Hg., Praxeologie. Beiträge zur interdisziplinären Reichweite praxistheoretischer Ansätze in den Geistes- und Sozialwissenschaften, Berlin 2014, 219-242. 


\title{
Kulinarische Transfers aus anderen Regionen und Kulturräumen: der Niederschlag in westdeutschen und österreichischen Kochbüchern 1950-1980
}

\author{
Ein Werkstattbericht Über einen „Mixed methods“-Ansatz aus kulturwissenschaftlichem \\ Close Reading und digitalen Methoden
}

Christoph Bläsi \& Edith Blaschitz

Keywords: Cookbooks, Culinary Transfers, Mixed Methods

\begin{abstract}
This project investigates how culinary transfers of 'foreign' food are reflected in Austrian and German cookbooks from 1950 to 1980. For this, methods from Digital Humanities are combined with cultural studies approaches.
\end{abstract}

\section{Einleitung}

Im dargestellten Forschungsprojekt wird untersucht, wie sich kulinarische Transfers aus anderen Regionen und Kulturräumen in westdeutschen und österreichischen Kochbüchern der Jahre 1950-1980 widerspiegeln. Erste Ergebnisse des interdisziplinären Projektes, in dem Daten sowohl mit Methoden der Digital Humanities als auch mit hermeneutisch-kulturwissenschaftlichen Herangehensweisen analysiert werden, liegen nun vor.

\section{Das Kochbuch als Medium und Forschungsgegenstand}

Obgleich das Kochbuch ein am Buchmarkt anhaltend relevantes Genre ist, das 2017 in Deutschland mit 25,6 \% den höchsten Anteil im Ratgebersegment einnahm ${ }^{1}$, findet es als Untersuchungsgegenstand in der Buchwissenschaft unangemessen wenig Beachtung. Die Buchwissenschaft sieht sich hier grundsätzlich zumindest auch zuständig, insoweit sie zwar zunächst Bücher aus Produktions-, Distributions- und Rezeptionsperspektive, insbesondere im Hinblick auf Aspekte von Materialität und Medialität der Buchkommunikation, beforscht, sich als dann gewissermaßen Disziplin vernachlässigter Gegenstände aber nicht selten auch inhaltlicher Aspekte non-fiktionaler Buchgenres annimmt; ein Beispiel dafür zitieren wir unten.

In den kulturwissenschaftlich orientierten Medien- und den Literaturwissenschaften ${ }^{2}$ wird das vorwiegend non-fiktionale Kochbuch ebenso wenig wahrgenommen. ${ }^{3}$ Kochbücher werden hingegen in

1 Börsenverein des Deutschen Buchhandels, Buch und Buchhandel in Zahlen 2018. Zahlen, Fakten und Analysen zur wirtschaftlichen Entwicklung, Frankfurt am Main 2018, 18.

2 Jens RuchatZ, Kochbücher und andere Kochmedien, Vortrag 19.11.2018, Workshop „Kulturelle Aneignungs- und Bewahrungsprozesse und deren mediale Resonanzen am Beispiel von ,Kochwissen““, Johannes-Gutenberg-Universität Mainz.

3 Siehe etwa Hermann CöLFTEN, Vom Kochrezept zur Kochanleitung: sprachliche und mediale Aspekte einer verständlichen Vermittlung von Kochkenntnissen, in: Unikate: Berichte aus Forschung und Lehre 30 (2007), 84-93; Jonatan LEER u. a., 
den geisteswissenschaftlich orientierten Food Studies (Ethnologie, Geschichtswissenschaften etc.) herangezogen, wenn Entstehung und identitätsschaffender bzw. -wahrender Charakter von Regionaloder Nationalküchen untersucht werden ${ }^{4}$. Die Digital Humanities nehmen sich Kochbüchern an, fokussieren dabei im Wesentlichen aber die Produktion annotierter digitaler Kochbucheditionen. ${ }^{5}$

War das Kochbuch, das vor allem ab dem späten 19. Jahrhundert eine nennenswerte Verbreitung fand, zunächst ein vorwiegend der Kochpraxis dienendes Textbuch, das mit Rezepten Handlungsanleitungen zur Zubereitung von Gerichten bot, haben sich ab den 1920er-Jahren mehrere der genannten Bestimmungsgrößen sukzessive aufgeweicht: Ein modernes Kochbuch ist, so kann man z. B. mit Bateman et al. ${ }^{6}$ sagen, heute typischerweise ein komplexer multimodaler Text, bei dem mit sprachlichen Elementen (immer mehr mit narrativen Textpassagen, oft zur Kontextualisierung des Rezeptes), auch mit Bildern (aus der „Food“-, aber ebenso aus der so genannten „Mood“-Fotografie) sowie in hohem Maße mit den Möglichkeiten des Layouts (Spalten, Weißräume, Boxen etc.) gearbeitet wird. Zu den konstitutiven Bestandteilen auf der inhaltlichen Ebene gehören Rezepte, die im Kern aus Zutatenliste und Zubereitungsanweisungen bestehen, aber eben, wie erwähnt, auch Rahmentexte (z. B. zu Grundlagen des Kochens, zu Klassen von Gerichten, zum Kontext wie zu Regionen oder Kulturräumen etc.) sowie Bilder und ggf. andere Illustrationen.

Zusätzlich zu den genannten inhaltlichen und formalen Entwicklungen des Genres - und z.T. direkt mit diesem korrespondierend - fand auch eine Funktionserweiterung des Kochbuchs statt: Es wird nicht mehr notwendigerweise nur als Ratgeber für die Zubereitung von Gerichten verwendet, sondern kann z. B. auch als Ausdruck des eigenen Habitus gesammelt und präsentiert werden. Außerdem kann es als „Phantasiematerial“7 dienen, um sich in eine narrative Welt ,transportieren“ zu lassen, wie man das sonst nur als Nutzungsoption bei fiktionalen Texten kennt ${ }^{8}$.

\section{Regionale, nationale und ,fremde` Küche im Kochbuch}

Kochbücher sind immer von den jeweiligen gesellschaftlichen, politischen und sozioökonomischen Rahmenbedingungen beeinflusst. Mit einer massenhaften Verbreitung von Kochbüchern im 19. Jahrhundert erfolgte ein Vergesellschaftungsprozess von ,Kochwissen': Das Wissen über die Zubereitung von Speisen und das Repertoire an Gerichten wird nicht mehr nur individuell weitergegeben (also z. B. von der Mutter an die Tochter), sondern auch über das Medium Buch. Mit der Mediatisierung findet ein Normierungsprozess statt. Kochbücher sind somit nicht nur mediale Repräsentanten

Food and media: practices, distinctions and heterotopiaLondon, NY 2016.

4 Siehe exemplarisch: Kirsten Schlegel-MatthiES, Regionale Speisen in deutschen Kochbüchern des 19. und 20. Jahrhunderts, in: Hans Jürgen Teuteberg u. a., Hg., Essen und kulturelle Identität. Europäische Perspektiven, Berlin 1997, 212-227; Nicola HumBLE, Culinary Pleasures. A History of Britain through Cookbooks, London 2005; Ronda L. BRULOTTE / Michael A. Dı GIOVINE, Edible Identities: Food as Cultural Heritage, Farnham 2014; Maren MöHRING, Fremdes Essen. Die Geschichte der ausländischen Gastronomie in der Bundesrepublik Deutschland, München 2012; Regina FRISCH, Biografie eines Kochbuchs: das Bayerische Kochbuch erzählt Kulturgeschichte, Regensburg 2016; Peter NACCARATO u. a., Representing Italy through Food, London 2018.

5 Siehe exemplarisch Helmut W. KLUG / Karin KRANICH, Das Edieren von handschriftlichen Kochrezepttexten am Weg ins digitale Zeitalter - Zur Neuedition des Tegernseer Wirtschaftsbuches, in: Thomas Bein, Hg., Vom Nutzen der Editionen. Zur Bedeutung moderner Editorik für die Erforschung von Literatur- und Kulturgeschichte, Berlin / Boston 2015, $121-137$.

6 John Bateman / Janina Wildfeuer / Tuomo HiIPPALA, Multimodality. Foundations, Research and Analysis. A ProblemOriented Introduction, Berlin 2017.

7 Klaus-Ulrich PECH (1998), zit. in: Anke Vogel / David Oels, Wie und warum man Kochbücher verwendet. Eine empirische Studie, in: David Oels / Michael Schikowski, Hg., Non-Fiktion - Ratgeber, Hannover 2012, 127-142, hier 138.

8 Melanie C. GreEN u. a., Understanding Media Enjoyment: The Role of Transportation Into Narrative Worlds, in: Communication Theory 14/4 (2004), 311-327. 
gesellschaftlicher Prozesse, sondern sie konstruieren diese mit, schreiben sie im wahrsten Sinne des Wortes fest. So wurden im Prozess des „Nation Buildings“ im 19. Jahrhundert bestimmte Speisen zum Kanon einer Nationalküche in Kochbüchern zusammengefasst. Diese trägt zur Konstituierung einer eigenen nationalen Identität bei - und damit auch zur Abgrenzung nach außen (also der Konstruktion des ,Eigenen“ im Gegensatz zum ,Fremden').

\section{Untersuchung des Niederschlags kulinarischer Transfers aus anderen Regionen und Kulturräumen in westdeutschen und österreichischen Kochbücher 1950-1980}

Das Projekt der Donau-Universität Krems und der Johannes-Gutenberg-Universität Mainz untersucht den Niederschlag von kulinarischen Transfers aus Regionen und Kulturräumen, die sich im Untersuchungszeitraum nicht innerhalb des westdeutschen oder des österreichischen Staatsgebietes befanden, anhand eines ausgewählten Bestandes an deutschen und österreichischen Kochbüchern aus dem Zeitraum von 1950 bis 1980. Unterstützt wird das Projekt - vor allem wegen seines Digital Humanities-Anteils - von mainzed, dem Mainzer Zentrum für Digitalität in den Geistes- und Kulturwissenschaften.

Der gewählte Zeitraum 1950 bis 1980 wird in der Bundesrepublik Deutschland und in Österreich neben anderen Transformationen mit einem massiven ,Internationalisierungsschub“ verbunden. Bei der Untersuchung der - Vorkriegstraditionen geschuldeten - kontinuierlichen Verwendung bzw. Neuaufnahme von Zutaten, Zubereitungsweisen und ganzen Rezepten aus anderen Regionen und Kulturräumen müssen diese dafür nicht nur als solche identifiziert werden, sonders es stellen sich auch Fragen wie die, ob erkennbar wird, mit welchen Konnotationen, Bewertungen etc. durch die Autor*innen bzw. Herausgeber*innen diese Aufnahme erfolgt ist und ob sich nachvollziehbar Vermutungen anstellen lassen, welche Vorstellungen und Zielgruppenmodellierungen der Grund für die Aufnahme gewesen sein könnten.

Das hier darzustellende Forschungsprojekt betrachtet die Aufnahme von Zutaten, Zubereitungsweisen und Rezepten aus anderen Regionen und Kulturräumen in Kochbücher als eine Folge gesellschaftlicher Kontinuitäten als auch von Transformationen verschiedener Art. Kochbücher ,framen' dabei oft explizit, zumindest aber implizit, die Aufnahme von bestimmten Rezepten (aus welchen Gründen diese auch immer redaktionell erfolgt ist). Der Begriff des ,Fremden“ fasst - sozusagen als ein Begriff unserer ,Werkstattsprache - zwar immer wieder sehr plastisch, wie das, um das es in vielen Fällen geht, vortheoretisch gesehen werden kann - an Beispielen wie dem ungarischen Gulasch, das nach dem Ende der k.u.k.-Monarchie für Österreich zwar staatspolitisch entfremdet, für die österreichische Standardküche als Gericht aber weiterhin als ,Eigenes' betrachtet wurde, sieht man aber, dass das Gegensatzpaar aus ,Eigenem، und ,Fremdem' als zentrale Dichotomie zu viel theoretischen Ballast mitbringt und überdies auch nicht den ganzen Raum der uns interessierenden Phänomene aufspannt. Wir haben deshalb die Arbeit am Thema zwar mit einem Pilotprojekt mit den das ,Fremde' anspielenden Begriffen von Aneignung und Bewahrung im Titel (,Kulturelle Aneignungs- und Bewahrungsprozesse und deren mediale Resonanzen am Beispiel von ,Kochwissen“") gestartet ${ }^{9}$, sind im Verlauf der Forschungsarbeit aber zu der Einsicht gekommen, dass es uns präziser - und so fassen wir das ja auch in dieser Darstellung - um den Niederschlag von kulinarischen Transferprozessen in Kochbüchern geht.

9 Pilotprojektdauer: November 2017-November 2018, gefördert von: Inneruniversitäre Forschungsförderung (Stufe I), Johannes-Gutenberg-Universität Mainz. 


\subsection{Unser grundsätzliches Vorgehen}

Schon weil mehrere der oben angedeuteten bestehenden Forschungsstränge - vor allem natürlich der zur kulturellen Rolle und Funktion von Nahrungszubereitung und Essen und der zum Medium Kochbuch - für die gegebene Fragestellung Aufmerksamkeit verdienen, bietet sich im Kern eine Forschungszusammenarbeit aus einer Kulturwissenschaftlerin und einem Buchwissenschaftler, wegen des methodischen Ansatzes unterstützt von Computerphilologen, unmittelbar an. Die sich daraus ergebende grundsätzliche Arbeitsweise kann folgendermaßen beschrieben werden: Aufgrund der kulturwissenschaftlich-philologischen Betrachtung von Kochbüchern gewonnene Fragen und Arbeitshypothesen werden mit computerphilologischen Methoden überprüft, die Ergebnisse davon hermeneutisch - also vor allem mit Bezug auf relevante Kontexte - ausgewertet und aus diesen - in einem nächsten Durchgang des zirkulären Vorgehens - neue Fragen und Arbeitshypothesen entwickelt. Die Entscheidung für einen „Mixed Methods“-Ansatz beruht nicht zuletzt auf der Tatsache, dass z. B. der oben erwähnte Grund für die Aufnahme eines Rezeptes meist nicht oder nicht explizit im untersuchten Kochbuch selbst ablesbar ist, sondern dass Vermutungen im Hinblick auf den Aufnahmegrund einer Kontextualisierung mit anderen Texten und auch gesellschaftlichen etc. Gegebenheiten bedürfen.

\subsection{Wo wir stehen}

Als Grundlage für unsere Pilotarbeit haben wir zunächst zwei Standard-Kochbücher - das „Franz Ruhm Kochbuch" und das „Dr. Oetker Schulkochbuch"10 von einem Dienstleister scannen, der optischen Zeichenerkennung unterziehen und dann nach dem TEI-Tite-Standard vorstrukturieren lassen. Parallel dazu haben wir eine größere Anzahl von Kochbüchern aus dem Betrachtungszeitraum untersucht und Arbeitshypothesen zunächst v.a. dazu entwickelt, wie sich kulinarische Transfers in Kochbüchern niederschlagen können bzw. welche textlichen (und bildlichen!) Gegebenheiten als Marker-Kandidaten für ,Transfer-Rezepte' herangezogen werden können. Zu den auf diese Weise identifizierten Markern gehören vor allem in spezifizierten Referenzkochbüchern (aus der Zeit vor 1950) noch nicht oder noch nicht in der Häufigkeit vorkommende Zutaten (z. B. Olivenöl), Bezeichnungen, in denen Namen von Orten aus dem Quellgebiet des Transfers vorkommen (z. B. „bosnische Suppe“) oder sonstige sprachliche Elemente aus den Sprachen des Quellgebietes (z. B. „à la mère“). Relevant sind aber auch Rahmentexte, die Transfers implizit oder explizit identifizieren oder sogar bewerten, indem sie z. B. auf Migrations- oder Urlaubserfahrungen anspielen.

Einfachere aus diesen kulturwissenschaftlich erarbeiteten Markern (z. B. Städte- und Ländernamen, Zutaten wie Käsesorten) haben wir dann in einem nächsten Schritt zur Grundlage genommen, um in den digitalisierten Kochbüchern automatisiert zu überprüfen, ob die Operationalisierung hermeneutisch plausible Ergebnisse bringt. Die Nutzung von autoritativen Listen von Städten und Ländern sowie von Lebensmittelgruppen, z. B. Käsesorten, hat hier in der Tat unmittelbar Ergebnisse gebracht - also Kandidaten für ,Transfer-Rezepte‘ identifiziert, einige von diesen durchaus unerwartet.

10 Franz Ruнm, Das Franz Ruhm Kochbuch. Das Standardwerk der Wiener und österreichischen Küche, Wien 1980; Dr. Oetker Schulkochbuch, Bielefeld 1952. 


\section{Was wir gelernt haben und was jetzt ansteht}

Wir haben gesehen, dass die ursprünglich als ein nächster Schritt vorgesehene Tiefenstrukturierung der Kochbücher (z. B. die Auszeichnung von Mengen und Zutaten) in TEl (lite oder ,proper') weniger prioritär sein dürfte als angenommen - viele der ins Auge gefassten computerphilologischen Methoden (z. B. für die erwähnten Bewertungen: Emotion Analysis) erfordern nicht zwingend strukturierte Texte. Auf der anderen Seite wurde sehr klar, dass die Beobachtung der Entwicklung über die gesamte Untersuchungszeit konstitutiv wichtig ist, da es eine Frequenzentwicklung von relativen Vorkommen von Namen und Begriffen gibt, die mit der Unterscheidung „Vorkommen / Nicht-Vorkommen“ nicht angemessen erfasst werden kann. Dafür ist die Vergrößerung des Korpus an digitalisierten Kochbüchern essentiell. Jenseits des Begriffs des ,Fremden', den wir als zu operationalisierendes Konstrukt hinter uns gelassen haben, müssen wir die Liste der Marker theoriebasiert erweitern und diese um weitere Operationalisierungen des Niederschlags kulinarischer Transfers aus anderen Regionen und Kulturräumen ergänzen. Auch scheint über den Einbezug von Referenzkochbüchern aus der Zeit vor 1950 eine Erweiterung um weiteres sprachliches ,Referenz-Material' wichtig; zu diesem gehören etwa Werke aus der Kochausbildung sowie Texte, die Erfahrungen und Bewertungen von Migration oder von Urlaub, aber z. B. auch von gesunder Lebensweise - ein weiterer möglicher Aufnahmegrund von Rezepten anderer Provenienz - erfassen. Neben der Verfeinerung des Instrumentariums zur Analyse von Textuellem gilt es im nächsten Schritt zumindest auch leichtgewichtigere Methoden der Bildanalyse einzubeziehen (wir sehen Kochbücher im Untersuchungszeitraum weiterhin als primär textlich konstituierte Artefakte), da sich ,Fremdheit', auch das ein Ergebnis der ersten Phase, in einigen Fällen erst über bildliches Material erschließt.

\section{Literaturverzeichnis}

John BAteman / Janina WiLdfeuer / Tuomo HiIPPALA, Multimodality. Foundations, Research and Analysis - A Problem-Oriented Introduction, Berlin 2017.

Börsenverein des Deutschen Buchhandels, Buch und Buchhandel in Zahlen 2018. Zahlen, Fakten und Analysen zur wirtschaftlichen Entwicklung, Frankfurt am Main 2018.

Ronda L. BRULOtTE / Michael A. DI GIovine, Edible Identities: Food as Cultural Heritage, Farnham 2014.

Hermann CöLFTEN, Vom Kochrezept zur Kochanleitung: sprachliche und mediale Aspekte einer verständlichen Vermittlung von Kochkenntnissen, in: Unikate: Berichte aus Forschung und Lehre 30 (2007), 84-93.

Regina FRISCH, Biografie eines Kochbuchs: das Bayerische Kochbuch erzählt Kulturgeschichte, Regensburg 2016.

Melanie C. GReEN u. a., Understanding Media Enjoyment: The Role of Transportation Into Narrative Worlds, in: Communication Theory 14/4 (2004), 311-327.

Nicola Humble, Culinary Pleasures. A History of Britain through Cookbooks, London 2005.

Helmut W. KLUG / Karin KRANICH, Das Edieren von handschriftlichen Kochrezepttexten am Weg ins digitale Zeitalter - Zur Neuedition des Tegernseer Wirtschaftsbuches, in: Thomas Bein, Hg., Vom Nutzen der Editionen. Zur Bedeutung moderner Editorik für die Erforschung von Literatur- und Kulturgeschichte, Berlin / Boston 2015, 121-137.

Jonatan LEER u. a., Food and media: practices, distinctions and heterotopia, London / NY 2016.

Maren MöHRING, Fremdes Essen. Die Geschichte der ausländischen Gastronomie in der Bundesrepublik Deutschland, München 2012. 
Peter NACCARATO u. a., Representing Italy through Food, London 2018.

Dr. Oetker Schulkochbuch, Bielefeld 1952.

Klaus-Ulrich PECH (1998), zit. in: Anke Vogel / David Oels, Wie und warum man Kochbücher verwendet. Eine empirische Studie, in: David Oels / Michael Schikowski, Hg., Non-Fiktion - Ratgeber, Hannover 2012, 127-142.

Jens RUCHATZ, Kochbücher und andere Kochmedien, Vortrag 19.11.2018, Workshop „Kulturelle Aneignungs- und Bewahrungsprozesse und deren mediale Resonanzen am Beispiel von ,Kochwissen“, Johannes-Gutenberg-Universität Mainz.

Franz Ruнm, Das Franz Ruhm Kochbuch. Das Standardwerk der Wiener und österreichischen Küche, Wien 1980.

Kirsten ScHLEgEL-MATTHIES, Regionale Speisen in deutschen Kochbüchern des 19. und 20. Jahrhunderts, in: Hans Jürgen Teuteberg u. a., Hg., Essen und kulturelle Identität. Europäische Perspektiven, Berlin 1997, 212-227. 


\section{Keyword Index}

A

Academic Fields 77

Aichinger, llse 37

Analogy 131

Annotations 153

App 19

Archaeology 73

Astronomical Knowledge 32

Audience-Oriented Digitalization 120

Augmented Reality 19

Austrian Literature 37, 126, 143, 148

Authority Files 70

Auxiliary Data 86

\section{B}

Baroque Era 57

Biblatex 37

Bibliography 37

Bibtex 37

\section{C}

Cartographic Communication 24

Cartographic Visualization 24

Cinema History 82

Citizen Science 82, 94

Collaboration 70

Collaborative Research Centre 153

Color Information 57

Computational Literary Studies 98

Content Marketing 120

Controlled Vocabulary 131

Cookbooks 157

Crowdsourcing 94

Culinary Transfers 157

Cultural Tourism 19

\section{D}

Digital Archaeology 13, 77

Digital as Primary Source 13

Digital Ecosystem 13

Digital Edition 43, 86, 148

Digital Infrastructure 153

Digital Preservation 70

Digital Sources 13

\section{E}

Early Modern Prints 65

EbnerOnline 49

Edition Philology 148

E-journal 115

Embedded Librarianship 70

Ephesos 73

\section{$\mathbf{F}$ \\ Feature Analysis 57}

G

Genre Classification 65

Guide 19

H

Handke, Peter 143

HGIS 24

Historical Administrative Sources 86

Historical Landscape 24

historioPLUS 115

History 94

History of Everyday Life in the GDR 82

Hybrid Edition 86, 148

\author{
I \\ Identity 131 \\ Image 57 \\ Interdisciplinarity 43 \\ Intermediality 148 \\ Internet 13 \\ Intertextuality 137 \\ IT-Infrastructure 49
}

$\mathbf{K}$

Kofler, Werner 148 
L

Library Data 70

Linguistics 94

Linked Open Data 73, 86, 137

Literary Annotation 98

Literary Network Analysis 98

Literary Studies 137, 143

Long-Term Archiving 49

\section{M}

Metadata Enrichment 65

MHDBDB 49

Mixed Methods 157

Mozart, Wolfgang Amadé 43

Multilingual Literature 143

Music Encoding Initiative (MEI) 43

\section{N}

Nachlass 126

Navigation 19

\section{$\mathbf{0}$}

OCR 94

OCR-Post-Correction 65

Ontology 126, 131, 137

Open Access 73, 115

Open Science 143

\section{$\mathbf{P}$}

Paintings 57

Platform 19

Public History 82
$\mathbf{R}$

RDF 131

Repository 49

Resource Discovery 126

Roman Imperial Period 32

Round Earth 32

\section{$\mathbf{S}$}

Science Marketing 120

Semantic Web 126, 131, 137

Spatial Uncertainty 24

Stefan Zweig digital 49, 126

\section{$\mathbf{T}$}

Taxonomy 77

Text Encoding Initiative (TEI) 43, 86, 126

Theory 77

Transcription 94

Travelogues 65

$\mathbf{U}$

Urban Gazetteer 73

V

Virtual Research Infrastructure 82

W

Wikidata 37

Worldview 32

$\mathbf{Z}$

Zweig, Stefan 49 